\title{
Mammalian spermiogenesis and the formation of the sperm head to tail coupling apparatus
}

\author{
Dissertation \\ for the award of the degree \\ "Doctor rerum naturalium" (Dr. rer. nat.) \\ of the Georg-August-Universität Göttingen
}

within the doctoral program Biology

of the Georg-August University School of Science (GAUSS)

submitted by

Constanza Tapia Contreras

from Victoria, Chile

Göttingen, 2021 


\section{Thesis committee}

Supervisor: Prof. Dr. Sigrid Hoyer-Fender

Department of Developmental Biology - Johann-Friedrich-Blumenbach-Institute of Zoology and Anthropology - Georg-August-University of Göttingen

Co-supervisor: Prof. Dr. Rüdiger Behr

Platform Degenerative Diseases

German Primate Center Göttingen

\section{Members of the Examination Board}

First reviewer: Prof. Dr. Sigrid Hoyer-Fender

Department of Developmental Biology GZMB - Johann-Friedrich-Blumenbach-Institute of Zoology and Anthropology - Georg-August-University of Göttingen

Second reviewer: Prof. Dr. Rüdiger Behr

Platform Degenerative Diseases

German Primate Center Göttingen

Prof. Dr. Ernst Wimmer

Department of Developmental Biology GZMB - Johann-Friedrich-Blumenbach-Institute of Zoology and Anthropology - Georg-August-University of Göttingen

Dr. Melina Schuh

Department of Meiosis - Max-Planck-Institute for Biophysical Chemistry

Prof. Dr. Hubertus Jarry

Department of Research Animal Welfare Officer - University Medical Center Göttingen

Prof. Dr. Peter Burfeind

Institute of Human Genetics - University Medical Center Göttingen

Date of the oral examination: 18. 05. 2021 


\section{Declaration}

I hereby declare that the Dissertation "Mammalian spermiogenesis and the formation of the sperm head to tail coupling apparatus" was a product of my experimental doctoral project carried out in the Department of Developmental Biology, Georg-August University Göttingen. Further, I confirm that this thesis has been prepared independently and with no other sources and aids than quoted.

Constanza Tapia

Publications that are part of this thesis:

Tapia Contreras, C. \& Hoyer-Fender, S. (2020). The WD40-protein CFAP52/WDR16 is a centrosome/basal body protein and localizes to the manchette and the flagellum in male germ cells. Scientific Reports 10 (1), 14240. https://doi.org/10.1038/s41598-020-71120-9

Tapia Contreras, C. \& Hoyer-Fender, S. (2019). CCDC42 localizes to manchette, HTCA and tail and interacts with ODF1 and ODF2 in the formation of the male germ cell cytoskeleton. Frontiers in Cell and Developmental Biology, 7, 151. https://doi.org/10.3389/fcell.2019.00151 


\section{Dedication}

To my Family and Lukas 


\section{Acknowledgment}

First and foremost, I would like to express my sincere gratitude to my advisor and mentor, Prof. Dr. Sigrid Hoyer-Fender. I am very grateful to her for giving me the opportunity and allowing me to be her doctoral student. I thank her so much for the valuable scientific training, guidance, encouragement, and assistance throughout my studies, especially for her patience, unconditional support and for being always willing to talk.

Besides my advisor, I would like to thank Prof. Dr. Rüdiger Behr for accepting to be part of my thesis committee, for his help and scientific support. I want to thank Prof. Dr. Ernst Wimmer for letting me be part of the Department of Developmental Biology and providing the scientific environment to carry out this investigation. I am also grateful to Dr. Melina Schuh, Prof. Dr. Ernst Wimmer, Prof. Dr. Hubertus Jarry, and Prof. Dr. Peter Burfeind for taking their time and accepting to be part of my extended committee.

I would like to thank all the people from the Department of Developmental Biology whose assistance was valuable in the fulfillment of this project, including technicians and secretaries. Thanks to Atika, Amel, Diana, Zahra, and Cera for your support and for providing a nice and friendly working atmosphere in our lab. Thanks to Gloribel and Elena for your patience, understanding, advice, and pleasant conversations.

I gratefully acknowledge the scholarship provided by the Chilean National Commission for Scientific and Technological Research (CONICYT), the German Academic Exchange Service (DAAD), and the Department of Developmental Biology, GZMB, Georg-August-Universität Göttingen to undertake my Doctoral degree.

Ich möchte mich auch herzlich bei Frau Frindt bedanken. Vielen Dank dafür, dass Sie an mich geglaubt haben und mir geholfen haben, meine Träume zu erfüllen. "Wo ein Wille ist, ist auch ein Weg". También quisiera agradecer a mi familia, por su amor incondicional. A mis padres Luis y Erika, por darme la vida y estar siempre a mi lado para apoyarme. A mi amado hermano Sebastián, por escucharme, entenderme y siempre recargarme de energía. A mi querida tía Taquitos, por todo su cariño apoyo en cada paso que he dado. A Margarita y abuelitos por su inagotable afecto. Pragnę również podziękować Bogusi i Andrzejowi za przyjęcie mnie w ich domu i za to, że zawsze czuję się jak rodzina. Especialmente quisiera agradecer a Lukas, por todo su amor y apoyo incondicional. Gracias por estar a mi lado, aconsejarme, motivarme y ayudarme a ser la mejor versión de mí misma. Gracias por potenciar cada una de mis habilidades y alentarme a superar mis debilidades. Gracias por ayudarme a ser mejor cada día. 


\section{Table of Contents}

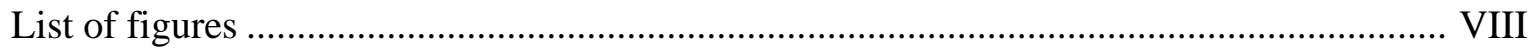

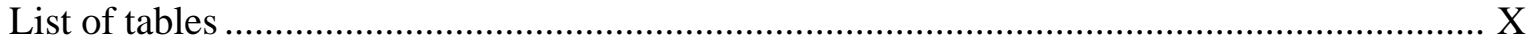

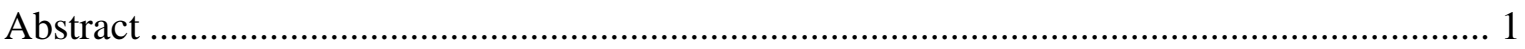

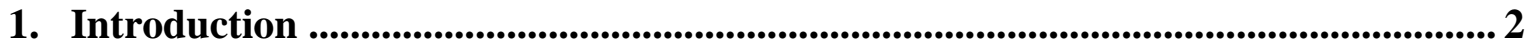

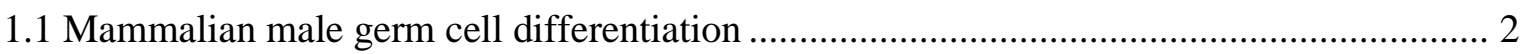

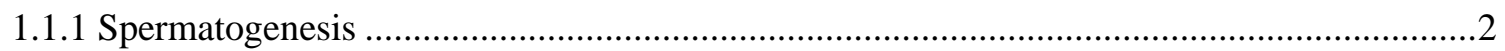

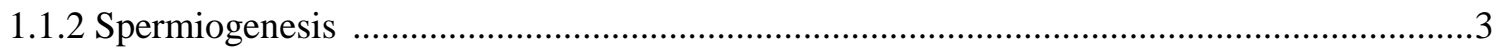

1.2 The head-tail coupling apparatus, a sophisticated structure that anchors the sperm head to tail 6

1.3 Outer dense fiber protein 1 "ODF1" and its role in the formation of the HTCA …......................

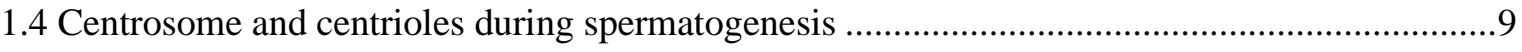

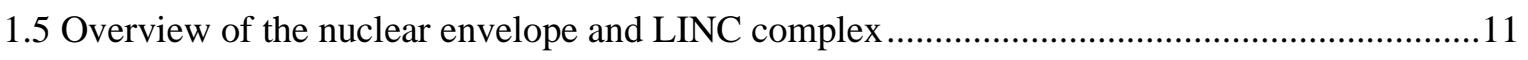

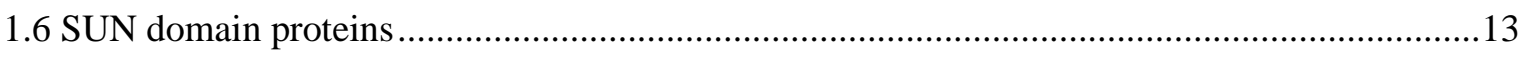

1.7 Male infertility due to acephalic spermatozoa syndrome and its genetic etiology.....................16

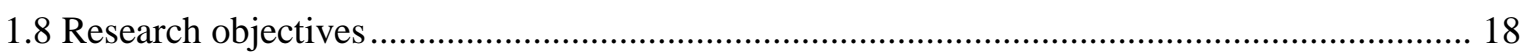

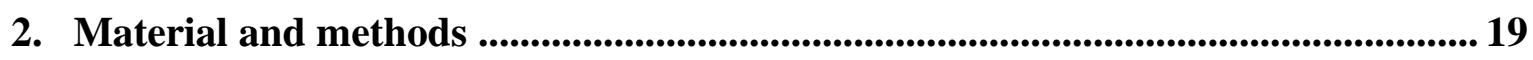

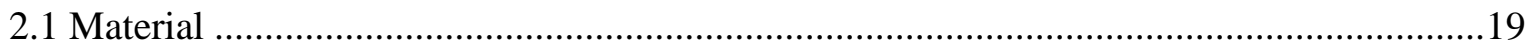

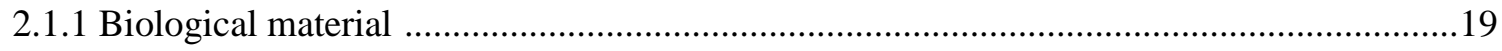

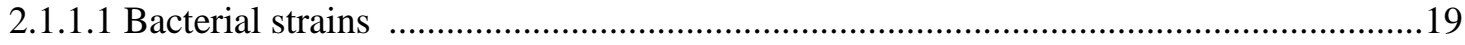

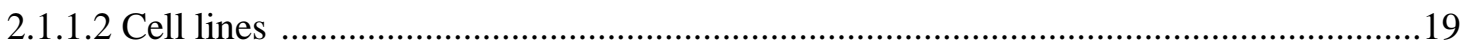

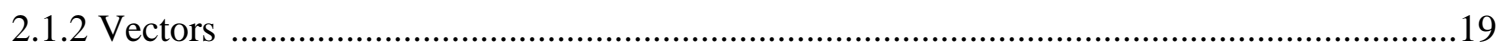

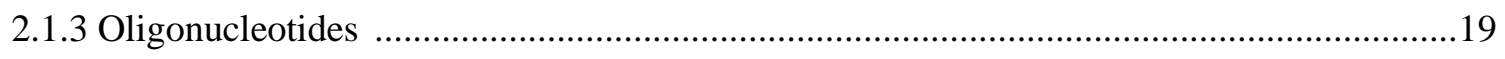

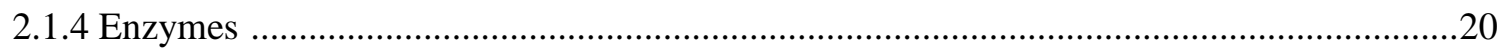

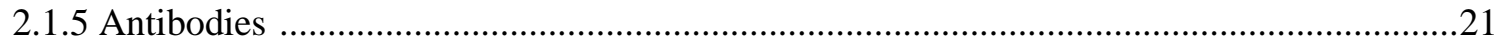

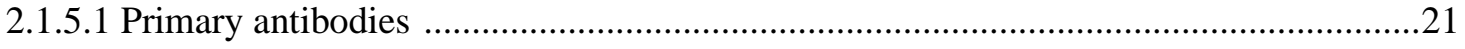

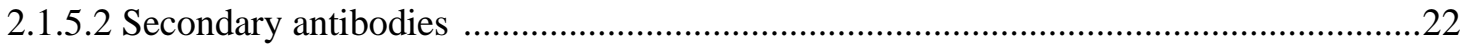

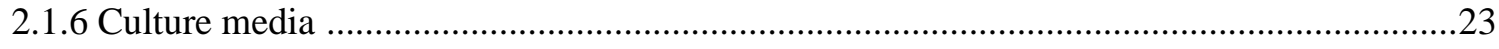

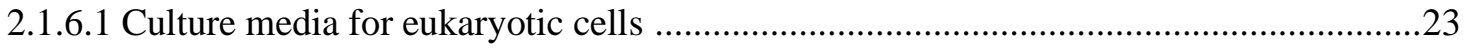

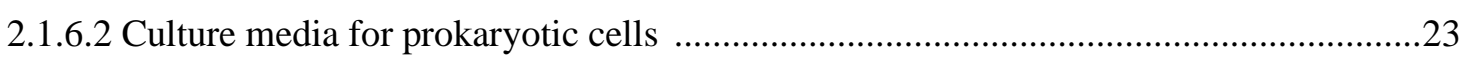

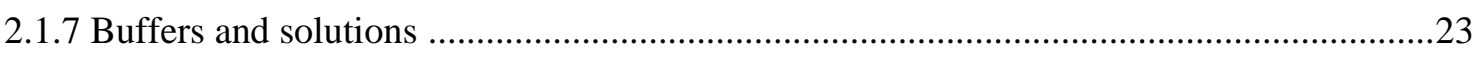

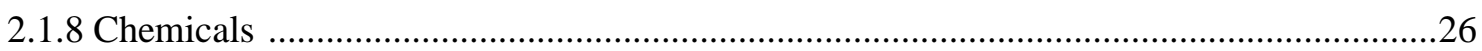




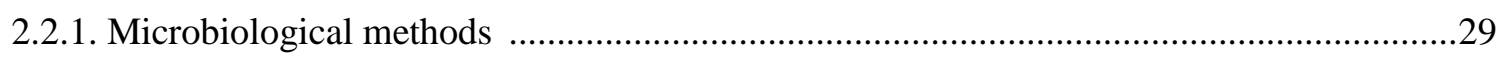

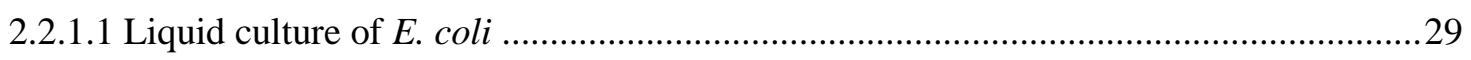

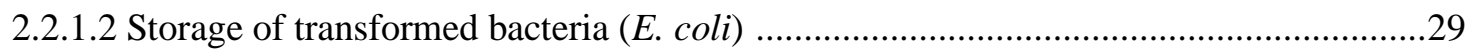

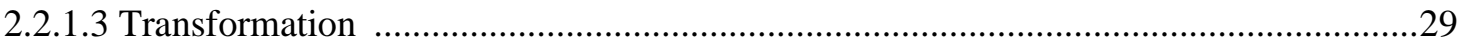

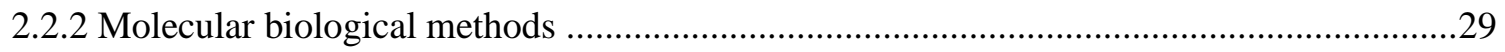

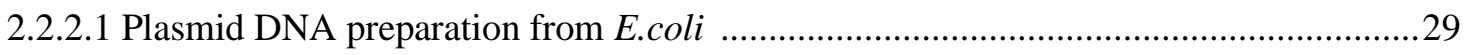

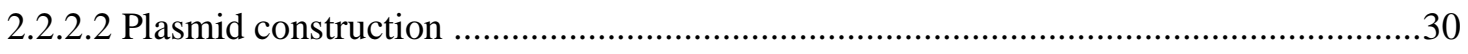

2.2.2.2.1 Subcloning of gene to validate the amber suppression method ...............................30

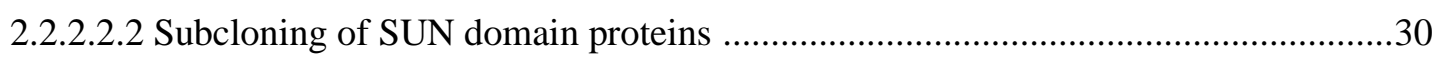

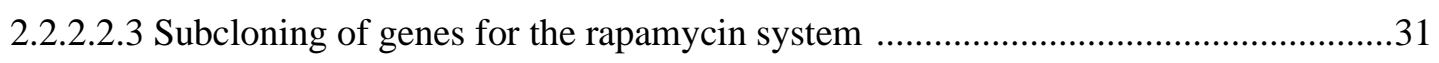

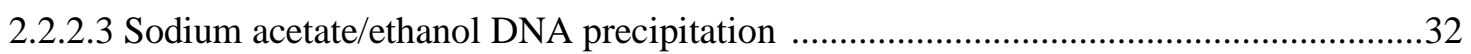

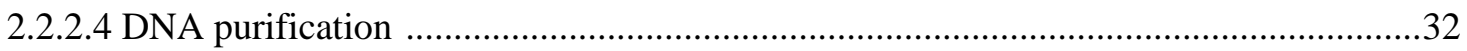

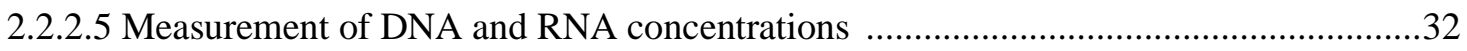

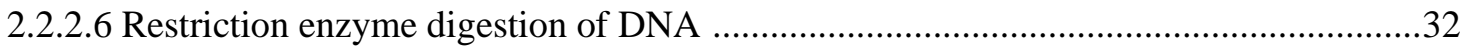

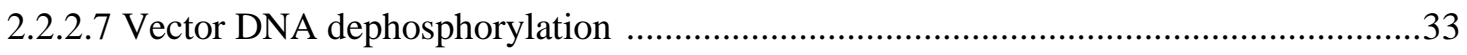

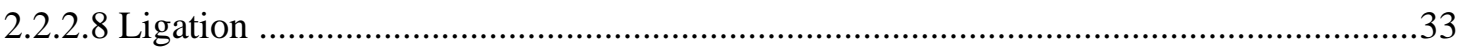

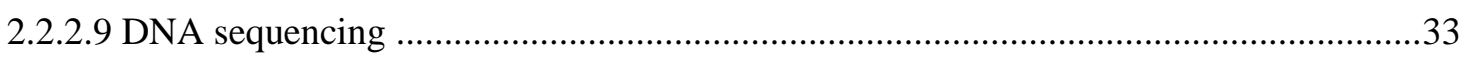

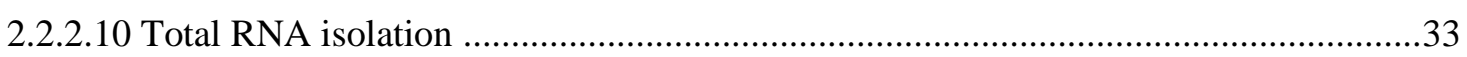

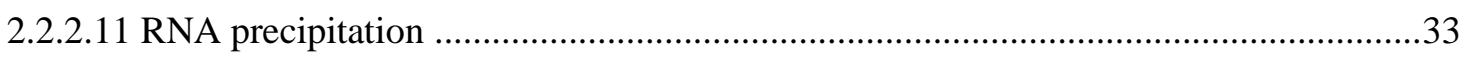

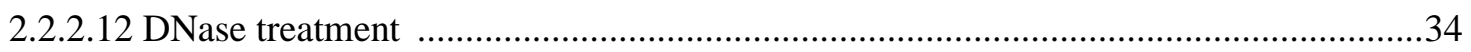

2.2.2.13 Phenol: Chloroform DNA/RNA precipitation .............................................................

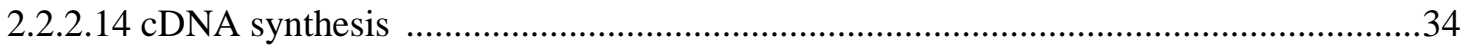

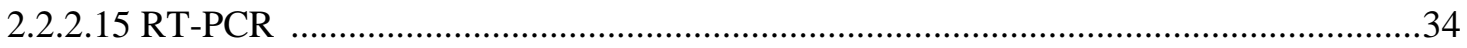

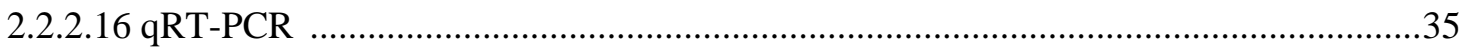

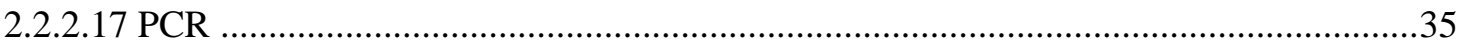

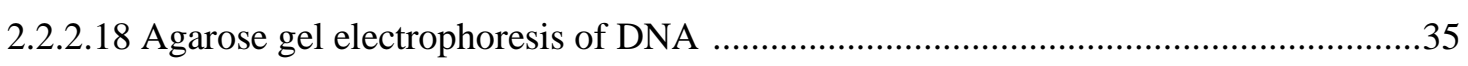

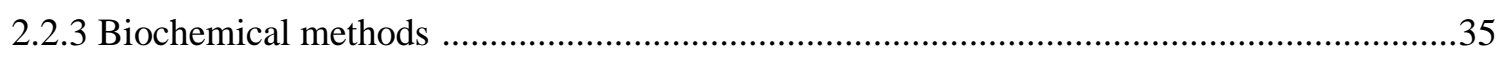

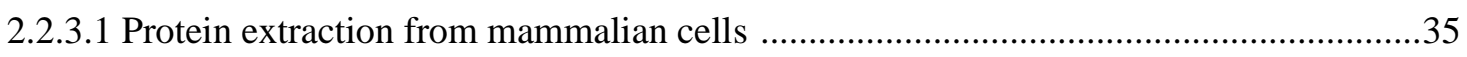

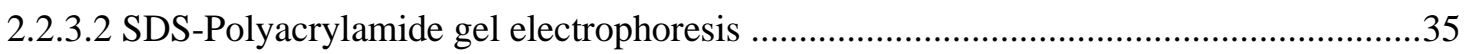

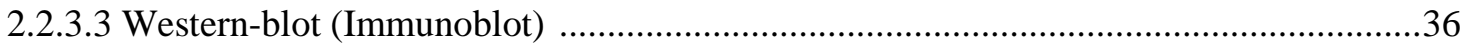

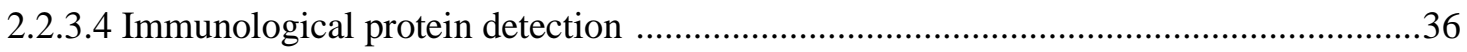

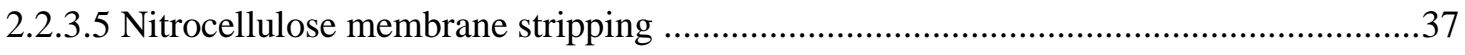

2.2.3.6 Co-IP (Co-immunoprecipitation) …….................................................................... 
2.2.3.7 Crosslinking reaction and harvesting adherent cells

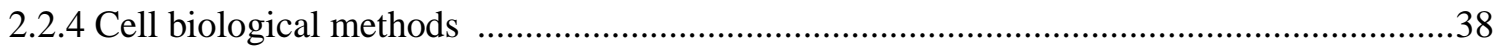

2.2.4.1 Propagation and subculture of eukaryotic cells ............................................................

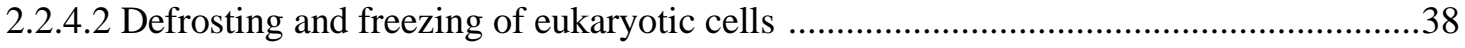

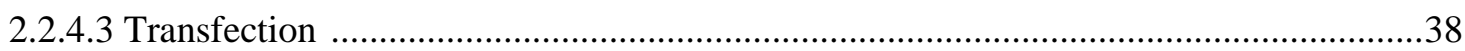

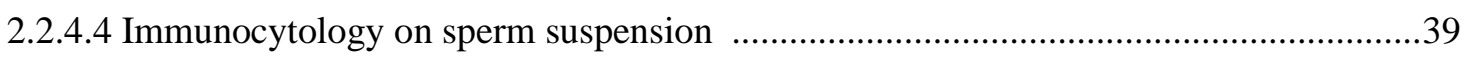

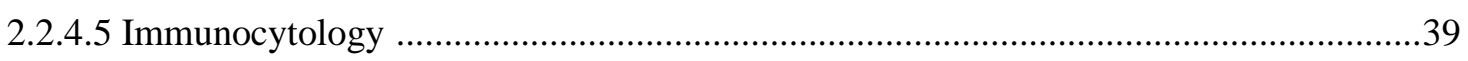

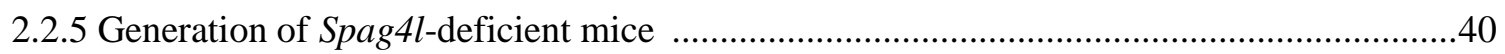

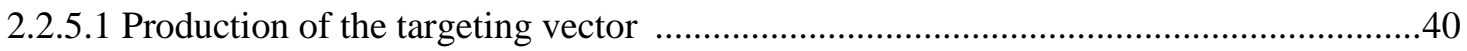

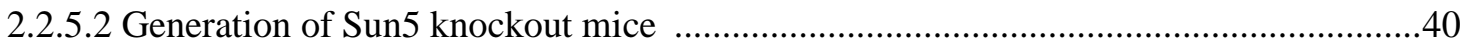

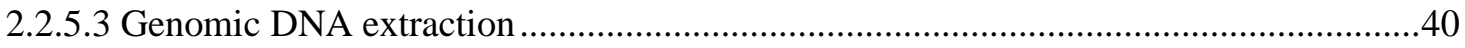

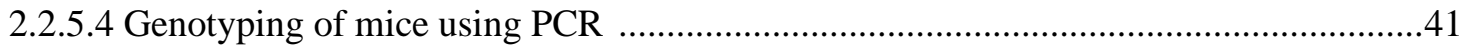

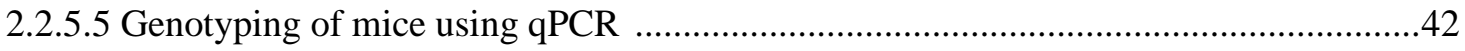

3. Results

3.1 Mutation screening in patients with acephalic spermatozoon phenotype

3.1.1 Patients with decapitated spermatozoa carried normal sequence variations of the gene OdfI compared to the reference wild-type sequence......

3.1.2 Patients with decapitated spermatozoa carried normal sequences of exons 10 and 11 of the gene Hookl compared to the reference wild-type sequence

3.1.3 Patients with decapitated spermatozoa carried normal sequences of exons 6 and 8 of the gene Spag4l compared to the reference wild-type sequence 49

3.2 Characterization of the SUN-domain proteins SPAG4 and SPAG4L . .52

3.2.1 Localization of the SUN domain proteins SPAG4L and SPAG4L2 at the nuclear membrane . .52

3.2.2 Impact of Spag4- deficiency on gene expression during mouse spermatogenesis .57

3.2.3 The amber suppression method and its suitability for the identification of SPAG4 binding proteins

3.2.4 Reevaluation of the topology of SPAG4 and SPAG4L across the nuclear envelope by druginduced protein recruitment . .65

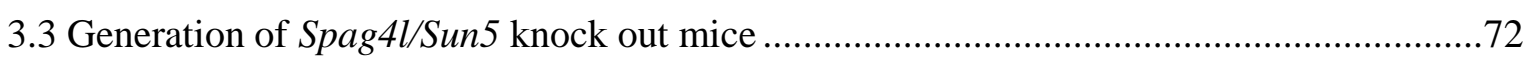

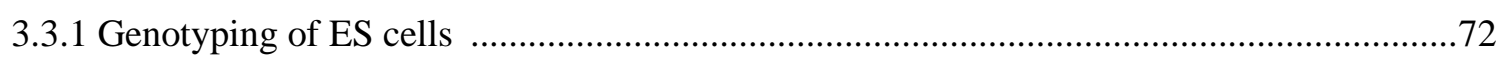

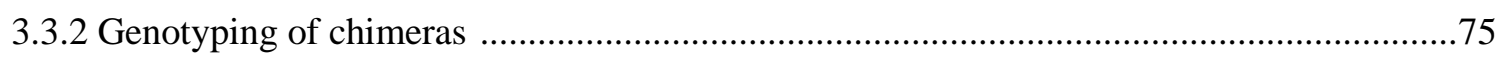

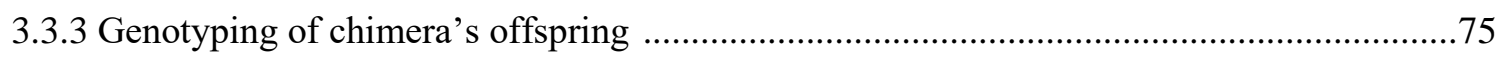

3.4 Identification of proteins that collaborate in the formation of the HTCA ..........................80 
3.4.1 CCDC42 localizes to manchette, HTCA and tail and interacts with ODF1 and ODF2 in the formation of the male germ cell cytoskeleton

3.4.2 The WD40-protein CFAP52/WDR16 is a centrosome/basal body protein and localizes to the manchette and the flagellum in male germ cells . .98

4. Discussion

4.1 Relevance and impact of mutations of candidate genes involved in the linkage of sperm tail to its head.

4.2 Topology and localization of SPAG4 and SPAG4L2 at the nuclear membrane might mediate the connection of the sperm nucleus to its tail

5. References

6. Appendix

6.1 List of Oligonucleotides 144

6.2 List of abbreviations 


\section{List of figures}

Figure 1.1. Scheme of spermatogenesis and spermiogenesis in the mouse

Figure 1.2. Morphogenesis of the spermatozoa during spermiogenesis

Figure 1.3. Scheme of the neck region and head to tail coupling apparatus (HTCA) ...............7

Figure.1.4 Differences of centriolar degeneration in mouse and human ................................11

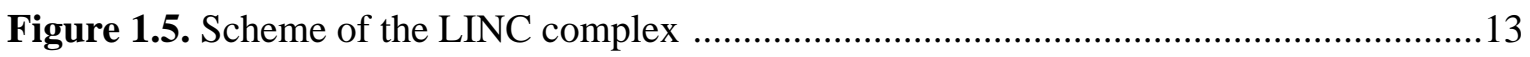

Figure. 1.6. Schematic localization of human SUN5 mutations …........................................15

Figure 3.1. Amino acid sequence alignment of ODF1-exon 1 from patient $n^{\circ} 17$....................45

Figure 3.2. Amino acid sequence alignment of ODF1-exon 1 from patient $n^{\circ} 19$.....................45

Figure 3.3. Amino acid sequence alignment of ODF1-exon 2 from patient $n^{\circ} 17$....................46

Figure 3.4. Amino acid sequence alignment of ODF1-exon 2 from patient $n^{\circ} 19$....................46

Figure 3.5. DNA sequence alignment of Hook 1 -exon 10 from patient $n^{\circ} 17$...........................48

Figure 3.6. DNA sequence alignment of Hook 1 -exon 10 from patient $n^{\circ} 19$...........................48

Figure 3.7. DNA sequence alignment of Hookl-exon 11 from patient $\mathrm{n}^{\circ} 17$ and 19 ...............49

Figure 3.8. Mutations in TM and coiled-coil domain of the human Spag4l ..........................51

Figure 3.9. DNA amplification of exons $6,8,11$ and 13 of Sun5 ......................................51

Figure 3.10. DNA sequence alignment of Sun5-exon6 from patient $\mathrm{n}^{\circ} 17$.............................52

Figure 3.11. DNA sequence alignment of Sun5-exon6 from patient $n^{\circ} 19 \ldots \ldots \ldots \ldots \ldots \ldots \ldots \ldots . . . . .52$

Figure 3.12. Schematic representation of Spag4l2 expression plasmids ................................54

Figure 3.13. The protein domain organization of SPAG4L2 and their individual cellular distribution.

Figure 3.14. Schematic representation of the expression of gene markers during spermatogenesis. 59

Figure 3.15. Gene expression profiling of spermatogenesis marker genes in Spag4-deficient mice

Figure 3.16. Incorporation of the unnatural amino acid pBpa into LMNC sequences ............63

Figure 3.17. Evaluation of the amber suppression method by immunoblotting ......................65

Figure 3.18. Schematic representation of the rapamycin system

Figure 3.19. Rapamycin-induced recruitment of Luciferase-mRFP-FKBP to the nuclear envelope via FRB-ECFP-SUN4 
Figure 3.20. Rapamycin-induced recruitment of Luciferase-mRFP-FKBP to the nuclear

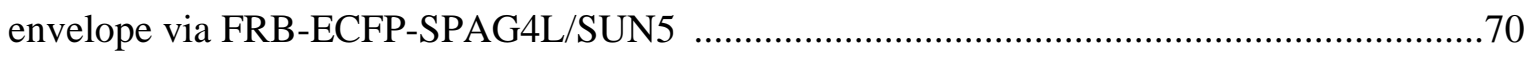

Figure 3.21. Controls for the rapamycin system............................................................ 71

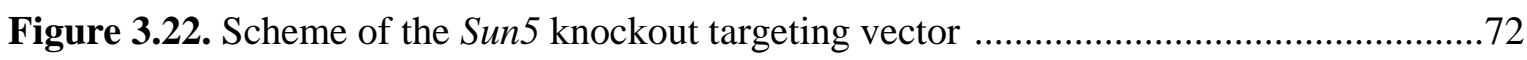

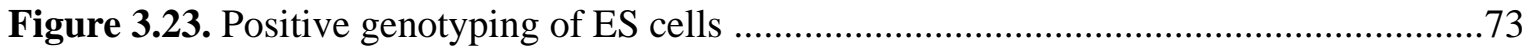

Figure 3.24 Recombinant Sun5 embryonic stem cells are heterozygous for the wild-type allele . .74

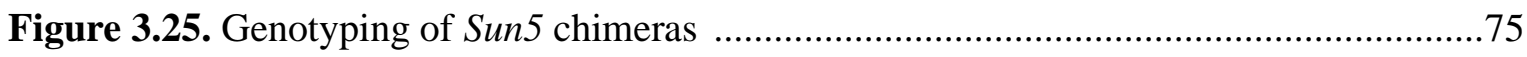

Figure 3.26. Genotyping of the first generation of Spag4l-deficient mice by nested PCR ......76

Figure 3.27. Genotyping of the second generation Spag4l-deficient mice...............................77

Figure 3.28. Breeding scheme to generate homozygous-deficient $\mathrm{Spag} 4 l^{-{ }_{-}}$mice ..................77

Figure 3.29. Relative quantity of exon 7 and 9 of the offspring from heterozygous mice.......78

Figure 3.30. Genotyping example of the fourth generation ................................................

Figure 4.1. Mapping of HTCA protein interaction network ...............................................124

Figure 4.2 Representative scheme of proteins associated with the HTCA in elongating spermatids 125 


\section{List of tables}

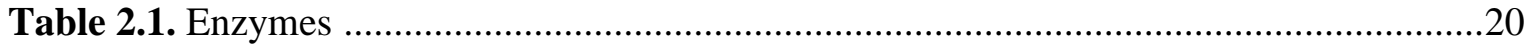

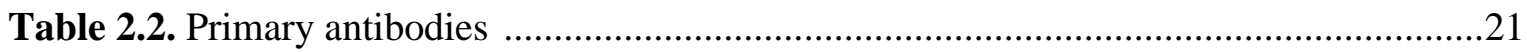

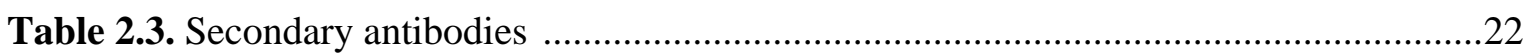

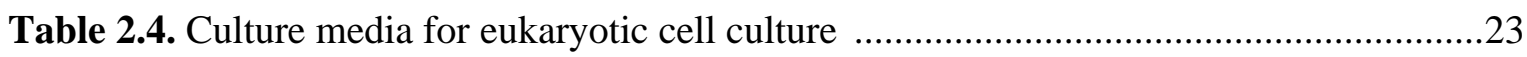

Table 2.5. Culture media for prokaryotic cell culture .......................................................2

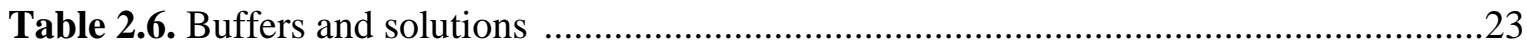

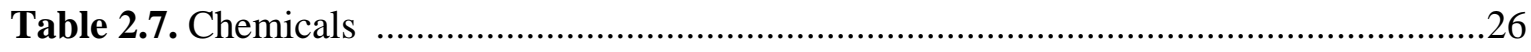

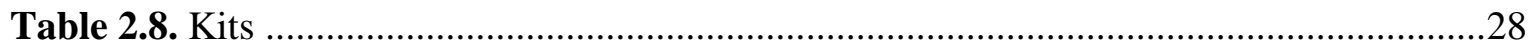

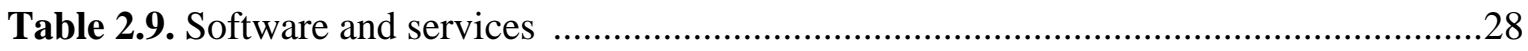

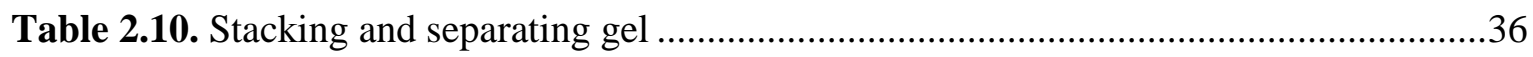

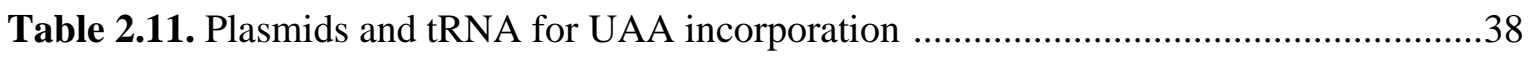

Table 2.12. PCR conditions used for genotyping ............................................................ 41

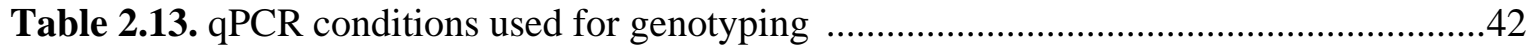

Table 6.1.1 Oligonucleotides for mutation screening in patients with acephalic spermatozoon

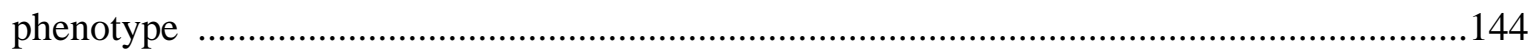

Table 6.1.2 Oligonucleotides for RT-PCR in Spag4-deficient mouse ..................................145

Table 6.1.3 Oligonucleotides for qRT-PCR in Spag4-deficient mouse ................................146

Table 6.1.4 Oligonucleotides for amber suppression method ............................................146

Table 6.1.5 Oligonucleotides for rapamycin system method ............................................147

Table 6.1.6 Oligonucleotides for Spag4l2 investigation .....................................................147

Table 6.1.7 Oligonucleotides for Spag412-deficient mice genotyping .................................148

Table 6.1.8 Oligonucleotides for qPCR for Spag4l2-deficient mice and ES cells ................148 


\begin{abstract}
The head-tail coupling apparatus (HTCA), also known as connecting piece, provides the physical linkage between the sperm head and its tail. The HTCA morphogenesis initiates during spermiogenesis and is assembled at the posterior area of the spermatid nucleus. The HTCA is built around the proximal and the distal centriole, and consists of the accessory structures, basal plate, capitulum, and segmented columns. These structures are responsible for the tight attachment of the sperm head to the tail, and their disruption leads to acephalic spermatozoa syndrome and male infertility. Thus, the stable connection is essential for moving the sperm nucleus towards the oocyte and successful fertilization. However, its molecular composition is scarcely known.
\end{abstract}

The outer dense fiber protein 1 (ODF1) is a component of the HTCA and involved in the tight junction of the sperm head to the tail. Odfl depletion as well as the partial disruption of Hook1 and Spag4l cause sperm decapitation and male infertility in mice. In humans, however, mutations in these genes remain largely unknown. Thus, the DNA from two patients with the acephalic spermatozoa syndrome was screened for mutations. The screen performed in this study reveals a normal Odf1 gene sequence. Besides, exons 10 and 11 of Hookl and exons 6 and 8 of Spag $4 l$ were identical to the wild type variant. Therefore, the analyzed regions of the genes Odf1, Hook1, and Spag4l seem not to be implicated in the acephalic syndrome suffered by the two patients here studied.

Beyond that, the interaction between ODF1 and the nuclear membrane protein SPAG4 has been reported as a novel male germ cell-specific linkage complex between the cytoskeletal connecting piece and the nuclear membrane, although their mechanism of interaction is unclear. Thus, the induction of dimerization by rapamycin was explored as a new tool to analyze SUN4 and SUN5 topology. Besides ODF1 or SPAG4 interacting proteins that collaborate in the formation of the HTCA have not been identified. In this study, the coiled-coil domain containing 42 (CCDC42), another essential HTCA component that causes decapitation when missing, and the cilia and flagella associated protein 52 (CFAP52) have been characterized in detail. CCDC42 has been localized to the manchette, perinuclear ring, and tail in male germ cells. CCDC42 interacts biochemically with the outer dense fiber proteins ODF1 and ODF2. These interactions might contribute to the assembly of proteins required for the formation and the stability of the HTCA. CFAP52 localizes to the manchette and sperm tail, indicating that CFAP52 is another promising candidate for the HTCA interacting network. 


\section{Introduction}

\subsection{Mammalian male germ cell differentiation}

\subsubsection{Spermatogenesis}

Spermatogenesis is a sophisticated process whereby diploid spermatogonia generate mature haploid spermatozoa. This process consists of three successive phases known as the mitotic or proliferative phase, meiotic phase, and cytodifferentiation phase (Fig. 1.1) (Russell, 1990).

Stem cells multiply during the mitotic phase and undergo differentiation to produce spermatogonia type A. This transformation process continues until spermatogonia type B are generated. Intermediate type B spermatogonia undergo mitosis to give rise to preleptotene spermatocytes (Pl) (Oakberg, 1956; De Rooij \& Grootegoed, 1998; Eddy, 1998).

The meiotic phase includes two successive rounds of cell divisions to generate haploid spermatids (Hermo et al., 2010). Spermatocytes undergo two meiotic divisions that include nuclear changes required for chromosome recombination and genetic material exchange. The first meiotic division is characterized by a prolonged prophase which includes leptotene, zygotene, pachytene, diplotene, and diakinesis. Chromatin condensation is initiated during leptotene and is followed by chromosome pairing (synaptonemal complex formation) in zygotene (Clermont, 1972; Russell, 1990). During zygotene-pachytene, the chromosome synapsis is regulated by meiotic proteins of the synaptonemal complex (Meuwissen et al., 1992; Yuan et al., 2000; Costa et al., 2005). After chromosome pairing, homologous chromatids exchange genetic material in pachytene, a process known as crossing-over. Afterward, the synaptonemal complex disintegrates in the diplotene phase. With diakinesis, the first meiotic division is completed, and secondary spermatocytes are generated. Secondary spermatocytes undergo a fast second meiotic division without DNA replication to produce round spermatids (Russell, 1990).

Spermiogenesis, or cytodifferentiation phase, is the final stage of mature spermatozoa production. During this phase, round spermatids experience a dramatic remodeling that includes nuclear shaping and condensation, acrosome and flagellum development, and cytoplasm elimination, which results in a functional spermatozoon (Russell, 1990). 


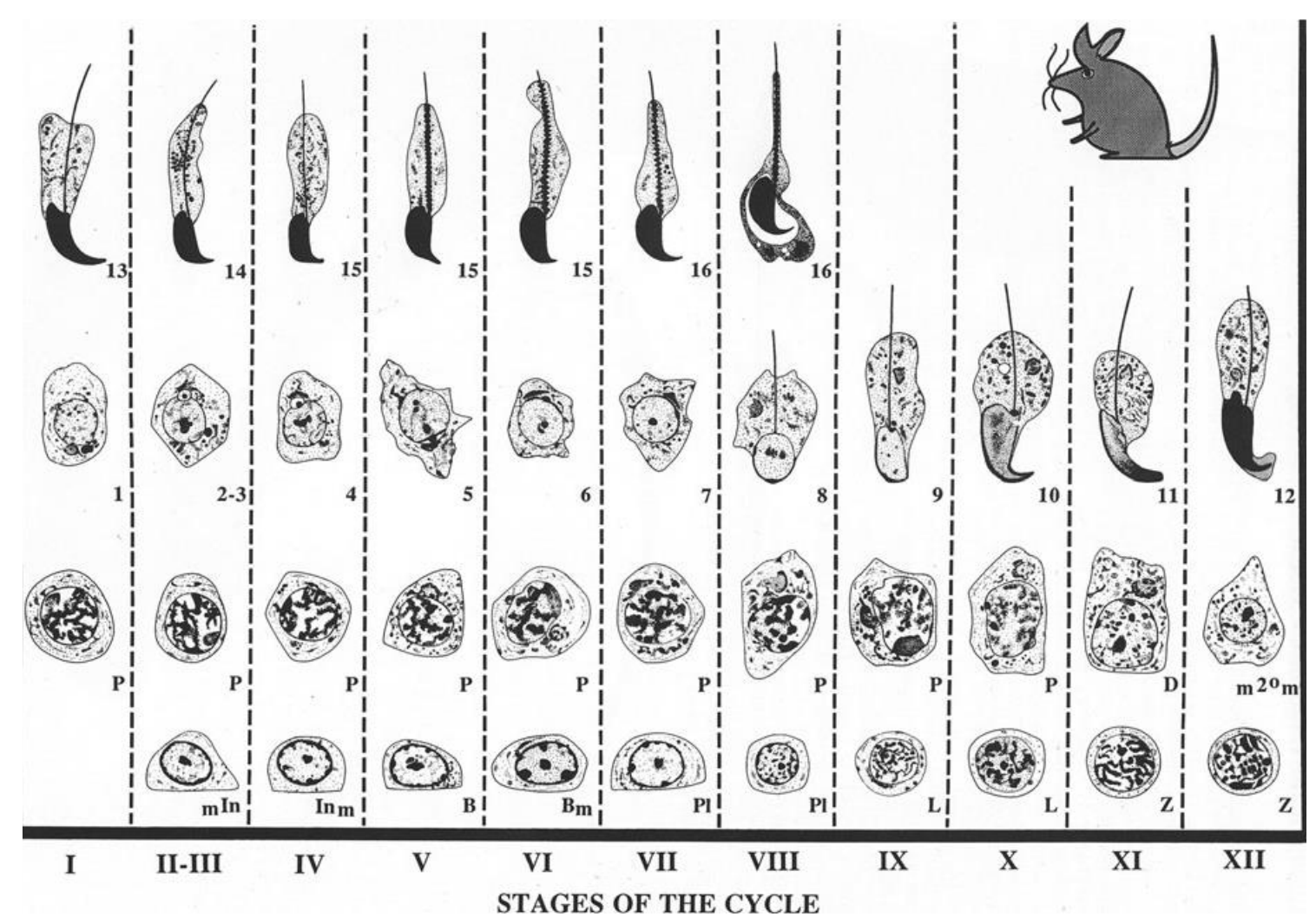

Figure 1.1 Scheme of spermatogenesis and spermiogenesis in the mouse. $\mathrm{m}^{\mathrm{In}}$ (mitosis of intermediate spermatogonia), ${ }^{\mathrm{In}} \mathrm{m}$ (intermediate spermatogonia), B (spermatogonia), Bm (mitosis of spermatogonia), $\mathrm{Pl}$ (preleptotene), L (leptotene), Z (zygotene), ( $\mathrm{P}$ (pachytene), D (diplotene), $\mathrm{m} 2^{\circ} \mathrm{m}$ (second meiotic division), 1-8 (round spermatids), 9-11 (elongating spermatids), 16 (late spermatids) (Russell, 1990).

\subsubsection{Spermiogenesis}

Spermiogenesis is the last phase of spermatogenesis, where round spermatids experience a total transformation to evolve into mature spermatozoa (Clermont, 1972). This process starts with the acrosome development which consists of four stages: (1) Golgi phase, (2) cap phase, (3) acrosome phase, and (4) maturation phase. During the Golgi phase (Fig.1.2-A), the Golgi apparatus produces several proacrosomal vesicles. The fusion of these vesicles forms the acrosomal vesicle that afterward attaches to the nuclear envelope by the acroplaxome plate. Moreover, in the first phase of the acrosome development, centrioles localize close to the Golgi apparatus. However, centrioles migrate to the caudal area of spermatids during the Golgi phase, being opposite to the acrosome. Thus, acrosome and centrioles establish the bipolarity of the nucleus (Hermo et al., 2009b; Chemes, 2012). Further, the proximal centriole attaches to the nucleus, articulating the implantation fossa and forming the connecting piece. On the other hand, the distal centriole gives rise to the basal body and initiates the formation of the axoneme (Fawcett, 1975; Chemes \& Rawe, 2010; Hoyer-Fender, 2012). 
Afterward, during the cap phase (Fig. 1.2-B) the acrosome increases in size and moves anteriorly. The development of the manchette characterizes the acrosomal phase (Fig. 1.2-C). This transient microtubular structure evolves from the perinuclear ring situated in the caudal area of the acrosome-acroplaxome complex (Rattner \& Olson, 1973). The manchette extends its microtubules longitudinally to the elongating spermatid. This transient structure provides a platform for intramanchette transport (IMT), where molecular motors transport essential proteins to develop the flagellum. In the late stages of spermiogenesis, the manchette disassembles (MacKinnon et al., 1973; Kierszenbaum et al., 2003; Kierszenbaum \& Tres, 2004; O'Donnell \& O'Bryan, 2014).

During the maturation phase (Fig. 1.2-D), the manchette disintegrates, and the sperm nucleus condensates by compaction of the chromatin. During condensation, histone and nonhistone proteins are replaced first by transition proteins, which are finally replaced by protamines. Once spermatids have reduced their volume, the excess of cytoplasm (residual body) is eliminated. The residual cytoplasm is phagocytized and digested by the Sertoli cells (Balhorn, 1982; Russell, 1990).

At the end of the maturation phase, spermatozoa are completely formed and are composed of two main structures: the head and the tail. The flagellum is constituted by the axoneme, a central microtubular structure (9+2 arrangement), and is further subdivided into the middle, the principal, and the end piece. The axoneme in the flagellum is encompassed by outer dense fibers (ODFs), which in turn are covered by mitochondria in the midpiece. The principal piece is composed of ODFs, which are surrounded by a fibrous sheath (Russell, 1990; O'Donnell \& O'Bryan, 2014). ODFs are responsible for protecting the sperm tail from tensile forces during epididymal passage and ejaculation (Baltz et al., 1990a; Lindemann, 1996). 

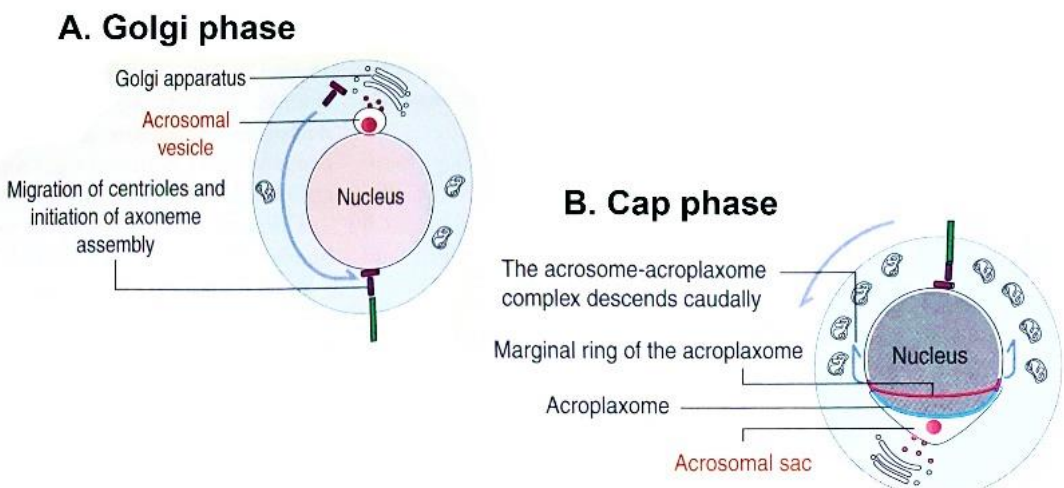

\section{Acrosomal phase}

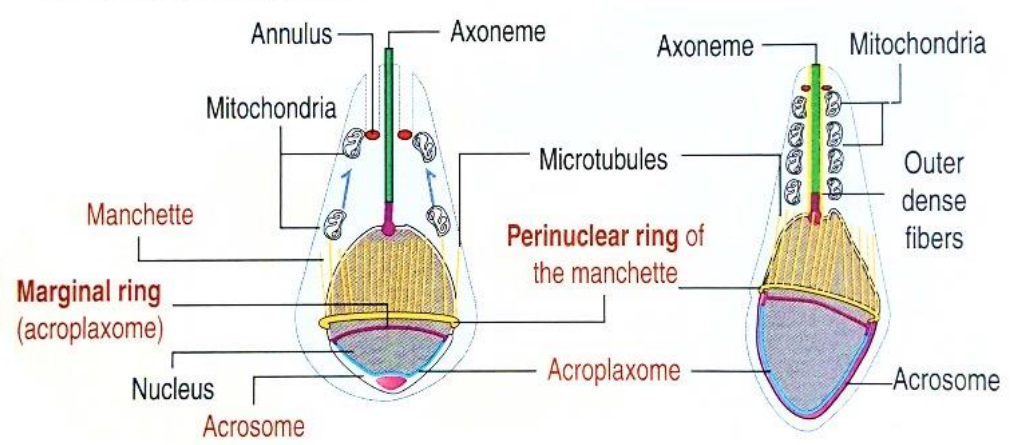

\section{Maturation phase}

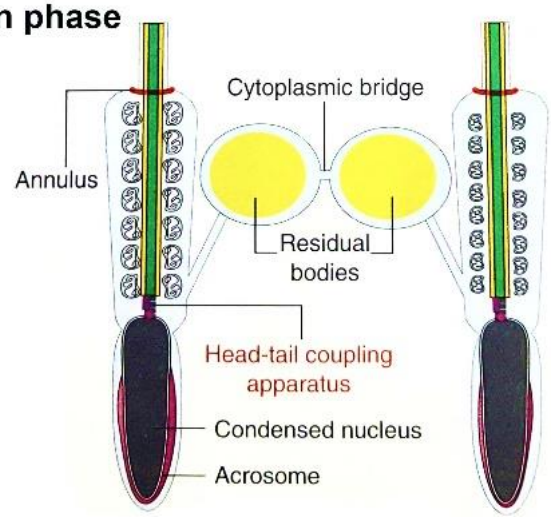

Figure 1.2. Morphogenesis of the spermatozoa during spermiogenesis. (A) Golgi phase: Golgi apparatus gives rise to proacrosomal granules, which are transported to the acrosomal vesicle. Centrioles migrate to the caudal area of the nucleus and initiate the axoneme assembly. (B) Cap phase: the acrosome starts to descend to the caudal area and increases its size. (C) Acrosomal phase: the acrosome attaches to the acroplaxome, and the manchette extends its microtubules. Outer dense fibers and mitochondria are aligned around the axoneme. (D) Maturation phase: chromatin is condensed by replacement of transition proteins, manchette is disintegrated, and residual bodies are eliminated (adapted from Histology and Cell Biology: An Introduction to Pathology - 4th Edition, (92016.) 


\subsection{The head-tail coupling apparatus, a sophisticated structure that anchors the sperm head to tail.}

The head-tail coupling apparatus (HTCA), also known as the connecting piece or neckpiece, is a sophisticated structure that anchors the head to the tail in sperm. The HTCA consists of sperm centrioles and several proteins that contribute to building a tight connection between the head and flagellum (Chemes \& Rawe, 2010). The connecting piece, a complex dense structure is placed near to centrioles. It starts to organize in striated longitudinal columns and assemble the capitulum (Fawcett \& Phillips, 1969). The capitulum is a curved shape structure that conforms to the concavity of the basal plate. Although a narrow space separates both structures, they are bridged by fine filaments. The basal plate further establishes a shallow concave area in the posterior pole of the nucleus, denominated implantation fossa (Fawcett, 1975). In this place, the implantation fossa is in contact with the capitulum, segmented columns of the neckpiece, and the proximal centriole. Thus, these structures assemble the attachment of the sperm head to the tail (Fig. 1.3) (Chemes et al., 1999).

The proper assembly of the HTCA in sperm is required to fertilize oocytes successfully. Therefore, abnormalities in the HTCA lead to acephalic spermatozoa syndrome (Perotti et al., 1981; Chemes et al., 1987). Studies in patients with headless spermatozoa evidence different abnormalities in the head-tail junction, inter alia, loss of the implantation fossa, basal plate, and the presence of nuclear envelope-derived vesicle in the caudal pole. Hence, alterations in these structures lead to either fragility or the total disruption of the tight head-tail conjunction (Baccetti et al., 1989; Chemes et al., 1999; Toyama et al., 2000). Nonetheless, a deeper characterization and comprehension of the morphological and molecular components of the HTCA remains to be elucidated. 

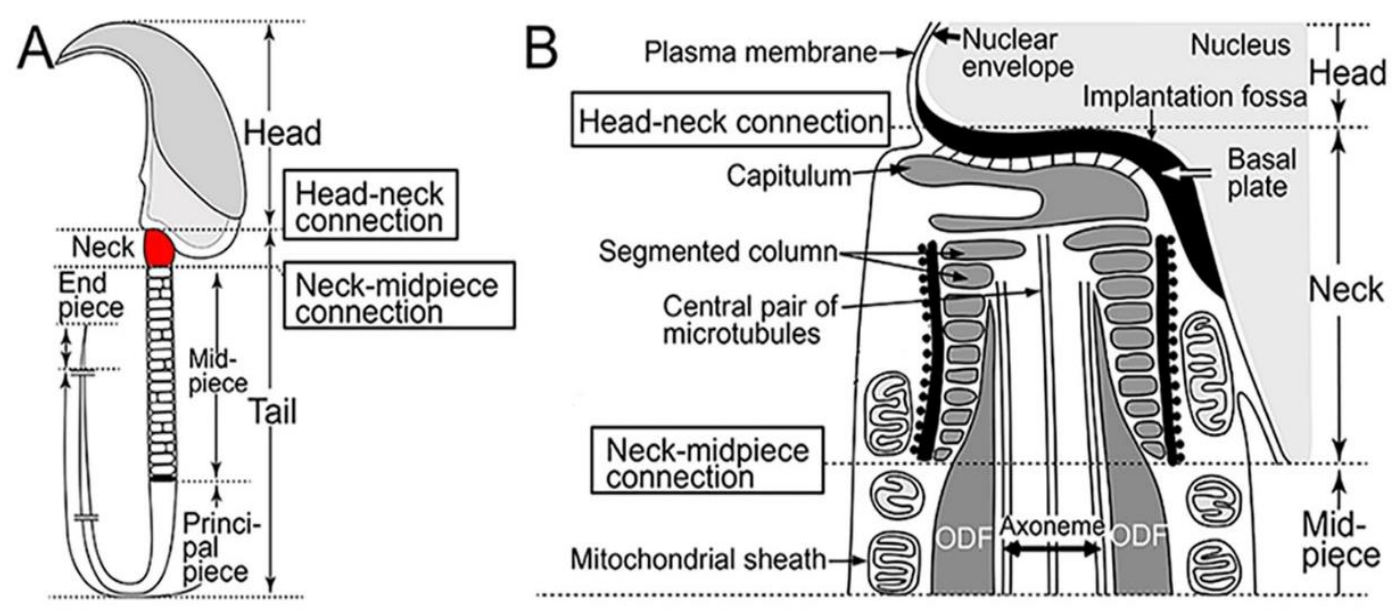

Figure 1.3. Scheme of the neck region and head to tail coupling apparatus (HTCA). (A) Neck localization in a mature spermatozoon. (B) A detailed description of the neck components and HTCA. The implantation fossa is a shallow concave area formed by the basal plate in the caudal area of the nucleus. The HTCA structure includes the basal plate that is attached to the capitulum, centrosome and segmented columns connected to the outer dense fibers (ODFs). ODFs are surrounded by the mitochondrial sheath (adapted from Ito et al., 2019).

\subsection{Outer dense fiber protein 1 "ODF1" and its role in the formation of the HTCA}

The sperm flagellum arises from the distal centriole at the posterior region of the nucleus. The centriole gives rise to the axoneme, which consists of two central microtubules surrounded by nine doublet microtubules. In the mid-piece, the axoneme is surrounded by nine outer dense fibers (ODFs). Additionally, the connecting piece and segmented columns are continuous to the capitulum, whereas their posterior area overlaps with the nine ODFs. ODFs in the mature flagellum extend through almost all the length of the principal piece (Fawcett, 1975). Outer dense fibers are indispensable for flagellar movements because they provide elastic recoil and further protection against shearing forces during epididymal transport and ejaculation (Baltz et al., 1990). Moreover, they provide force transmission to the flagellar base during the axonemal beat (Lindemann, 1996).

ODFs are composed of around 14 polypeptides; however, few of them have been described (Vera et al., 1984; Oko, 1988; Petersen, 1999). ODF1 is a spermatid-specific protein of 27 $\mathrm{kDa}$. It consists of an $\mathrm{NH}_{2}$-terminal, which enables self-interaction, and a highly conserved $\mathrm{COOH}$-terminal. The $\mathrm{N}$-terminal domain contains a putative leucine zipper dimerization motif, whereas the $\mathrm{C}$-terminal comprises a high content of cysteine and proline as a repetitive tripeptide 
motif CXP (van der Hoorn et al., 1990; Burfeind \& Hoyer-Fender, 1991; Morales et al., 1994; Shao \& van der Hoorn, 1996). However, the CXP motif variability is not responsible for male infertility in humans (Hofferbert et al., 1993).

ODF1 shares structural features with small heat shock proteins (sHSPs), particularly with the conserved $\alpha$-crystalline domain being named HSPB10 for this reason (Fontaine et al., 2003). ODF1/HSPB10 is restricted to the testis and its expression is first detectable in the round spermatid stage (Burmester \& Hoyer-Fender, 1996). Given that ODF1 localizes in the sperm connecting piece, this protein acquires relevance regarding its role in the HTCA stability, the formation of the sperm tail, and male fertility. The depletion of ODF1 in mice results in male infertility because of several abnormalities of the HTCA. These disorders include the abnormal structural organization of mitochondrial sheath and ODFs, and destabilization of the connection between the nuclear membrane and capitulum by incrementing their distance, resulting in sperm decapitation. Thus, ODF1 ablation severely affects the stability of the connecting piece and may also cause human male infertility (Yang et al., 2012; 2014).

Although ODF1 is an essential component of the connecting piece, few ODF1 interacting proteins have been identified. ODF1 can self-associate due to its leucine zipper motif in the Nterminal region, albeit this interaction is fragile (Shao \& van der Hoorn, 1996). Beyond that, ODF1 leucine zipper enables its interaction with a major ODF protein of the sperm tail accessory fibers, the outer dense fiber protein 2 (ODF2) (Shao et al., 1997). ODF1 and ODF2 reside in the head-connecting piece complex. Therefore, the presence of these two proteins in the connecting piece might be involved in the stable junction of the head to tail in sperm (Schalles et al., 1998).

The stable linkage between the nucleus to the sperm tail is mediated by the HTCA, which is supported by several molecular components that are still unknown. This linkage seems to be assisted by the SUN domain proteins, which tether the centrosome to the nuclear membrane (Malone et al., 2003). The SUN domain protein SPAG4 localizes in the posterior pole of the sperm head in round and elongating spermatids and seems to interact with ODF1 (Shao et al., 1999; Kierszenbaum \& Tres, 2002; Pasch et al., 2015). Nevertheless, the disruption of ODF1 does not affect the localization of SPAG4. Although SPAG4 is an essential component for the proper sperm head formation, manchette assembly, and male fertility, its disruption does not prevent the HTCA formation in mice. However, SPAG4 is required for the attachment of the connecting piece to the nucleus. The interaction of ODF1 and SPAG4 may mediate the headtail connection. In this scenario, the nuclear membrane protein SPAG4, which resides at the posterior pole of spermatids, interacts with ODF1 that localizes in the basal plate and capitulum. 
Thus, SPAG4 might recruit ODF1 to the nuclear membrane and arrange the tight linkage of the sperm head to the tail (Calvi et al., 2015; Pasch et al., 2015; Yang et al., 2018). Likewise, the SUN domain protein SPAG4L is expressed in elongating spermatids and strongly associated with the ODF1 leucine zipper (Shao et al., 2001; Fitzgerald et al., 2006). However, the interaction of ODF1 with the SUN domain proteins remains to be investigated.

Furthermore, additional ODF1 interacting proteins might participate in the linkage of the sperm head and tail, inter alia, KLC3 (kinesin light chain 3), which resides in the midpiece of the sperm tail, and OIPI, a member of the RING finger family (Bhullar et al., 2003; Zarsky et al., 2003). Besides that, the coiled-coil domain containing 42 (CCDC42) has a reported function in the HTCA and sperm flagellum development. Mice lacking $C c d c 42$ display an abnormal HTCA conformation, prevent sperm flagella formation, and suffer from male infertility (Pasek et al., 2016). Therefore, CCDC42 is an ideal candidate to evaluate its participation in the physical linkage of the sperm head to the tail.

\subsection{Centrosome and centrioles during spermatogenesis}

The centrosome is the main microtubule-organizing center (MTOC) and is responsible for generating the mitotic spindle during cell division. It consists of two small organelles, known as centrioles, embedded in a dense pericentriolar matrix (PCM). The PCM is composed of several proteins, although the most abundant components are $\gamma$-tubulin and pericentrin (Kellogg et al., 1994; Bornens, 2002; Łuksza et al., 2013).

The centrosome is an example of semi-conservative segregation (Kochanski \& Borisy, 1990). Duplication starts in the transition phase G1, where each cell contains a centrosome consisting of two centrioles. This process finishes with the separation of the two centrosomes and their associated PCM. Each centrosome contains a pair of centrioles that consist of a mature or mother centriole and a young or daughter centriole (Urbani \& Stearns, 1999; Zimmerman et al., 1999).

Centrioles are short cylindrical structures with $\sim 0.5 \mu \mathrm{m}$ in length and $0.2 \mu \mathrm{m}$ in diameter perpendicularly oriented to each other. They are mostly built from $\alpha$ - and $\beta$-tubulin and exhibit an organized structure of nine microtubule triplets with a proximal, middle, and distal tubule (Vorobjev \& Chentsov, 1980; Urbani \& Stearns, 1999).

The mature or mother centriole consists of distal and subdistal appendages (Vorobjev \& Chentsov, 1982; Alvey, 1985; Hoyer-Fender, 2010). The mature centriole in eukaryotic cells 
becomes the basal body, which localizes at the base of cilia and flagella (Kellogg et al., 1994; Urbani \& Stearns, 1999). In male germinal cells, the centriolar pair migrate from the Golgi apparatus region to the distal pole of the nucleus. There, the proximal centriole participates in the formation of the connecting piece and the anchorage of the sperm nucleus and its tail, whereas the distal centriole acts as a basal body and gives rise to the axoneme (Fawcett \& Phillips, 1969; Chemes \& Rawe, 2010).

During spermiogenesis, the pericentriolar material (PCM) is remodeled and replaced by striated columns and the capitulum, which connect the sperm head to the tail. The proximal and distal centrioles attach to the nucleus via the HTCA. The proximal centriole is tightly connected with the capitulum, whereas the distal centriole initiates the axoneme formation. In turn, the striated columns connect with the nine outer dense fibers at the lower portion of the neck region (Fawcett \& Phillips, 1969; Iwashita \& Oura, 1980). During this process the distal centriole that gives rise to the axoneme disintegrates, whereas the proximal centriole goes through a reduction process. The reduction process varies between species. In mice both centrioles are disintegrated, whereas in humans and rhesus monkeys centrosome degeneration is partial (Fig. 1.4) (Schatten et al., 1986; Manandhar \& Schatten, 2000).

During the proximal centriole reduction some centriolar proteins are dismissed (Schatten et al., 1986; Manandhar et al., 1998). However, further proteins have been localized in the connecting piece, inter alia, Centrobin, SPATA6, and SPAG4. Beyond that, the depletion of these proteins severely affects the HTCA, and hence they are directly related to the acephalic spermatozoa syndrome (Liška et al., 2009; Yassine et al., 2015; Yuan et al., 2015; Shang et al., 2017; Yang et al., 2018). Therefore, these proteins play an essential role in the development and stabilization of the HTCA. Furthermore, disturbances in the formation of the HTCA are observed, derived from the misalignment or unsuccessful attachment of centrioles to the spermatid nucleus. Besides that, centrosomal defects might interfere with the success of assisted reproduction. In intracytoplasmic sperm injection (ICSI), the sperm is microinjected into the oocyte. However, the microinjection of a sperm with a faulty centriole affects early embryonic development and implantation.

Moreover, centriolar defects may have a genetic cause inherited to the progeny causing male infertility later on. Thus, centriolar impairment seriously affects HTCA stability and weakens the linkage of the sperm head to tail, causing detachment. The sperm head-to-tail linkage comprises centrioles and connecting piece. In some cases of male infertility, decapitated spermatozoa are produced by abnormalities in sperm structure. These include malformation of the midpiece, absence of the implantation fossa or basal plate (Kamal et al., 1999). The 
remodeling process of male germ cells during spermiogenesis includes centrosome reduction and protein redistribution. The pericentriolar material surrounding centrioles is transformed into the capitulum and striated columns (Avidor-Reiss et al., 2019). These structures contain specific proteins such as SPATA6, essential for the head-to-tail junction (Yuan et al., 2015). However, the set of proteins involved in the HTCA formation remain to be identified. Abnormalities of the mid-piece in sperm could affect fertilization potential and impair the embryo development after intracytoplasmic sperm injection (Kamal et al., 1999). Hence, identifying the molecular components of the HTCA is of great significance for the development of new diagnostic tools and the treatment of patients with acephalic spermatozoa (Simerly et al., 1995; Schatten \& Sun, 2009; Chemes, 2012).

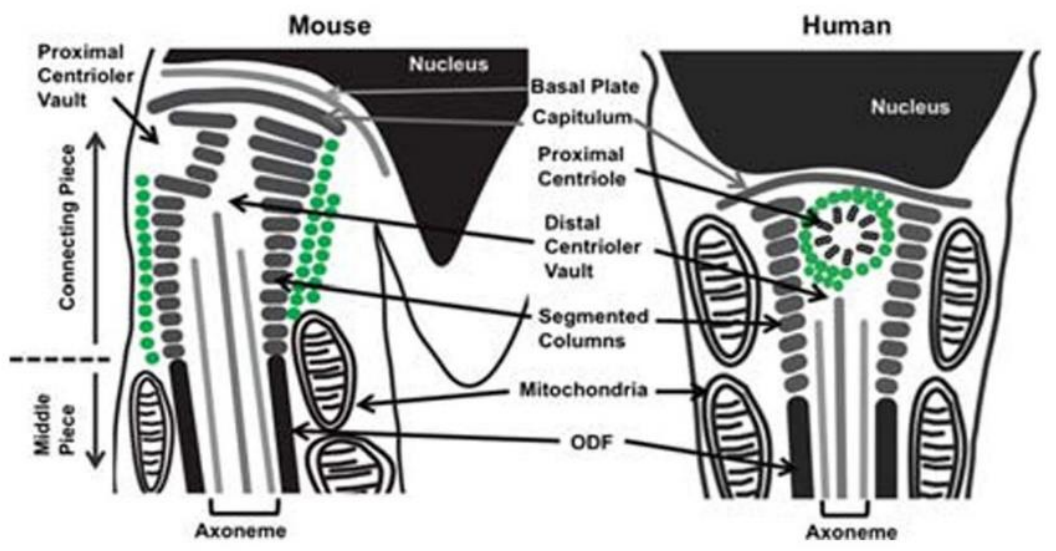

Figure 1.4 Differences of centriolar degeneration in mice and humans. In mice, distal and proximal centrioles degenerate, and this area is occupied by dense material. The centriolar vault is surrounded by segmented columns and in the posterior area of the distal vault, the axoneme emerges, which is surrounded by ODFs. In humans and rhesus monkeys only the distal centriole disassembles. The proximal centriole is in close contact with the capitulum and is associated with striated columns. The distal centriole gives rise to the axoneme, which is surrounded by ODFs (Image from Goto et al., 2010).

\subsection{Overview of the nuclear envelope and the LINC complex}

The nuclear envelope is a selective barrier responsible for separating the nucleus from the cytoplasm. It is constituted by an inner and an outer membrane (INM, ONM), enclosing the perinuclear space (PNS). The INM and ONM are fused at the nuclear pores, which are responsible for mediating the traffic of molecules between the cytoplasm and the nucleus (Kite, 1913; Watson, 1955; Gerace \& Burke, 1988). The INM is predominantly associated with lamins type A and B, and is involved in chromatin organization. The genes Lmna, Lmnb1, and Lmnb2, 
encode lamins. Lmna encodes, by alternative splicing, the principal isoforms A and C. Lmnb1 encodes lamin B1, whereas Lmnb2 gives rise to the isoforms B2 and B3. Lamin A/C is expressed in later somatic cell differentiation stages, whereas lamins B3 and C2 are shorter splicing variants expressed exclusively in the early stages of spermatogenesis. Furthermore, lamin C2 is exclusively expressed in spermatocytes while lamin B3 is spermatid specific (Watson, 1955; Rober et al., 1989; Furukawa \& Hotta, 1993; Alsheimer \& Benavente, 1996; Alsheimer et al., 2000; Burke \& Stewart, 2002, 2013; Schütz et al., 2005).

On the cytoplasmic side, the ONM is connected to the endoplasmic reticulum (ER). The ONM is characterized by its high content of integral membrane proteins, inter alia, KASH domain proteins (larsicht, $\underline{\mathrm{ANC}}$-1, and $\underline{\mathrm{S} y n e}$ homology) that mediate the linkage of the cytoskeleton and the nucleoskeleton (LINC) via interaction with the conserved SUN domain proteins (Watson, 1955; Burke \& Stewart, 2002; Zhen et al., 2002; Schirmer et al., 2003; Starr, 2003; Starr \& Fridolfsson, 2010).

SUN domain proteins are highly conserved and span the inner nuclear membrane. Their Nterminal region is oriented to the nucleoplasm and interacts with lamins. Their C-terminal region, which contains the SUN domain, interacts with KASH domain proteins in the PNS. Thus, the interaction of SUN-KASH domain proteins forms the LINC complex (Fig. 1.5) (Malone et al., 1999; Burke \& Stewart, 2002; Crisp et al., 2006; Starr \& Fridolfsson, 2010).

The LINC complex is constituted by the association of several proteins, which include not only SUN and KASH proteins (NESPRIN1/2 and Emerin) but also microtubules, actin filaments, lamins A/C, and chromatin (Starr, 2003; Crisp et al., 2006; Tzur et al., 2006; Wilhelmsen et al., 2006). Emerin resides in large part in the INM, whereas a small fraction localizes in the ONM. It is responsible for binding centrosomes to the ONM via microtubules (Salpingidou et al., 2007).

Besides that, centrosome attachment to the nucleus is mediated by the SUN-KASH complex. The centrosomal and nuclear protein ZYG-12 is a member of the HOOK protein family, which are cytosolic coiled-coil proteins, and mediate the binding to organelles and microtubules. ZYG12 has been described in C. elegans and was characterized as a type II ONM protein. ZYG-12 is recruited to the nuclear envelope by SUN1. Thus, ZYG-12 interacts with SUN1 in the PNS, collaborating in the proper junction of the centrosome to the nucleus. Furthermore, zyg-12 gene mutations are harmful by disturbing the nucleus to the centrosome junction, affecting the mitotic spindles and DNA segregation (Malone et al., 2003; Minn et al., 2009). 
Thus, the SUN-KASH protein complex plays an essential role in diverse cellular processes, inter alia, linking nuclear components to the cytoskeleton, mediating chromosome movements in S.pombe, and affecting the migration and location of the nucleus. Further, it mediates the centrosome binding to the nucleus in C. elegans (Malone et al., 2003; Starr \& Han, 2003; Wilhelmsen et al., 2006; Chikashige et al., 2007).

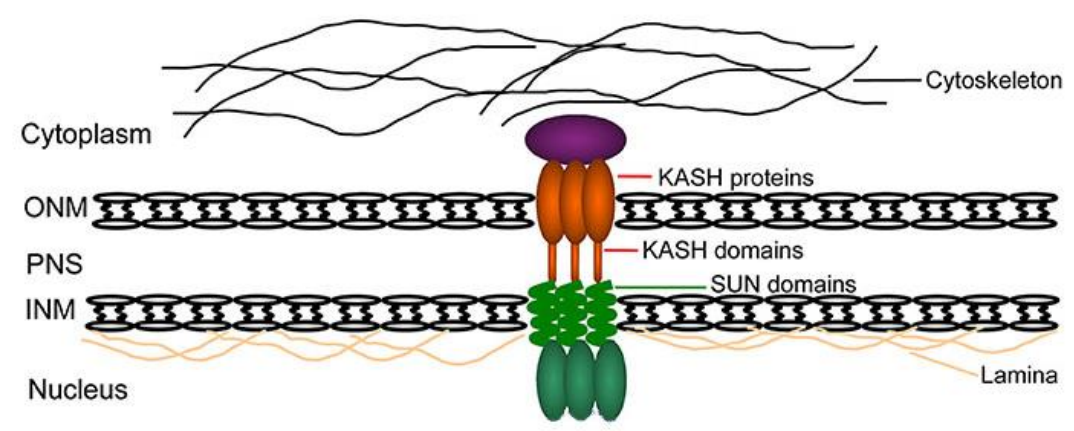

Figure 1.5. Scheme of the LINC complex. The N-terminal region of the SUN domain proteins spans the INM, whereas the C-terminal region containing the SUN domain resides in the perinuclear space (PNS). KASH domain proteins span the ONM and interact with the SUN domain in the PNS, forming the LINC complex that connects the cytoplasm with the nucleoplasm (adapted from Zeng et al., 2018).

\subsection{SUN domain proteins}

SUN domain proteins were first described in Caenorhabditis elegans unc-84, which are involved in nuclear migration. Thereafter, sad-1 was described in $S$. pombe. Hence, the comparison between the C-terminal region of UNC-84, Sad1, and human proteins SUN1 and SUN2 revealed the homology of this region defining the name of SUN domain proteins from

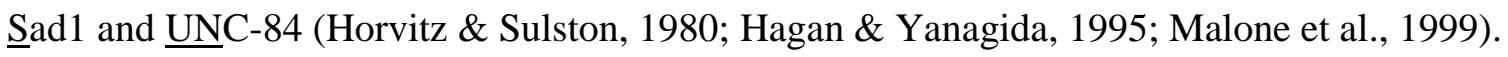

SUN domain proteins share a common structure which consists of an $\mathrm{N}$-terminal region containing up to three transmembrane domains (TMs), a middle part with up to two coiled-coil regions, and a C-terminal region that contains the conserved SUN domain (Crisp et al., 2006; Haque et al., 2006). The $\mathrm{N}$-terminal region extends into the nucleoplasm, whereas the conserved C-terminal of the SUN domain proteins reside in the perinuclear space (Hodzic et al., 2004; Crisp et al., 2006).

The conserved SUN domain proteins play several roles in different organisms, such as spindle architecture, interaction with lamins, telomere anchorage to the nuclear envelope during meiotic 
movements in mice as well as in S. cerevisiae. Furthermore, SUN proteins mediate the centrosomal attachment to the nucleus in C. elegans (Malone et al., 2003; Conrad et al., 2007; Ding et al., 2007; Schmitt et al., 2007).

In mammals, the SUN domain proteins are encoded by a minimum of five genes (Liu et al., 2007). Sun1 and Sun2 are widely expressed in several tissues, and they are crucial in the connection of the cytoskeleton to the nucleoskeleton through giant nesprin and lamins (Hodzic et al., 2004; Padmakumar, 2005; Crisp et al., 2006; Wang et al., 2006). In contrast, SUNC1/SUN3, SPAG4/SUN4, and SPAG4L/SUN5 are expressed predominantly in testes (Göb et al., 2010; Frohnert et al., 2011).

SUN3 is a transmembrane protein with the same topological organization as other SUN domain members. It is expressed in the late stages of spermiogenesis and colocalizes with lamin B3 and lap2 at the posterior pole of spermatids. Moreover, SUN3 overlaps with the manchette in elongating spermatids (Crisp et al., 2006; Göb et al., 2010).

Spag4, also known as Sun4, is spermiogenesis specific and starts its transcription in round spermatids. During spermiogenesis, SPAG4 exhibits an initial expression in round spermatids where it localizes in the nuclear envelope as well as SUN3 and Nesprin1. Afterward, in elongating spermatids, SPAG4 colocalizes with the transient microtubular manchette. In contrast, in the late stages of spermiogenesis, SPAG4 migrates to the distal pole of the sperm nucleus, specifically where the sperm tail is inserted. Moreover, Spag4-deficient mice suffer from severe defects in the manchette structure, chromatin condensation, and sperm head shaping (Calvi et al., 2015; Pasch et al., 2015). Furthermore, Spag4-deficiency affects the correct arrangement and docking of the manchette to the spermatid nucleus, perturbs the HTCA tightness by altering the proper junction of the basal plate to the implantation fossa (Yang et al., 2018). Thus, SPAG4 is part of the complex protein network organization that bridges the nucleus to the sperm tail.

Spag4l, also called Sun5, is highly expressed in testis. The gene SRG4 encodes SUN5 in mouse and its transcription is related to round spermatids (Xing et al., 2004). Spag4l and Spag4l2 are two splice variants of the gene Sun5, which differ in 25 amino acids at the N-terminal region. SPAG4L has one transmembrane domain, whereas the longer isoform, SPAG4L2, contains two transmembrane domains at the N-terminus. Although SUN5 was first described to be associated with the apical nuclear envelope of round spermatids facing the acrosome (Frohnert et al., 2011), later analysis revealed that SUN5 resides at the head-to-tail coupling apparatus in mature spermatozoa (Yassine et al., 2015). Moreover, the partial deletion of Sun5-gene affects the 
HTCA development in mice and causes male infertility due to globozoospermia (Shang et al., 2017).

Furthermore, studies in male patients have identified 12 mutations in the Sun5-gene (Fig. 1.6). Zhu et al. (2016) predicted that Sun5 mutations might cause the acephalic spermatozoa syndrome due to LINC complex alterations. The LINC complex is formed by the interaction of SUN domain proteins with cytoskeletal proteins. Thus, mutations in the SUN domain, as p.Val261Met, p.Thr275Met, p.Ser284, p.Asn348Ile, and p.Arg356Cys, may severely affect their ability to interact with their counterparts. Further, mutations that affect the coiled-coil domain (c.425+1G>A, p.(Leu143Serfs*30), p.Arg159*, p.Met162Lys) and the transmembrane domain (p.Gly114Arg and p.Val128Serfs*7) might disrupt SUN5 localization at the nuclear envelope. Computational prediction and ectopic expression of Sun5 containing the p.Gly114Arg mutation has shown that the SUN5 protein is not restricted to the NE but also distributes to the cytosol. Moreover, p.Gly114Arg mutation inhibits exon 5 splicing and is responsible for the production of acephalic spermatozoa in humans, whereas the frameshift p.(Leu143Serfs*30) deletes 5090 base pairs, which includes exon 8 (Elkhatib et al., 2017). Besides that, the mutation p.Arg $159 *$ (c.475C $>\mathrm{T}$ ) leads to an earlier stop codon that truncates the coiled-coil domain and restrains the transcription of the SUN domain. This mutation is inherited paternally and increases decapitated spermatozoa formation (Shang et al., 2018). (Nomenclature: prefix "p" indicates amino acid change; "c" indicates coding DNA sequence; asterisk indicates translation termination stop-codon)

Among all SUN domain proteins, SUN5 seems to be responsible for the tight linkage of the head to the tail in sperm. Sun5 impairments may disrupt the LINC complex and thereby be responsible for causing the acephalic spermatozoa syndrome.

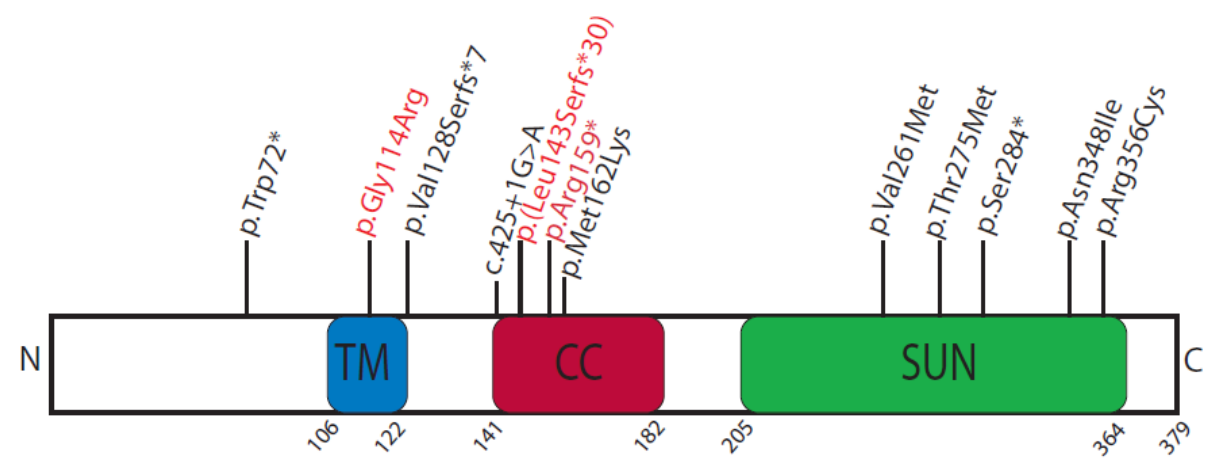

Figure. 1.6. Schematic localization of human SUN5 mutations. Transmembrane domain (TM) (blue), coiled-coil domain (red), and SUN domain (green) (adapted from Zhu et al., 2016; Shang et al., 2018). 


\subsection{Male infertility due to acephalic spermatozoa syndrome and its genetic etiology}

Several morphological abnormalities of the spermatozoa could cause male infertility. These abnormalities include sperm flagella disorders, impairment in head-neck attachment, irregular acrosome development, and chromatin condensation (Chemes \& Rawe, 2003).

In the present work, I focused my attention on abnormalities of the HTCA caused by several defects in the connecting piece and centrioles formation. These aberrations produce acephalic or pinhead spermatozoa, a type of human teratozoospermia (TZI) (WHO, 2010; Chemes \& Alvarez Sedo, 2012). HTCA abnormalities lead to a fragile head-tail junction or may severely cause spermatozoa decapitation. Ultra-structural analyses have indicated that the instability of the linkage of the connecting piece to the sperm nucleus caused abnormal alignment of the sperm head to the midpiece. This disorder includes an irregular formation of the basal plate, implantation fossa, and the inability of the proximal centriole to migrate to the posterior area of the sperm nucleus. The proximal centriole attaches to the spermatid nucleus. However, its dysfunction affects its physical contact with the nucleus and causes the separation of sperm head and tail (Chemes et al., 1999; Chemes \& Rawe, 2010; Yang et al., 2018). Thus, centriolar and connecting piece dysfunctions seem to have a genetic etiology in humans (Baccetti et al., 1989; Chemes et al., 1999). Yet, molecular components of the HTCA have been scarcely characterized. In humans, solely the gene Sun 5 has been identified to be responsible for the acephalic spermatozoa syndrome. However, in mice models, several genes have been reported to be involved in the head-tail junction, inter alia, Prss21, Oaz3, Cntrob, Ift88, Spata6, Odf1, Hook1, Arl3, RIM-BP3, and Sun5 (Mendoza-Lujambio, 2002; Netzel-Arnett et al., 2009; Tokuhiro et al., 2009; Zhou et al., 2009; Liška et al., 2013; Qi et al., 2013; Yuan et al., 2015; Zhu et al., 2016).

In mice, the proteins Centrobin (encoded by Cntrob), Ift88, RIMBP3, and HOOK1 localize in the manchette, and their disruption affects spermatid elongation. Centrobin resides at the marginal ring of the spermatid acroplaxome, manchette, and HTCA. In mice, the disruption of Cntrob affects the manchette assembly and the intramanchette transport, which is responsible for transporting proteins to the HTCA and tail formation. Thus, Centrobin depletion affects the attachment of the HTCA and implantation fossa contributing to sperm decapitation (Liška et al., 2009). Likewise, the intra-flagellar transport protein IFT88 is involved in the formation of the acrosome-acroplaxome complex, HTCA, and the spermatid tail. Ift88-disruption affects the intramanchette transport inhibiting the tail development in spermatids (Kierszenbaum et al., 2011). 
RIM-BP3 localizes in the manchette of developing spermatids and the gene disruption severely affects the acrosome, acroplaxome, manchette, and nucleus. Moreover, RIM-BP3 interacts with HOOK1, which resides in the manchette (Zhou et al., 2009). HOOK1 belongs to the conserved HOOK protein family, which has a highly conserved $\mathrm{NH}_{2}$ domain, a coiled-coil domain in the middle part, and a C-terminal domain. These domains are responsible for binding microtubules, promoting the interaction with other proteins, and binding organelles. Moreover, HOOK1 mediates the manchette connection to the nuclear envelope through its $\mathrm{C}$-terminal region. Thus, the truncation of HOOK1 in mice causes abnormal spermatozoon head shape (azh) and male infertility (Walenta et al., 2001; Mendoza-Lujambio, 2002).

The proteins PRSS21, OAZ3, SPAT6, and ODF1 contribute to the connection of the head to the tail in sperm and their disruption is related either with a weak connecting piece or total sperm decapitation. Mice lacking PRSS21 (Glycosylphosphatidylinositol-Anchored Serine Protease) demonstrate spermatozoa with abnormal curled tails, decreased motility, abnormal heads and tails orientation, and fragile neck. Thus, during the passage through the epididymis, spermatozoa suffer from decapitation (Netzel-Arnett et al., 2009). Similarly, Oaz3-null male mice (Ornithine decarboxylase antizymes) are infertile. They exhibit easy separation of heads from tails due to basal plate and capitulum separation (Tokuhiro et al., 2009). SPATA6 resides exclusively on the connecting piece and Spata6-gene disruption in mice disarranges the basal plate connection to the implantation fossa. Thus, Spata6 mutant mice cause male infertility due to acephalic spermatozoa, which are devoid of the connecting piece, segmented columns, and capitulum (Yuan et al., 2015). ODF1 is a cytoskeletal protein situated in the basal plate, and the ablation of the Odfl-gene produces sperm decapitation due to an impairment in the sperm headto-tail linkage (Yang et al., 2012).

In mice, the disruption of Sun5 causes male infertility affecting the head-tail coupling apparatus, whereas, in humans, 12 mutations of the gene have been identified. Thus, specific mutations in Sun5 truncate the translation of the protein affecting its localization at the nuclear envelope. These genetic disorders prevent the centriole attachment to the nucleus, disrupt the HTCA, are responsible for the acephalic spermatozoa syndrome, and cause male infertility (Elkhatib et al., 2017; Shang et al., 2018). The SUN5 protein is described in more detail in chapter 1.6. Thus, identifying the molecular components involved in the acephalic spermatozoa syndrome may contribute to a better understanding of male germ cell differentiation and design diagnostics and treatments in assisted reproduction technology. 


\subsection{Research objectives}

ODF1 is an essential component of the HTCA, and its disruption causes sperm decapitation (Yang et al., 2012; 2014). Even though ODF1 is involved in the linkage of the head to the tail in sperm, its interacting partners that mediates this linkage are still unknown. Besides that, Odfldepletion, the partial disruption of Spag4l and Hookl cause male infertility in mice, suggesting that the acephalic spermatozoa (AS) syndrome has a genetic origin, albeit few mutations have been identified in humans. Given that ODF1 mediate the attachment of the connecting piece to the sperm nucleus and mutations in this gene have not been reported, I explored the OdfIsequence in two patients who exhibited the AS-phenotype. Mutation screening also included regions of the genes Spag4l and Hookl.

ODF1 interacts with the SUN domain protein SPAG4 (Shao et al., 1999), although this interaction is unusual for SUN-domain proteins. SUN proteins localize at the nuclear envelope (NE), whereas ODF1 localizes in the cytoskeletal area of the HTCA. Hence, this study focused on characterizing the topology of SUN4 and SUN5 to obtain more insights about their orientation in the NE. Thus, I evaluated the topology of Spag4l domains by immunocytology. Due to the localization of the SUN-domain proteins at the NE, Spag4-depletion might disturb gene transcription during spermatogenesis. Therefore, I evaluated marker genes during spermatogenesis progression in Spag $4^{-/-}$mice.

In order to identify additional interactors of SPAG4 apart from ODF1, I explored the amber suppression method, which is based on incorporating a photoreactive unnatural amino acid as a coding codon into a protein. This protein, after photo-activation, cross-links with nearby proteins that afterward can be identified by Western blot. Further, to obtain more insights into the ability of SPAG4 to interact with ODF1, I investigated the orientation of SPAG4 and SPAG4L at the nuclear membrane. For this purpose, recombinant proteins were induced to dimerize by adding the immunosuppressant "rapamycin." To further analyze the function of SPAG4L regarding male fertility, a part of this project was centered on the generation of Spag4ldeficient mice.

The last objective was to identify new ODF1 interacting partners that collaborate in the HTCA formation. The focus was centered on CCDC42 due to its reported effect on the formation of the HTCA in mice (Pasek et al., 2016). Besides that, the expression and localization of CFAP52 were analyzed because of its physiological functions, potential role in axoneme stability, and its ability to mediate protein interactions (Li et al., 2001). 


\section{Material and methods}

\subsection{Material}

\subsubsection{Biological material}

\subsubsection{Bacterial strains}

Competent Escherichia coli strain DH5 $\alpha$ and D $\alpha 10 \mathrm{~b}$ were used to subclone plasmid DNA.

\subsubsection{Cell lines}

Two different cell lines were cultivated. The cell line derived from mouse embryonic fibroblast (NIH3T3) (Todaro \& Green, 1963) and cells derived from human embryonic kidney (HEK293) (Graham et al., 1977).

\subsubsection{Vectors}

Different vectors were used either for cloning or protein expression in eukaryotic cells. The vectors $\mathrm{pCR}^{\mathrm{TM}} \mathrm{II}$ and $\mathrm{pJET} 1.2$ were used for cloning, whereas pcDNA3.1 (-) C (Invitrogen) and pEGFP-C1 (Clontech) were used for overexpression in mammalian cells.

\subsubsection{Oligonucleotides}

Oligonucleotides were designed by NCBI/primer BLAST, whereas primer properties were $\begin{array}{llll}\text { evaluated } & \text { using } & \text { PCR }\end{array}$ (https://www.bioinformatics.org/sms2/pcr_primer_stats.html). Oligonucleotide primers were ordered either from Integrated DNA technologies IDT or Eurofins and dissolved in distilled water to a final concentration of $100 \mu \mathrm{M} / \mu \mathrm{l}$, according to the manufacturer. Oligonucleotides are listed in 6.1 . 


\subsubsection{Enzymes}

\begin{tabular}{|c|c|c|}
\hline Enzyme Type & Enzyme & Resource \\
\hline \multirow{13}{*}{ Restriction Enzyme } & AgeI-HF & New England Biolabs \\
\hline & BamHI-HF & New England Biolabs \\
\hline & BglII & New England Biolabs \\
\hline & EcoRI-HF & New England Biolabs \\
\hline & EcoRV-HF & New England Biolabs \\
\hline & HindIII-HF & New England Biolabs \\
\hline & KpnI-HF & New England Biolabs \\
\hline & NheI-HF & New England Biolabs \\
\hline & NotI-HF & New England Biolabs \\
\hline & SacI-HF & New England Biolabs \\
\hline & SpeI-HF & New England Biolabs \\
\hline & SpeI-HF & New England Biolabs \\
\hline & XbaI & New England Biolabs \\
\hline \multirow{4}{*}{ DNA-polymerase } & Taq polymerase & Fermentas \\
\hline & MangoTaq $^{\text {TM }}$ DNA Polymerase & Bioline \\
\hline & Phusion & Own synthesis \\
\hline & Advantage 2 Polymerase Mix & Clontech \\
\hline \multirow{3}{*}{$\begin{array}{l}\text { DNA polymerase mix for } \\
\text { qPCR }\end{array}$} & 2X KAPA SYBR® FAST qPCR & KAPA Biosystem \\
\hline & All-in-One ${ }^{\mathrm{TM}} \mathrm{qPCR}$ & GeneCopoeia \\
\hline & $\begin{array}{c}\text { HOT FIREpol }{ }^{\circledR} \text { EvaGreen }{ }^{\circledR} \text { qPCR Mix } \\
\text { Plus (ROX) }\end{array}$ & Solis BioDyne, Tartu, Estland \\
\hline Klenow fragment & DNA Polymerase I & Thermo Scientific \\
\hline DNA ligase & T4 DNA Ligase & New England Biolabs \\
\hline Proteinase $\mathrm{K}$ & Proteinase $\mathrm{K}$ & Roth \\
\hline
\end{tabular}

Table 2.1. Enzymes 


\subsubsection{Antibodies}

\subsubsection{Primary antibodies}

\begin{tabular}{|c|c|c|c|c|}
\hline Antibody & Target protein & $\begin{array}{c}\text { Host } \\
\text { animal }\end{array}$ & Code & Reference \\
\hline AntiRedFP & mCherry & rat & $5 \mathrm{~F} 8$ & Chromotek \\
\hline \multirow[t]{2}{*}{ Anti-GFP } & pEGFP & rabbit & An\#00002 & Own synthesis \\
\hline & ECFP & & & First bleeding \\
\hline \multirow{2}{*}{ Anti-GFP } & $\mathrm{pEGFP}$ & mouse & & Own synthesis \\
\hline & ECFP & & & Ibrahim Adham \\
\hline \multirow[t]{5}{*}{ Anti-CCDC42 } & COILED-COIL & rabbit & Bs-8131R & Antibodies \\
\hline & DOMAIN & & ABIN872760 & Online \\
\hline & CONTAINING 42 & & & \\
\hline & $(\mathrm{CCDC} 42)$ & & & \\
\hline & Epitope KHL & & & \\
\hline \multirow[t]{5}{*}{ Anti- CCDC42 } & COILED-COIL & rabbit & ARP52735_P050 & Antibodies \\
\hline & DOMAIN & & ABIN2785068 & Online \\
\hline & CONTAINING 42 & & & \\
\hline & $(\mathrm{CCDC} 42)$ & & & \\
\hline & Epitope middle region & & & \\
\hline Anti-ODF1 & $\begin{array}{l}\text { Outer dense fiber of } \\
\text { sperm tails } 1 \text { (ODF1) }\end{array}$ & rabbit & & $\begin{array}{l}\text { Antibodies } \\
\text { online }\end{array}$ \\
\hline Anti-Pericentrin & Pericentrin & rabbit & PRB432C & Covance \\
\hline Anti-gamma Tubulin & $\gamma$-tubulin & mouse & GTU-88 & Sigma-Aldrich \\
\hline \multirow[t]{2}{*}{ Anti-myc } & Myc-tag & rabbit & AA31-80 & Antibodies Online \\
\hline & & & ABIN1532205 & \\
\hline Anti-Myc polyclonal & Myc-tag & rabbit & & Millipore \\
\hline Anti-HA & HA-tag & mouse & $\mathrm{C} 5$ & Abcam \\
\hline Anti-CFAP52 & CFAP52 & rabbit & PA839781LA01 & Cusabio \\
\hline
\end{tabular}

Table 2.2. Primary antibodies 


\subsubsection{Secondary antibodies}

\begin{tabular}{ccccc}
\hline $\begin{array}{c}\text { Antibody } \\
\text { goat anti-mouse HRP- } \\
\text { coupled }\end{array}$ & $\begin{array}{c}\text { Target } \\
\text { protein }\end{array}$ & $\begin{array}{c}\text { Host } \\
\text { animal }\end{array}$ & Code & Reference \\
\hline $\begin{array}{c}\text { mouse-HRP } \\
\text { coupled }\end{array}$ & goat & & $\begin{array}{c}\text { Jackson Immuno } \\
\text { Research }\end{array}$ \\
\hline goat anti-rat HRP-coupled & Rat-HPR & goat & & $\begin{array}{c}\text { Jackson Immuno } \\
\text { Research }\end{array}$ \\
\hline goat anti rabbit-IgG (H+L) & rabbit IgG & goat & Alexa fluor R 555 & Life Technologies \\
\hline goat anti-mouse-IgG (H+L) & mouse IgG & goat & Alexa fluor R 555 & Molecular probes \\
\hline goat $\alpha$ guinea pig-IgG & guinea pig & goat & Cy3 & Dianova \\
\hline goat $\alpha$ mouse & IgG & & & Moat \\
\hline goat anti-rabbit & rabbit IgG & goat & MFP488 & Mobitec \\
\hline goat $\alpha$ rabbit IgG & rabbit IgG & goat & DyLight 488 & Thermo Scientific \\
\hline goat $\alpha$ mouse IgG & mouse IgG & goat & DyLight 488 & Thermo Scientific
\end{tabular}

Table 2.3. Secondary antibodies 


\subsubsection{Culture media}

\subsubsection{Culture media for eukaryotic cells}

\begin{tabular}{|l|l|}
\hline \multicolumn{1}{|c|}{ Cell line } & \multicolumn{1}{c|}{ Ingredients } \\
\hline NIH3T3 & $\begin{array}{l}\text { Gibco DMEM + Glutamax-I 1x 500 ml } \\
\text { (contains 4.5 g/l D-glucose and sodium } \\
\text { HEK293 }\end{array}$ \\
& \\
\hline
\end{tabular}

Table 2.4. Culture media for eukaryotic cells

\subsubsection{Culture media for prokaryotic cells}

\begin{tabular}{|l|l|}
\hline LB-Medium & $10 \mathrm{~g}$ Trypton \\
& $10 \mathrm{~g} \mathrm{NaCl}$ \\
& $\begin{array}{l}5 \mathrm{~g} \text { yeast extract } \\
* \text { Ampicillin at } 100 \mu \mathrm{g} / \mathrm{mL} \text { or Kanamycin at } 50 \\
\mathrm{mg} / \mathrm{mL}\end{array}$ \\
\hline LB agar plate & $1.5 \%(\mathrm{w} / \mathrm{v})$ agar in LB medium \\
\hline
\end{tabular}

Table 2.5. Culture media for prokaryotic cell culture

\subsubsection{Buffers and solutions}

\begin{tabular}{|l|l|}
\hline \multicolumn{1}{|c|}{ Buffer/solution name } & \multicolumn{1}{c|}{ Ingredients } \\
\hline Blocking solution (Western-Blot) & $\begin{array}{l}\text { 5\%(w/v) skimmed milk powder } \\
\text { diluted in 1X-TBST buffer }\end{array}$ \\
\hline Blocking solution (Immunocytology)) & $1 \%(\mathrm{w} / \mathrm{v}) \mathrm{BSA}$ in PBS \\
& $0.5 \%(\mathrm{v} / \mathrm{v})$ Tween \\
diluted in 1X-PBS buffer
\end{tabular}




\begin{tabular}{|c|c|}
\hline & $\begin{array}{l}2 \%(\mathrm{w} / \mathrm{v}) \text { SDS } \\
10 \%(\mathrm{w} / \mathrm{v}) 2-\beta \text {-Mecaptoethanol } \\
0.001 \%(\mathrm{w} / \mathrm{v}) \text { bromophenol blue } \\
10 \%(\mathrm{w} / \mathrm{v}) \text { glycerol }\end{array}$ \\
\hline DNA loading buffer $6 \mathrm{X}$ & $\begin{array}{l}0.09 \%(\mathrm{w} / \mathrm{v}) \text { xylene cyanol FF } \\
60 \mathrm{mM} \text { EDTA } \\
0.09 \%(\mathrm{w} / \mathrm{v}) \text { bromophenol blue } \\
60 \%(\mathrm{w} / \mathrm{v}) \text { glycine }\end{array}$ \\
\hline KHM buffer & $\begin{array}{l}110 \mathrm{mM} \text { KOAc } \\
20 \mathrm{mM} \text { Hepes, pH } 7.4 \\
2 \mathrm{mM} \mathrm{MgCl}_{2}\end{array}$ \\
\hline Laemmli electrophoreses buffer (10X) & $\begin{array}{l}0.25 \mathrm{M} \text { Tris } \\
2 \mathrm{M}(\mathrm{w} / \mathrm{v}) \text { glycine } \\
1 \%(\mathrm{w} / \mathrm{v}) \mathrm{SDS}\end{array}$ \\
\hline Lysis buffer for eukaryotic cells & $\begin{array}{l}150 \mathrm{mM} \mathrm{NaCl} \\
2 \mathrm{mM} \text { EDTA } \\
1 \% \text { Triton X-100 } \\
1 \mathrm{X} \text { of protease inhibitors cocktail 100X } \\
\text { In PBS (pH 7.2) }\end{array}$ \\
\hline Lysis buffer for prokaryotic cells & $\begin{array}{l}150 \mathrm{mM} \mathrm{NaCl} \\
250 \mathrm{mM} \text { Tris-HCl }(\mathrm{pH} 7.2) \\
0.1 \% \text { Triton X-100 } \\
1 \mathrm{mM} \text { DTT } \\
1 \mathrm{mM} \text { PMSF }\end{array}$ \\
\hline Lysis buffer for in situ proteinase $\mathrm{K}$ digestion & $\begin{array}{l}0.4 \% \text { SDS } \\
2 \% \text { Triton X-100 } \\
400 \mathrm{mM} \mathrm{NaCl} \\
50 \mathrm{mM} \text { Tris-HCl, } \mathrm{pH} 7.4 \\
1 \mathrm{mM} \text { DTT } \\
2 \mu \mathrm{g} / \mathrm{ml} \text { pepstatin A } \\
1 \mu \mathrm{g} / \mathrm{ml} \text { Leupeptin }\end{array}$ \\
\hline NID buffer & $\begin{array}{l}50 \mathrm{mM} \mathrm{KCl} \\
10 \mathrm{mM} \text { Tris- } \mathrm{HCl}(\mathrm{pH} 8,3) \\
2 \mathrm{mM} \mathrm{MgCl} 2 \\
0.1 \mathrm{mg} / \mathrm{ml} \text { gelatine } \\
0.45 \% \text { (v/v) NP40 } \\
0.45 \%(\mathrm{v} / \mathrm{v}) \text { Tween } 20 \\
\text { Proteinase K 1,6U/300 } \mu \mathrm{l}\end{array}$ \\
\hline
\end{tabular}




\begin{tabular}{|c|c|}
\hline Paraformaldehyde $3.7 \%$ & $\begin{array}{l}3.7 \text { gr Paraformaldehyde } \\
\text { diluted in } 1 \mathrm{X}-\mathrm{PBS}\end{array}$ \\
\hline PBS buffer (10X) & $\begin{array}{l}140 \mathrm{mM} \mathrm{NaCl} \\
7 \mathrm{mM} \mathrm{Na}_{2} \mathrm{HPO}_{4} \\
3 \mathrm{mM} \mathrm{KH}_{2} \mathrm{PO}_{4}\end{array}$ \\
\hline RIPA buffer & $\begin{array}{l}150 \mathrm{mM} \mathrm{NaCl} \\
1 \% \text { Nonidet P40 } \\
0.5 \% \text { Sodium Deoxycholate } \\
0.1 \% \text { SDS } \\
50 \mathrm{mM} \text { Tris (pH 7.7) } \\
10 \mathrm{mM} \text { PMSF }\end{array}$ \\
\hline 10X RIPA buffer for crosslinking assay & $\begin{array}{l}0.5 \mathrm{M} \text { Tris- } \mathrm{HCl}(\mathrm{pH} 7.4) \\
1.5 \mathrm{M} \mathrm{NaCl} \\
2.5 \% \text { Deoxycholate } \\
10 \% \mathrm{NP}-40 \\
10 \mathrm{mM} \text { EDTA }\end{array}$ \\
\hline Stripping solution & $\begin{array}{l}1.5 \%(w / v) \text { glycine } \\
0.1 \%(w / v) \text { SDS } \\
1 \% \text { Tween } 20 \\
\text { Final pH } 2.2\end{array}$ \\
\hline TAE buffer (50X) & $\begin{array}{l}242 \mathrm{~g} / \mathrm{l} \text { Tris } \\
57.1 \mathrm{ml} / \mathrm{l} \text { glacial acetic acid } \\
100 \mathrm{ml} \text { of } 0.5 \mathrm{M} \text { EDTA } \\
\text { Final pH } 8.0\end{array}$ \\
\hline TBST & $\begin{array}{l}20 \mathrm{mM} \text { Tris- } \mathrm{HCl}(\mathrm{pH} 7.5) \\
300 \mathrm{mM} \mathrm{NaCl} \\
0.2 \% \text { Triton } \mathrm{X}-100\end{array}$ \\
\hline Transfer buffer & $\begin{array}{l}1 \mathrm{M} \text { Tris- } \mathrm{HCl}(\mathrm{pH} 7.5) \\
20 \mathrm{M} \text { glycine } \\
0.4 \mathrm{mM} \mathrm{MgCl}_{2} \\
0.04 \% \text { (w/v) SDS } \\
20 \% \text { methanol }\end{array}$ \\
\hline
\end{tabular}

Table 2.6. Buffers and solutions 


\subsubsection{Chemicals}

\begin{tabular}{|c|c|}
\hline Name & Company \\
\hline 2- $\beta$ - Mercaptoetanol & Sigma-Aldrich \\
\hline Agarose & Serva \\
\hline Ampicillin & Serva \\
\hline APS & Sigma-Aldrich \\
\hline Bromophenol blue & Sigma-Aldrich \\
\hline BSA & AppliChem \\
\hline $\mathrm{CaCl}_{2}$ & Merk \\
\hline DAPI & Sigma-Aldrich \\
\hline DTT & Sigma-Aldrich \\
\hline EDTA & Roth \\
\hline Ethanol & VWR Chemicals \\
\hline Fluoromount-G $\mathrm{G}^{\mathrm{TM}}$ & Invitrogen \\
\hline Glacial acetic acid & ChemSolute \\
\hline Glucose & Roth \\
\hline Glycerol & Roth \\
\hline Glycine & Roth \\
\hline $\mathrm{HCl}(37 \%)$ & Roth \\
\hline HEPES & Roth \\
\hline Imidazole & Roth \\
\hline IPTG & Sigma-Aldrich \\
\hline Kanamycin & Sigma-Aldrich \\
\hline $\mathrm{KCl}$ & Merk \\
\hline $\mathrm{KH}_{2} \mathrm{PO}_{4}$ & Merk \\
\hline KOAc & Roth \\
\hline Methanol & VWR Chemicals \\
\hline $\mathrm{MgCl}_{2}$ & AppliChem \\
\hline $\mathrm{MgSO}_{4}$ & AppliChem \\
\hline $\mathrm{Na}_{2} \mathrm{HPO}_{4}$ & Merk \\
\hline $\mathrm{NaCl}$ & AppliChem \\
\hline $\mathrm{NaHCO}_{3}$ & Sigma-Aldrich \\
\hline Nonidet P40 & Fluka \\
\hline PFA & Sigma-Aldrich \\
\hline
\end{tabular}




\begin{tabular}{|l|l|}
\hline Pierce Protein G Agarose & Thermo Fisher Scientific \\
\hline PL-FITC & PL biochemicals \\
\hline PMSF & Sigma-Aldrich \\
\hline PageRuler ${ }^{\text {TM } \text { Prestained Protein Ladder \#26616 }}$ & Thermo Scientific \\
\hline $\begin{array}{l}\text { Protease inhibitors } \\
\text { cOmplete Ultra tabler mini EDTA-free }\end{array}$ & Roche \\
\hline 100X Protease inhibitor cocktail & Cell signaling \\
\hline Rotiphorese gel 40 (29:1) & Roth \\
\hline Rapamycin & Millipore \\
\hline SDS & Roth \\
\hline Serva DNA Stain G & Serva \\
\hline Skimmed milk powder (slightly soluble) & Difco \\
\hline Sodium acetate & AppliChem \\
\hline S.O.C medium & Invitrogen \\
\hline TEMED & Roth \\
\hline Tris & Roth \\
\hline Triton X-100 & Roth \\
\hline Trypsin-EDTA 0,25\% & Life technologies \\
\hline Tween 20 & Roth \\
\hline X-gal & Thermo Fisher Scientific \\
\hline
\end{tabular}

Table 2.7. Chemicals 


\subsubsection{Kits}

\begin{tabular}{|c|c|}
\hline Ambion® Acid Phenol:Chloroform: IAA & Thermo Fisher Scientific \\
\hline Antarctic Phosphatase & BioLabs \\
\hline Clarity Max ${ }^{\text {TM }}$ Western ECL Substrate & Bio-Rad \\
\hline Dual promoter TA Cloning ${ }^{\mathrm{TM}} \mathrm{Kit}$ & Invitrogen \\
\hline EndoFectin Max transfection reagent & Genecopoeia \\
\hline KAPA $^{\text {TM} M o u s e ~ G e n o t y p i n g ~ H o t ~ S t a r t ~ K i t ~}$ & KAPA Biosystem \\
\hline $\begin{array}{l}\text { Maxima first-strand cDNA Synthesis Kit for RT- } \\
\text { qPCR }\end{array}$ & Thermo Fisher Scientific \\
\hline 5X MEGAscript ${ }^{\circledR}$ T7 kit & Invitrogen by Thermo Fisher Scientific \\
\hline Miniprep kit NucleoSpin $®$ plasmid & Macherey-Nagel GmbH\&CoKG \\
\hline NucleoSpin ${ }^{\circledR}$ Gel and PCR Clean-Up & Macherey-Nagel GmbH\&CoKG \\
\hline peqGold RNA Pure & PEQLAB \\
\hline Phenol:Chloroform:IAA, 25:24:1 & Ambion \\
\hline Plasmid Midi Kit & QIAGEN \\
\hline Protein G-Agarose & Thermo Fisher Scientific \\
\hline Qubit $^{\mathrm{TM}}$ dsDNA HS Assay Kit & Invitrogen \\
\hline Qubit $^{\mathrm{TM}}$ RNA HS Assay Kit & Invitrogen \\
\hline TA Cloning ${ }^{\mathrm{TM}}$ Kit, Dual Promoter & Invitrogen \\
\hline TURBO DNA-free ${ }^{\text {TM }}$ Kit & Life technologies \\
\hline Viagen DirectPCR-Tail & Viagen Biotech \\
\hline
\end{tabular}

Table 2.8. Kits

\subsubsection{Software and web services}

\begin{tabular}{|l|l|}
\hline \multicolumn{1}{|c|}{ Software or web service } & \multicolumn{1}{c|}{ Website } \\
\hline ZEN blue and black & Microscope Software ZEN from ZEISS Microscopy \\
\hline Adobe Photoshop 7.0 & \\
\hline NCBI & https://www.ncbi.nlm.nih.gov/ \\
\hline ENSEMBL & https://www.ensembl.org/index.html \\
\hline BLAST & https://blast.ncbi.nlm.nih.gov/Blast.cgi \\
\hline Expasy & https://web.expasy.org/translate/ \\
\hline SAC multalin & http://multalin.toulouse.inra.fr/ \\
\hline Clustal Omega & https://www.ebi.ac.uk/Tools/msa/clustalo/ \\
\hline
\end{tabular}

Table 2.9. Software and services 


\subsection{Methods}

\subsubsection{Microbiological methods}

\subsubsection{Liquid culture of E.coli}

Transformed bacteria from a colony or a glycerol stock were cultivated in LB medium ( $5 \mathrm{ml}$ to $100 \mathrm{ml}$ ) containing selective antibiotics, either ampicillin or kanamycin (at either $100 \mu \mathrm{g} / \mathrm{mL}$ or $50 \mathrm{mg} / \mathrm{mL}$, respectively). Bacteria were shaken at $225 \mathrm{rpm}$ at $37^{\circ} \mathrm{C}$ overnight. Bacterial cultures containing a specific plasmid were used to prepare glycerol stocks (2.2.1.2) or purify DNA plasmid (2.2.2.4).

\subsubsection{Storage of transformed bacteria (E. coli)}

For long-term storage of bacterial transformants, glycerol stocks were prepared. Bacteria were grown overnight in media containing the selective antibiotic. Then $800 \mu \mathrm{l}$ of liquid culture was gently mixed by pipetting with $200 \mu$ l of sterile glycerol and stored at $-80^{\circ} \mathrm{C}$.

\subsubsection{Transformation}

$10-50 \mu 1$ of competent bacteria cells, either DH5 $\alpha$ or DH10b, were defrosted on ice and mixed with $20 \mu 1$ of ligation reaction (2.2.2.8). The mixture was incubated on ice for 30 minutes. The cells were then heat-shocked for 90 seconds at $42^{\circ} \mathrm{C}$ and chilled for 2 minutes on ice. Afterward, $200 \mu 1$ of SOC medium was added to the DNA-competent bacteria mixture and incubated for 1 hour at $37^{\circ} \mathrm{C}$. Finally, the transformation mix was plated on LB agar plates containing the appropriate selective antibiotic and incubated-overnight at $37^{\circ} \mathrm{C}$.

\subsubsection{Molecular biological methods}

\subsubsection{Plasmid DNA preparation from E.coli}

Plasmid DNA was prepared in three quantitative scales. For small-scale plasmid preparation, the-Mini Kit NucleoSpin ${ }^{\circledR}$ Plasmid was used (Tab. 2.8). To obtain a large-scale DNA preparation, either Midiprep Kit NucleoSpin ${ }^{\circledR}$ Plasmid or Maxiprep was used. The procedure was according to the manufacturer's instruction manual. 


\subsubsection{Plasmid construction}

\subsection{Subcloning to validate the amber suppression method}

First, LaminC2 and Lamin B3 were amplified from mice testes cDNA by RT-PCR (Tab. 6.1.4). PCR was performed using Phusion polymerase. Amplified fragments were purified from the agarose gel (2.2.2.4) and subsequently subcloned into the vector pJET1.2-blunt (Fermentas). LaminC2 and LaminB3 were excised from vector pJet1.2 using XbaI and KpnI restriction enzymes. After that, each fragment was subcloned into the expression vector pcDNA3.1 (Invitrogen). The successful cloning was verified via sequencing (2.2.2.9).

The LaminC coding sequence was subcloned into pEGFP-N1. The LaminC coding sequence was site-direct mutated by introducing the Amber stop codon (TAG) at three different positions encoding either the amino acid arginine in position 470 (R470), threonine in position 488 (T488), or threonine in position 534 (T534). The complete constructs containing the sitedirected mutations denominated R470, T488, T534, and the aminoacyl-tRNA were kindly provided by Dr. Petra Neumann-Staubitz.

\subsection{Subcloning of SUN domain proteins}

Sun1 and Sun3 were amplified from mice testes cDNA (Table 14). PCR was performed using Phusion polymerase. Amplified fragments were purified from the agarose gel and subsequently subcloned into the vector pJET1.2-blunt (Fermentas). Sunl was cut out from the pJET1.2 vector using NheI and BamHI, whereas Sun3 was digested with restriction enzymes NheI and KpnI (2.2.2.6). Both linearized fragments were subcloned into Cherry-pCR3.1. Sequences of expression plasmids were verified via sequencing (2.2.2.9).

The full-length Spag4l2 was amplified from mouse testes cDNA as previously described (Frohnert et al., 2010) and used as a template for PCR. Spag4l2 contains two transmembrane domains denominated TM1 (TM38-58) and TM2 (TM77-95).

The TM1 (TM 38-58) was first amplified using the primer pair primer1 and primer TM38-58r. The amplified fragment was cloned into pcDNA3.1 myc/His (-) C (Invitrogen, Paisley, UK). Then, the TM1 insert was excised with NheI-EcoRI from the vector pcDNA3.1 myc/His (-) C. The TM1 insert was subcloned into pEGFP-N1 (Clontech, Palo Alto, CA, USA).

The TM2 (TM77-95) was amplified using the primers: primer1, primer2, primer3 TM2, and Spag77-95r EcoRI. The PCR product was digested with NheI-EcoRI and cloned into pEGFPN1 (Clontech, Palo Alto, CA, USA). 
The TM1-TM2 was amplified using the primer pair primer1 and Spag77-95r EcoRI and cloned into pcDNA3.1 myc/His (-) C. The N-terminal region containing the HA tag, TM1 and TM2 were digested with NheI and EcoRI. The digested fragment was cloned into pEGFP-N1 (Clontech, Palo Alto, CA, USA).

The full-length Spag4l2 sequence was amplified using the primers pair primer1 and primer Spag77-95r EcoRI. Afterward, the fragment was cloned into pCDNA3.1 myc/His (-) C (Invitrogen, Paisley, UK).

The expression plasmid HA-Spag4l2-myc containing a frameshift mutation at its C-terminus was generated by PCR using the primers pair Spag4XbaI-for and Spag4KpnI-frameshift. The PCR product was cloned into pCRII and then digested with XbaI and KpnI. Finally, the fragment was subcloned in pcDNA3.1 myc/His (-) C.

Spag4Lc-EGFP was generated by PCR amplification using the primer pair Spag4lC-term-FHindIII and Spag4l C-term-rev-EcoRI. Then, the amplified fragment was subcloned into pEGFP-N1. Finally, all clones were verified by DNA sequencing.

\subsection{Subcloning of genes for the rapamycin system}

The expression plasmid $F R B$-ECFP-Spag4 was prepared by amplifying the fragment $m E C F P$ $F R B$ from the construct $m E C F P-F R B$-in pCRII (Ref: Hoyer-Fender). The primer pair used were ECFP-N-NheI and FIASH-C-NheI (Table 6.1.6). The amplified fragment was subcloned into pCRII. Then, the $p C R I I-m E C F P-F R B$ plasmid was digested with NheI, whereas HA-Spag4-myc in pcDNA3.1 (Ref: W. Sura) was linearized with NheI and XbaI. Finally, both fragments were ligated.

The plasmid Luciferase-mRFP-FKBP was prepared by amplifying the Luciferase gene from pGL-3 (Promega) with primers Luc-NheI-For2 and Luc-AgeI-Rev2 (Tab. 6.1.5). The amplified Luciferase fragment was subcloned into pJET1.2 blunt vector (Fermentas). Subsequently, the plasmid was digested with restriction enzymes NheI (at the 5'-end) and AgeI (at the $3{ }^{\text {' }}$-end). The expression plasmid $m R F P-F K B P$ in pEGFP-N1 (Ref: C. Schultz/M. Schifferer) was linearized with the restriction enzyme AgeI (at the 5'-end) and finally fused to the luciferase gene.

The expression plasmid FRB-ECFP-Sun5 was generated by amplifying Sun5/Spag $4 l$ from the construct HA-Spag4l-myc in pcDNA3.1(Ref: S.Hoyer-Fender). The primers pair used were Sun5-SacI-N-Rap and Sun5-KpnI-C-Rap (Tab.6.1.5). The PCR product was subcloned into pJET1.2 blunt vector (Fermentas). Afterward, Spag $4 l$ was cut-out with restriction enzymes SacI 
(at the 5'-end) and KpnI (at the $3{ }^{\prime}$-end). The plasmid $m E C F P$ - $F R B$-in pCRII (Ref: S.HoyerFender) was linearized with the restriction enzymes SacI and KpnI (both at the 3'-end). Ligation of Spag4l to $p C R I I-m E C F P-F R B$ resulted in the fusion of Spag4l to the C-terminal region of pCRII-mECFP-FRB. The coding sequence of FRB-ECFP-Spag $4 l$ was amplified with the primer pair ECFP-N-NheI and Sun5-KpnI-C-Rap and cloned pcDNA3.1 (-) C (Invitrogen) linearized with the restriction enzymes SacI and KpnI. All constructs were verified by sequencing.

\subsubsection{Sodium acetate/ethanol DNA precipitation}

Genomic DNA was concentrated and purified by adding 1/10 volumes of 3M sodium acetate and mixing thoroughly. Then, 2 volumes of $100 \%$ ethanol were added, mixed, and incubated overnight at $-20^{\circ} \mathrm{C}$. After incubation, the samples were centrifuged for 30 minutes at $4^{\circ} \mathrm{C}$ at top speed. The supernatant was discarded, and the DNA pellet was washed 2 times with $70 \%$ ethanol. The DNA pellet was air-dried and resuspended in $\mathrm{ddH}_{2} \mathrm{O}$.

\subsubsection{DNA purification}

The PCR product of interest was cut-out from the agarose gel and purified with NucleoSpin® Gel and PCR Clean-Up Kit following the manufacturer's instructions.

\subsubsection{Measurement of DNA and RNA concentrations}

DNA and RNA concentrations were obtained using either photo-spectrometer (Nanodrop, PeqLab) or Qubit ${ }^{\mathrm{TM}}$. Nanodrop estimated DNA concentration is based on UV light absorption at a wavelength of $260 \mathrm{~nm}$. A ratio of OD260/OD280 provided information about the purity of nucleic acids. Whereas Qubit ${ }^{\mathrm{TM}}$ estimate DNA or RNA concentration by measuring the fluorescence emitted by the binding to a specific dye. Qubit ${ }^{\mathrm{TM}}$ quantification reduces noise and gives more accurate results. DNA or RNA quantification was performed using specific Qubit ${ }^{\mathrm{TM}}$ fluorometers for dsDNA or RNA, as indicated in the instruction manual.

\subsubsection{Restriction enzyme digestion of DNA}

Specific restriction endonucleases were used to digest DNA. $1 \mu \mathrm{g}$ DNA was incubated with 1 unit of restriction enzyme in the appropriate reaction buffer, provided by the manufacturers, at recommended temperatures and time. Then, the DNA-enzyme digestion mix was inactivated at specific temperatures and time recommended for each enzyme. Finally, digested DNA fragments or vectors were analyzed by gel electrophoresis and purified using the NucleoSpin ${ }^{\circledR}$ Gel and PCR Clean-Up kit (2.2.2.4). 


\subsubsection{Vector DNA dephosphorylation}

After the restriction enzyme digestion was completed, phosphate groups were removed to prevent vector religation. DNA vector was treated with $1 \mu$ l Antarctic Phosphatase (AnP) (5U), $1 / 10$ vol of $10 \mathrm{X}$ Antarctic phosphatase reaction buffer, and ddH2O up to $50 \mu \mathrm{l}$. The dephosphorylation mix was incubated at $37^{\circ} \mathrm{C}$ for 30 minutes. The reaction was stopped by heat inactivation at $80^{\circ} \mathrm{C}$ for 2 minutes. Finally, the dephosphorylated vector was used to proceed with ligation.

\subsubsection{Ligation}

The ligation reaction was performed using T4-DNA ligase. The enzymatic activity of the DNA ligase catalyzes the formation of covalent phosphodiester linkages between the 3 ' hydroxyl end of one DNA fragment and the 5' phosphate end of another DNA fragment. Ligation reaction contained: $50 \mathrm{ng}$ vector DNA, X ng insert DNA, $2 \mu 1 \mathrm{~T} 4$ (10X) ligation buffer, $1 \mu 1 \mathrm{~T} 4 \mathrm{DNA}$ ligase $(5 \mathrm{U} / \mu \mathrm{l})$ in $20 \mu \mathrm{l}$ of total reaction volume. The ligation reaction was performed with a molar ratio of insert/vector of $3: 1$ or $1: 1$ followed by overnight incubation at $4{ }^{\circ} \mathrm{C}$.

\subsubsection{DNA Sequencing}

Plasmid or purified DNA was sent to Microsynth Seqlab (Göttingen) for sequencing.

\subsubsection{Total RNA isolation}

Total RNA was extracted either from NIH3T3 cells grown at a monolayer in cell culture dishes or mouse tissues. Mouse tissues include testis, liver, brain, ovary, kidney, epididymides, and spleen. A small piece of each tissue was triturated by using a mortar and continuously adding liquid nitrogen. The pulverized tissue was homogenized in $1 \mathrm{~mL}$ of peqGOLD RNAPure ${ }^{\mathrm{TM}}$ using a Dounce hand homogenizer (Tab. 2.8). The RNA extraction was essential, as described in the instruction manual. For RNA isolation from NIH3T3 cells, $1 \mathrm{~mL}$ of peqGOLD RNAPure ${ }^{\mathrm{TM}}$ was directly added to the cell monolayer and the extraction was carried out according to the instruction manual.

\subsubsection{RNA precipitation}

RNA precipitation was done by adding 0.1 volumes of $3 \mathrm{M}$ sodium acetate and 2 volumes of $100 \%$ ethanol. RNA was mixed thoroughly and incubated for 20 minutes at $-80^{\circ} \mathrm{C}$. After incubation, the samples were centrifuged for 20 minutes at $4{ }^{\circ} \mathrm{C}$, the supernatant was removed, and the RNA pellet was washed 2 times with $75 \%$ DEPC-treated ethanol (ice cold). The samples 
were centrifuged for 10 minutes at $4{ }^{\circ} \mathrm{C}$. The supernatant was removed, the pellet dried and resuspended in nuclease-free water.

\subsubsection{DNase treatment}

The DNase treatment was performed by adding $5 \mu 1$ 10X TURBO reaction buffer, $1 \mu \mathrm{l}$ (2 U) of TURBO DNase (MEGAscript ${ }^{\circledR}$ T7 kit (Tab. 2.8), $10 \mu \mathrm{g}$ of RNA and nuclease-free water up to $50 \mu$ l. The reaction mix was incubated at $37^{\circ} \mathrm{C}$ for 30 minutes. Thereafter, the DNase was inactivated by adding 0.1 volume ammonium acetate. Samples were stored at $-80^{\circ} \mathrm{C}$ for future cDNA synthesis.

\subsubsection{Phenol: Chloroform DNA/RNA precipitation}

DNA or RNA samples were treated with one volume of Ambion® Acid Phenol:Chloroform: IAA, premixed with Isoamyl alcohol (IAA) (25:24:1, pH 6.6/ 7.9). The mixture was vortexed for 20 seconds and centrifugated at room temperature for 5 minutes at $16,000 \mathrm{x} \mathrm{g}$. After centrifugation, the upper aqueous phase was transferred into a fresh $1.5 \mathrm{ml}$ tube. DNA or RNA was precipitated by the addition of 0.1 volume of $3 \mathrm{M}$ sodium acetate $(\mathrm{pH} 5.5)$ and $2.5 \mathrm{x}$ volumes of $100 \%$ ethanol. Thereafter, samples were incubated at $-20^{\circ} \mathrm{C}$ overnight. Then, samples were centrifuged at $16,000 \mathrm{x}$ g for 30 minutes at $4^{\circ} \mathrm{C}$. The supernatant was removed, and the pellet was washed twice with $70 \%$ ethanol. After the last wash, ethanol was removed entirely, and the pellet was dried at room temperature for 20-30 minutes. Finally, DNA or RNA pellet was resuspended in nuclease-free water. Samples were stored at $-20^{\circ} \mathrm{C}$ (DNA) or $-80^{\circ} \mathrm{C}$ (RNA) for future experiments.

\subsubsection{4 cDNA synthesis}

According to the manufacturer's instructions, the cDNA was synthesized using the Maxima First-Strand cDNA Synthesis Kit (Tab. 2.8). cDNA was used as the template either for RT-PCR or qRT-PCR.

\subsubsection{RT-PCR}

RT-PCR was performed according to each primer pair parameters and the size of the expected fragments (Tab. 6.1.2). PCR amplification was carried out using either Mango Taq DNA polymerase, Taq polymerase, or Phusion polymerase. 


\subsubsection{6 qRT-PCR}

qRT-PCR was performed either with All-in-One ${ }^{\mathrm{TM}}$ qPCR mix or HOT FIREpol ${ }^{\circledR}$ EvaGreen ${ }^{\circledR}$ qPCR using the CFX96 ${ }^{\mathrm{TM}}$ Real-Time PCR System (Bio-Rad Laboratories, Hercules, CA, USA). cDNA from a wild type mouse was used to calculate primer efficiency. Relative fold gene quantity was calculated by the delta-delta $\mathrm{Ct}$ method $\left(2^{-\Delta \Delta C t}\right)$ using $m H P R T$ as a reference gene. Primer sequences are given in table 6.1.3.

\subsubsection{PCR}

DNA was amplified using MangoTaq ${ }^{\mathrm{TM}}$ DNA, Phusion, or Advantage 2 Polymerase. Different reactions were performed following the manufacturer's instructions. PCR products were evaluated by agarose gel electrophoresis.

\subsubsection{Agarose gel electrophoresis of DNA}

PCR products were evaluated by gel electrophoresis using either $1 \%$ or $2 \%$ agarose dissolved in 1X-TAE buffer and Serva DNA Stain G. Gel was solidified and inserted into a chamber covered with 1X-TAE buffer. DNA samples were mixed with loading dye, loaded onto the gel, and separated at $100 \mathrm{~V}-120 \mathrm{~V}$.

\subsubsection{Biochemical methods}

\subsubsection{Protein extraction from mammalian cells}

Mammalian cells were washed with 1XPBS, trypsinized, and centrifuged for $3 \mathrm{~min}$ at $2000 \mathrm{x} \mathrm{g}$. The cell pellet was washed with 1X-PBS and cellular concentration was measured using a Neubauer haemocytometry. $1 \times 10^{6}$ cells were resuspended in $32 \mu 1$ 1x-PBS. Protein denaturation was performed by adding $4 \mu \mathrm{l}$ of $8 \mathrm{X}$ denaturation buffer and heating at $95^{\circ} \mathrm{C}$ for 10 minutes.

\subsubsection{SDS-Polyacrylamide gel electrophoresis}

Polyacrylamide gel electrophoresis (PAGE) (Laemmli, 1970) was used to separate proteins based on their size. For that purpose, proteins were denatured to avoid protein folding and give them a negative charge. SDS gels were prepared using a stock solution of $40 \%$ acrylamide/ bisacrylamide in a ratio of 29:1. The acrylamide concentration chosen for resolving gel preparation $(10 \%, 12 \%$, or $15 \%)$ was adapted to the expected molecular size range, whereas the stacking gel was prepared at a concentration of $10 \%$. 
Protein samples were loaded onto the SDS-PAGE and separated at $80 \mathrm{~V}$ for $30 \mathrm{~min}$. When the loading dye has entered the resolving gel, the voltage turned to $100 \mathrm{~V}$ for another 1.5 hours.

\begin{tabular}{|c|c|c|c|c|c|}
\hline \multicolumn{2}{|c|}{ Stacking gel } & \multicolumn{4}{|c|}{ Separating gel } \\
\hline & $10 \mathrm{ml}$ & & $10 \%$ & $12 \%$ & $15 \%$ \\
\hline 40\% AA Bis Mix & $1.25 \mathrm{ml}$ & 40\% AA Bis Mix & $5 \mathrm{ml}$ & $6 \mathrm{ml}$ & $7.5 \mathrm{ml}$ \\
\hline $\mathrm{ddH}_{2} \mathrm{O}$ & $6.3 \mathrm{ml}$ & $\mathrm{ddH}_{2} \mathrm{O}$ & $9.6 \mathrm{ml}$ & $8.6 \mathrm{ml}$ & $7.1 \mathrm{ml}$ \\
\hline 1M Tris- $\mathrm{HCl} \mathrm{pH} 6.8$ & $1.25 \mathrm{ml}$ & $1.5 \mathrm{M}$ Tris- $\mathrm{HCl} \mathrm{pH} 8.8$ & $5 \mathrm{ml}$ & $5 \mathrm{ml}$ & $5 \mathrm{ml}$ \\
\hline $10 \%$ SDS & $100 \mu \mathrm{l}$ & $10 \%$ SDS & $200 \mu 1$ & $200 \mu \mathrm{l}$ & $200 \mu 1$ \\
\hline $10 \%$ APS & $100 \mu \mathrm{l}$ & $10 \%$ APS & $200 \mu \mathrm{l}$ & $200 \mu 1$ & $200 \mu 1$ \\
\hline TEMED & $100 \mu \mathrm{l}$ & TEMED & $20 \mu \mathrm{l}$ & $20 \mu \mathrm{l}$ & $20 \mu 1$ \\
\hline
\end{tabular}

Table 2.10. Volumes needed for the preparation of two stacking and separating gels

\subsubsection{Western-blot (Immunoblot)}

The Western-blot is defined as the transfer of proteins onto a nitrocellulose membrane (Towbin et al., 1979), followed by protein detection using specific antibodies. The nitrocellulose membrane (Amersham Hybond-ECL, GE Healthcare) was placed together with the SDS-gel between two Whatman filter papers. This set was placed between two sponges into a cassette. Finally, the cassette was placed into the blot chamber (Bio-Rad) containing Towbin buffer. Protein transfer was performed by applying $100 \mathrm{~V}$ for 1 hour.

\subsubsection{Immunological protein detection}

Membranes were used for antibody detection. Membranes were incubated for one hour at room temperature in blocking solution (5\% dry milk in TBST). Afterward, membranes were incubated with primary antibodies diluted in blocking solution at $4{ }^{\circ} \mathrm{C}$ overnight. After incubation with the first antibody, membranes were washed with TBST buffer for 45 minutes and replacing the TBST buffer every 5 minutes. Then, membranes were incubated with horseradish peroxidase-conjugated secondary antibodies diluted in blocking solution for 45 minutes at room temperature. Unbound antibodies were removed by washing the membranes for 30 minutes with TBST buffer, replacing the buffer every 5 minutes. The HRP conjugated secondary antibodies were detected by chemiluminescence (ECL). Light emission from proteins was detected by ChemiDoc ${ }^{\mathrm{TM}}$ Imaging System. The optimal exposure time of each membrane was always adapted to the experimental needs. 


\subsubsection{Nitrocellulose membrane stripping}

The nitrocellulose membrane was reevaluated. For this purpose, the primary and secondary antibodies were first removed. Nitrocellulose membranes were stripped accordingly: 1. blots were incubated in $1 \mathrm{X}$-PBS for 10 minutes at room temperature with constant agitation, 2. the PBS buffer was exchanged for stripping solution (see 2.1.9) followed by incubation for 8 minutes, 3. the stripping solution was discarded, and membranes were washed twice with 1XPBS for 10 minutes each. Thereafter, membranes were washed twice with TBST buffer for 5 minutes each, and unspecific binding sites were blocked with 5\% dry milk powder in TBST. The nitrocellulose membrane is now ready for the antibody incubation.

\subsubsection{Co-IP (Co-immunoprecipitation)}

Cells were reseeded and transfected, as described above. 18-24 hours post-transfection, cells were harvested by trypsinization, collected and washed twice with ice-cold 1X-PBS. Cellular pellet was resuspended in $1 \mathrm{ml}$ lysis buffer for CoIP, vortexed, and incubated on ice for 20 minutes. After incubation, cell lysate was passed 10 times through a 21.5-gauge needle followed by 3 times sonication for 45 seconds each. Samples were centrifuged at $15,000 \times \mathrm{g}$ for 15 minutes and the supernatant was used for CoIP analysis. $50 \mu \mathrm{l}$ of supernatant was stored as input, whereas the remnant protein lysate was split into equal parts to perform immunoprecipitation with specific and control antibodies. Incubation with fishing antibodies was carried out on a rolling platform for 1 hour at room temperature. Then, protein $\mathrm{G}$ beads were washed with lysis buffer for CoIP and subsequently added to the protein lysate-antibody mixture and incubated overnight at $4^{\circ} \mathrm{C}$ on a rolling platform. After overnight incubation, beads containing proteins and antibodies were washed 4 times with $1 \mathrm{ml}$ lysis buffer. Finally, beads were resuspended in $50 \mu \mathrm{l} 1 \mathrm{X}$ denaturation buffer, whereas the supernatant of $50 \mu \mathrm{l}$ was resuspended in $50 \mu 12 \mathrm{X}$ denaturation buffer for SDS-PAGE electrophoresis and boiled at $95^{\circ} \mathrm{C}$ for 10 minutes.

\subsubsection{Crosslinking reaction and harvesting adherent cells}

Twenty-four hours post-transfection, cell culture dishes were placed on ice, and the medium was removed. Adherent cells were washed two times with 1X-PBS. Then, fresh 1X-PBS was added to the cells, followed by incubation at $4{ }^{\circ} \mathrm{C}$ with concurrent exposition with or without

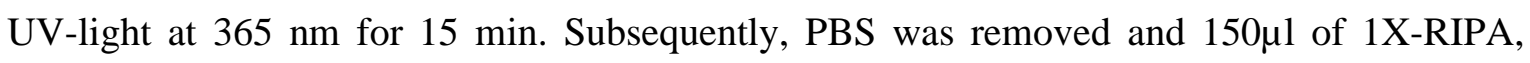
containing protease inhibitors, was added (Tab. 2.6), followed by incubation on ice for 10 minutes under slow horizontal movements. Thereafter, cells were scratched using a cell scraper. The cell lysate was transferred into a $1.5 \mathrm{ml}$ tube and centrifuged at $11.000 \mathrm{rpm}$ for $10 \mathrm{~min}$ at 
$4^{\circ} \mathrm{C}$. After centrifugation, the supernatant was transferred into a new $1.5 \mathrm{ml}$ tube and $25 \mu \mathrm{l}$ of 5X LPP buffer was added to $100 \mu 1$ of the supernatant. Samples were heated for $15 \mathrm{~min}$ at $95^{\circ} \mathrm{C}$ and stored at $-20^{\circ} \mathrm{C}$ for Western-blot analysis.

\begin{tabular}{|l|l|}
\hline pLMNC-T488-3X-FLAG & Amber stop codon TAG 488 \\
\hline$p L M N C-T 534-3 X-F L A G$ & Amber stop codon TAG 534 \\
\hline$p L M N C-R 470-3 X-F L A G$ & Amber stop codon TAG 470 \\
\hline$p 4 X t R N A_{p \text { Bpa }}$ & \\
\hline
\end{tabular}

Table 2.11. Plasmids and tRNA for UAA incorporation

\subsubsection{Cell biological methods}

\subsubsection{Propagation and subculture of eukaryotic cells}

NIH3T3 and HEK293 cells were cultivated in Dulbecco's Eagle Medium (DMEM) enriched with $10 \%$ fetal bovine serum and 1\% penicillin/streptomycin (complete DMEM). Cells were maintained at $37^{\circ} \mathrm{C}$ and $5 \% \mathrm{CO}_{2}$. Cells were propagated by removing the old medium and washing them with sterile 1 X-PBS. Cells were incubated with $0.25 \%$ trypsin, followed by incubation for 3 minutes at $37^{\circ} \mathrm{C}$. Trypsin was inactivated by adding complete DMEM and a large part of the solution aspirated. The remaining cells were propagated further by adding fresh complete DMEM.

\subsubsection{Defrosting and freezing of eukaryotic cells}

For long-term storage, harvested cells were resuspended in freezing medium containing $20 \%$ DMSO. Cells were collected in cryo-vials and first incubated for 2 hours at $-20^{\circ} \mathrm{C}$ followed by transfer to $-80^{\circ} \mathrm{C}$ overnight. Finally, cryo-vials were stored in liquid nitrogen. For revitalization, frozen cells in cryo-vials were prewarmed in a water bath for 3 minutes at $37^{\circ} \mathrm{C}$ and subsequently diluted with prewarmed medium.

\subsubsection{Transfection}

NIH3T3 and HEK293 cells were transfected with EndoFectin ${ }^{\text {TM }}$ Max transfection reagent following the manufacturer's instructions. Thereafter, cells were incubated at $37^{\circ} \mathrm{C}$ and $5 \% \mathrm{CO}_{2}$ for 18-24 hours. 


\subsubsection{Immunocytology on sperm suspension}

Fresh testes isolated from mice (C57BL/6) were washed with 1X-PBS, the tunica albuginea was removed, and germ cells were suspended by mincing and pipetting in 3.7\% PFA. Cell suspensions were spread on Superfrost Plus (Menzel Glas, Braunschweig) followed by incubation in a humid chamber for 20 minutes. Cells were permeabilized with $0.3 \%$ Triton X100 (in 1X-PBS) for 10 minutes at room temperature. After washing the samples with 1X-PBS, they were incubated in PBT blocking solution for 1 hour. Proteins of interest were stained with appropriate primary antibodies overnight at $4^{\circ} \mathrm{C}$. Excess of primary antibodies was removed by washing the samples 3 times with $1 \mathrm{X}$-PBS. Thereafter, samples were incubated with appropriate secondary antibodies for 45 minutes at $37^{\circ} \mathrm{C}$. Finally, samples were washed three times in $1 \mathrm{X}$ PBS for 5 minutes each, the mounting medium (Fluoromount- $\mathrm{G}^{\mathrm{TM}}$ ) was added, and slides were covered with a coverslip and sealed with nail polish. Samples were analyzed by the fluorescence microscope LSM 750 or 980 (ZEISS) and processed using Adobe Photoshop 7.0.

\subsubsection{Immunocytology}

Transfected or untransfected NIH3T3 cells were cultivated on coverslips in six-well plates containing an initial concentration of $2.5 \times 10^{5}$ or $3 \times 10^{5}$ cells per well. After 24 hours, cells were washed 3 times with 1X-PBS and fixed in $3.7 \%$ PFA in 1X-PBS for 20 minutes at $4^{\circ} \mathrm{C}$. Alternatively, cells were fixed with $100 \%$ methanol for 10 minutes at $-20^{\circ} \mathrm{C}$. After 3 times washing with $1 \mathrm{X}-\mathrm{PBS}$, specimens were permeabilized in $0.3 \%$ Triton X-100 (in 1X-PBS) for 10 minutes at room temperature. Samples were washed 3 times with 1X-PBS. Another fixative option was to fix cells with $3 \%$ Glyoxal for 30 minutes at $4^{\circ} \mathrm{C}$, followed by incubation with $\mathrm{NH}_{4} \mathrm{Cl}$ for 30 minutes at $4^{\circ} \mathrm{C}$. Permeabilization was performed using $0.2 \%$ Triton X-100 (in $1 \mathrm{X}$ PBS) for 10 minutes at room temperature. After permeabilization, unspecific antibody bindings were blocked with PBT for 1 hour at room temperature. Subsequently, primary antibodies were added to the samples and incubated for 1 hour at $37^{\circ} \mathrm{C}$. Thereafter, cells were washed 3 times with $1 \mathrm{X}$-PBS for 5 minutes each. Afterward, secondary antibodies were added, followed by incubation for 45 minutes at $37^{\circ} \mathrm{C}$. DNA was counterstained with DAPI. Cells were washed 3 times with 1X-PBS and covered with mounting medium (Fluoromount-G ${ }^{\mathrm{TM}}$ ). To protect samples from drying, coverslip borders were sealed with nail polish. Slides were stored at $4{ }^{\circ} \mathrm{C}$ until they were analyzed by laser scanning microscopy (Zeiss LSM 750 or LSM 980) and processed using Adobe Photoshop 7.0. 


\subsubsection{Generation of Spag4l-deficient mice}

\subsubsection{Production of the targeting vector}

Homologous recombined mouse embryonic stem cells (Sun5tm1(KOMP)Vlcg, KOMP/Velocigene project: VG16078) in which the insertion of a replacement vector deleted the Spag4l2/Sun5 gene were purchase from Velocigene Regeneron Pharmaceuticals.Inc (http://velocigene.com/komp/detail/16078). The Sun5 gene comprises in mouse 12 exons which were entirely disrupted in the embryonic stem cell line and replaced by the reporter gene LacZ and the resistant gene Neomycin. The cassette deleted the region 153856177 to 153870995 , which localizes to chromosome 2. The presence of the targeting vector in ES cells was tested by PCR and qPCR.

\subsubsection{Generation of Sun5 knockout mice}

Recombinant ES cells were propagated by Prof. Dr. Ibrahim Adham (Institute of Human Genetics, University Medical Center Göttingen). Chimeric mice were generated by microinjection of recombinant ES cells into embryos of the C57B16 mouse strain by the animal facility of the Max Planck Institute of Experimental Medicine, Göttingen. Heterozygous Sun5deficient mice were generated by breeding of chimeras carrying the replacement cassette and C57B16 mice. Chimeras and heterozygous Sun5-deficient mice were genotyped by PCR and qPCR.

\subsubsection{Genomic DNA extraction}

Genomic DNA was prepared using either KAPA ${ }^{\mathrm{TM}}$ Mouse Genotyping Kit, NID buffer, or Viagen-DirectPCR ${ }^{\circledR}$-Tail. ES cell pellet, mice tail-tips, or ear punches were incubated in lysis solution containing $1 \mu \mathrm{l}$ of KAPA express enzyme, 10X KAPA buffer, and PCR-grade water. Lysis was performed by incubation in a heating block for 1 hour at $75^{\circ} \mathrm{C}$ followed by enzyme inactivation for 5 minutes at $95^{\circ} \mathrm{C}$.

To isolate genomic DNA from mice tail-tip biopsies, tails were placed into a $1.5 \mathrm{ml}$ tube containing $300 \mu \mathrm{l}$ Viagen-DirectPCR ${ }^{\circledR}$-Tail buffer (Tab. 2.8) and $0.4 \mathrm{mg} / \mathrm{ml}$ of proteinase $\mathrm{K}$. Samples were incubated overnight at $55^{\circ} \mathrm{C}$ under continuous agitation. Afterward, proteinase $\mathrm{K}$ was inactivated by incubation at $85^{\circ} \mathrm{C}$ for 45 minutes.

Alternatively, mice tail-tips or ear punches were incubated $300 \mu$ l NID buffer containing proteinase $\mathrm{K}$ (Tab. 2.6) followed by incubation at $56^{\circ} \mathrm{C}$ overnight. Then, proteinase $\mathrm{K}$ was inactivated by incubation at $95^{\circ} \mathrm{C}$ for 15 minutes. 
Finally, DNA samples were quickly centrifugated, the supernatant transferred into a new $1.5 \mathrm{ml}$ tube, and DNA was precipitated using sodium acetate/ethanol (2.2.2.3).

\subsubsection{Genotyping of mice using PCR}

gDNA from ES cells or biopsies from chimeras and their progenies were used to investigate the replacement of the gene Sun5 by the integration cassette. Thus, gDNA was used as a PCR template using different primer pairs combinations (Tab. 6.1.7).

ES cells were genotyped by PCR using two primer pairs. SunUp/LacInRev was used to amplify a fragment of $562 \mathrm{bp}$ comprising the upstream region of the wild-type allele and $132 \mathrm{bp}$ of the recombinant allele. NeoFwd/SunDown was used to amplify $101 \mathrm{bp}$ of the recombinant allele and $406 \mathrm{bp}$ of the 3 ' region of the wild-type allele.

Several primer sets were used to detect the transmission of the Sun5 replacement allele in chimeras and heterozygous mice. SunUp/LacInRev is located at the $5^{\prime}$ region as described. LacInF/LacInR are located in the reporter gene LacZ. Different set of primers were used to detect the selection marker neomycin, inter alia NeoInF/NeoInR, NeomycinFor3/NeoInR, NeomycinFor/NeoSplitRev and NeomycinFor/NeoInR. Alike, NeoFwd/SunDown, Geno2For/Geno2Rev, and NeomycinFor3/Genotype2Rev locate downstream of the targeting vector or in its 3 ' region (Fig. 3.24).

PCR reactions were performed using Mango Taq DNA polymerase and primer specific conditions as described in table 2.12 .

\begin{tabular}{cccc}
\hline Step & Temperature & Time & Repetitions \\
\hline Initial denaturation & $95^{\circ} \mathrm{C}$ & $3 \mathrm{~min}$ & \\
\hline Denaturation & $95^{\circ} \mathrm{C}$ & $30 \mathrm{sec}$ & \multirow{2}{*}{45 cycles } \\
Annealing & $\begin{array}{c}\text { Primer TM } \\
(\text { Appendix } 6.1)\end{array}$ & $30 \mathrm{sec}$ & \\
\hline Extension & $72^{\circ} \mathrm{C}$ & specific to each \\
& & fragment length \\
\hline Final extension & $72^{\circ} \mathrm{C}$ & 10 min \\
\hline
\end{tabular}

Table 2.12. PCR conditions used for genotyping 


\subsubsection{Genotyping of mice using qPCR}

ES cells and mice tail-tips were genotyped by quantitative Real-Time PCR using CFX96 ${ }^{\mathrm{TM}}$ Real-Time PCR System (Bio-Rad Laboratories, Hercules, CA, USA). gDNA was isolated as described (2.2.5.3) and used as the template for qPCR using different primer pair combinations. Primer sets (Tab. 6.1.8) were designed for the amplification of either exon 7 or 9. Primer efficiency was evaluated by the generation of a standard curve with wild-type mouse gDNA. The standard curve was obtained by using serial dilutions of the template.

To perform the qPCR, gDNA from a wild-type mouse was used as internal control, whereas Prml and $O d f 1$ were used as reference genes to calculate the fold change $\left(\log 2^{-\Delta \Delta C t}\right)$.

Internal control, samples, and non-template controls were prepared in triplicate and mixed with the following components: 2X KAPA SYBR® FAST qPCR master mix or HOT FIREpol® EvaGreen ${ }^{\circledR}$ qPCR Mix Plus (ROX), $10 \mu \mathrm{M}$ forward and reverse primers each, gDNA template and $\mathrm{ddH} 2 \mathrm{O}$ water up to $10 \mu \mathrm{l}$ total reaction volume. qPCR reactions were performed using the conditions described in table 2.13 .

\begin{tabular}{cccc}
\hline Step & Temperature & Time & Repetitions \\
\hline Initial denaturation & $95^{\circ} \mathrm{C}$ & $3 \mathrm{~min}$ & \\
\hline Denaturation & $95^{\circ} \mathrm{C}$ & $3 \mathrm{sec}$ & \multirow{2}{*}{40 cycles } \\
\hline Annealing & $57^{\circ} \mathrm{C}$ & $20 \mathrm{sec}$ & \\
\hline Extension & $72^{\circ} \mathrm{C}$ & $15 \mathrm{sec}$ & \\
\hline
\end{tabular}

Table 2.13. qPCR conditions used for genotyping 


\section{Results}

\subsection{Mutation screening in patients with acephalic spermatozoon phenotype}

Teratozoospermia (TZI) describes sperm malformations that cause male infertility, including abnormalities of the head and middle/principal piece. The syndrome acephalic or pinhead spermatozoa is characterized by a fragile connecting piece disrupting the connection between sperm head and tail. Albeit these disorders have genetic origins, the molecular components have been scarcely described (Perotti et al., 1981; WHO, 2010; Chemes et al., 1999; Chemes, 2000).

Genetic studies in patients and the generation of knock-out mouse models combined with the ultrastructural evaluations of spermatozoa have enabled the identification of essential genes required for the tight junction between sperm head and tail. The disruption of HTCA or manchette has been ascribed to alterations of Prss21, Oaz3, Cntrob, Ift88, Spata6, Odf1, Hook1, Arl3, Stk36, Rim-bp3, and Sun5 (Mendoza-Lujambio, 2002; Netzel-Arnett et al., 2009; Tokuhiro et al., 2009; Zhou et al., 2009; Liška et al., 2013; Qi et al., 2013; Nozawa et al., 2014; Yang et al. 2012; 2014; Yuan et al., 2015; Zhu et al., 2016).

Most of these genes have been identified in mouse models. This is the case of Odfl that encodes the ODF1 protein. ODF1 is an essential component of the HTCA, and its disruption in mice induces the detachment of the sperm head to the tail. However, no mutations have been reported in infertile patients suffering from the acephalic spermatozoan syndrome. Thus, it raises the question if Odf1 mutations might be causative for male infertility in humans. Therefore, I analyzed the DNA of two infertile patients who had been diagnosed with acephalic spermatozoan phenotype. DNA samples were kindly provided by Prof. Dr. med. Héctor Chemes (Center for Research in Endocrinology, Buenos Aires Children's Hospital, Argentina), who also got the informed consent of the patients. The screening for mutations was carried out on the candidate genes Odf1, Hook1, and Sun5. These genes have been previously reported to play a key role in the sperm head-tail junction. 


\subsubsection{Patients with decapitated spermatozoa carried normal sequence variations of the gene Odf1 compared to the reference wild-type sequence}

ODF1 is a component of the sperm tail outer dense fibers (ODFs) situated parallel to the axoneme. They contribute to the tensile forces of the sperm by providing elasticity to the sperm tail during the flagellar beat. The analyses of spermatozoa from Odfl-deficient mice have revealed reduced sperm motility and sperm decapitation. Furthermore, the depletion of Odf1 caused abnormal mitochondrial arrangement and ODF organization and affected the proper attachment of the sperm head and tail (Fawcett, 1975; Baltz et al., 1990a; Yang et al., 2012; 2014).

Odf1 gene consists of two exons. Exon 1 comprises 492 bp, whereas exon 2 consists of 596 bp (Hofferbert et al., 1993). Thus, the DNA from two unrelated anonymous patients with acephalic spermatozoan syndrome, named P17 and P19, were used to amplify both exons of the human Odf1 gene. Exons were amplified using the pair of primers Odf1-Homo-Exon1-For/ Odf1Homo-Exon1-Rev and Odf1-Homo-Exon2-Forward/ Odf1-Homo-Exon2-Reverse (Tab. 6.1.1). The PCR product of exon 1 exhibited a size of $590 \mathrm{bp}$, and amplification of exon 2 resulted in two different fragments of $590 \mathrm{bp}$ and $635 \mathrm{bp}$, respectively. All PCR products were cloned into the vector pJET1.2/blunt (Fermentas), and the DNA isolated from 5 up to 9 individual colonies were sequenced. The obtained sequences were aligned to the human reference NG_028006.1 by using Multiple Sequence Alignment Clustal Omega. Sequences from exon 1 exhibited a normal coding region in both patients, being identical to the reference sequence (Fig.3.1 - 3.2). Regarding exon 2, two slightly different amino acid sequences were detected in each patient, which corresponded to the variations described as CSP and CNP (Fig. 3.3 - 3.4) (Hofferbert et al., 1993). In humans, in its C-terminal region ODF1 possesses conserved cysteine residues in a variable repetitive tripeptide motif of C-X-P. The tripeptide motif variation is either CSP or $\mathrm{CNP}$ resulting from two nucleotide exchanges (AGC $\rightarrow$ AAC) and (CCC $\rightarrow \mathrm{CCG}$ ). Thus, humans are either homozygous or heterozygous for the two OdfI alleles. Allele I is shorter and less frequent than allele II. Besides that, allele II is encoded by three additional motifs at the $\mathrm{COOH}$-terminal end. However, these variations do not cause a pathologic sperm phenotype (Burfeind \& Hoyer-Fender, 1991; Hofferbert et al., 1993; Hoyer-Fender et al., 1995). To conclude, neither patient P17 nor patient P19 carry harmful mutations in the OdfI gene. Therefore, in these cases, $O d f 1$ is not responsible for the acephalic spermatozoa syndrome. 


\begin{tabular}{|c|c|c|c|}
\hline Odf1 & \multicolumn{2}{|c|}{ MAALSCLLDSVRRDIKKVDRELRQLRCIDEFSTRC LCDLYMHPYCCCDLHPY PYCLCYSK } & 60 \\
\hline P17-C1on 4 & \multicolumn{2}{|c|}{ MAALSC LLDSVRRDIKKVDRELRQLRCIDEFSTRCLCDLYMHPYCCCDLHPY PYCLCYSK } & \\
\hline P17-C1on 5 & \multirow{2}{*}{\multicolumn{2}{|c|}{$\begin{array}{l}\text { MAALSC LLDSVRRDIKKVDRELRQLRCIDEFSTRC LCDLYMHPYCCCDLHPY PYCLCYSK } \\
\text { MAALSCLLDSVRRDIKKVDRELRQLRCIDEFSTRCLCDLYMHPYCCCDLHPY PYCLCYSK }\end{array}$}} & 60 \\
\hline P17-Clon 6 & & & \\
\hline P17-C1on16 & \multirow{2}{*}{\multicolumn{2}{|c|}{$\begin{array}{l}\text { MAALSC LLDSVRRDIKKVDRELRQLRCIDEFSTRC LCDLYMHPYCCCDLHPY PYCLCYSK } \\
\text { MAALSCLLDSVRRDIKKVDRELRQLRCIDEFSTRCLCDLYMHPYCCCDLHPY PYCLCYSK }\end{array}$}} & 60 \\
\hline P17-Clon21 & & & 60 \\
\hline P17-C1on23 & \multicolumn{2}{|c|}{ MAALSCLLDSVRRDIKKVDRELRQLRCIDEFSTRCLCDLYMHPYCCCDLHPY PYCLCYSK } & 60 \\
\hline P17-C1on24 & \multicolumn{2}{|c|}{ MAALSC LLDSVRRDIKKVDRELRQLRCIDEFSTRCLCDLYMHPYCCCDLHPY PYCLCYSK } & 60 \\
\hline P17-C1on29 & \multicolumn{2}{|c|}{ MAALSCLLDSVRRDIKKVDRELRQLRCIDEFSTRC LCDLYMHPYCCCDLHPY PYCLCYSK } & 0 \\
\hline P17-Clon 30 & \multicolumn{3}{|c|}{ 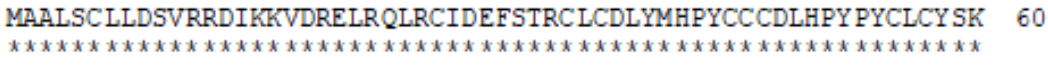 } \\
\hline Odf1 1 & RSRSCGLCDLY PCCLCD YKLYCLRPSLRS LERKAI RAIEDE KRELAK & 107 & \\
\hline P17-C1on 4 & RSRSCGLCDLYPCCLCDYKLYCLRPSLRS LERKAI RAIEDE KRELAK & 107 & \\
\hline P17-C1on5 & RSRSCGLCDLY PCCLCD YKLYCLRPSLRS LERKAI RAIEDE KRELAK & 107 & \\
\hline P17-C1on6 & RSRSCGLCDLY PCCLCDYKLYCLRPSLRS LERKAI RAIEDE KRELAK & 107 & \\
\hline P17-Cion16 & RSRSCGLCDLY PCCLCD YKLYCLRPSLRS LERKAI RAIEDE KRELAK & 107 & \\
\hline P17-C1on21 & RSRSCGLCDLYPCCLCDYKLYCLRPSLRS LERKAI RAIEDE KRELAK & 107 & \\
\hline P17-C1on23 & RSRSCGLCDLY PCCLCDYKLYCLRPSLRS LERKAI RAIEDE KRELAK & 107 & \\
\hline P17-Clon24 & RSRSCGLCDLY PCCLCDYKLYCLRPSLRS LERKAI RAIEDE KRELAK & 107 & \\
\hline P17-Clon 29 & RSRSCGLCDLY PCCLCDYKLYCLRPSLRS LERKAI RAIEDE KRELAK & 107 & \\
\hline P17-C1on30 & 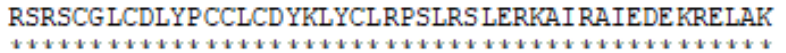 & 107 & \\
\hline
\end{tabular}

Figure 3.1. Amino acid sequence alignment of ODF1-exon 1 from patient $\mathbf{n}^{\circ} 17$. The nine clones analyzed showed all an amino acid sequence identical to the reference ODF1 (ref. sec NC_000008.11).

\begin{tabular}{|c|c|c|}
\hline Odf1 1 & MAALSC LLDSVRRDIKKVDRELRQLRCIDEFSTRC LCDLYMHPYCCCDLHPY PYCLCYSK & 60 \\
\hline P19-Clonl & MAALSCLLDSVRRDIKKVDRELRQLRCIDEFSTRCLCDLYMHPYCCCDLHPY PYCLCYSK & 60 \\
\hline P19-Clon 3 & MAALSCLLDSVRRDIKKVDRELRQLRCIDEFSTRCLCDLYMHPYCCCDLHPY PYCLCYSK & 60 \\
\hline P19-Clon7 & MAALSCLLDSVRRDIKKVDRELRQLRCIDEFSTRCLCDLYMHPYCCCDLHPY PYCLCYSK & 60 \\
\hline P19-Clon 11 & MAALSCLLDSVRRDIKKVDRELRQLRCIDEFSTRCLCDLYMHPYCCCDLHPY PYCLCYSK & 60 \\
\hline P19-Clon 12 & MAALSCLLDSVRRDIKKVDRELRQLRCIDEFSTRCLCDLYMHPYCCCDLHPY PYCLCYSK & 60 \\
\hline P19-Clon19 & MAALSCLLDSVRRDIKKVDRELRQLRCIDEFSTRCLCDLYMHPYCCCDLHPY PYCLCYSK & 60 \\
\hline P19-Clon 21 & MAALSCLLDSVRRDIKKVDRELRQLRCIDEFSTRCLCDLYMHPYCCCDLHPY PYCLCYSK & 60 \\
\hline P19-Clon26 & MAALSC LLDSVRRDIKKVDRELRQLRCIDEFSTRC LCDLYMHPYCCCDLHPY PYCLCYSK & 60 \\
\hline P19-C1on 36 & 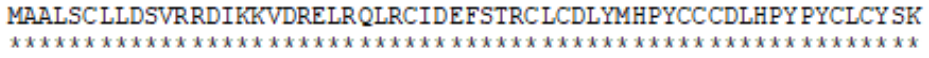 & 60 \\
\hline Odf1 & RSRSCGLCDLY PCCLCDYKLYCLRPSLRS LERKAI RAIEDEKRELAK 107 & \\
\hline P19-Clonl & RSRSCGLCDLYPCCLCDYKLYCLRPSLRS LERKAI RAIEDE KRELAK 107 & \\
\hline P19-Clon3 & RSRSCGLCDLYPCCLCD YKLYCLRPSLRS LERKAI RAIEDE KRELAK 107 & \\
\hline P19-Clon 7 & RSRSCGLCDLYPCCLCD YKLYCLRPSLRS LERKAI RAIEDEKRELAK 107 & \\
\hline P19-Clon11 & RSRSCGLCDLY PCCLCD YKLYCLRPSLRS LERKAI RAIEDEKRELAK 107 & \\
\hline P19-C1 on 12 & RSRSCGLCDLYPCCLCD YKLYCLRPSLRS LERKAI RAIEDE KRELAK 107 & \\
\hline P19-Clon 19 & RSRSCGLCDLYPCCLCDYKLYCLRPSLRS LERKAI RAIEDE KRELAK 107 & \\
\hline P19-C1 on 21 & RSRSCGLCDLYPCCLCDYKLYCLRPSLRS LERKAI RAIEDEKRELAK 107 & \\
\hline P19-C1on26 & RSRSCGLCDLY PCCLCD YKLYCLRPSLRS LERKAI RAIEDEKRELAK 107 & \\
\hline P19-Clon 36 & RSRSCGLCDLYPCCLCD YKLYCLRPSLRS LERKAI RAIEDE KRELAK 107 & \\
\hline & 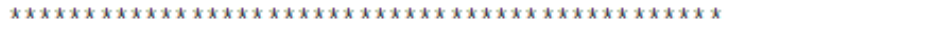 & \\
\hline
\end{tabular}

Figure 3.2. Amino acid sequence alignment of ODF1-exon1 from patient $\mathbf{n}^{\circ} \mathbf{1 9}$. The nine clones analyzed showed all an amino acid sequence identical to the reference ODF1 (ref. sec NC_000008.11). 


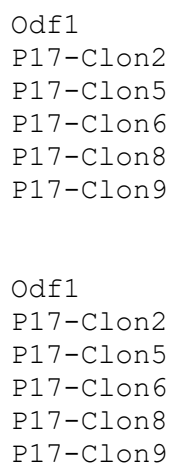

Figure 3.3. Amino acid sequence alignment of ODF1-exon 2 from patient $\mathbf{n}^{\circ} 17$. Two out of five clones showed the variant motif CSP (clones 2 and 5; yellow) that corresponded to the less frequent allele I. Clones 6,8 , and 9 (green) have the variant motif CNP and contain three additional CNP motifs, which corresponds to the allele II (ref. sec NG_028006.1).

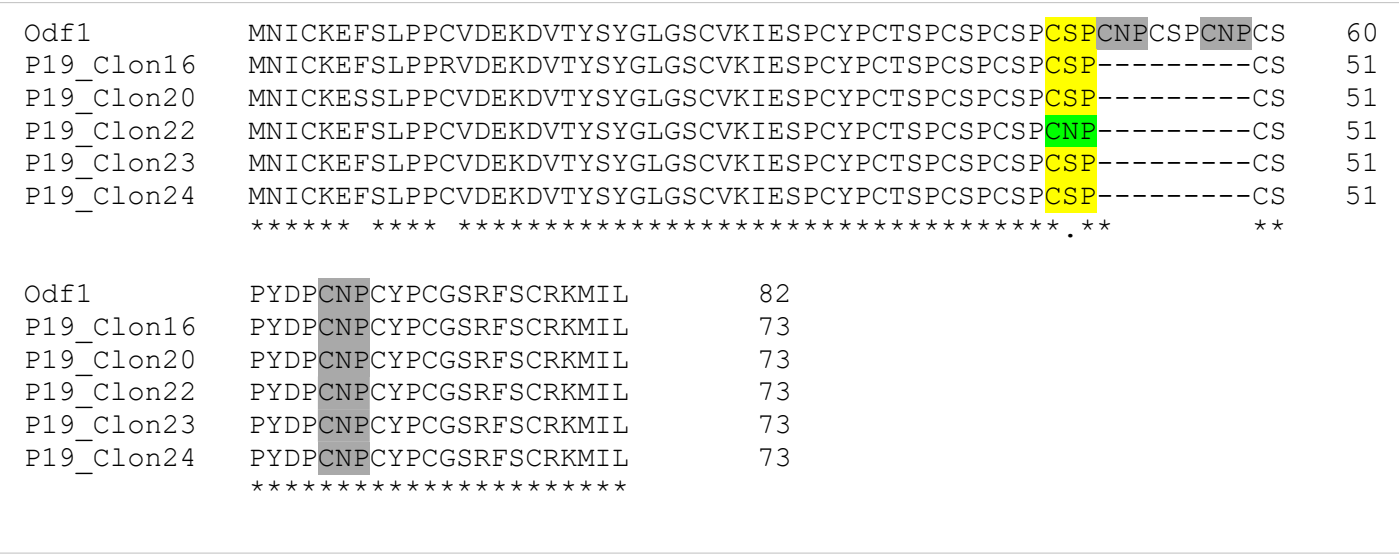

Figure 3.4. Amino acid sequence alignment of ODF1-exon 2 from patient $n^{\circ} 19$. Four out of five clones carried the variant motif CSP (clones 16, 20, 23, and 24; yellow) whereas clone 22 has the variant motif CNP (green) (ref.sec NG_028006.1). 


\subsubsection{Patients with decapitated spermatozoa carried normal sequences of exons 10 and 11 of the gene Hook1 compared to the reference wild-type sequence}

The murine Hookl gene is highly expressed in testis. Hookl has 11 splice variants in mice and 6 in humans. The longest Hookl transcript in humans and mice comprises 22 exons. In mice, the deletion of exons 10 and 11 affects HOOK1 functionality and perturbs the correct attachment of sperm head and tail (Mendoza-Lujambio, 2002). In humans, a missense mutation affecting the exon 10 has been identified. This mutation localizes in the central region of the coiled-coil domain, where the base pair $\mathrm{G}$ is replaced by A, causing a non-synonymous change in p.Q286R (Chen et al., 2018). Therefore, an alteration in the coiled-coil motif might affect the protein's ability to interact with other proteins and bind to organelles. Besides that, HOOK1 localizes to the manchette in mouse spermatids and is involved in the interconnection between the manchette microtubules (Walenta et al., 2001). Thus, HOOK1 is implicated in the manchette's correct positioning and its proper connection to the nuclear envelope. Therefore, a dysfunctional protein might be responsible for decapitated spermatozoa. It proposes Hookl as a causative candidate gene for human teratozoospermia or decapitation syndrome (Mendoza-Lujambio, 2002).

I investigated exons 10 and 11 of the Hookl gene to figure out whether Hook1 is involved in the acephalic syndrome represented by these two patients. Exon 10 and 11 were amplified using the primer pairs Hook1-E10-N-for/Hook-Exon10-Rev and Hook1-E11-N-for/Hook1-E11-NRev, respectively (Tab. 6.1.1). Both exons were amplified and cloned into the vector pJET1.2/blunt (Fermentas). The DNA isolated from 2 up to 8 individual colonies were sequenced. Sequences of exon 10 and 11 from both patients exhibit the same nucleotide sequence as the reference NC_000001.11 (Fig. 3.5-3.6-3.7). Mutations were not found in the regions investigated, including the annotated deletion of exon 10 and 11 . Therefore, the possibility was excluded that mutations in exon 10 or 11 might be causative for the acephalic spermatozoan syndrome in the patients studied here. 


\begin{tabular}{|c|c|c|c|}
\hline Hook1_Ex10 & \multicolumn{2}{|c|}{ CAAGGCTTGAAGCTGCAAAAGATGATTACCGTGTTCACTGTGAAGAACTTGAAAAGCAGC } & 60 \\
\hline P17_ClonA2 & \multicolumn{2}{|c|}{ CAAGGCTTGAAGCTGCAAAAGATGATTACCGTGTTCACTGTGAAGAACTTGAAAAGCAGC } & 60 \\
\hline P17_ClonA3 & \multicolumn{2}{|c|}{ CAAGGCTTGAAGCTGCAAAAGATGATTACCGTGTTCACTGTGAAGAACTTGAAAAGCAGC } & 60 \\
\hline P17_ClonB4 & \multicolumn{2}{|c|}{ CAAGGCTTGAAGCTGCAAAAGATGATTACCGTGTTCACTGTGAAGAACTTGAAAAGCAGC } & 60 \\
\hline $\mathrm{P} 17^{-} \mathrm{Cl}$ ○nB6 & \multicolumn{2}{|c|}{ CAAGGCTTGAAGCTGCAAAAGATGATTACCGTGTTCACTGTGAAGAACTTGAAAAGCAGC } & 60 \\
\hline P17_Clong19 & \multicolumn{2}{|c|}{ CAAGGCTTGAAGCTGCAAAAGATGATTACCGTGTTCACTGTGAAGAACTTGAAAAGCAGC } & 60 \\
\hline $\mathrm{P} 17^{-} \mathrm{Clong} 21$ & \multicolumn{2}{|c|}{ CAAGGCTTGAAGCTGCAAAAGATGATTACCGTGTTCACTGTGAAGAACTTGAAAAGCAGC } & 60 \\
\hline $\mathrm{P} 17^{-} \mathrm{ClonH} 23$ & \multicolumn{2}{|c|}{ CAAGGCTTGAAGCTGCAAAAGATGATTACCGTGTTCACTGTGAAGAACTTGAAAAGCAGC } & 60 \\
\hline P17_ClonH24 & \multicolumn{3}{|c|}{ 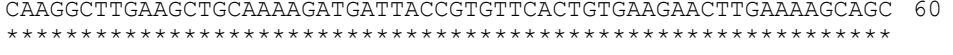 } \\
\hline Hook1_Ex10 & \multicolumn{2}{|c|}{ TAATCGAATTCCAGCATAGGAATGATGAATTGACTAGTCTTGCAGAAGAAACAAGAGCCC } & 120 \\
\hline P17_ClonA2 & \multicolumn{2}{|c|}{ TAATCGAATTCCAGCATAGGAATGATGAATTGACTAGTCTTGCAGAAGAAACAAGAGCCC } & 120 \\
\hline P17_ClonA3 & \multicolumn{2}{|c|}{ TAATCGAATTCCAGCATAGGAATGATGAATTGACTAGTCTTGCAGAAGAAACAAGAGCCC } & 120 \\
\hline $\mathrm{P} 17^{-} \mathrm{ClonB} 4$ & \multicolumn{2}{|c|}{ TAATCGAATTCCAGCATAGGAATGATGAATTGACTAGTCTTGCAGAAGAAACAAGAGCCC } & 120 \\
\hline P17 ClonB6 & \multicolumn{2}{|c|}{ TAATCGAATTCCAGCATAGGAATGATGAATTGACTAGTCTTGCAGAAGAAACAAGAGCCC } & 120 \\
\hline P17_ClonG19 & \multicolumn{2}{|c|}{ TAATCGAATTCCAGCATAGGAATGATGAATTGACTAGTCTTGCAGAAGAAACAAGAGCCC } & 120 \\
\hline P17_Clong21 & \multicolumn{2}{|c|}{ TAATCGAATTCCAGCATAGGAATGATGAATTGACTAGTCTTGCAGAAGAAACAAGAGCCC } & 120 \\
\hline P17_ClonH23 & \multicolumn{2}{|c|}{ TAATCGAATTCCAGCATAGGAATGATGAATTGACTAGTCTTGCAGAAGAAACAAGAGCCC } & 120 \\
\hline $\mathrm{P} 17_{-}^{-} \mathrm{ClonH} 24$ & \multicolumn{2}{|c|}{ 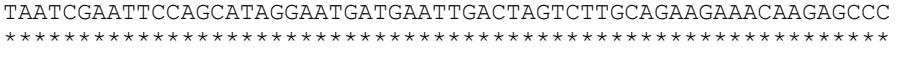 } & 120 \\
\hline Hook1 Ex10 & TGAAAGATGAAATAGATGTTCTTAG & 145 & \\
\hline P17_CIonA2 & TGAAAGATGAAATAGATGTTCTTAG & 145 & \\
\hline $\mathrm{P} 17^{-}$ClonA3 & TGAAAGATGAAATAGATGTTCTTAG & 145 & \\
\hline $\mathrm{P} 17^{-} \mathrm{C} 10 \mathrm{nB} 4$ & TGAAAGATGAAATAGATGTTCTTAG & 145 & \\
\hline P17_ClonB6 & TGAAAGATGAAATAGATGTTCTTAG & 145 & \\
\hline P17_ClonG19 & TGAAAGATGAAATAGATGTTCTTAG & 145 & \\
\hline P17_Clong21 & TGAAAGATGAAATAGATGTTCTTAG & 145 & \\
\hline P17_ClonH23 & TGAAAGATGAAATAGATGTTCTTAG & 145 & \\
\hline P17_ClonH24 & 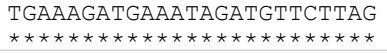 & 145 & \\
\hline
\end{tabular}

Figure 3.5. DNA sequence alignment of Hookl-exon10 from patient $\mathbf{n}^{\circ} \mathbf{1 7}$. The alignment of the eight clones analyzed are identical to the wild type sequence. Sequences were compared to the reference sequence NC_000001.11.

\begin{tabular}{|c|c|c|c|}
\hline Hook1_Ex10 & \multicolumn{2}{|c|}{ CAAGGCTTGAAGCTGCAAAAGATGATTACCGTGTTCACTGTGAAGAACTTGAAAAGCAGC } & 60 \\
\hline P19 ClonK1 & \multicolumn{2}{|c|}{ CAAGGCTTGAAGCTGCAAAAGATGATTACCGTGTTCACTGTGAAGAACTTGAAAAGCAGC } & 60 \\
\hline P19-Clonk2 & \multicolumn{2}{|c|}{ CAAGGCTTGAAGCTGCAAAAGATGATTACCGTGTTCACTGTGAAGAACTTGAAAAGCAGC } & 60 \\
\hline P19 ClonL6 & \multicolumn{2}{|c|}{ CAAGGCTTGAAGCTGCAAAAGATGATTACCGTGTTCACTGTGAAGAACTTGAAAAGCAGC } & 60 \\
\hline P19- Clonm7 & \multicolumn{2}{|c|}{ CAAGGCTTGAAGCTGCAAAAGATGATTACCGTGTTCACTGTGAAGAACTTGAAAAGCAGC } & 60 \\
\hline P19-ClonM8 & \multicolumn{2}{|c|}{ CAAGGCTTGAAGCTGCAAAAGATGATTACCGTGTTCACTGTGAAGAACTTGAAAAGCAGC } & 60 \\
\hline P19-ClonN10 & \multicolumn{2}{|c|}{ CAAGGCTTGAAGCTGCAAAAGATGATTACCGTGTTCACTGTGAAGAACTTGAAAAGCAGC } & 60 \\
\hline P19- ClonN11 & \multicolumn{2}{|c|}{ CAAGGCTTGAAGCTGCAAAAGATGATTACCGTGTTCACTGTGAAGAACTTGAAAAGCAGC } & 60 \\
\hline P19_ClonN12 & \multicolumn{3}{|c|}{ 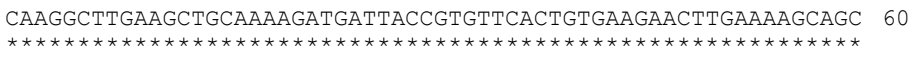 } \\
\hline Hook1_Ex10 & \multicolumn{2}{|c|}{ TAATCGAATTCCAGCATAGGAATGATGAATTGACTAGTCTTGCAGAAGAAACAAGAGCCC } & 120 \\
\hline P19 Cl̄onK1 & \multicolumn{2}{|c|}{ TAATCGAATTCCAGCATAGGAATGATGAATTGACTAGTCTTGCAGAAGAAACAAGAGCCC } & 120 \\
\hline P19- Clonk2 & \multicolumn{2}{|c|}{ TAATCGAATTCCAGCATAGGAATGATGAATTGACTAGTCTTGCAGAAGAAACAAGAGCCC } & 120 \\
\hline P19 ClonL6 & \multicolumn{2}{|c|}{ TAATCGAATTCCAGCATAGGAATGATGAATTGACTAGTCTTGCAGAAGAAACAAGAGCCC } & 120 \\
\hline P19 Clonm7 & \multicolumn{2}{|c|}{ TAATCGAATTCCAGCATAGGAATGATGAATTGACTAGTCTTGCAGAAGAAACAAGAGCCC } & 120 \\
\hline $\mathrm{P} 19^{-} \mathrm{ClonM} 8$ & \multicolumn{2}{|c|}{ TAATCGAATTCCAGCATAGGAATGATGAATTGACTAGTCTTGCAGAAGAAACAAGAGCCC } & 120 \\
\hline P19_ClonN10 & \multicolumn{2}{|c|}{ TAATCGAATTCCAGCATAGGAATGATGAATTGACTAGTCTTGCAGAAGAAACAAGAGCCC } & 120 \\
\hline P19- ClonN11 & \multicolumn{2}{|c|}{ TAATCGAATTCCAGCATAGGAATGATGAATTGACTAGTCTTGCAGAAGAAACAAGAGCCC } & 120 \\
\hline P19_ClonN12 & \multicolumn{2}{|c|}{ 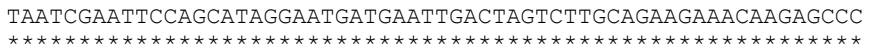 } & 120 \\
\hline Hook1 Ex10 & TGAAAGATGAAATAGATGTTCTTAG & 145 & \\
\hline P19_ClonK1 & TGAAAGATGAAATAGATGTTCTTAG & 145 & \\
\hline $\mathrm{P} 19^{-} \mathrm{Clonk} 2$ & TGAAAGATGAAATAGATGTTCTTAG & 145 & \\
\hline P19_ClonL6 & TGAAAGATGAAATAGATGTTCTTAG & 145 & \\
\hline P19-ClonM7 & TGAAAGATGAAATAGATGTTCTTAG & 145 & \\
\hline $\mathrm{P} 19^{-} \mathrm{ClonM} 8$ & TGAAAGATGAAATAGATGTTCTTAG & 145 & \\
\hline P19-ClonN10 & TGAAAGATGAAATAGATGTTCTTAG & 145 & \\
\hline P19-ClonN11 & TGAAAGATGAAATAGATGTTCTTAG & 145 & \\
\hline P19_ClonN12 & 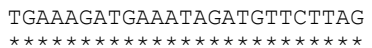 & 145 & \\
\hline
\end{tabular}

Figure 3.6. DNA sequence alignment of Hookl-exon10 from patient $\mathbf{n}^{\circ} 19$. The alignment of the eight clones analyzed are identical to the wild type sequence. Sequences were compared to the reference sequence NC_000001.11. 


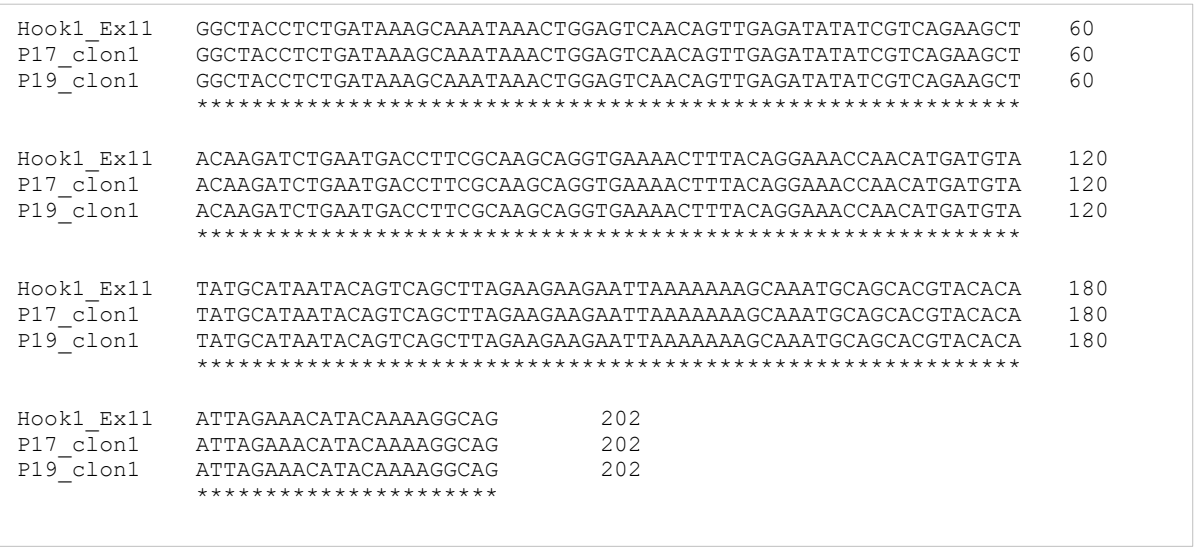

Figure 3.7. DNA sequence alignment of Hook1-exon11 from patient $\mathbf{n}^{\circ} \mathbf{1 7}$ and 19 . The alignment of the two clones analyzed are identical to the wild type sequence. Sequences were compared to the reference sequence NC_000001.11.

\subsubsection{Patients with decapitated spermatozoa carried normal sequences of exons 6 and 8 of the gene Spag4l compared to the reference wild-type sequence}

Spag41 consists of 13 exons in humans that encode the nuclear membrane protein SPAG4L/SUN5 of a size of $43 \mathrm{kDa}$. SUN5 localizes at the implantation fossa of mature spermatozoa (Yassine et al., 2015) and is essential for the attachment of the sperm head to the tail. Given that SPAG4L is an essential protein of the HTCA, I explored the sequences of exons 6 and 8, which have a size of $50 \mathrm{bp}$ and $109 \mathrm{bp}$, respectively.

Mutations of Spag4l in humans cause the abnormal attachment of the sperm flagellum to the nucleus. The disassembly is triggered by the absence of the implantation fossa and the basal plate. Previous analyses in patients with the acephalic spermatozoan syndrome have identified 11-point mutations. These mutations caused nonsense, missense, and one intron modification and can affect the protein structure (Zhu et al., 2016; Elkhatib et al., 2017), e.g., the mutations c.340G $\rightarrow$ A (p.Gly114Arg), c.425 + 1G $\rightarrow$ A (c.1043A>T) and c.475C $\rightarrow$ T (p.Arg159*) (Fig. 3.8).

The mutation c.340G $\rightarrow$ A (p.Gly114Arg) localizes in the predicted transmembrane domain, specifically to the last nucleotide of exon 5. According to a computational prediction, the replacement c.340G $\rightarrow$ A resulted in a missense mutation that affects the exon 5 and the expression of the SUN5 protein. Hence, the mutation c.340G $\rightarrow$ A might disrupt the localization of SUN5 at the nuclear envelope because of the impairment of the transmembrane domain (Zhu et al., 2016; Elkhatib et al., 2017; Shang et al., 2018). 
The intronic mutation c. $425+1 \mathrm{G} \rightarrow \mathrm{A}$ in humans localizes in the first base of intron 7 and introduces a premature stop codon. The induction of this mutation in the mouse testicular teratoma cell line F9 truncates the protein translation, which ends at Arg-142. Furthermore, this point mutation localizes upstream of the coiled-coil domain (Zhu et al., 2016; Elkhatib et al., 2017; Shang et al., 2018). Therefore, it might affect the protein conformation. According to a previous report, this protein alteration might disrupt the ability of SUN5 to interact with other proteins, especially with DNAJB13 (Shang et al., 2018).

A similar effect is caused by the mutation c. $475 \mathrm{C} \rightarrow \mathrm{T}$ (p.Arg159*). It localizes to exon 8 , inside the coiled-coil domain. This mutation introduces a premature stop codon and affects the secondary structure of the SUN5 protein. Furthermore, this premature protein termination increases the number of acephalic spermatozoa. Therefore, it has been suggested that this mutation disrupts the ability of SUN5 to interact with its counterparts (Shang et al., 2018).

Altogether, these data strongly indicate that impairments of the SUN5 function leads to the acephalic spermatozoa syndrome. Therefore, I analyzed different exons of the two unrelated infertile patients to identify possible mutations in Spag4l.

Exon 6 (50 bp length) was amplified using the primer pair Sun5-Ex7-For and Sun5-Ex7-Rev. This primer pair amplified a fragment of $200 \mathrm{bp}$, which includes part of the upstream and downstream introns. However, no mutations were found (Fig. 3.9 - 3.10-3.11). Thus, exon 6 seems not to be implicated in the acephalic spermatozoa syndrome in the two patients analyzed here. Exon 8 (109bp) was amplified using the primer pair suggested by Zhu et al. (2016), here named Sun5-Ex8-Zhu-For and Sun5-Ex8-Zhu-Rev. This primer pair amplified a fragment of $284 \mathrm{bp}$, which includes part of the upstream and downstream introns (Fig. 3.9). However, these primers turned out to be unspecific, amplifying a fragment of chromosome 17, whereas Spag4l localizes to chromosome 20. I also started to investigate exons 11 and 13 using the primer sequences specified by Zhu et al., Sun5-E11-Zhu-For/Sun5-E11-Zhu-Rev, and Sun5-E13-ZhuFor/Sun5-E13-Zhu-Rev. However, amplification of both exons was unsuccessful (Fig. 3.9). 


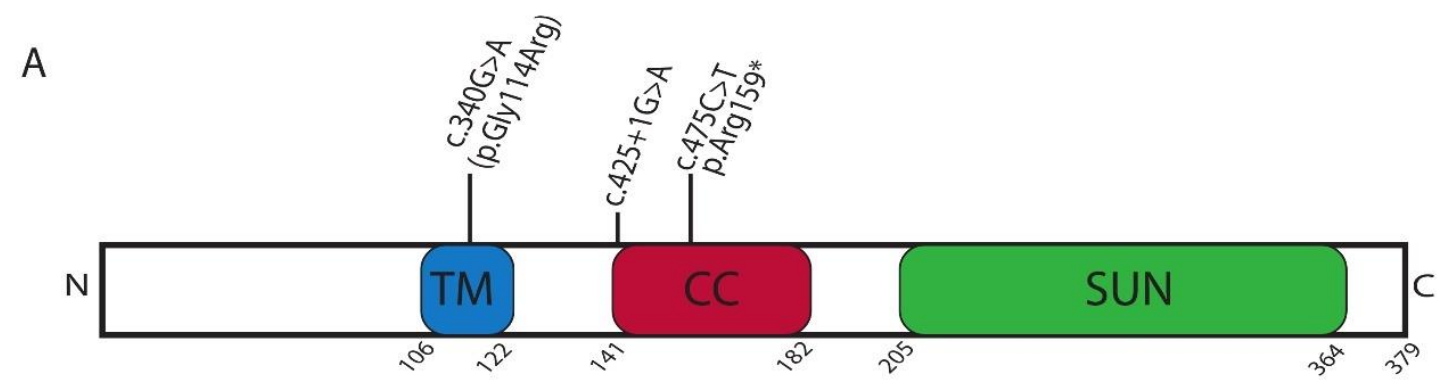

\begin{tabular}{|c|c|c|c|c|c|}
\hline B & Intron5 & D & Intron7 & $F$ & Exon8 \\
\hline WT & CTGTGCTTTdGGTGTTCCCA & WT & GCTTGAGGTAAGAGGAAT & WT & CAGGACCTCCGAGGTAG \\
\hline c. $340 \mathrm{G}>\mathrm{A}$ & $\begin{array}{l}\text { CTGTGCTTT ДAGTGTGTCCCA } \\
\text { c.340G>A (p.Gly114Arg) }\end{array}$ & c. $425+1 \mathrm{G}>\mathrm{A}$ & $\begin{array}{c}\text { GCTTGAGAfAAGAGGAAT } \\
\text { c. } 425+1 \mathrm{G}>\mathrm{A}\end{array}$ & $c .475 C>T$ & $\begin{array}{c}\text { CAGGACCTDTGAGGTAG } \\
\text { c.475C }>T\end{array}$ \\
\hline C & $\begin{array}{l}\text { Exon5 } \\
\end{array}$ & 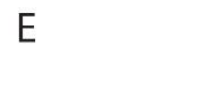 & Exon7 Exon8 & $\mathrm{G}$ & Exon8 \\
\hline $\begin{array}{l}\text { WT } \\
\text { c.340G }>A\end{array}$ & $\begin{array}{l}\text { MEKTGILLLCA GFWMSIHLPS } \\
\text { MEKTGILLLCAPRFWMFSIHLPS } \\
\text { c.340G>A (p.Gly114Arg) }\end{array}$ & $\begin{array}{l}\text { WT } \\
\text { c. } 425+1 G>A\end{array}$ & $\begin{array}{l}\text { PLQSLRLYQE } \\
\text { PLQSLR } * \text { YQE } \\
\text { c.425+1G>A }\end{array}$ & $\begin{array}{l}\text { WT } \\
\text { c.475C }>T\end{array}$ & $\begin{array}{l}\text { IQDURGSMNQLIAKLQE } \\
\text { IQDL*GSMNQLIAKLQE } \\
\text { c.475C>T }\end{array}$ \\
\hline
\end{tabular}

Figure 3.8. Mutations in TM and coiled-coil domain of the human Spag4l. (A) Distribution of mutations in Spag4l. c.340G>A affects the transmembrane domain (TM), c.425+1G>A situates upstream of the coiledcoil domain and c.475C $>\mathrm{T}$ situates in the coiled-coil domain. (B) c.340G $>\mathrm{A}$ mutation localizes in the last nucleotide of exon 5 (red square). (C) c.340G $>$ A mutation produces a missense change, p.Gly114Arg (red square). (D) c.425+1G $>\mathrm{A}$ situates in the first nucleotide of intron 7 (red square), introducing a premature stop codon (asterisk) and retaining partially $18 \mathrm{bp}$ of the intron 7. (E) c.425+1G>A produces a splicing-site mutation by the introduction of a stop codon (asterisk). (F) c. $475 \mathrm{C}>\mathrm{T}$ introduces a stop codon in exon 8 (red square and asterisk. $(\mathrm{G}) \mathrm{c} .475 \mathrm{C}>\mathrm{T}$ truncates the secondary structure of SPAG4L (asterisk). (Adapted from Zhu et al., 2016; Shang et al., 2018).

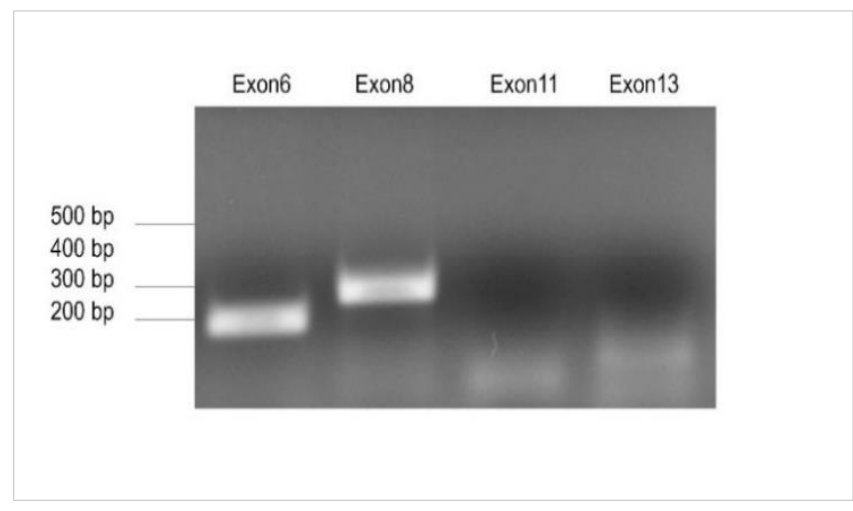

Figure 3.9. DNA amplification of exons 6, 8, 11, and 13 of Sun5. PCR was performed on DNA from patient $n^{\circ} 19$. Primers to amplify exon 6 generated the expected product size of $\sim 200$ bp (50 bp of exon $6+$ introns). Primers to amplify exon 8 generated a product of $\sim 300$ bp (109 bp of exon $8+$ introns) as expected. Primers to amplify exon 11 and 13 failed in the amplification. The expected product was not obtained. 


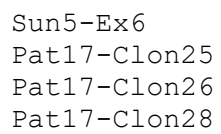

Figure 3.10. DNA sequence alignment of Sun5-exon6 from patient $\mathrm{n}^{\circ} \mathbf{1 7}$. The alignment of the three clones analyzed are identical to the wild type sequence. Sequences were compared to the reference sequence NG_054760.1.

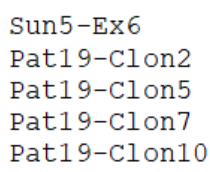

Figure 3.11. DNA sequence alignment of Sun5-exon6 from patient $\mathbf{n}^{\circ} 19$. The alignment of the four clones analyzed are identical to the wild type sequence. Sequences were compared to the reference sequence NG_054760.1.

\subsection{Characterization of the SUN-domain proteins SPAG4 and SPAG4L}

\subsubsection{Localization of the SUN domain proteins SPAG4L and SPAG4L2 at the nuclear membrane}

SPAG4 and SPAG4L play essential roles in the formation of the HTCA. The depletion of SPAG4 in mice weakens the attachment of the basal plate to the implantation fossa affecting the HTCA formation (Yang et al., 2018). Similarly, the partial disruption of Spag4l in mice interferes with the correct attachment of the sperm head to the tail (Shang et al., 2017). Additionally, the disruption of the exons that encode the SUN domain of SPAG4L results in acephalic spermatozoa by affecting the implantation fossa as observed in infertile men (Shang et al., 2017). Altogether, these data confirm the role of SPAG4 and SPAG4L in the formation of the head to the tail coupling apparatus. Therefore, the topological analysis of SPAG4L and its variant SPAG4L2 contributes to a better comprehension of their ability to recruit proteins to the nuclear envelope. Moreover, it could improve the understanding of the mechanisms to link the sperm head to the flagellum.

SPAG4L and SPAG4L2 are nuclear membrane proteins that share a similar structure. Both proteins are composed of one or two transmembrane domains in the $\mathrm{N}$-terminal region, 
respectively, a coiled-coil domain in the middle and a SUN domain in the C-terminal region. Spag4l and Spag4l2 are transcribed in testis, and their expression is correlated with the emergence of round spermatids during spermiogenesis (Xing et al., 2004; Frohnert et al., 2011).

Spag4l2 is a longer isoform of Spag4l that encodes two predicted transmembrane domains in its N-terminal region. In contrast, Spag 4 l encodes solely one. In both isoforms, transmembrane domains are followed by a coiled-coil region and the conserved SUN domain at the C-terminal (Xing et al., 2004; Frohnert et al., 2011). Moreover, SPAG4L2 has been described as nuclear membrane protein, with a $\mathrm{N}$-terminal region oriented towards the nucleoplasm, and a $\mathrm{C}$-terminal domain oriented towards the cytoplasm (Frohnert et al., 2011). Despite that fact, SPAG4L has been also identified as interacting partner of the cytoplamatic protein ODF1 (Shao et al., 2001). Given the localization of SPAG4L at the nuclear envelope (NE) it raises the question of how SPAG4L is able to interact with ODF1. Thus, I reevaluate the localization of SPAG4L2 domains and the impact of a truncated SUN-domain on the recruitment of the full-length protein to the NE.

To figure out in more detail the contribution of the diverse protein domains for the distribution of the whole protein, I generated a series of plasmids encoding specific parts of the protein fused to either the HA-, myc-, or EGFP-tag (Fig. 3.12). Plasmids were transfected into NIH3T3 cells, and their expression and protein localization were investigated by tag-specific antibodies.

Moreover, I investigated whether the disruption of the SUN domain affects the localization of SPAG4L2 at the nuclear membrane. It has been reported that a CRISPR-mediated disruption of the exons 10-12 encoding the SUN domain caused acephalic spermatozoa in mice (Shang et al., 2017). Thus, an expression plasmid encoding the truncated protein reported by Shang et al. (2017) was generated. 


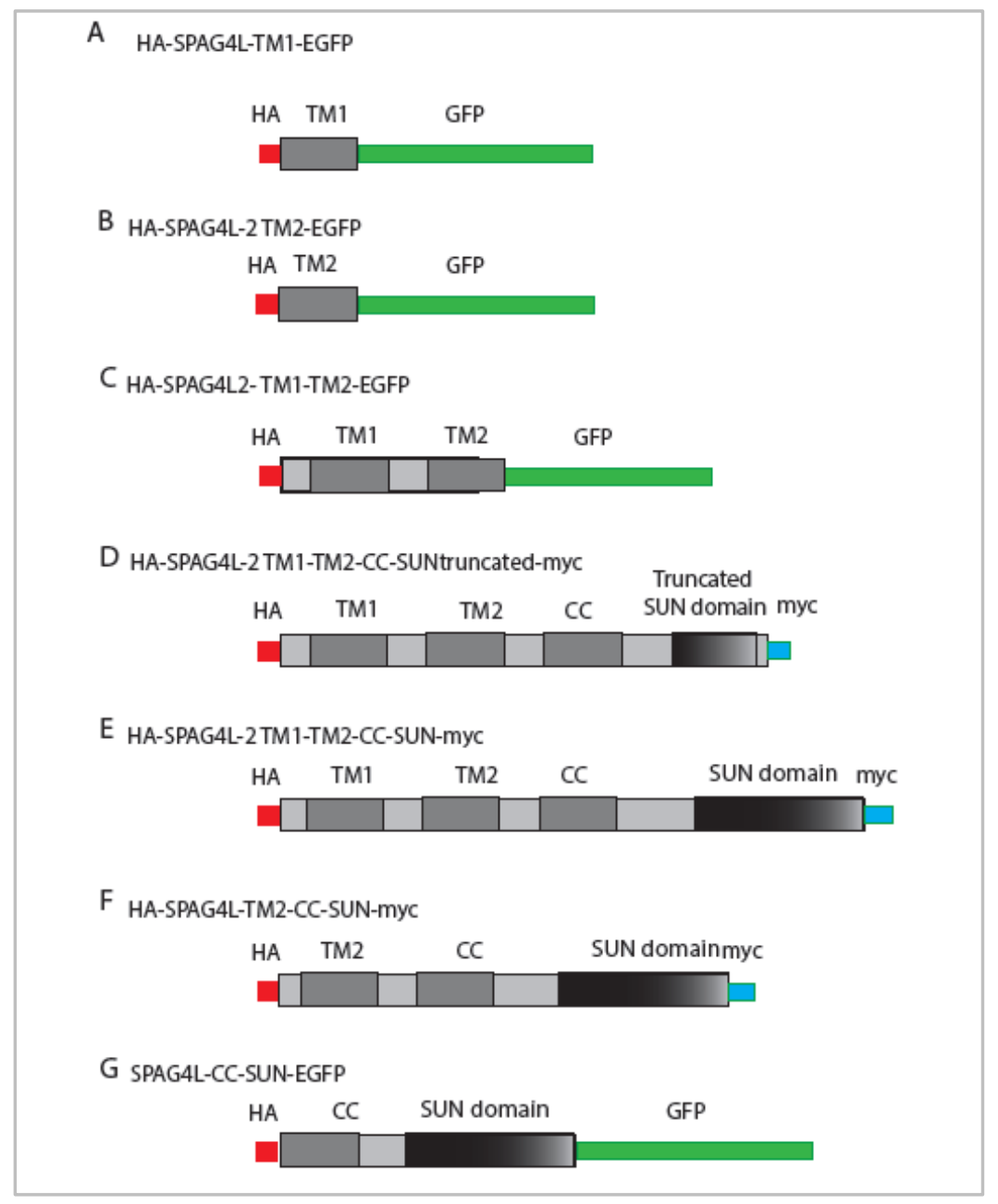

Figure 3.12. Schematic representation of Spag4l2 expression plasmids. Transmembrane domain 1 (TM1); transmembrane domain 2 TM2; coiled-coil domain (CC); SUN domain (SUN). (A) TM1 is tagged with HA at the N-terminal and GFP at the C-terminal. (B) TM2 is tagged with HA at the N-terminal and GFP at the Cterminal. (C) TM1 and TM2 tagged with HA at the N-terminal and GFP at the C-terminal. (D) Truncated Spag $4 l 2$ with a deletion of exon 10-12 encoding the SUN domain. Truncated Spag $4 l 2$ was tagged with HA at the N-terminal and with the myc epitope at the C-terminal. (E) Full-length Spag4l2 was tagged with HA at the $\mathrm{N}$-terminal and with the myc epitope at the C-terminal. (F) Full-length Spag4l, which contains only TM2, tagged with HA at the N-terminal and with the myc epitope at the C-terminal. (G) The Coiled-coil region and SUN domain were tagged with $\mathrm{HA}$ at the N-terminal and the EGFP epitope at the C-terminal.

Immunocytological analyses showed that the full-length SPAG4L2 protein localizes at the nuclear membrane as well as in the cytoplasm (Fig. 3.13 A-C). The TM1 tagged to HA at the $\mathrm{N}$-terminal and EGFP at the C-terminal is highly expressed in the cytoplasm and at the nuclear membrane (Fig. 3.13 D-G). In contrast, the TM2 tagged to HA at the N-terminal and GFP at the C-terminal was detected at the nuclear membrane and cytoplasm by its HA-tag. However, its EGFP-tag was only detected in the region that seems to be the endoplasmic reticulum. 
Additionally, the localization of the recombinant protein HA-TM2-EGFP was detected by its HA and EGFP tags in the region that corresponds to the Golgi apparatus endoplasmic reticulum by analogy to previous reports (Frohnert et al., 2011) (Fig. $3.13 \mathrm{H}-\mathrm{K}$ ). Expression of the combined TMs (TM1 and TM2), tagged with HA and EGFP, revealed a clear nuclear membrane localization (Fig. 3.13 L-O). Ectopic expression of the truncated Spag4l2, comprising TM1, TM2, and the coiled-coil region but without the SUN domain, demonstrated a clear nuclear membrane localization (Fig. 3.13 P-R). By contrast, the C-terminal region containing the coiledcoil region and the SUN domain showed only a cytoplasmatic distribution (Fig. 3.13 S-U). These results strongly indicate that the transmembrane domain I (TM1) is essential for SPAG4L2 localization at the nuclear envelope. Furthermore, the transmembrane domain II (TM2) seems not to be required for the localization of SPAG4L2 at the nuclear membrane. Moreover, the coiled-coil region and SUN-domain displayed a cytoplasmatic localization and they appear not to be responsible for SPAG4L recruitment to the nuclear envelope. 


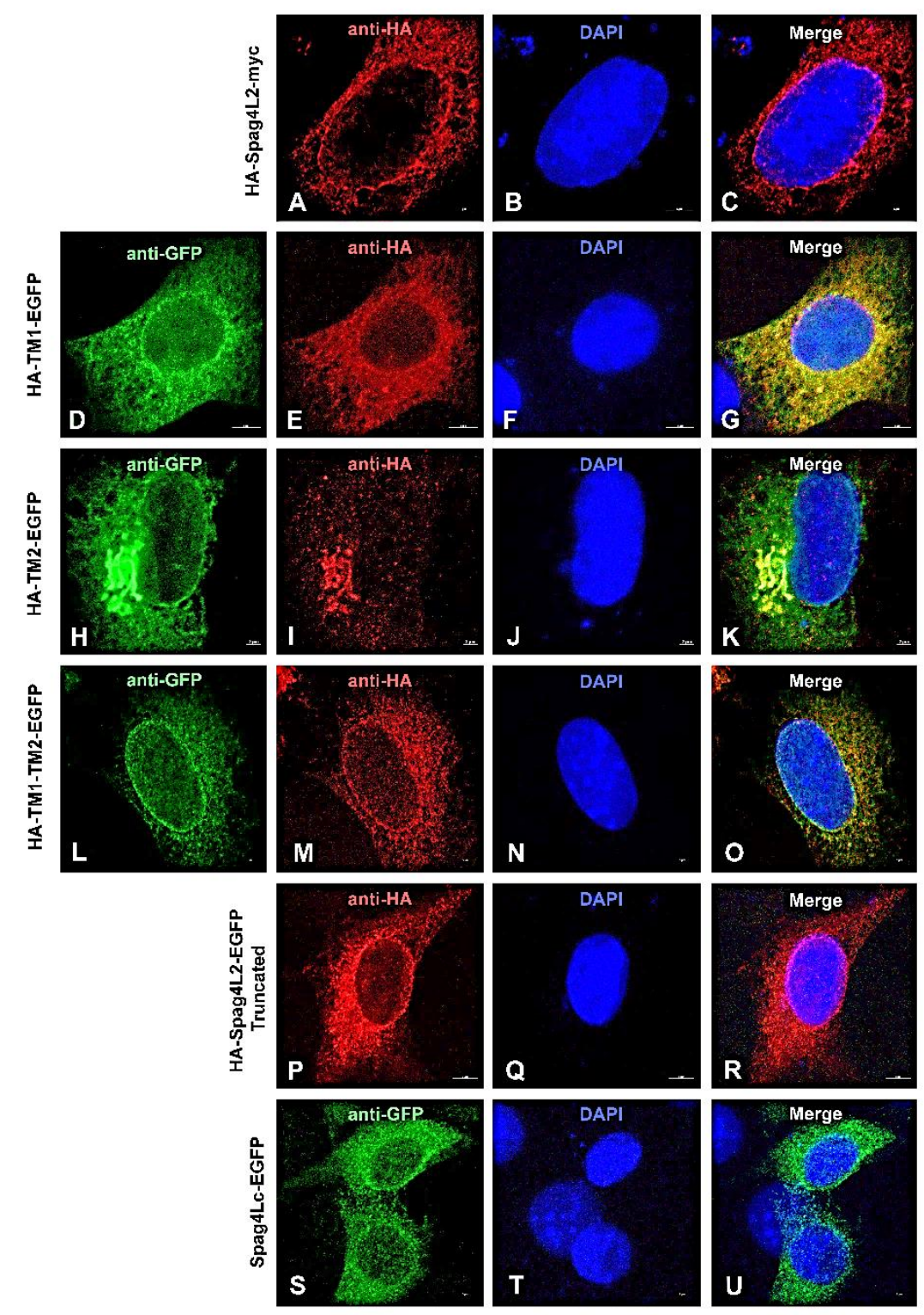

Figure 3.13. The protein domain organization of SPAG4L2 and their individual cellular distribution. Different expression plasmids were transfected into NIH3T3 cells and analyzed immunocytologically. (A-C) Full-length Spag4l2 (tagged with HA at the N-terminal and with the myc epitope at the C-terminal) localizes to the nuclear envelope and shows a mild distribution in the cytoplasm. (D-G) TM1 (tagged with HA at the $\mathrm{N}$-terminal and EGFP at the C-terminal) exposes a strong distribution in the cytoplasm and at the nuclear membrane. (H-K) TM2 (tagged with HA at the N-terminal and EGFP at the C-terminal) was detected at the nuclear membrane and the cytoplasm by its EGFP-tag, whereas the HA-tag was only detected in the cytoplasm. (L-O) TM1 and TM2 (tagged with HA at the N-terminal and EGFP at the C-terminal) expose a consistent localization at the nuclear envelope as well as a distribution to the cytoplasm. (P-R) The truncated SUN domain of Spag4l2 (tagged with HA at the N-terminal and with the myc epitope at the C-terminal) reveals a strong distribution in the cytoplasm and the nuclear membrane. (S-U) The Coiled-coil region and SUN domain (tagged with HA at the N-terminal and with the EGFP at the C-terminal) localizes in the cytoplasm. Nuclear counterstain with DAPI (blue). Bars are of $2 \mu \mathrm{m}$ (A-L) and $5 \mu \mathrm{m}$ (M-P). 


\subsubsection{Impact of Spag4- deficiency on gene expression during mouse spermatogenesis}

In mice, the expression of the Spag4 gene is restricted to testes, and its transcription starts in post-meiotic stages, around 18 days after birth. SUN4/SPAG4 is exclusively expressed in round and elongated spermatids. At the beginning of spermiogenesis, SPAG4 localizes at the nucleus of round spermatids, while in elongated spermatids, SPAG4 is situated close to the manchette. At the late stages of spermiogenesis, the manchette disassembles and SPAG4 is placed at the distal pole of the nuclear envelope. Here, it contributes to the linkage of head to tail in sperm (Yang et al., 2018). Thus, SPAG4 is essential for the correct sperm head shape, manchette organization, and anchorage of the sperm head to the flagellum (Calvi et al., 2015; Pasch et al., 2015; Yang et al., 2018).

SUN domain proteins reside in the nuclear envelope and participate in the linkage of the cytoskeleton to the nucleoskeleton (LINC) by their interaction with the conserved KASH domain proteins. Besides that, SUN domain proteins are involved in many cellular functions as telomere attachment to the inner nuclear membrane and chromosomes movements during meiosis (Malone et al., 1999; Padmakumar, 2005; Crisp et al., 2006; Ding et al., 2007; Razafsky \& Hodzic, 2009; Starr \& Fridolfsson, 2010).

In mice, spermatogenesis requires around 35 days (Oakberg, 1956). The process starts with the proliferation of spermatogonial stem cells (SSC). SSC undergo spermatogonia differentiation to produce A-, intermediate and B-spermatogonia. After the differentiation phase, Bspermatogonia produces pre-leptotene spermatocytes, which go through meiosis in $~ 8.6$ days old mice (Oakberg, 1956; Nebel et al., 1961; De Rooij \& Grootegoed, 1998; Eddy, 1998). Primary spermatocytes undergo the first meiotic division, including leptotene, zygotene, pachytene, diplotene. Once the first meiotic phase is completed, secondary spermatocytes experience a fast second meiotic division to produce round spermatids (RS) at 18 dpp. Finally, RS undergo spermiogenesis, which is completed after $30 \mathrm{dpp}$ and elongated spermatids are detected (Oakberg, 1956; Nebel et al., 1961; Clermont, 1972; Frank et al., 1986; Russell, 1990).

In the first step of spermatogenesis, the stimulated by retinoic acid gene (Stra8), a spermatogonial marker, is involved in spermatogonial proliferation and spermatocyte transition (Ma et al., 2018). Spermatocytes then enter meiosis and proteins as SYCP1 and SYCP3 regulate chromosome synapsis during zygotene-pachytene (Meuwissen et al., 1992; Yuan et al., 2000; Costa et al., 2005). Once round spermatids are produced, cells start the nuclear shaping and DNA packaging. During this process remodeling of the chromatin takes place that involves the replacement of somatic histones by testis-specific histones followed by transition proteins and 
finally by protamines (Balhorn, 1982). The male germ cell-specific expressed histone Hanp1/H1T2/H1-7 is one of the early replacement histones (Balhorn, 1982; Tanaka et al., 2005), whereas protamine 1 is first transcribed in round spermatids (Braun, 1990).

During spermiogenesis, two $\alpha$-tubulin isotypes restricted to testis, $M \alpha 3$ and $M \alpha 7$, start their transcription (Villasante et al., 1986). Odfl initiates its transcription in round spermatids before condensation and elongation of the spermatid nucleus (Burmester et al., 1996). Spag4 starts its transcription in postmeiotic spermatids (Pasch et al., 2015; Yang et al., 2018).

Given that SPAG4, a member of the SUN domain proteins, localizes at the nuclear membrane and interacts with proteins inside the nucleus, disruption of Spag4 might affect the transcription and spermatogenetic progression. Hence, to evaluate a possible influence of the deletion of Spag4 in male germ cells, I investigated the expression pattern of molecular marker genes that characterize the progression of spermatogenesis (Fig. 3.14). To achieve this purpose, RNA was isolated from adult testes of wild type mice as well as of heterozygous and homozygous Spag4deficient mice using animals aged 10-months (wild-type and Spag4 ${ }^{+/-}$\#319), 3-months

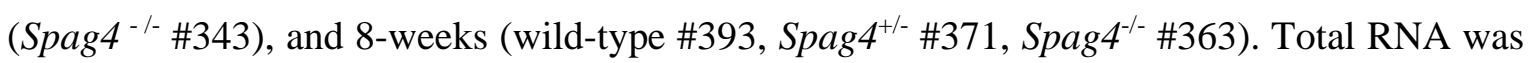
first treated with DNase and thereafter reverse transcribed into cDNA.

After the verification of the Spag4-genotype, the expression of meiotic and post-meiotic marker genes was investigated. Amplification of cDNA from wild-type versus Spag4-deficient mice showed a slight difference in Hanpl and Prm1 gene expression. Hanpl exhibits a weaker band in the amplified cDNA from Spag4 ${ }^{-/}$testis compared to wild-type samples, whereas Prml shows a stronger band in the amplification of the cDNA from Spag $4^{-/}$testis in comparison to wild-type (Fig. 3.15 A). Therefore, both genes were analyzed by qRT-PCR to quantify their expression.

First, specific primers were designed (Table 6.1.3), and their efficiencies and melting curves were tested by serial dilutions. The marker genes Stra8, Hanp1, Prm1, and Odf1, were quantified and their expression was calculated by the double delta $\mathrm{Ct}$ method. The housekeeping gene Hrpt was used as an internal reference gene (Fig. 3.15 B).

Two independent qRT-PCRs were performed. The first one included animals between 3 and 10 months old, whereas the second one included 8-weeks old animals. Thus, the experiment was performed using 2 biological replicates and 3 technical replicates. The results of the qRT-PCR expression analyses confirmed the previous results obtained by RT-PCR. These results suggest that Spag4-depletion affects the expression of the post-meiotic gene Hanpl and Prm1. It was 
observed that Spag4-deficiency reduced the expression of Hanpl and increased the expression of Prml (Fig. 3.15 B).

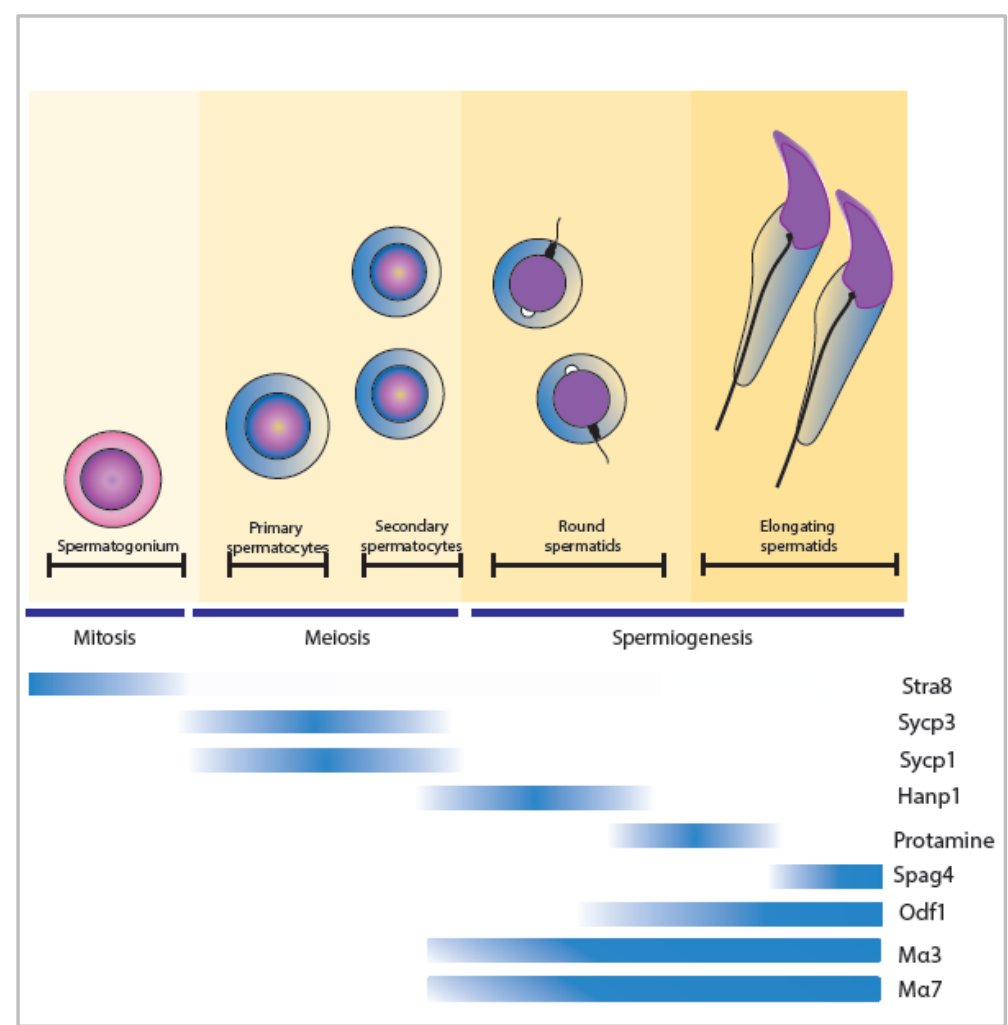

Figure 3.14. Schematic representation of the expression of gene markers during spermatogenesis. Spermatogonia are mitotically active cells that are characterized by the expression of Stra8. During meiosis, homologous chromosomes undergo synapsis. This process is assisted by synaptonemal complex proteins as SYCP1 and SYCP3. Chromatin remodeling starts in post-meiotic round spermatids with the replacement of somatic histones by testis-specific histones as HANP1. Afterward, histones are replaced by protamines. The transformation of round spermatids to mature spermatozoa includes the transcription of Spag4, Odf1, and the $\alpha$-tubulin isotypes $M \alpha 3$ and $M \alpha 7$. These genes encode essential proteins required for the head reshaping and the connection of the sperm head and tail. 


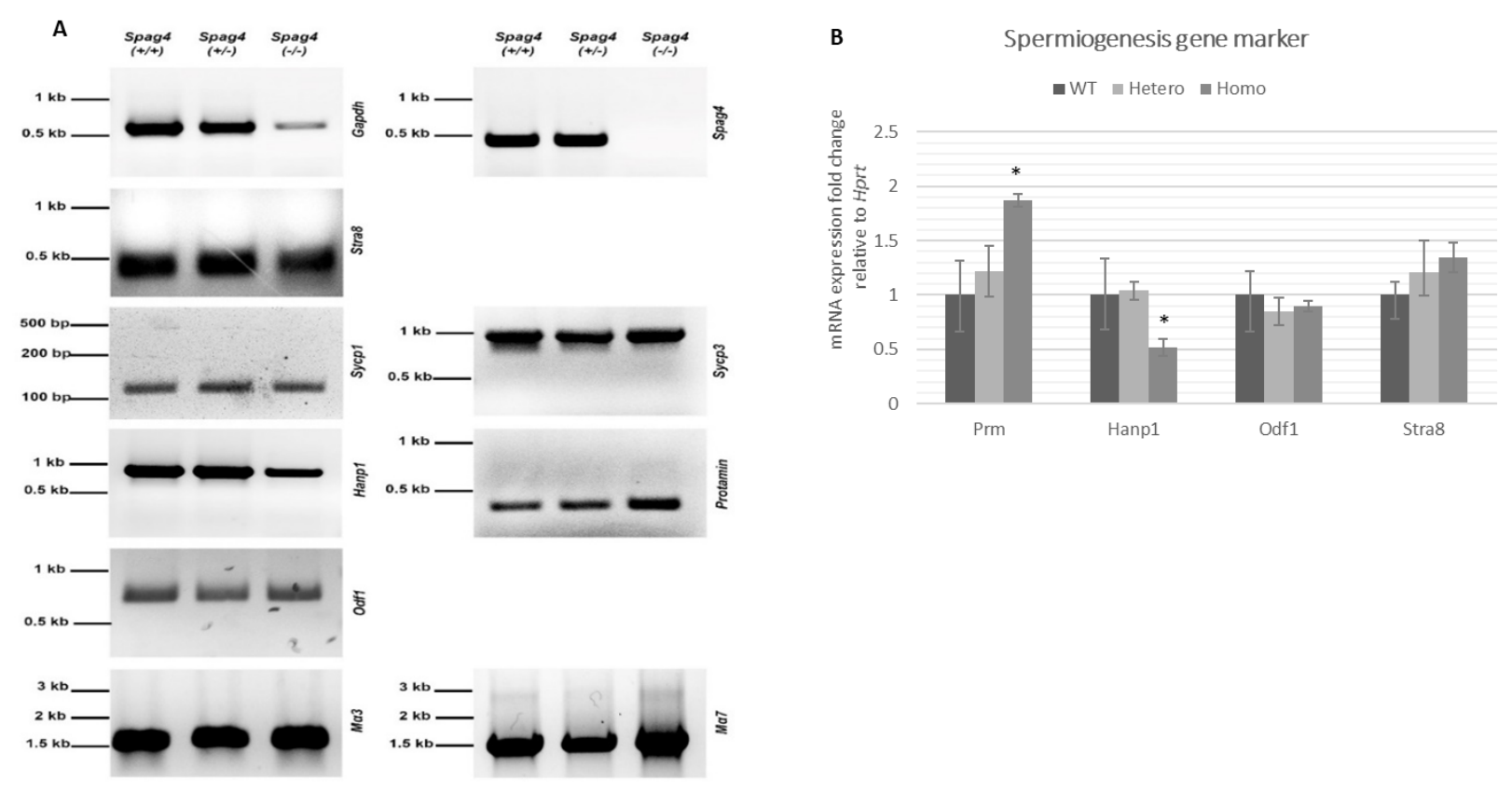

Figure 3.15. Gene expression profiling of marker genes for spermatogenic progression in Spag4deficient mice. (A) RT-PCR was performed on cDNA generated from testes of 8-weeks old mice. The correct genotype was verified by amplifying Spag4. Amplification of Gapdh was used to confirm cDNA quality. RTPCR shows normal expression of the premeiotic marker gene Stra8 and the meiotic marker genes Sycpl and Sycp3. The early post-meiotic marker gene Hanpl shows a reduced transcription in Spag4 $4^{--}$mice, whereas Prml increases its transcription. The late post-meiotic marker genes $O d f 1, M \alpha 3$, and $M \alpha 7$, display a homogeneous expression. (B) qRT-PCR analysis for gene markers during spermatogenesis. The expression of Prm1, Hanp1, Odf1 and Stra8 was evaluated using testicular cDNA obtained from wild type, Spag4 ${ }^{+/-}$and Spag $^{-/-}$mice. Relative expression values were calculated by normalizing to Hprt expression. Spag4-depletion caused increased expression of Prml and reduction of Hanpl expression. Significance is indicated with $* \mathrm{p} \leq 0.05$.

\subsubsection{The amber suppression method and its suitability for the identification of SPAG4 binding proteins}

Identifying novel SPAG4 bindings partners is essential for a better understanding of the sperm decapitation syndrome and male infertility to incerase our knowledge about the molecular components of the HTCA. Considering that SPAG4 is a SUN domain protein that mediates the linkage between the nucleoskeleton and the cytoskeleton via binding KASH domain proteins, it is important to investigate currently unknown KASH domain proteins and lamins that could be specific for the male germ cell (Starr \& Han, 2003; Crisp et al., 2006; Razafsky \& Hodzic, 2009). Identification of novel interaction partners by taking advantage of the native topology and orientation of SUN domain proteins inside the nuclear membrane is a challenging task but 
avoids severe artificial conditions that are present in Co-IP or pull-down assays (Choi et al., 2019). I, therefore, explored whether the amber suppression method is suitable for this purpose.

The amber suppression method takes advantage of an expansion of the genetic code. The genetic code comprises 64 three-base codons. Of these codons, 61 encode for 20 canonical amino acids, whereas the other 3 are the nonsense or termination codons (UAA-Ochre, UAG-Amber, and UGA-Opal). The genetic code was considered universal and "frozen" because it remained invariable in all organisms (Hinegardner and Engelberg, 1963; Woese et al., 1964; Sharp and Bulmer, 1988). However, in mitochondrial and nuclear genomes, the genetic code has evolved, e.g., in Mycoplasma sp., which translates UGA as Trp, and in Acetabularia acetabulum that reassigns the stop codons UAA and UAG to Gln (Osawa et al., 1992; Knight et al., 2001). These expansions of the genetic code enable the artificial incorporation of unnatural amino acids into proteins.

To incorporate unnatural amino acids (UAAs) into proteins and expand the genetic code of mammalian cells, new tRNA and its corresponding aminoacyl tRNA synthetase (tRNA-aaRS) are required (Xie \& Schultz, 2005). First, a stop codon should be incorporated into the proteincoding sequence of interest. Then, the corresponding tRNA anticodon must be engineered to be orthogonal to the aaRS. The aaRS must be modified to solely recognize the UAA and not any other natural amino acid. Next, the aminoacyl tRNA synthetase, tRNA, and construct of interest are introduced into cells. Once all components have been transfected and in the presence of the UAA, the aaRS covalently links the tRNA with the UAA. Then, tRNA charged with the anticodon recognizes the complementary three-base termination codon in the mRNA incorporating the UAA into the protein (Knight et al., 2001; Xie \& Schultz, 2005).

Among the stop codons, the amber codon (UAG) is not used frequently by organisms as E.coli $(9 \%)$ and yeast (23\%). Therefore, its incorporation into mRNA does not significantly perturb the host growth (Xie \& Schultz, 2005). Furthermore, the amber tRNA anticodon can be expressed in mammalian cells and can be aminoacylated by the mutant aaRS. Beyond that, it is also possible to incorporate a photoreactive amino acid into proteins. The incorporation of a photoreactive amino acid enables the identification of protein interacting partners that are near to the photo-labeled protein of interest. The $p$-benzoyl-L-phenylalanine ( $p \mathrm{Bpa})$ is an unnatural amino acid that contains a photoreactive side chain (Kauer et al., 1986; Xie et al., 2005). The benzophenone group of $p \mathrm{Bpa}$ is an efficient photo-crosslinker. It reacts with peptides close to the target protein, specifically with nearby $\mathrm{C}-\mathrm{H}$ bonds, when cells are exposed to UV light of $350-365 \mathrm{~nm}$. This reaction produces covalent cross-links between interacting proteins. $p$ Bpa can be incorporated in vivo into proteins using an orthogonal aminoacyl-tRNA 
synthetase/tRNA-pair that translates the amber codon (Kauer et al., 1986; Chin et al., 2003; Hino et al., 2005, Das and Oliver et al., 2011).

Thus, to first validate and explore the method, an orthogonal aminoacyl-tRNA synthetase/tRNA (p4XtRNA $p$ Bpa) that exclusively recognizes the amber stop codon, UAG, was generated. Furthermore, different plasmids encoding for lamin $\mathrm{C}$ were generated. Lamin $\mathrm{C}$ consists of an amino-terminal head, a helical rod domain (coiled-coil), and a carboxy-terminal tail domain. The C-terminal tail is highly conserved among lamins and adopts an immunoglobulin (Ig) fold (Dhe-Paganon et al., 2002; Krimm et al., 2002; Dittmer and Misteli 2011). Therefore, this region is supposed to be a hot spot for protein interactions. Thus, three different plasmids were designed in which amber codons were incorporated into the Ig-fold region of lamin C. An amber codon replaced the codons corresponding to Arg-470, Thr- 488, and Thr-534 in the coding region for the lamin $\mathrm{C}$ protein. The plasmids were named as pLMNC-R470-3X-FLAG, pLMNC-T488-3X-FLAG, and pLMNC-T534-3X-FLAG. Dr. Petra Neumann-Staubitz kindly provided all plasmids.

First, I evaluated the effective incorporation of the unnatural amino acid $p$ Bpa. NIH3T3 cells were transfected with plasmids that encode the orthogonal tRNA-aaRS (p4XtRNApBpa) and one of the plasmids containing the specific amber mutation. As expected, the orthogonal tRNAaaRS (p4XtRNA $p$ Bpa) incorporated $p$ Bpa into lamin $\mathrm{C}$ amino-acid sequence. Cells transfected with $\mathrm{p} 4 \mathrm{XtRNA} p \mathrm{Bpa} / p L M N C-R 470-3 X-F L A G, \mathrm{p} 4 \mathrm{XtRNA} p \mathrm{Bpa} / p L M N C-T 488-3 X-F L A G$, or p4XtRNA $p$ Bpa/pLMNC-T534-3X-FLAG were grown in media with or without supplementation of $p$ Bpa. After transfection, translation of LMNC comprising the amber codon was analyzed by immunofluorescence using the antibody anti-Flag.

The results revealed that the ectopic expression of pLMNC-R470-3X-Flag and p4XtRNApBpa in NIH3T3 cells showed an abnormal expression of LMNC. Lamins generally reside at the nuclear membrane; however, the genetically engineered LMNC localized inside the nucleus (Fig. $3.16 \mathrm{~A}-\mathrm{C}$ ). In contrast, supplementation of $1 \mathrm{mM}$ of $p$ Bpa in the cell growth media enables the correct translation of the protein and its location at the nuclear membrane (Fig. 3.16 D-F). Similar results were obtained when cells were transfected with the following combinations: either $\mathrm{p} 4 \mathrm{XtRNA} p \mathrm{Bpa} / p L M N C-T 488-3 X-F L A G$ or $\mathrm{p} 4 \mathrm{XtRNA} p \mathrm{Bpa} / p L M N C-T 534-3 X-F L A G$ (Fig. $3.16 \mathrm{G}-\mathrm{R}$ ). This experiment confirmed the efficient incorporation of the unnatural amino acid $p \mathrm{Bpa}$ into the genetically engineered LMNC sequence. The addition of $p \mathrm{Bpa}$ seemed neither to affect the protein sequence nor its localization at the nuclear membrane. 


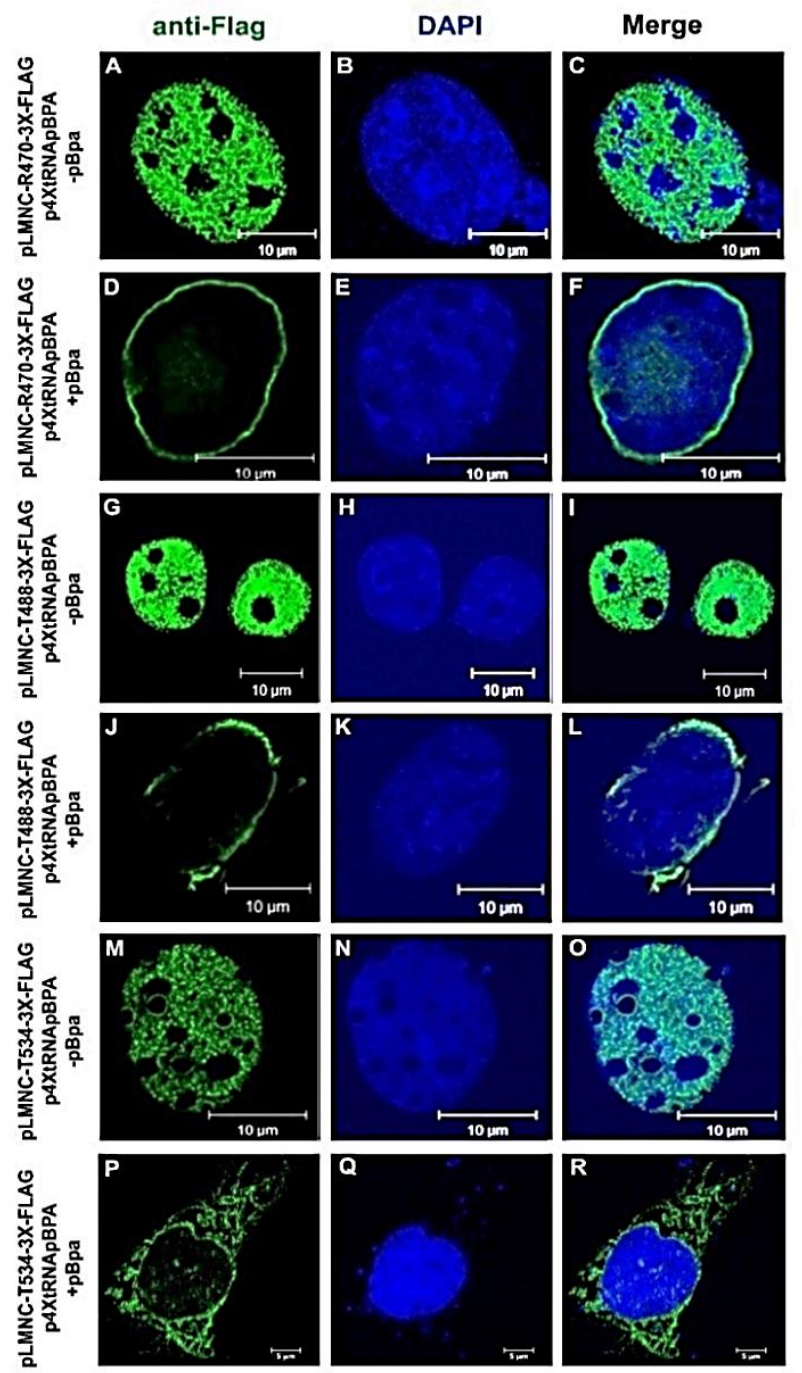

Figure 3.16 Incorporation of the unnatural amino acid pBpa into LMNC sequences. Three different Lmnc expression plasmids were co-transfected with the orthogonal tRNA-aaRS in NIH3T3 in the following order: (A-F) pLMNC-R470-3X-FLAG/ p4XtRNA $p$ Bpa, (GI) $\quad p L M N C-T 488-3 X-F L A G / \mathrm{p} 4 \mathrm{XtRNA} p \mathrm{Bpa}$, (M-R) pLMNC-T534-3X-FLAG/ p4XtRNA $p$ Bpa. NIH3T3 cells were either incubated in the absence of the unnatural amino acid $p$ Bpa (A-C; G-I; M-O) or with the addition of $p$ Bpa (D-F; J-L; P-R). Twenty-four hours post-transfection, cells were fixed and permeabilized. LMNC was detected using the anti-Flag antibody (green). Nuclei were stained using DAPI (blue). Scale bar, $10 \mu \mathrm{m}$ (A-O), and $5 \mu \mathrm{m}(\mathrm{P}-\mathrm{R})$.

In the next step, I attempted to reevaluate the interaction of the SUN domain proteins with LMNC. Previously, it has been reported that SUN1 interacts with lamin A (LMNA) but not with lamin C, B1, or B2 (Haque et al., 2006). Therefore, I expected that SPAG4 would not interact with LMNC either. The first experiment performed served as a negative control experiment to exclude any false positive interactions.

For this purpose, we used the plasmid HA-Spag4-MYC. HEK293 cells were transfected in triplicate with the orthogonal tRNA-aaRS (4XtRNApBpa) and HA-Spag4-MYC, and either one or the other Lmnc expression plasmid carrying the amber codon. One group of transfected cells was not treated with $p \mathrm{Bpa}$. The second and third groups were incubated with the unnatural amino acid $(p \mathrm{Bpa})$. Twenty-four hours after transfection, the third group of transfected cells (previously incubated with $p \mathrm{Bpa}$ ) was treated with UV-light of $\sim 365 \mathrm{~nm}$. In this case, UV-light acts as a linking agent causing covalent cross-linking of closely associated proteins. Proteins were isolated from all cells and analyzed by western blotting. 
To sum-up the experimental setting:

Group 1: $\quad$ HEK293 cells transfected with $p L M N C$-R470-3X-Flag, p4XtRNApBpa and HA-Spag4-MYC.

Group 2: $\quad$ HEK293 cells transfected with $p L M N C$-R470-3X-Flag, p4XtRNApBpa and HA-Spag4-MYC. Cells were incubated with $1 \mathrm{mM} p$ Bpa.

Group 3: $\quad$ HEK293 cells transfected with $p L M N C-R 470-3 X-F l a g$, p4XtRNA $p$ Bpa and HA-Spag4-MYC. Cells were incubated with $1 \mathrm{mM} p$ Bpa.

Exposition to $\sim 365 \mathrm{~nm}$ UV light

Negative control: Untreated HEK293 cells

The results are shown in Fig. 3.17. The first lane of the images A and B shows the proteins isolated and detected from group 1 of samples. The second lane corresponds to group 2 . The third lane corresponds to group 3, and lane four to untreated HEK293 cells. On the one hand, image A shows that HA-SPAG4-myc was successfully overexpressed in HEK293 cells. SPAG4 was detected using the mouse anti-HA antibody. The electrophoretic band was detected at $\sim 55$ $\mathrm{kDa}$, consistent with the expected molecular weight of SPAG4.

Further, SPAG4 was detected in the three different groups of transfected cells. On the other hand, several unspecific bands were detected by the antibody in the image-A. One of these bands is at $\sim 100 \mathrm{kDa}$ and it is present in all the samples, including the negative control (lane four). Moreover, there are protein bands at $\sim 130 \mathrm{kDa}$ and $\sim 180 \mathrm{kDa}$ in the first lane. These unspecific bands do not correspond to a potential cross-linked complex between SPAG4 and LMNC, which has an expected molecular mass of $\sim 110 \mathrm{kDa}$ corresponding to the sum of SPAG4 (55 kDa) and LMNC (62 kDa) (Fig. 3.16, A). To elucidate the origin of these unexpected protein bands, I transfected HEK293 cells with pLMNC-R470-3X-Flag, exclusively. However, the same unspecific protein bands were detected with the anti-HA antibody (data are not shown).

Regarding LMNC, the same protein lysates were immunoblotted and probed with mouse IgM anti-laminA/C antibody. The expected electrophoretic bands at $\sim 70 \mathrm{kDa}$ and $\sim 62 \mathrm{kDa}$, corresponding to LMNA and LMNC, respectively, were detected. This result was somehow unexpected because the third lane corresponds to proteins translated without $p$ Bpa supplementation. It was, therefore, expected that no recombinant LMNC would be generated (Fig. 3.17, B). The same protein lysates were immunoblotted with rabbit anti-Flag. However, the antibody detected unspecific bands even in the negative control (data are not shown). These data indicate that the endogenous expression of lamin A and lamin $\mathrm{C}$ seems to interfere with the 
detection of the recombinant LMNC. Besides that, no interaction between SPAG4 and LMNC was detected.

To sum-up, the incorporation of the unnatural amino acid $p$ Bpa into LMNC sequences was successful, as shown by immunocytological analyses. However, the detection of the genetically engineered LMNC protein by Western blotting was unsuccessful.
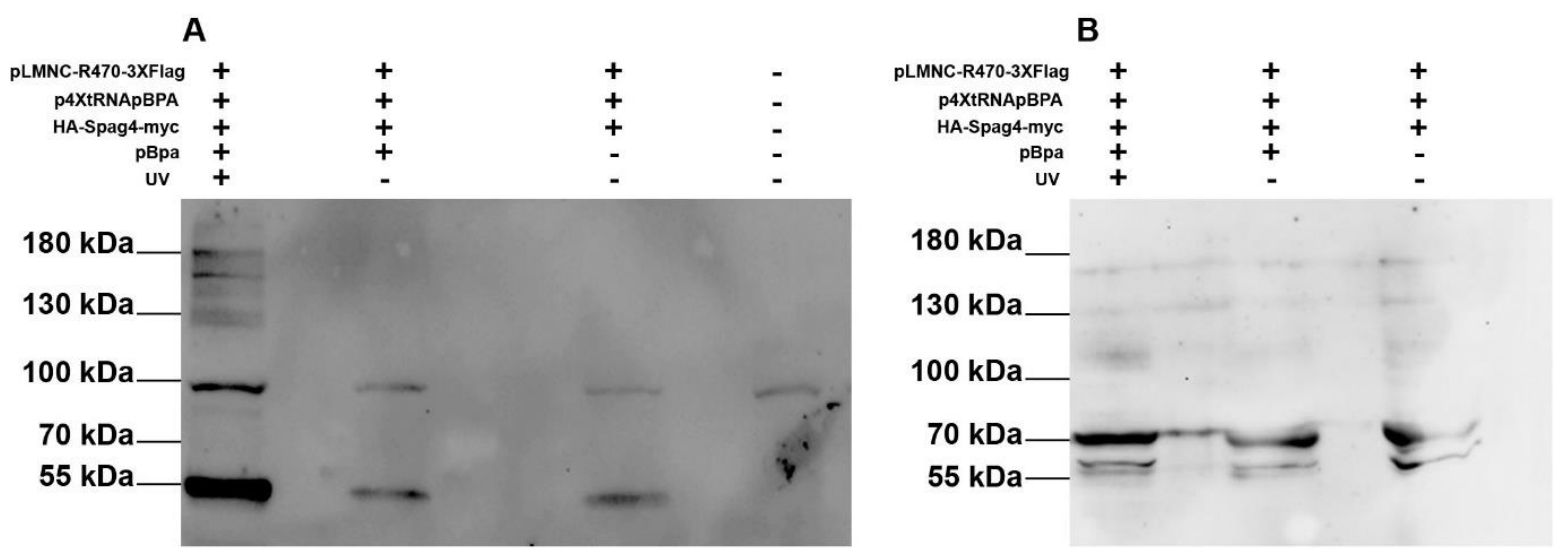

Figure 3.17 Evaluation of the amber suppression method by immunoblotting. HEK293 cells were transfected with $p L M N C-R 470-3 X-F L A G$, p4XtRNA $p$ Bpa, and HA-Spag4-MYC, followed by $p$ Bpa addition or exclusion and UV exposure or omission. Proteins were detected using either mouse anti-HA to identify HASpag4-MYC (A) or mouse IgM anti-laminA/C to identify LMNC (B). HA-Spag4-MYC was detected in all samples in the expected molecular mass of $\sim 55 \mathrm{kDa}$. Besides, an unspecific band was detected around 100 $\mathrm{kDa}$ (A). LaminA/C antibody detected two bands corresponding to LMNA of $\sim 70 \mathrm{kDa}$ and LMNC of $\sim 62$ $\mathrm{kDa}(\mathrm{B})$.

\subsubsection{Reevaluation of the topology of SPAG4 and SPAG4L across the nuclear envelope by drug-induced protein recruitment}

The SUN-domain proteins reside in the nuclear envelope. As anticipated from somatic SUNdomain proteins, their N-terminal region is located in the nucleus and their SUN domains in the perinuclear space (Crisp et al., 2006). The N-terminal contains up to two transmembrane domains, which recruit the full-length protein to the nuclear membrane. Beyond that, the orientation of testicular SUN-domain proteins, as e.g. SPAG4, is unknown. Considering that the C-terminal ends of SUN-domain proteins, including that of SPAG4 and SPAG4L, are inside the perinuclear space, it is completely unclear how they can directly interact with cytoskeletal proteins, as e.g. ODF1. Therefore, determining the topology of SPAG4 or SPAG4L in relation 
to the nuclear membrane requires a sophisticated analysis. To this end, I established the rapamycin system.

Rapamycin is a small molecule that binds to both FKBP and FRB to induce dimerization. In the absence of rapamycin, FKBP and FRB proteins do not interact (Fegan et al., 2010; Inobe \& Nukina, 2016). Thus, by the generation of fusion proteins that are either linked to FKBP or FRB, rapamycin induces their dimerization and, therefore, the recruitment of the fusion proteins.

The following sets of expression plasmids were designed to achieve this purpose: FRB-ECFPSUN4, FRB-ECFP-SUN5, Luciferase-mRFP-FKBP, pLCK-ECFP-FRB, and FKBP-mRFP. Here, it is important to remark that the N-terminal region of Sun4/Spag4 and Sun5/Spag4l were fused to the C-terminal region of FRB-ECFP. Given that the SUN-domain of SUN4 and SUN5 reside in the perinuclear space, their N-terminal coupled to FRB-ECFP could be oriented towards the nucleoplasm or to the cytoplasm. Thus, if the N-terminal region of FRB-ECFPSUN4 or FRB-ECFP-SUN5 is oriented towards the cytoplasm, FRB will be available to interact with its counterpart FKBP. This might indicate that the N-terminal of SUN4 and SUN5 might interact with cytoplasmic proteins, e.g. ODF1. It was expected that Luciferase-mRFP-FKBP localized in the cytoplasm and not in the nucleoplasm. The proteins Luciferase-mRFP-FKBP, pLCK-ECFP-FRB, and FKBP-mRFP were used as controls.

Plasmids were pairwise transfected into NIH3T3 cells:

a) FRB-ECFP-SUN4 and Luciferase-mRFP-FKBP: Luciferase-mRFP-FKBP should predominantly be expressed in the cytoplasm, whereas FRB-ECFP-SUN4 in the nuclear envelope. Rapamycin should enable the interaction of Luciferase-mRFP-FKBP and FRB-ECFP-SUN4, causing the recruitment of the Luciferase-mRFP-FKBP protein from the cytoplasm to the nuclear membrane.

b) FRB-ECFP-SUN5 and Luciferase-mRFP-FKBP: the localization and reaction of these proteins should be the same as in a).

c) FRB-ECFP-SUN4 and mRFP-FKBP: FRB-ECFP-SUN4 should be expressed in the nuclear membrane, whereas mRFP-FKBP should localize in both cytoplasm and nucleoplasm. Rapamycin should induce the dimerization and relocalization of mRFPFKBP to the nuclear membrane.

d) FRB-ECFP-SUN5 and $m R F P-F K B P$ : the localization and reaction of these proteins should be the same as described in a).

e) $p L C K-E C F P-F R B$ and Luciferase-mRFP-FKBP: Luciferase-mRFP-FKBP should mostly be expressed in the cytoplasm, whereas pLCK-ECFP-FRB should localize in 
both cytoplasm and nucleoplasm. Rapamycin may induce the relocalization of pLCKECFP-FRB from the nucleoplasm to the cytoplasm (control).

f) $\quad p L C K-E C F P-F R B$ and $m R F P-F K B P$ : Control. Both proteins should be expressed in the cytoplasm and nucleoplasm. Rapamycin addition should induce dimerization of both proteins but without changing their localization (control).

Rapamycin-induced heterodimerization of FKBP-rapamycin-FRB was started 24 hrs posttransfection by addition of $1 \mathrm{mM}$ rapamycin for 20 minutes.

Since SUN4 and SUN5 are nuclear envelope proteins, I expected that the recombinant proteins FRB-ECFP-SUN4 and FRB-ECFP-SUN5 would be likewise localized in the nuclear membrane. In contrast, Luciferase-mRFP-FKBP was expected to be cytoplasmic. Rapamycininduced heterodimerization between Luciferase-mRFP-FKBP and the FRB-fusion protein, either FRB-ECFP-SUN4 or FRB-ECFP-SUN5, should cause a re-localization and recruitment of Luciferase-mRFP-FKBP to the nuclear membrane (Fig. 3.18).

As expected, FRB-ECFP-SUN4 localizes at the nuclear membrane, whereas Luciferase-mRFPFKBP resides in the cytoplasm (Fig.3.19 A-H). The addition of rapamycin triggered the relocalization of Luciferase-mRFP-FKBP from the cytoplasm to the nuclear membrane which is visible by the red fluorescent rim at the nuclear membrane (Fig. 3.19 I-P).

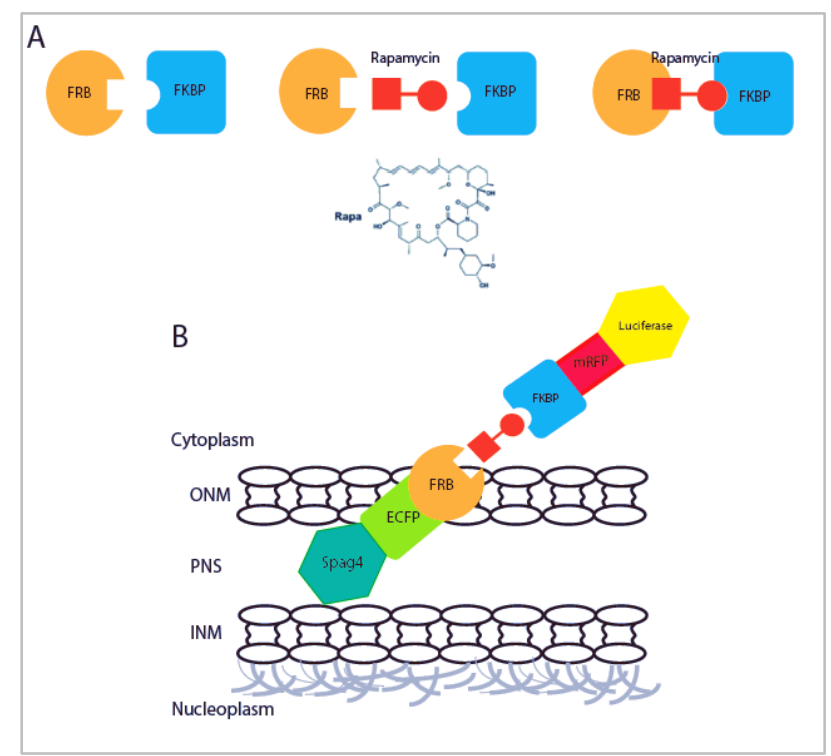

Figure 3.18. Schematic representation of the rapamycin system. (A) The interaction between FRB and FKBP is mediated by rapamycin. (B) FRB-ECFP-SUN4 resides in the nuclear envelope, whereas LuciferasemRFP-FKBP localizes in the cytoplasm. Rapamycin induces the relocation of Luciferase- mRFP-FKBP to the nuclear membrane by affinity binding between FKBP-Rapamycin-FRB. 
Luciferase-mRFP-FKBP is highly concentrated in the cytoplasm and at the area that seems to be the endoplasmic reticulum. The ER is an extension of the outer nuclear membrane (Lee et al., 1988). Therefore, it is difficult to clearly identify whether the proteins studied localize in the ER or at the nuclear membrane. Hence to inhibit protein translation, cells were incubated with puromycin for 4 hours before adding rapamycin. Thus, I expected that nuclear proteins would be recruited to the NE and would not be associated with the ER. However, cells treated with puromycin did not show considerable changes compared to the untreated cells (Fig. 3.19 Q- X).

Similar results were obtained by co-transfection of FRB-ECFP-Sun5 and Luciferase-mRFP$F K B P$. SUN5/SPAG4L resides at the nuclear membrane, while Luciferase-mRFP-FKBP is mostly localized in the cytoplasm without rapamycin incubation (Fig. 3.20 A-H). In contrast, rapamycin addition induces FKBP and FRB interaction, and hence the recruitment of Luciferase-mRFP-FKBP to the nuclear membrane via FRB-ECFP-SUN5 (Fig. 3.20 I-P).

As is known for the topology of the somatic SUN-domain proteins, SUN1 and SUN2, their Nterminal regions span the inner nuclear membrane and directly interact with lamin A and B (Hodzic et al., 2004; Padmakumar, 2005). Accordingly, the N-terminus is orientated towards the nucleoplasm. Therefore, I expected that the N-terminal fusion part FBR-ECFP linked to the N-terminal end of either SUN4 or SUN5 would be likewise exposed to the nucleoplasm and therefore not available for the rapamycin-induced dimerization when the FKBP-containing fusion protein localizes to the cytoplasm. However, the results demonstrate the ability of FRBECFP-SUN4 and FRB-ECFP-SUN5 to recruit Luciferase-mRFP-FKBP to the nuclear envelope, suggesting that the $\mathrm{N}$-terminal end localizes in the cytoplasm.

To further validate the results, two control plasmids were used, mRFP-FKBP and pLCK-ECFPFRB. Both plasmids encode fusion proteins of $\sim 50 \mathrm{kDa}$, that are, due to their small size, able to passively diffuse through the nuclear envelope and enter the nucleus (Paine et al. 1972). Thus, mRFP-FKBP should be easily recruited by FRB-ECFP-SUN4 or FRB-ECFP-SUN5 to the inner nuclear membrane in the presence of rapamycin. FRB-ECFP-Sun4 and FRB-ECFP-Sun5 were each co-transfected with $m R F P-F K B P$. FRB-ECFP-SUN4 localized at the nuclear membrane, whereas mRFP-FKBP has a strong expression in the nucleus (Fig. 3.21 A-D, I-L). The addition of rapamycin caused recruitment of mRFP-FKBP to the nuclear membrane by both, either FRBECFP-SUN4 as well as FRB-ECFP-SUN5. However, a substantial amount of mRFP-FRB remained in the nucleoplasm and the cytoplasm (Fig. 3.21 E-H; M-P). These results demonstrate that the N-terminal ends of SUN4 or SUN5, fused to the FRB-ECFP, are exposed to the nucleoplasm, and can therefore recruit mRFP-FKBP. 
Additionally, the cellular distribution of the recombinant proteins pLCK-ECFP-FRB and mRFP-FKBP were used as controls. Due to their small size, both proteins can pass the nuclear membrane and are therefore found in the nucleoplasm (Fig.3.21 Q-T). These data support the view that the luciferase fusion protein Luciferase-mRFP-FKBP localizes mainly in the cytoplasm. To finally demonstrate the rapamycin-induced heterodimerization, Luciferase$m R F P-F K B P$ was co-transfected with $p L C K$-ECFP-FRB into NIH3T3 cells. The addition of Rapamycin induced heterodimerization visible by the strong cytoplasmatic fluorescence (Fig.3.21 U-X).
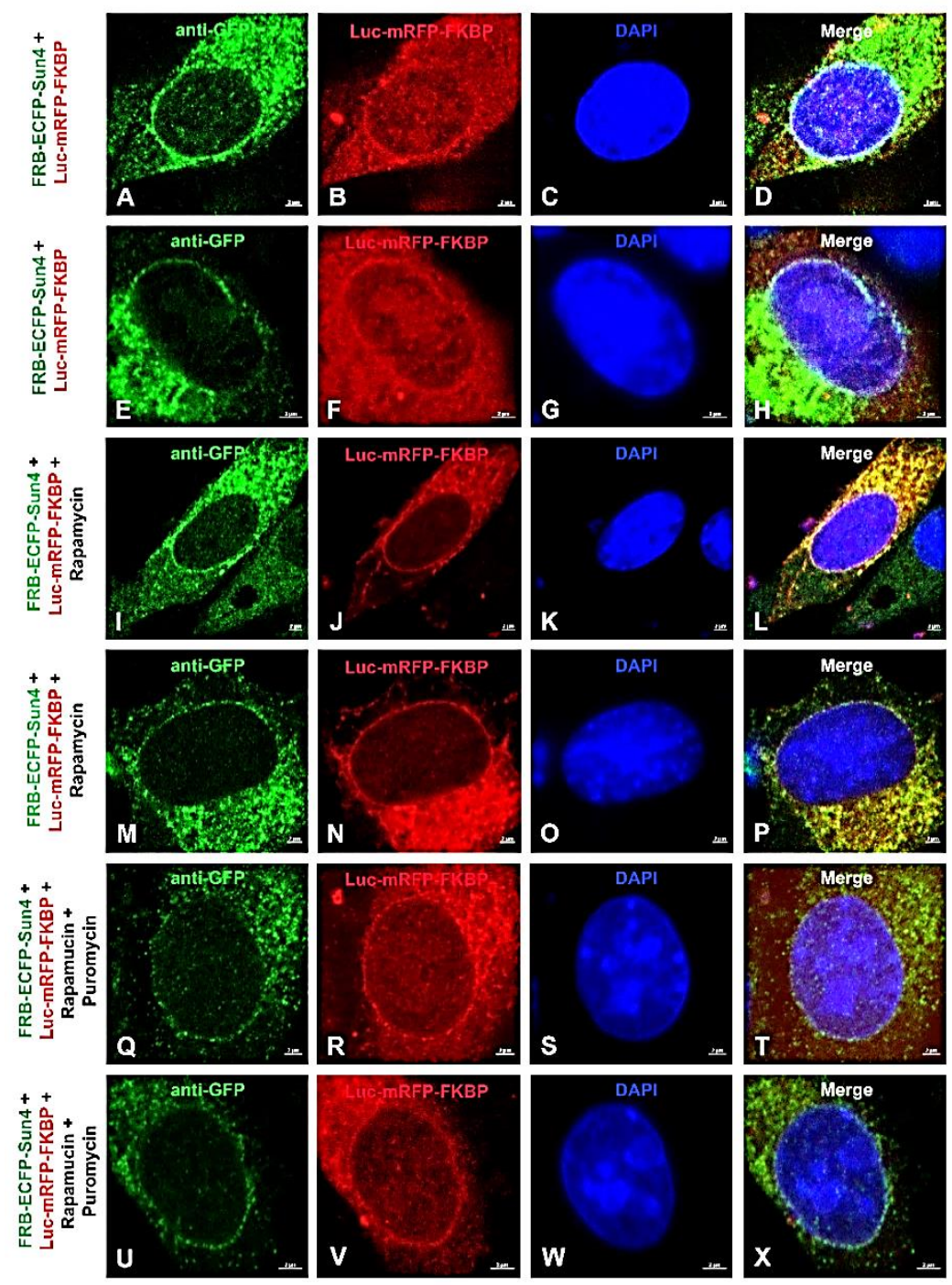

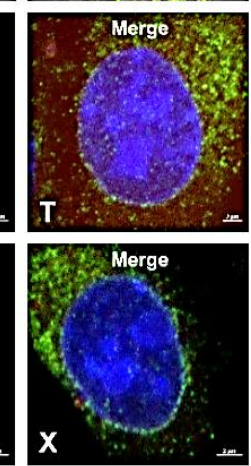

Figure 3.19. Rapamycin-induced recruitment of Luciferase-mRFP-FKBP to the nuclear envelope via FRB-ECFP-SUN4. Luciferase-mRFP-FKBP and FRB-ECFP-Sun4 were co-transfected into NIH3T3 cells and analyzed by immuno-cytology. FRB-ECFP-SUN4 was immunodecorated by anti-GFP antibody (green), and Luciferase-mRFP-FKBP was detected by its fluorescent tag (Luciferase-mRFP FKBP, red). (A-H) FRBECFP-SUN4 is located at the nuclear envelope, whereas Luciferase-mRFP-FKBP is found mostly in the 
cytoplasm and weakly in the nucleoplasm. (I-P) The addition of rapamycin induces the recruitment of Luciferase-mRFP-FKBP to the nuclear membrane resulting in a distinct red rim (Q-X). Pre-incubation with puromycin only weakened the cytoplasmic distribution of Luciferase-mRFP-FKBP and FRB-ECFP-SUN4. Nuclear counterstain with DAPI (blue). Bars are of $2 \mu \mathrm{m}$.

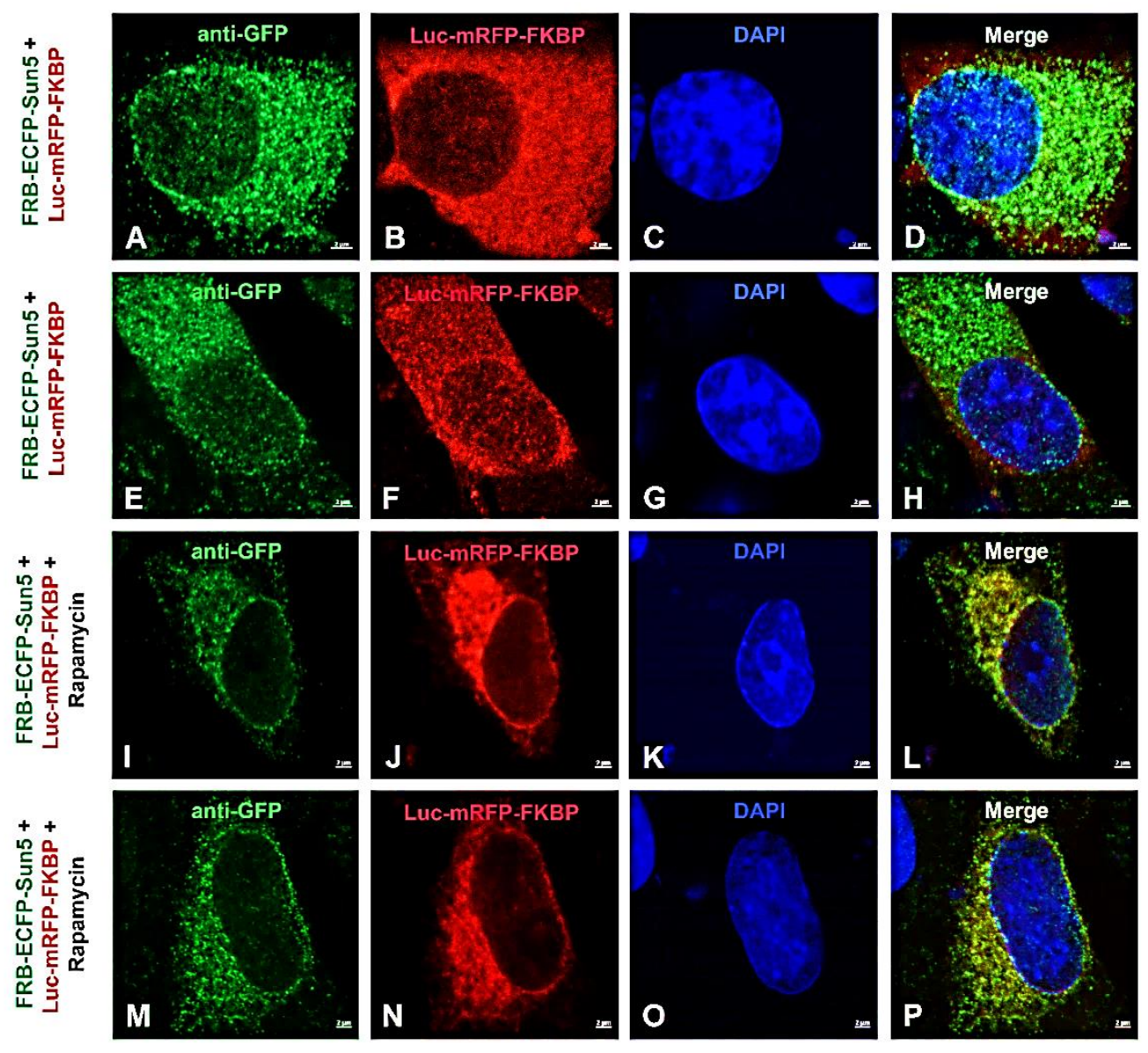

Figure 3.20. Rapamycin-induced recruitment of Luciferase-mRFP-FKBP to the nuclear envelope via FRB-ECFP-SPAG4L/SUN5. Luciferase-mRFP-FKBP and FRB-ECFP-Sun5 were co-transfected into NIH3T3 cells and analyzed by immuno-cytology. FRB-ECFP-SUN5 was immunodecorated with anti-GFP antibody (green), and Luciferase-mRFP-FKBP was detected by its red fluorescent tag (Luciferase-mRFPFKBP, red). (A-H) FRB-ECFP-SUN5 is located at the nuclear envelope without rapamycin, whereas Luciferase-mRFP-FKBP is mostly cytoplasmic. (I-P) The addition of rapamycin induces the re-localization of Luciferase-mRFP-FKBP to the nuclear membrane by heterodimerization of FRB-rapamycin-FKBP. Nuclear counterstain with DAPI (blue). Bars are of $2 \mu \mathrm{m}$. 

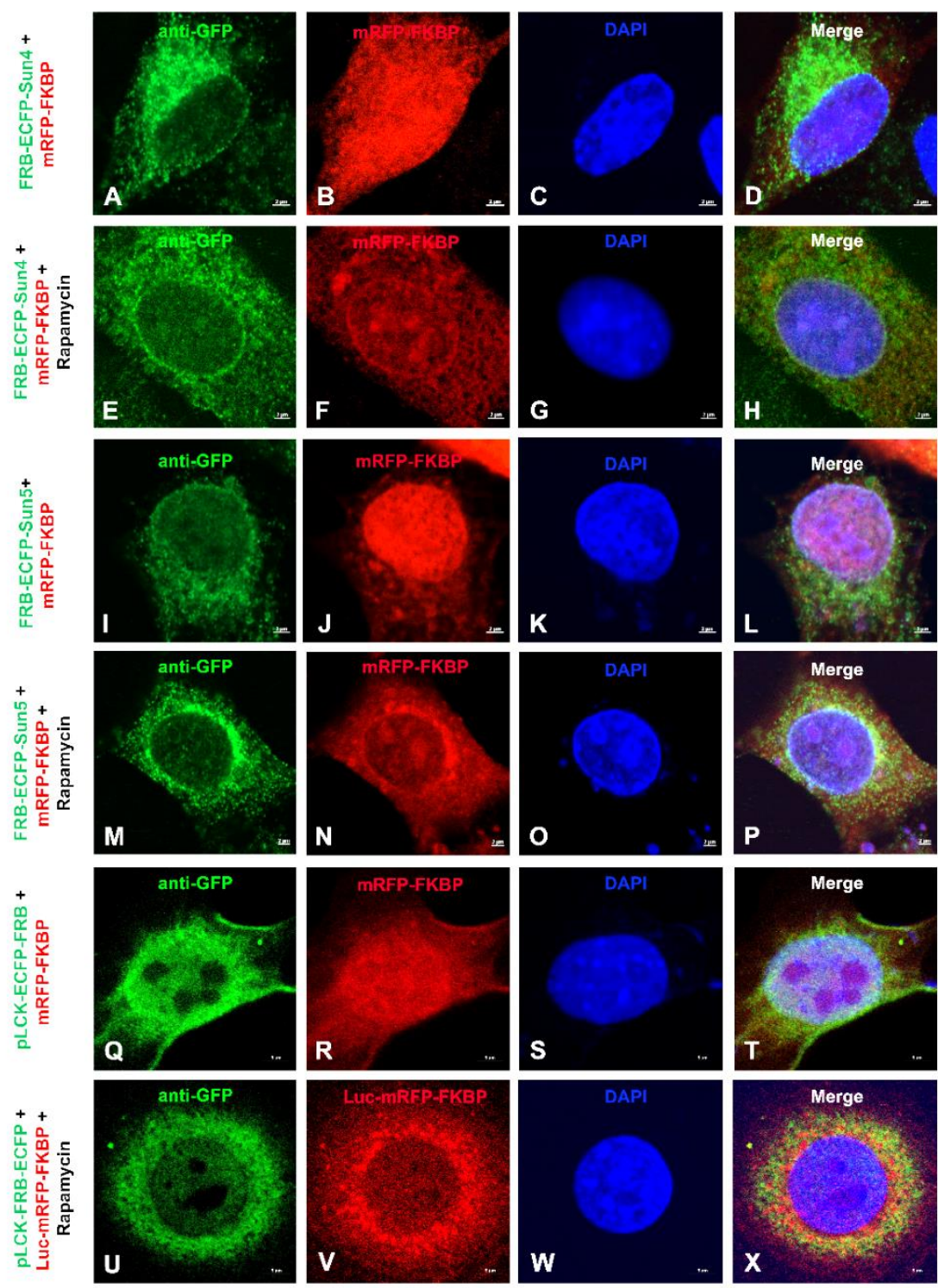

Figure 3.21. Controls for the Rapamycin system. FRB-ECFP-SUN4, FRB-ECFP-SUN5 and pLCK-FRBECFP were immunodecorated with anti-GFP antibody (green). Luciferase-mRFP-FKBP was detected by its red fluorescent protein, mRFP (red). (A-H) Co-expression of FRB-ECFP-Sun4 and $m R F P-F K B P$. (A-D) FRBECFP-SUN4 localizes at the nuclear envelope, whereas mRFP-FKBP is strongly expressed in the nucleoplasm. (E-H) Rapamycin addition induces the heterodimerization of FRB with FKBP recruiting mRFPFKBP to the nuclear membrane. (I-P) FRB-ECFP-SUN5 and mRFP-FKBP were co-transfected into NIH3T3 cells. (I-L) FRB-ECFP-SUN5 resides at the nuclear membrane, whereas mRFP-FKBP has a high expression in the nucleus. (M-P) The addition of rapamycin induces mRFP-FKBP recruitment to the nuclear membrane. (Q-X) Co-expression of $p L C K-E C F P-F R B$ and Luciferase-mRFP-FKBP. (Q-T) Cytoplasmic and nuclear localization of Luciferase-mRFP-FKBP and pLCK-ECFP-FRB in the absence of rapamycin. (U-X) Rapamycin induces the heterodimerization of both proteins in the cytoplasm. Nuclear counterstain with DAPI (blue). Bars are of $2 \mu \mathrm{m}$. 


\subsection{Generation of Spag4l/Sun5 knock out mice}

\subsubsection{Genotyping of ES cells}

To investigate the function of the Sun 5 gene, the project attempted to generate Sun 5 deficient mice using the knockout strategy. Homologous recombinant ES cells (Sun5tm1(KOMP)Vlcg, KOMP/Velocigene project: VG16078) were obtained from Velocigene Regeneron Pharmaceuticals Inc. The gene Spag4L/Sun5 was completely disrupted and replaced by the reporter gene LacZ and the resistant gene Neomycin (Fig. 3.22). Recombinant embryonic stem cells were evaluated by PCR and qPCR, according to the method described in 2.2.5.4 and 2.2.5.5.

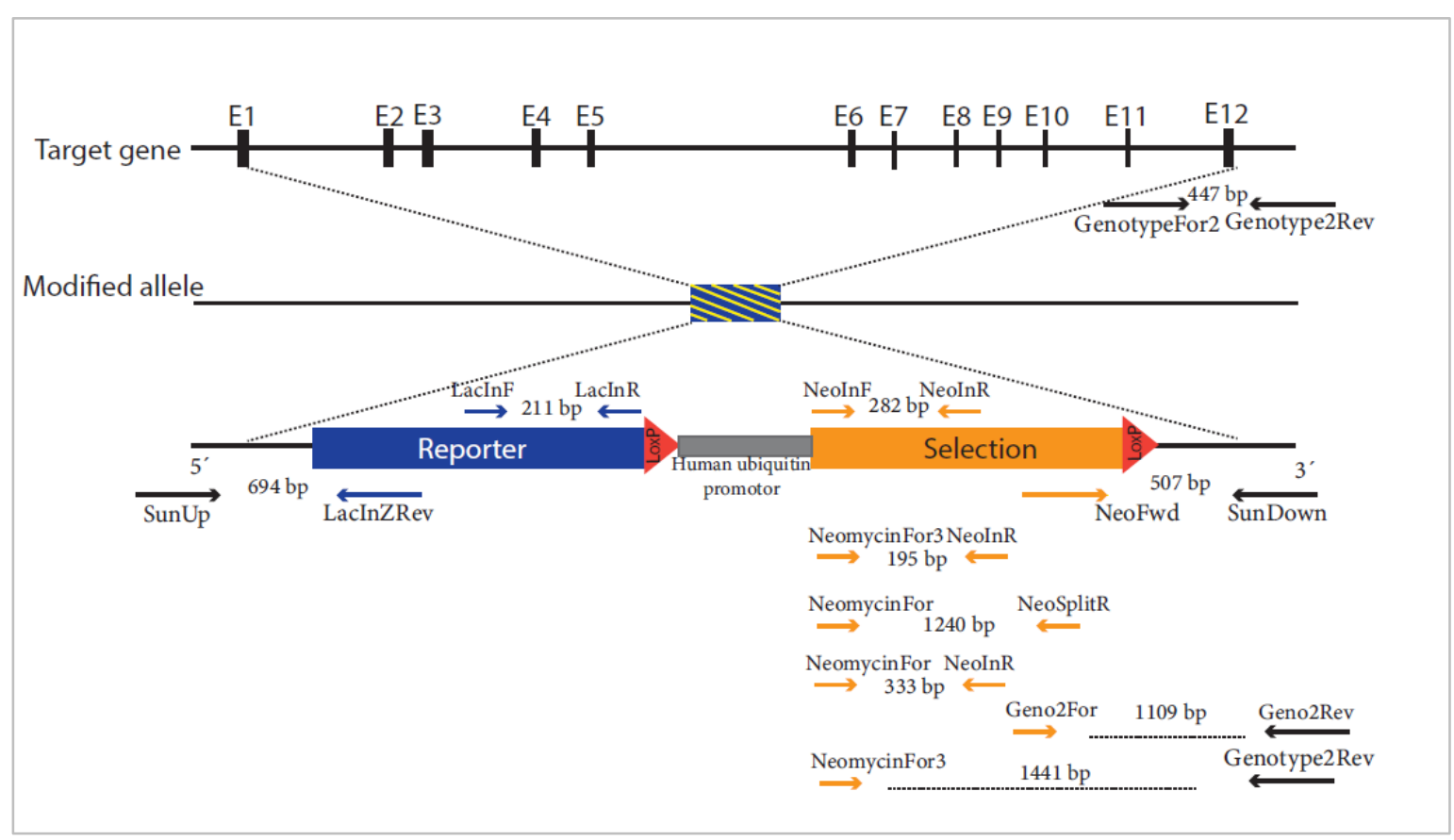

Figure 3.22: Scheme of the Sun5 knockout targeting vector. The mouse SUN5 gene consists of 12 exons. The targeted allele consists of the reporter gene LacZ (blue), followed by LoxP, human ubiquitin promoter, the selection gene neomycin (orange), and another LoxP site. This replacement cassette was integrated between the middle area of exon 1 and exon 12 of Sun5, leading to the complete depletion of Sun5 coding sequences. Positions and sizes of primers required for genotyping are illustrated. The primer pairs SunUp/LacInZRev and NeowFwd/SunDown enable the identification of the correct genomic insertion of the modified allele. The primer SunUp is situated in the upstream region of Sun5, whereas the primer LacInZRev is situated in the 5 'region of the LacZ reporter gene. On the other side, NeoFwd situates in the 3'region of neomycin cassette, whereas SunDown is situated in the downstream region of Sun5. Different pairs of primers served for genotyping and are shown as blue and orange arrows. The primer pair GenotypeFor2/ Genotype2Rev detects the wild type allele, whereas NeoFwd/SunDown, Geno2For/Geno2Rev, and NeomycinFor3/Genotype2Rev identified the recombinant allele. 
DNA from ES cells was isolated using DirectPCR Lysis Reagent-Tail as described in 2.2.5.3 and the cassette insertion was verified by PCR using the primer pair SunUp and LacZRev. The primer SunUp amplified the intronic region upstream of the Sun5 coding region. The cassette was inserted $88 \mathrm{bp}$ downstream in the exon1. The primer LacInZRev situates at the beginning of the insertion cassette LacZ (Fig. 3.22). As expected, the PCR product had a size of $\sim 602 \mathrm{bp}$ (Fig. 3.23, A). Further, the fragment obtained was isolated and the DNA was sequenced with the primer LacInZRev to verify the cassette insertion in the genome. Sequences obtained confirm the insertion of the cassette in the genome (Fig. 3.23, B).

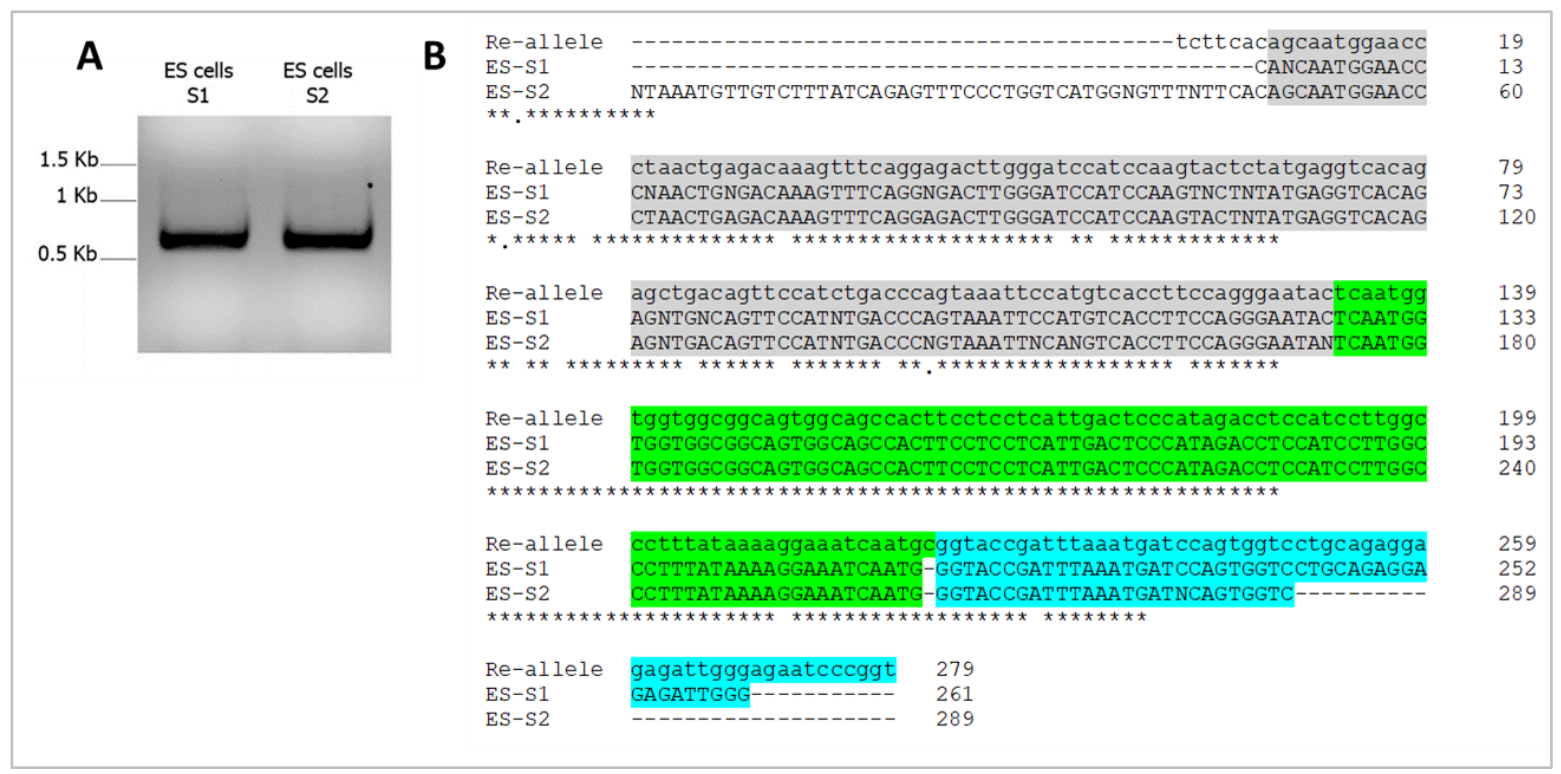

Figure 3.23 Positive genotyping of ES cells. (A) Recombinant ES cells confirm the correct LacZ-Neomycin cassette insertion in the mouse genome. The insertion was verified using the pairs of primers SunUp and LacZInRev, which amplify a fragment of $\sim 602 \mathrm{bp}$. The primer SunUp is situated in the intronic region of the wild type allele $\sim 562$ bp upstream of the LacZ region (sequence highlighted in gray and green). Primer LacZInRev is situated in the 5'region of the LacZ reporter gene and recognize a fragment of $\sim 40$ bp (light blue). ES cells-sample 1 (S1), ES cells-sample 2 (S2). (B) Multiple alignment of the recombinant allele and recombinant embryonic stem cell sequences. Recombinant allele sequence (Re-allele), ES cells -sample 1 (S1), ES cells-sample 2 (S2). The intronic sequence of the wild type allele (gray), exon1 (green), LacZ sequence (light blue).

Once the correct insertion of the cassette LacZ-Neomycin in ES cells was confirmed, the deletion of Sun5 was confirmed by qPCR before microinjection. To check the reduction of the expression of exon 7 and 9 of the Sun5 gene, the following pair of primers were used: Sun5mus-E5-For/ Sun5-mus-E5-Rev (to amplify exon7) and Sun5-mus-E8-For/ Sun5-mus-E8-Rev (to amplify exon 9) (Tab. 6.1.8). For this objective, the expression differences of the $\mathrm{Ct}$ value 
obtained from the wild-type mouse was compared with the $\mathrm{Ct}$ value from recombinant ES cells using the double delta $\mathrm{Ct}$ method. Odf1 and protamine genes were used as internal reference.

The relative $\mathrm{Ct}$ value of exons 7 and 9 was set as 1 in DNA from the wild-type mouse. The DNA from ES cells demonstrated a reduction of $\sim 50 \%$. These results demonstrate that the Sun 5 knock-out ES cells contain only one allele of Sun5 exons 7 and 9 and are therefore heterozygous for the wild-type allele (Fig. 3.24). Therefore, qPCR confirmed the proper cassette insertion and disruption of the Sun5 gene. After confirmation of the correct homologous recombination by PCR and qPCR, ES cells were used to generate chimeras.
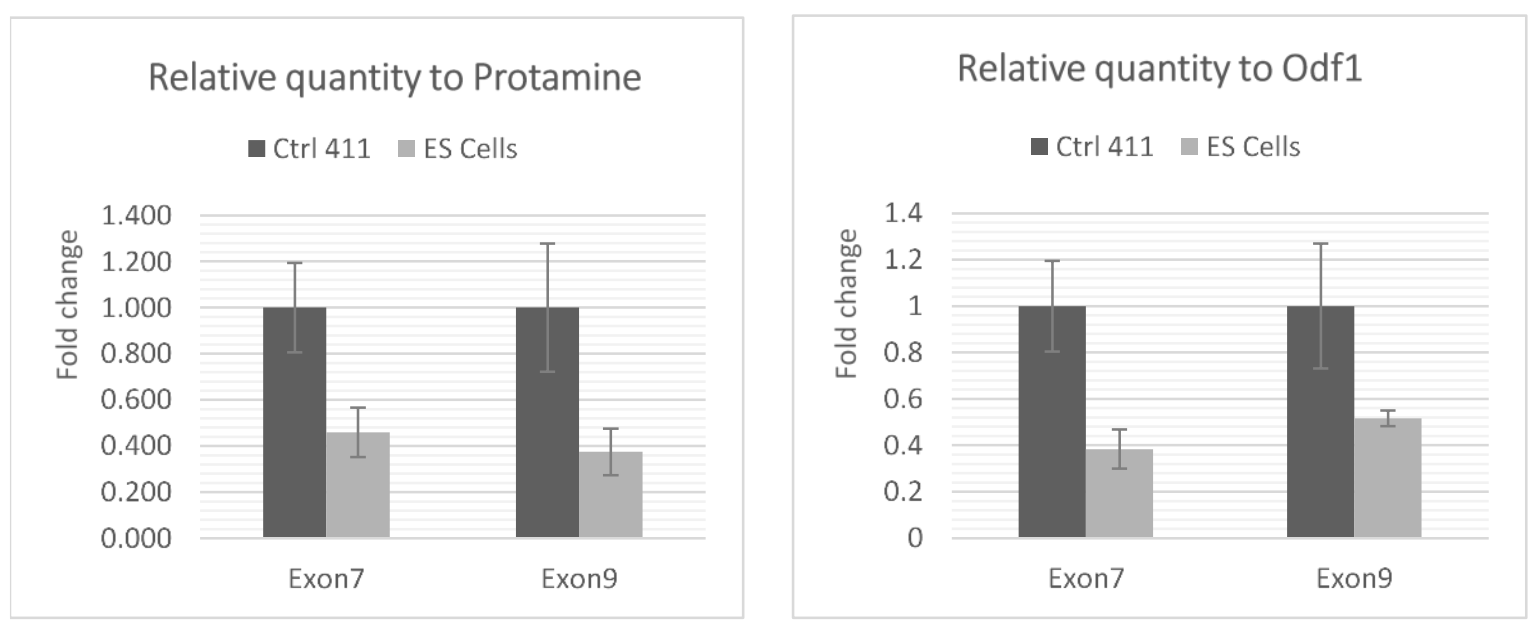

Figure 3.24. Recombinant Sun5 embryonic stem cells are heterozygous for the wild-type allele. Quantification of exons 7 and 9 of Sun5 in wild-type and recombinant ES cells, either related to the quantity of Prm1 or Odfl, respectively, always in the same probe. The relative amount of the wild-type allele (Ctrl 411 ) is 1 . In contrast, in the recombinant ES cells, the relative quantities of exons 7 and 9 are close to 0.5 when related to the quantity of either Prml or Odfl. 


\subsubsection{Genotyping of chimeras}

Genomic DNA was prepared using KAPA ${ }^{\mathrm{TM}}$ Mouse Genotyping Kit as described in 2.2.5.3. The genotyping of chimeras was performed using the primer pair NeomycinFor and NeoSplitRev (Tab. 6.1.7), which amplify a fragment of the neomycin selection gene (Fig. 3.22). An expected fragment size of $\sim 1240$ bp was amplified in 4 out of 61 chimeric mice (Fig. 3.25).

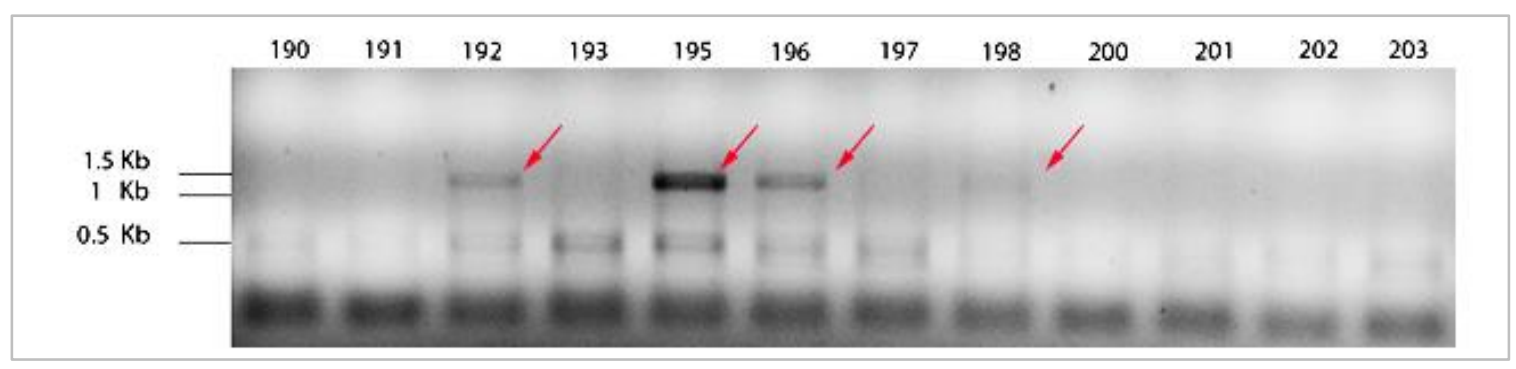

Figure 3.25. Genotyping of Sun5 chimeras. DNA obtained from chimeras was tested by PCR using the primer pair NeomycinFor and NeoSplitRev. A fragment of $~ 1240$ bp was obtained from chimeric mice \#192, $\# 195$, \#196, and \#198.

\subsubsection{Genotyping of chimeras' offspring}

Positive chimeras were crossed with $\mathrm{C} 57 \mathrm{~B} 1 / \mathrm{N}$ mice. In the first generation, 29 animals were born, which were genotyped by nested PCR. First PCR was prepared using the primer pair Geno2For and Geno2Rev (Tab.6.1.7). The primer pair amplifies part of the resistant cassette and the targeted region (Fig. 3.22). No PCR product was visibly obtained when separated by agarose gel electrophoresis (not shown). The first PCR reaction product was used as a template for the secondary PCR, and a product of $507 \mathrm{bp}$ was generated using the primers NeoFwd and Sundown (Fig. 3.26). NeoFwd is situated in the neomycin gene, whereas Sundown localizes in the 3'region flanking the cassette insertion. Two females, \#336 and \#360, were identified to carry the recombinant allele. Both females were selected for breeding with wild-type mice to establish the Spag4l/Sun5-deficient strain. 


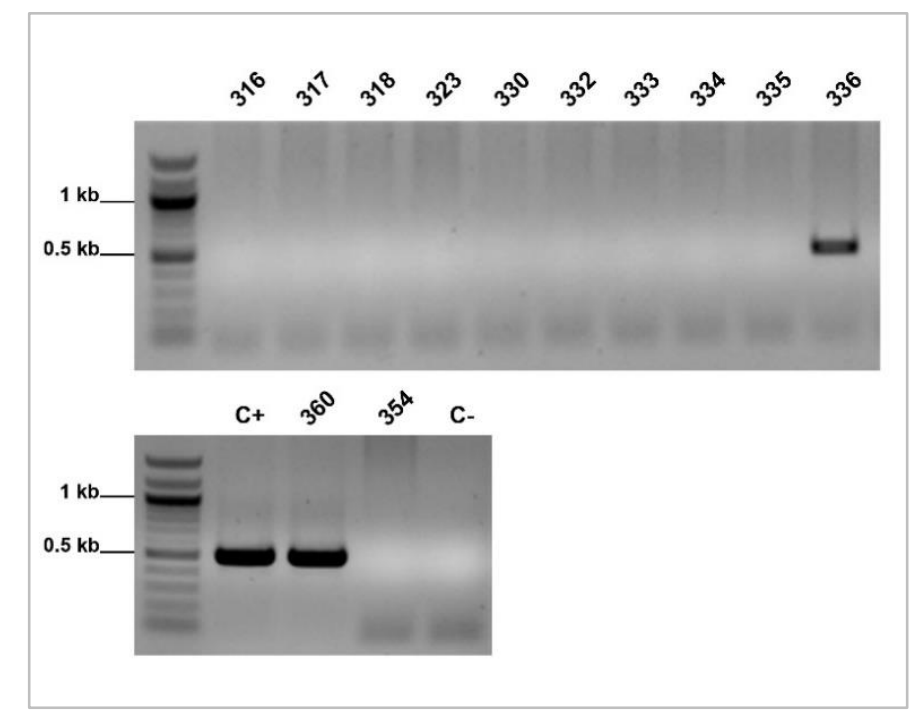

Figure 3.26. Genotyping of the first generation of Spag4l-deficient mice by nested PCR. F1 mice were genotyped by nested PCR. The first PCR reaction was performed with the primer pair Geno2For/Geno2 Rev. The second PCR was performed with primer pair NeoFwd/Sundown. The expected fragment of $507 \mathrm{bp}$ was obtained in DNA from the females \#336 and \#360. C+ corresponds to the ES cells carrying the cassette, whereas in $\mathrm{C}-$, $\mathrm{ddH}_{2} \mathrm{O}$ was used as template.

Both females were fertile and gave birth to the F2-generation. All mice of F2-generation were genotyped by nested PCR, using the primer pair NeomycinFor and NeoSplitRev for the first PCR and primers NemoycinFor3 and NeoInRev for the secondary PCR. A product of $\sim 195 \mathrm{bp}$ was obtained as expected in some samples. To confirm the correct transmission of the insertion cassette, some PCR products were sequenced, confirming the presence of the neomycin gene (Fig. 3.27).

Heterozygous animals, that tested positive for the presence of the insertion cassette and wildtype allele by genotyping, were intercrossed to generate homozygous Spag4-deficient mice. The breeding scheme and the progenies genotyped positive for the presence of the insertion cassette is shown in figure 3.28. Genotyping was performed using DNA isolated by three different methods. For the PCR reactions, different combinations of primers were used, which are described in figure 3.22 . 


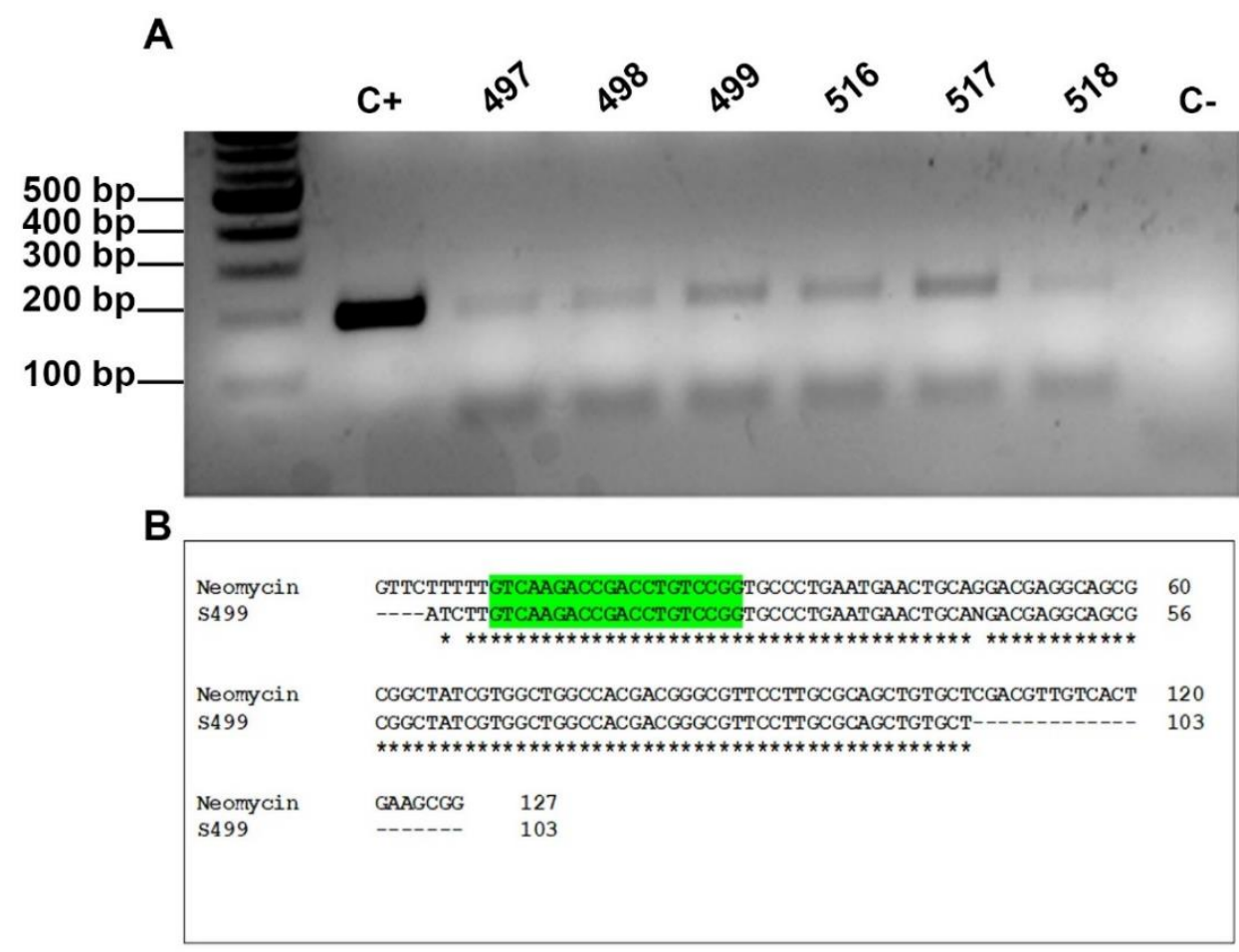

Figure 3.27. Genotyping of the second generation of Spag4l-deficient mice (A) Genotyping was performed by nested PCR using the primer pair NeomycinFor/NeoSplitRev for the first PCR reaction, and the primer pair NemoycinFor3/NeoInRev for the secondary PCR reaction. The expected fragment of 195 bp was amplified. The PCR product was sequenced (B) Alignment of DNA sequence from sample \#499 with the neomycin sequence revealed amplification of the neomycin gene. The primer sequence of NemoycinFor3 is shown in green.

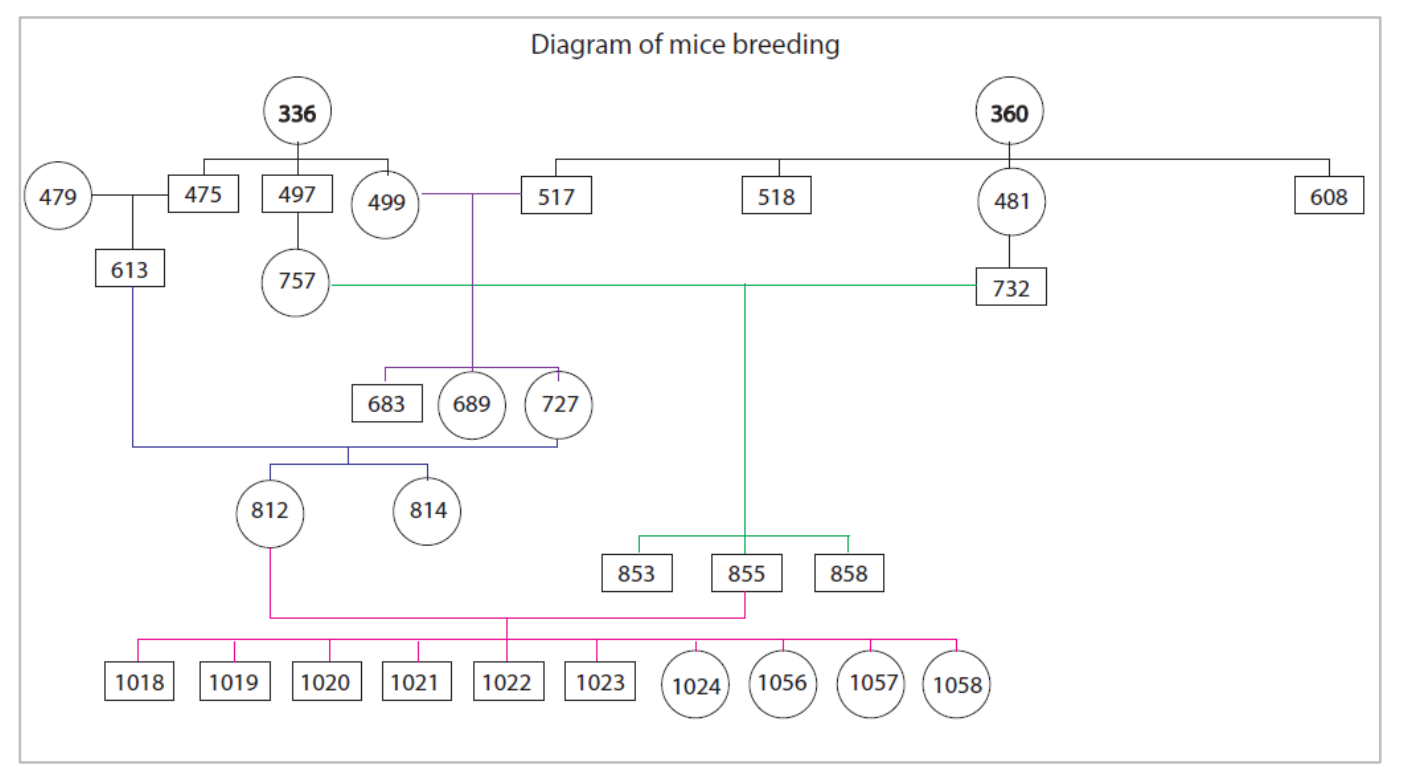

Figure 3.28. Breeding scheme to generate homozygous-deficient $\mathrm{Spag}^{-{ }^{-/}}$mice. Heterozygous animals were intercrossed to generate homozygous Spag4-deficient mice. Five generations of mice are represented in the current scheme. 
Additionally, to confirm heterozygosity, samples were analyzed by qPCR to check whether the quantity of exons 7 and 9 of Spag $4 l$ are reduced. qPCR was performed using the same conditions as described for ES cells (2.2.5.5). The primer pairs Sun5-mus-E5-For/ Sun5-mus-E5-Rev and Sun5-mus-E8-For/ Sun5-mus-E8-Rev were used to amplify exon 7 and 9, respectively. DNA from a wild-type mouse was used as reference and protamine was used as an internal control gene. Relative quantity was calculated using the $2^{-\Delta \Delta C t}$ method. As shown in Fig. 3.29, a notable reduction of the exon 7 and 9 was found in some samples. Therefore, those animals that were positive genotyped for heterozygosity by PCR and qPCR were selected for breeding (Fig. 3.28).

After four generations of mice breeding, several animals were positive for the selection gene neomycin. However, all mice obtained were heterozygous since the wild-type allele was detected in all animals by using the primer pair GenotypeFor2 and GenotypeRev2 (Fig. 3.30). Thus, besides intercrossing of heterozygous animals, no homozygous Spag4-deficient mice could be obtained. After reevaluation of genotyping results, we concluded that transmission failed. The project was therefore stopped.

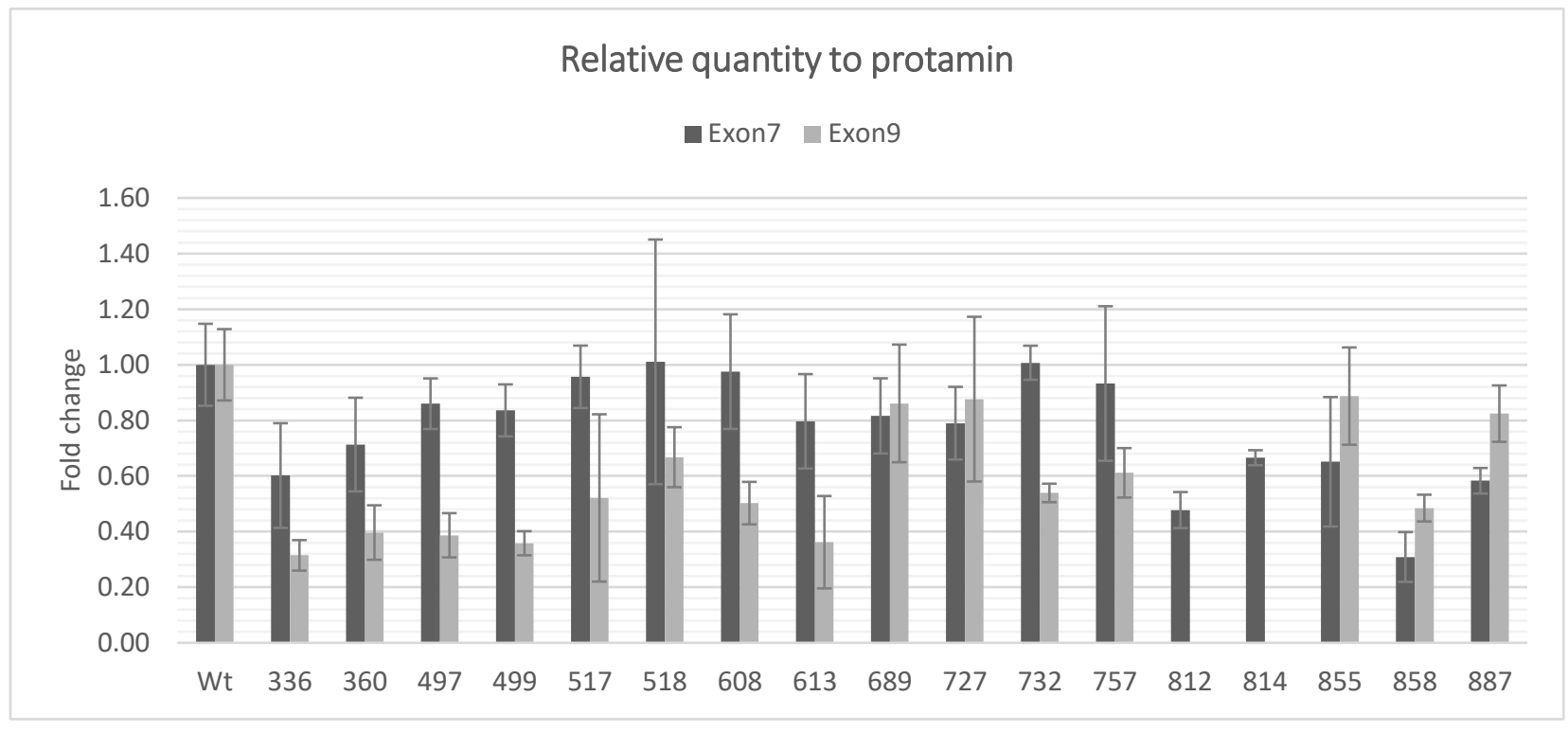

Figure 3.29. Relative quantity of exon 7 and 9 of the offspring from heterozygous mice. The quantity of exons 7 and 9 relative to protamine was calculated using DNA from a wild type as reference. Exon 9 shows a reduction in most of the animals analyzed $(336,360,497,499,517,518,608,613,732,757$ and 858), whereas exon 7 is reduced in a few samples $336,812,855,858,887$. Relative gene quantity was calculated using the $2^{-\Delta \Delta C t}$ method. 


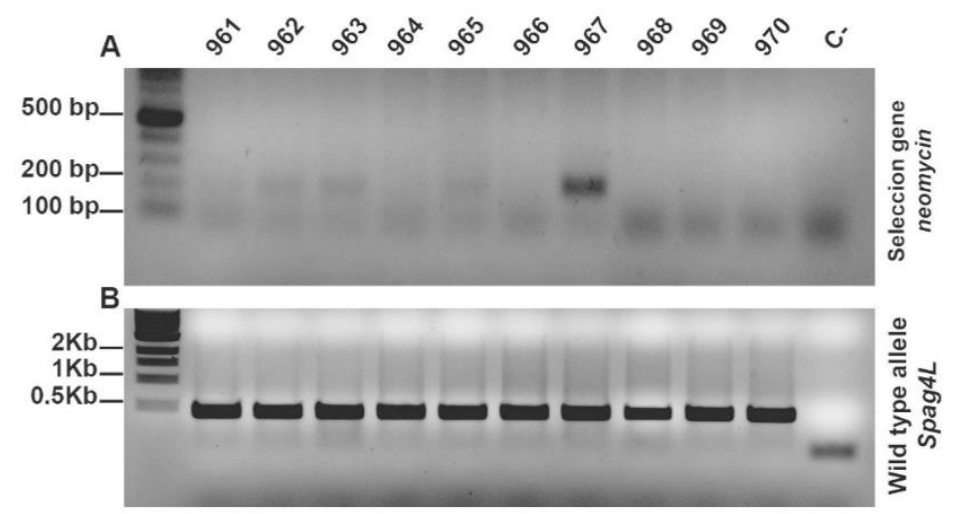

Figure 3.30. Genotyping example of the fourth generation. Offspring generated after mating of heterozygous mice \#732 and \#812. (A) Primers NeomycinFor3 and NeoInRev amplified the neomycin fragment $\sim 195$ bp, confirming Spag $4 l$ deficiency. (B) Primers GenotypeFor2 and GenotypeRev2 amplified a fragment of $447 \mathrm{bp}$ of the wild type allele and was used to detect the wild-type allele. Both pairs of primers amplified a fragment in sample \#967, thus confirming the heterozygosity of this mouse. 


\subsection{Identification of proteins that collaborate in the formation of the HTCA}

\subsubsection{CCDC42 localizes to manchette, HTCA and tail and interacts with ODF1 and ODF2 in the formation of the male germ cell cytoskeleton}

This chapter describes the identification of CCDC42 as an interacting partner of ODF1 and ODF2, suggesting its collaboration in the HTCA formation. The coiled-coil domain containing 42 (CCDC42) is an essential protein involved in the formation of the HTCA and sperm flagella. In early elongating spermatids, CCDC42 localizes to the manchette and perinuclear ring. In the later stages of spermiogenesis, CCDC42 locates in the connecting piece and to the sperm tail. Furthermore, CCDC42 interacts physically with ODF1, and both proteins exhibit a similar distribution in the sperm tail.

Moreover, CCDC42 interacts with the outer dense fiber protein 2 (ODF2). ODF2 is a known component of the sperm tail and centrosome/basal body in somatic cells. Similarly, CCDC42 has been identified as a centrosomal component in somatic cells, albeit previous reports restricted $C c d c 42$ expression to testis and brain. However, these results indicate that another Ccdc42 isoform is expressed in somatic cells. Hence, CCDC42 interacts with the cytoskeletal proteins ODF1 and ODF2, suggesting its potential role as a scaffold protein in the formation of the connecting piece and sperm tail.

Constanza Tapia Contreras and Sigrid Hoyer-Fender

Author Contributions: CTC did the experiments and prepared the figures. SH-F was the project leader and wrote the manuscript.

Status of manuscript: Published in Frontiers in Cell and Developmental Biology in 2019. 


\section{OPEN ACCESS}

Edited by: Tomer Avidor-Reiss, The University of Toledo, United States

Reviewed by:

Maria Eugenia Teves, Virginia Commonwealth University, United States Bénédicte Durand, Université Claude Bernard Lyon 1 , France

*Correspondence: Sigrid Hoyer-Fender shoyer@gwdg.de

Specialty section: This article was submitted to Cell Growth and Division, a section of the journal Frontiers in Cell and Developmental Biology

Received: 31 May 2019 Accepted: 18 July 2019 Published: 14 August 2019

Citation:

Tapia Contreras $C$ and Hoyer-Fender S (2019) CCDC42 Localizes to Manchette, HTCA and Tail and Interacts With ODF1 and ODF2 in the Formation of the

Male Germ Cell Cytoskeleton.

Front. Cell Dev. Biol. 7:151. doi: 10.3389/fcell.2019.00151

\section{CCDC42 Localizes to Manchette, HTCA and Tail and Interacts With ODF1 and ODF2 in the Formation of the Male Germ Cell Cytoskeleton}

\author{
Constanza Tapia Contreras and Sigrid Hoyer-Fender* \\ Johann-Friedrich-Blumenbach-Institute of Zoology and Anthropology - Developmental Biology, Göttingen Center \\ for Molecular Biosciences (GZMB), Georg-August-University of Göttingen, Göttingen, Germany
}

Terminal differentiation of male germ cells into functional spermatozoa requires shaping and condensation of the nucleus as well as the formation of sperm-specific structures. A transient microtubular structure, the manchette, is mandatory for sperm head shaping and the development of the connecting piece and the sperm tail. The connecting piece or head-to-tail coupling apparatus (HTCA) mediates the tight linkage of sperm head and tail causing decapitation and infertility when faulty. Using mice as the experimental model, several proteins have already been identified affecting the linkage complex, manchette or tail formation when missing. However, our current knowledge is far too rudimentary to even draft an interacting protein network. Depletion of the major outer dense fiber protein 1 (ODF1) mainly caused decapitation and male infertility but validated binding partners collaborating in the formation of sperm-specific structures are largely unknown. Amongst all candidate proteins affecting the HTCA when missing, the structural protein CCDC42 attracted our attention. The coiled-coil domain containing 42 (CCDC42) is important for HTCA and sperm tail formation but is otherwise largely uncharacterized. We show here that CCDC42 is expressed in spermatids and localizes to the manchette, the connecting piece and the tail. Beyond that, we show that CCDC42 is not restricted to male germ cells but is also expressed in somatic cells in which it localizes to the centrosome. Although centrosomal and sperm tail location seems to be irrespective of ODF1 we asked whether both proteins may form an interacting network in the male germ cell. We additionally considered ODF2, a prevalent protein involved in the formation of spermatid-specific cytoskeletal structures, as a putative binding partner. Our data depict for the first time the subcellular location of CCDC42 in spermatids and deepen our knowledge about the composition of the spermatid/sperm-specific structures. The presence of CCDC42 in the centrosome of somatic cells together with the obvious restricted male-specific phenotype when missing strongly argues for a compensatory function by other still unknown proteins most likely of the same family.

Keywords: spermiogenesis, HTCA, tail, CCDC42, centrosome, ODF2, ODF1 


\section{INTRODUCTION}

The transformation of spermatids into terminally differentiated sperm is a key event in spermatogenesis. Meiosis II generates spermatids of spherical shape that are then gradually transformed by shaping and condensation of the nucleus and the formation of acrosome and sperm tail, finally resulting in the mature spermatozoon (Fawcett, 1975; Russell et al., 1990; Jan et al. 2012). There is circumstantial evidence indicating that the shaping of the nucleus and the assembly of the sperm tail is provoked by a transient microtubular structure, the manchette (Clermont et al., 1993; Kierszenbaum, 2002; Kierszenbaum et al., 2011). The manchette forms during the acrosomal phase of spermiogenesis and disassembles during the maturation phase (Clermont et al., 1993). In mice, it is first seen in step 8 spermatids and disassembles prior to the formation of the sperm mid-piece around steps 13-14 (O'Donnell and O'Bryan, 2014; Lehti and Sironen, 2016). The manchette consists of a perinuclear mantle of microtubules emanating from the perinuclear ring (Fawcett et al., 1971; Rattner and Brinkley, 1972; Dooher and Bennett, 1973; Rattner and Olson, 1973; Wolosewick and Bryan, 1977; Clermont et al., 1993). However, detection of plus-end tracking proteins as EB3 and CLIP-170 at the perinuclear ring together with the absence of the minus-end binding protein $\gamma$-tubulin strongly argues against the perinuclear ring as the microtubule nucleation site (Akhmanova et al., 2005; Kierszenbaum et al., 2011; Lehti and Sironen, 2016). Instead, supporting evidence indicates that the centriolar adjunct serves as a nucleator of manchette microtubules with their plus ends reaching toward the perinuclear ring (Fawcett and Phillips, 1969; Lehti and Sironen, 2016; Fishman et al., 2018). The manchette is linked to the nuclear membrane and this is essential for nuclear shaping. The presence of rod-like elements that link the manchette to the nuclear envelope has first been demonstrated by electron microscopy studies (Russell et al., 1991). Supportively, deletion of SUN4, a testis-specific nuclear membrane protein and component of the linker of nucleoskeleton and cytoskeleton complex (LINC) caused detachment of the manchette and consequently roundheaded sperm (Calvi et al., 2015; Pasch et al., 2015; Yang et al., 2018a). The importance of the manchette for nuclear shaping and male fertility is furthermore exemplified by the seminal discovery of the genetic cause underlying the $a z h$ phenotype in mice. Male azh mice are infertile due to a malformed manchette, abnormal spermatozoon head morphology, tail abnormalities and decapitation all caused by a deletion in the Hook1 gene (Mendoza-Lujambio et al., 2002). (Review in: Chen et al., 2016). However, Hook1 is a microtubule-binding protein and most likely responsible for the cross-linking of the manchette microtubules, whereas SUN4 is expected to be an inner nuclear membrane protein. Thus, the true nature of the rod-like elements that link the manchette to the nucleus is still unknown.

The observation of sperm decapitation indicated that the manchette is involved in sperm head to tail coupling and/or development of the sperm tail. Consequently, it was suggested that molecules required for the developing basal body/connecting piece and the sperm tail were delivered via intra-manchette transport meaning that the manchette functions as a track in supporting the delivery of molecules (Kierszenbaum, 2001, 2002; Kierszenbaum et al., 2011). Contradictory, however, are the observations that the manchette is assembled when the axoneme is already developed and that the sperm tail develops irrespective of the detachment of the manchette in SUN4-deficient spermatids (Lehti and Sironen, 2017; Yang et al., 2018a).

The sperm tail develops from the basal body that itself is a derivative of the former centrosome. In spermatids, the daughter centriole of the centrosome is transformed into the proximal centriole, which acts as a seed for the formation of the connecting piece, and inserts into the nuclear indentation (Fawcett and Phillips, 1969). The perpendicular positioned mother centriole is transformed into the distal centriole, which acts as the basal body to initiate sperm tail development. Later on, the distal centriole disintegrates leaving the centriolar vault. The axoneme, the microtubule-based core structure, is the prolongation of the distal centriole that is surrounded by accessory structures as the nine prominent outer dense fibers (ODFs) and the fibrous sheath (FS) in the sperm tail. The ODFs are descending from the segmented columns formed at the proximal centriole of the head-to-tail coupling apparatus (HTCA). They accompany the microtubule doublets of the axoneme throughout the length of the tail whereas the FS is present only in the principal piece. The accessory fibers are important for stiffening the sperm tail thus supporting the elastic recoil of the sperm tail and protecting against shearing forces (Baltz et al., 1990; Lindemann, 1996). At the proximal region of the sperm tail, at the mid-piece, the mitochondrial sheath surrounds axoneme and ODFs.

The HTCA or connecting piece develops from the centrosome. It is an articular structure at the neck region mediating the tight connection between the sperm tail and the nucleus. Although the protein composition of the HTCA is far from being known, a couple of proteins have already been identified that are essential for the formation of the HTCA and/or the sperm tail. One protein essential for the tight connection of sperm head and tail is the outer dense fiber protein 1 (ODF1; also named HSPB10) (Burfeind and Hoyer-Fender, 1991; Schalles et al., 1998; Fontaine et al., 2003). Depletion of ODF1 caused sperm decapitation and male infertility in mice (Yang et al., 2012, 2014). A few interacting proteins have been identified, e.g., the outer dense fiber protein 2 (ODF2), which is a major protein of the sperm tail accessory fibers (Shao et al., 1997). Beyond that, validated ODF1 interacting proteins that are supposed to collaborate in the formation of sperm-specific structures are currently unknown. Proteins known to affect the HTCA or the sperm tail when missing are ideal candidates as putative interaction partners. We, therefore, focused on structural proteins with a reported effect on HTCA and sperm tail formation as putative interaction partners of ODF1. We asked here, whether the coiled-coil domain containing 42 (CCDC42) protein acts as a node in the ODF1 network. Ccdc42 is specifically expressed in testis and brain and its deletion causes male sterility in mice with malformation of the HTCA and the sperm tail. Beyond that, no further phenotypes are evident (Pasek et al., 2016). CCDC42 (coiled-coil domain containing 42) belongs to the CFAP73 family and is a paralog of CFAP73. It contains the DUF4200, the domain of unknown function 
that is shared by a couple of coiled-coil domain proteins and cilia-and flagella-associated proteins as CFAP73. The phenotype of $C c d c 42$-deficient mice suggested a male germ cell-specific function, but its expression and sub-cellular location is so far unknown. We explored here putative interacting proteins of CCDC42 in male germ cells and analyzed its expression and sub-cellular location. We show that CCDC42 is recruited to the manchette and the sperm tail and is specifically enriched in the perinuclear ring of the manchette and the HTCA. Pull down and co-IP experiments both indicated binding to ODF1 and ODF2. Furthermore, CCDC42 localizes to the centrosome/basal body not only in male germ cells but also in somatic cells. Revision of $C c d c 42$ expression by RT-PCR demonstrated wide-spread expression in somatic tissues. The co-localization of CCDC42 with microtubule-based structures as the manchette and the centrosome/HTCA suggests that CCDC42 is involved in their formation by generating a rigid scaffold. However, as no further phenotypes are evident when $C c d c 42$ is missing its function in somatic cells most likely might be taken over by other members of the family.

\section{MATERIALS AND METHODS}

\section{Ethics Statement}

All mouse experiments were reviewed and approved by the local ethic commission. License for animal experiments has been obtained by the Institute of Human Genetics and the MaxPlanck-Institute for Experimental Medicine, Göttingen. The guidelines of the German Animal Welfare Act (German Ministry of Agriculture, Health and Economic Cooperation) were strictly followed in all aspects of mouse work.

\section{cDNA Synthesis and RT-PCR}

Total RNA was prepared form adult mouse tissues as well as from NIH3T3 mouse fibroblasts using peqGOLD RNApure $^{\mathrm{TM}}$ (PeqLab, Erlangen, Germany) following the recommendations of the manufacturer. Total RNA was digested with Ambion ${ }^{\circledast}$ TURBO DNA-free ${ }^{\mathrm{TM}}$ DNase (Life Technologies) followed by cDNA synthesis using Maxima First Strand cDNA Synthesis (Thermo Fisher Scientific). RTPCR for detection of transcribed sequences was performed using the following primer combinations: Ccdc42-Nterm_For (GTGGCACTGTCACTCACC) and C-terminal-Ccdc42-Rev (GGCTCACCAGGAACCTTCTC) generating the full-length product of $1093 \mathrm{bp}$, Ccdc42-For2 (GGAGACCGAGAATCCA GCC) and Ccdc42-Rev2 (CCGTTGGAATGCCTCCTTCT) for amplification of $305 \mathrm{bp}$ of the $5^{\prime}$ region (exons $1+2$ ), C-terminal-Ccdc42-For (GGAATCCACCCAAGTGTCCC) and C-terminal-Ccdc42-Rev (GGCTCACCAGGAACCTTCTC) generating a fragment of $203 \mathrm{bp}$ of the conserved $3^{\prime}$ region (exons $6+7$ ), Ccdc42-Exon5-For (GAAGAGATCCACGAGGTG) and C-terminal-Ccdc42-Rev for amplification of exons 5-7 (expected fragment size $535 \mathrm{bp}$ ), Gapdh-For (GTATGA CTCCACTCACGGCA) and Gapdh-Rev (GTCAGATCCACGA CGGACAC) generating a fragment of $594 \mathrm{bp}$.

\section{Plasmid Constructs}

PCR amplification based on the Ensembl reference sequence NM_177779 by using the following primers: Ccdc42-NheI-For (5'-GGCTGTTAGGTAGCTAGCGCAAC CATGAGTTTGGG-3 $\left.{ }^{\prime}\right)$ and Ccdc42-HindIII-Rev (5'-GTTA CTTCCTTAAGCTTGCCATCCGGACTTGCTGTbTG- ${ }^{\prime}$ ), each primer containing restriction enzyme recognition sites. The full-length coding sequence of the $C c d c 42$ isoform 203 was first cloned into pJET1.2/blunt (Thermo Fisher Scientific) followed by NheI/HindIII digestion and sub-cloning into pCR3.1-Cherry resulting in an in-frame fusion with the C-terminal Cherrytag (pCR3.1, Invitrogen). The full-length coding region of Odf1 was N-terminally fused to ECFP in PECFP-C1 (Clontech Lab.). Odf2 was C-terminally fused to EGFP in pEGFP-N1 (Clontech Lab., \#U55762) generating the full length construct 13.8NC-EGFP (Donkor et al., 2004). Sequencing always revealed correct reading frames.

\section{Cell Culture and Immunocytochemistry}

NIH3T3 (ATCC CRL-1658) or HEK-293 cells (ATCC CRL1573) were maintained in Dulbecco's Modified Eagle's Medium (DMEM) supplemented with $10 \%(\mathrm{v} / \mathrm{v})$ fetal bovine serum (FBS), $1000 \mathrm{U} / \mathrm{ml}$ penicillin, $1000 \mu \mathrm{g} / \mathrm{ml}$ streptomycin, and $20 \mathrm{mM}$ L-Glutamine (all Gibco) at $37^{\circ} \mathrm{C}$ and $5 \% \mathrm{CO}_{2}$. NIH3T3 cells were grown on coverslips in 6-well plates and transfected using EndoFectin ${ }^{\mathrm{TM}}$ Max Transfection Reagent, following the recommendations of the manufacturer (GeneCopoeia). Twentyfour hours after transfection, cells were washed in phosphatebuffered saline (PBS) and fixed in methanol for $10 \mathrm{~min}$ at $-20^{\circ} \mathrm{C}$. Specimens were then permeabilized in $0.3 \%$ TritonX-100 in PBS for $10 \mathrm{~min}$ at room temperature and blocked for $1 \mathrm{~h}$ using blocking solution (PBS containing $1 \%$ BSA and $0.3 \%$ TritonX$100)$. Samples were incubated with primary antibodies toward CCDC42 (ARP52735_P050, antibodies-online ABIN2785068), Pericentrin (PRB432C, Covance), acetylated tubulin (6-11B1; Sigma-Aldrich), gamma-tubulin (GTU-88, Sigma-Aldrich), ODF1 (ABIN4341345, antibodies-online), and GFP (raised in rabbit, self-made) at $37^{\circ} \mathrm{C}$ for $1 \mathrm{~h}$. Secondary antibodies used are goat anti-mouse-IgG DyLight 488 (\#35503, Thermo Fisher Scientific), and goat anti-rabbit MFP590 (\#MFP-A1037, Mobitec). DNA was counterstained with DAPI. Images were taken by confocal microscopy (LSM 780, Zeiss) and processed using Adobe Photoshop 7.0.

\section{Immunocytology on Testicular Cell Suspensions}

Fresh testes from laboratory mice of strain C57/B16, or frozen epididymides from wild-type mice or Odf1-ko mice (Yang et al., 2012) were minced in PBS, transferred onto superfrost slides, and fixed either in $2 \%$ or $3.7 \%$ paraformaldehyde in PBS for $20 \mathrm{~min}$. Cells were permeabilized afterward in $0.3 \%$ Triton $\mathrm{X}-100$ in PBS for $10 \mathrm{~min}$, followed by blocking for $1 \mathrm{hr}$ in blocking solution (PBS containing $1 \%$ BSA and $0.3 \%$ Triton X100). The following antibodies were used for immunocytology: anti- $\alpha$-tubulin (mouse monoclonal, DM1A, Calbiochem), antiacetylated tubulin (6-11B-1, Sigma-Aldrich), anti-CCDC42 


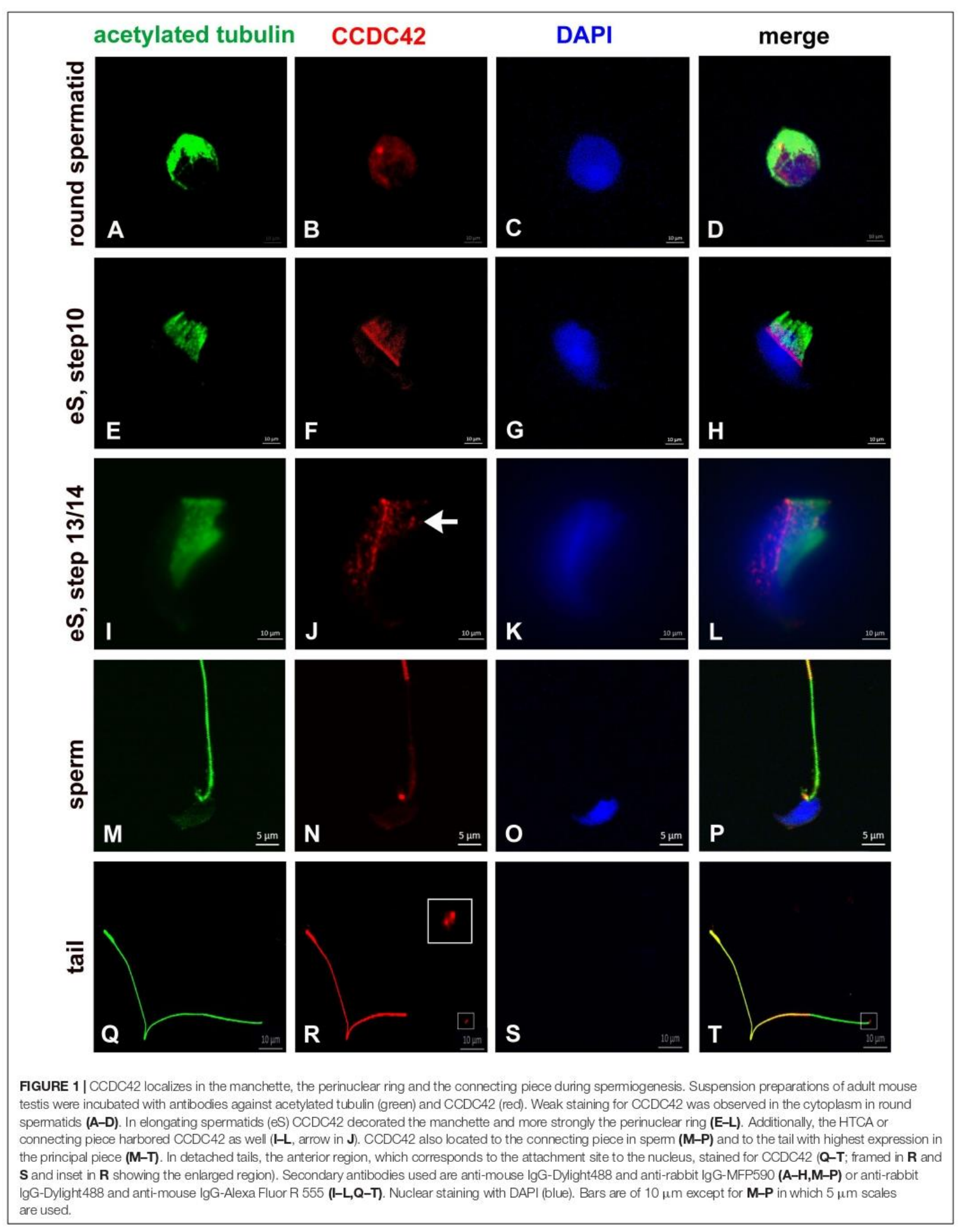



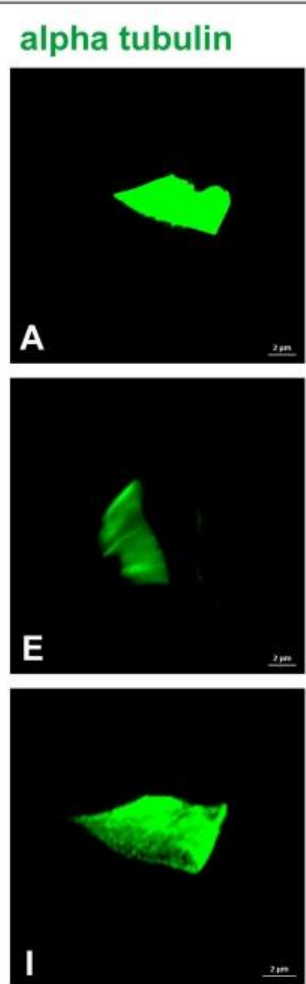
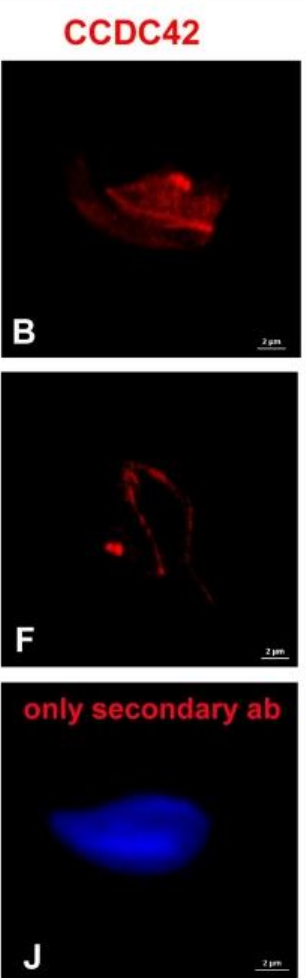

\section{DAPI}
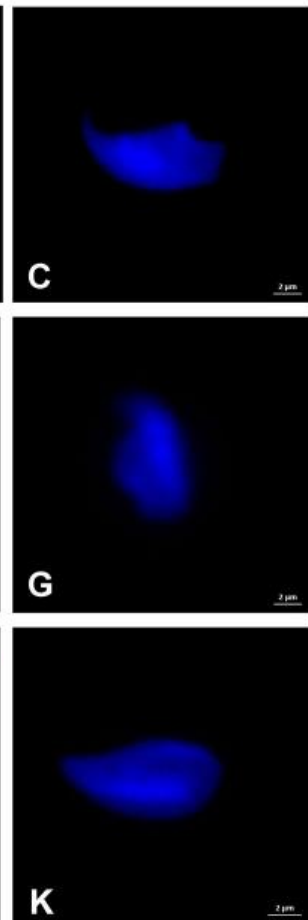

merge
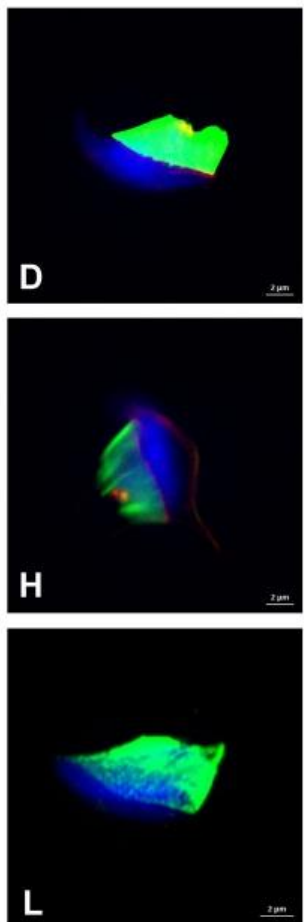

FIGURE 2 |CCDC42 locates to manchette, perinuclear ring and connecting piece. Suspension preparations of adult mouse testis were incubated with antibodies against $\alpha$-tubulin (A,E,I; green) and CCDC42 (B,F; red) and subsequently detected with the secondary antibodies anti-mouse lgG Dylight488 and anti-rabbit IgG MFP590 (A-D,I-L) or anti-mouse IgG-Dylight488 and anti-rabbit lgG-Alexa Fuor R555 (E-H). In the control, the antibody against CCDC42 was omitted (I-L). Nuclear counterstain with DAPI (blue). Bars: $2 \mu \mathrm{m}$

(ARP52735_P050; antibodies-online ABIN2785068), anti-ODF1 (antibodies-online, ABIN4341345), guinea pig anti-SUN4 (selfmade, Manfred Alsheimer, Würzburg). Primary antibodies were detected using different combinations of secondary antibodies as goat anti-mouse-IgG Alexa Fluor $555 \operatorname{IgG}(\mathrm{H}+\mathrm{L})$ (A21422, Molecular Probes) and goat anti-rabbit-IgG DyLight 488 (\#35553, Thermo Fisher Scientific), goat anti-mouse-IgG DyLight 488 (\#35503, Thermo Fisher Scientific) and goat anti-rabbit-MFP590 (\#MFP-A1037, Mobitec), goat anti-mouseIgG DyLight 488 (\#35503, Thermo Fisher Scientific) and goat anti-rabbit-IgG $(\mathrm{H}+\mathrm{L})$ Alexa Fluor R 555 (F[ab]2 fragment; \#A21430, Life Technologies), and goat anti-guinea pig-IgG Cy3 (Dianova \#106-166-003). DNA was counterstained with DAPI (4', 6-Diamidino-2-phenylindole; Sigma D-9542), and the acrosome was decorated with FITC-labeled peanut lectin (PL-FITC). Images were taken by confocal microscopy (LSM 510, Zeiss) and processed using Adobe Photoshop 7.0. In some pictures, the fluorescent colors are replaced by pseudo-colors.

\section{Co-immunoprecipitation}

HEK-293 cells were transfected using EndoFectin ${ }^{\mathrm{TM}}$ Max Transfection Reagent (GeneCopoeia), and $24 \mathrm{~h}$ post-transfection harvested by trypsinization followed by two rinses in PBS. The cell pellet was resuspended in $1 \mathrm{ml}$ lysis buffer $(150 \mathrm{mM}$ $\mathrm{NaCl}, 1 \%$ Nonidet P40, $0.5 \%$ sodium deoxycholate, $0.1 \%$ SDS, $50 \mathrm{mM}$ Tris- $\mathrm{HCl}, \mathrm{pH}$ 7.6, containing protease inhibitor cocktail (Halt Protease Inhibitor Cocktail 100x, Thermo Fisher Scientific, \#78438). Subsequently, cell lysates were passed 10x through a syringe with a 21 -gauge needle, followed by 3 -times sonication for $45 \mathrm{~s}$ each. The soluble fraction was obtained by centrifugation at $15,000 \times g$ for $15 \mathrm{~min}$ at $4^{\circ} \mathrm{C}$. The supernatant was split into three parts and incubated with different antibodies for co-IP, either with rabbit anti-GFP (self-made), rat anti-RedFP (5F8, Chromotek), or rabbit anti-GAL4 (DBD) (sc-577, Santa Cruz Biotechnology). Protein G agarose beads (Thermo Fisher Scientific) were washed three times with lysis buffer and the antibody/supernatant mixture was then added. Samples were incubated overnight at $4^{\circ} \mathrm{C}$ on a rotating wheel. Afterward, beads were washed four times with lysis buffer, and bound proteins obtained by suspension of beads in SDS sample buffer and heating at $95^{\circ} \mathrm{C}$ for $10 \mathrm{~min}$. Proteins were fractionated on SDSPAGE and transferred onto nitrocellulose membrane (Amersham Hybond-ECL, GE Healthcare) (Laemmli, 1970; Towbin et al., 1979). The membrane was incubated in blocking solution $[5 \%$ dry milk in TBST (10 mM Tris- $\mathrm{HCl}, \mathrm{pH} 7.6,150 \mathrm{mM} \mathrm{NaCl} 0.05 \%$ 


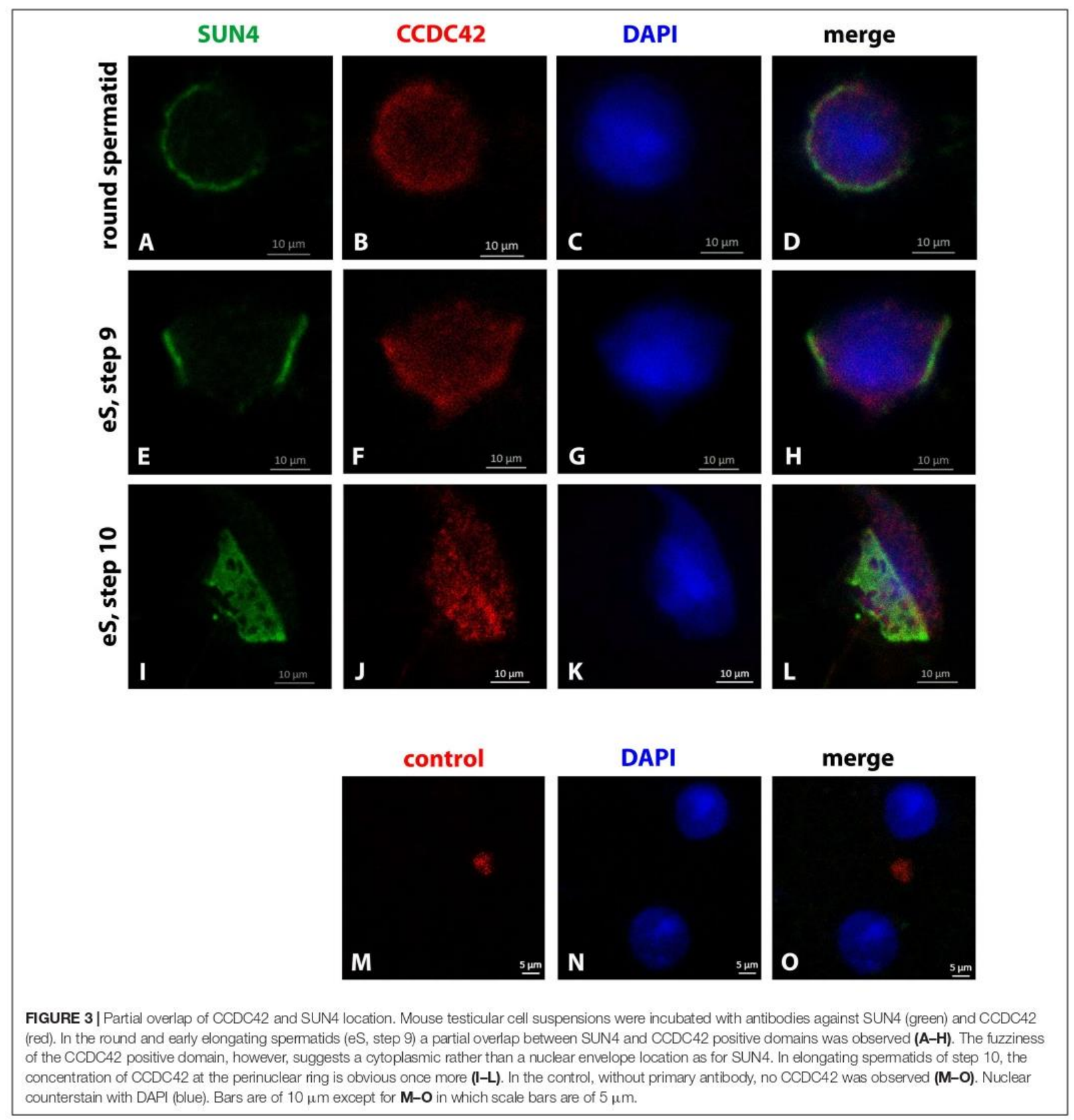

Tween 20)] for $1 \mathrm{~h}$. Membranes were incubated overnight at $4^{\circ} \mathrm{C}$ with primary antibodies either rabbit anti-GFP (self-made), mouse anti-GFP (MAB3580, Chemicon), or rat anti-RedFP (5F8, Chromotek) in blocking solution. Following washing of membranes in TBST, they were incubated with horseradish peroxidase-conjugated secondary antibodies either anti-rabbit IgG, anti-mouse IgG, or anti-rat IgG (Jackson ImmunoResearch, WestGrove, PA, United States or Sigma Biosciences, St Louis), respectively. Chemiluminescence detection was performed using
ClarityMax Western ECL Substrate (Bio-Rad, \#1705062) and images captured with Chemdoc (Bio-Rad).

\section{Pull Down Assay}

Preparation of bacterially expressed and refolded His-tagged ODF2-fusion protein (6xHis-13.8NC) was essentially as described in Yang et al. (2018b). Ni-NTA agarose (Qiagen $\mathrm{GmbH}$, Hilden) was washed in wash buffer $(50 \mathrm{mM} \mathrm{NaPi}$, $500 \mathrm{mM} \mathrm{NaCl}, 30 \mathrm{mM}$ imidazole, $\mathrm{pH} 7.6$, containing protease 


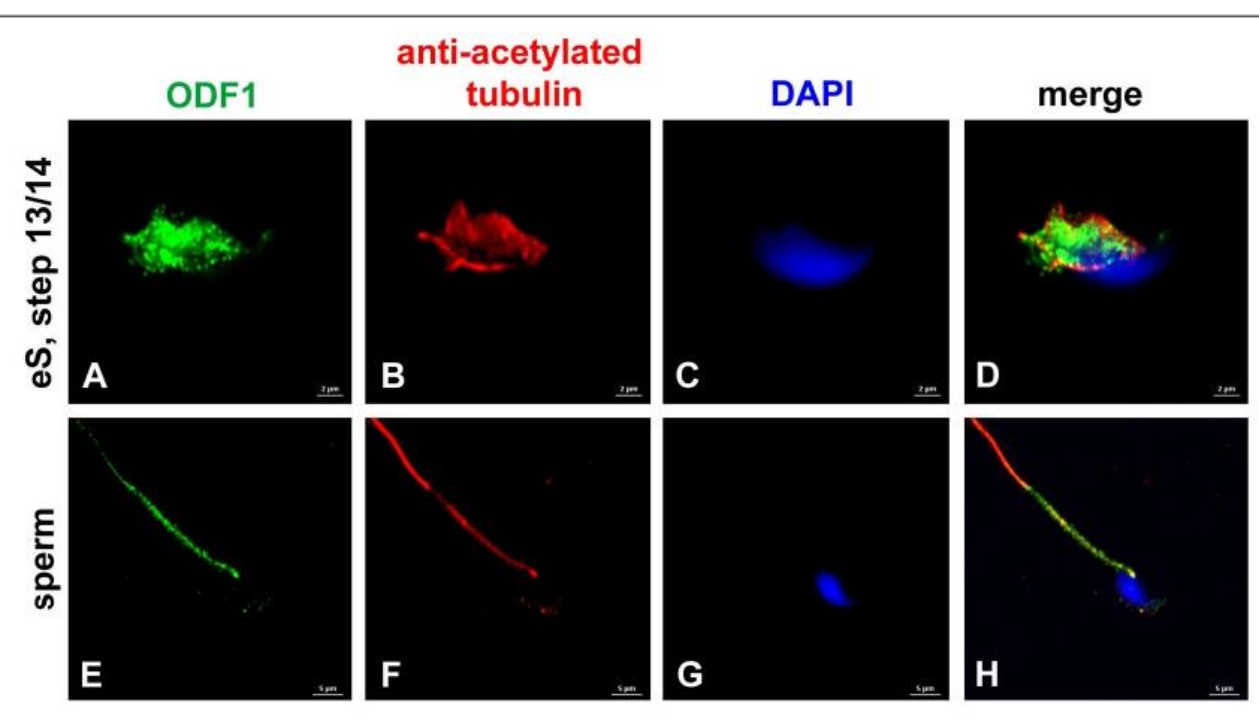

FIGURE $4 \mid$ ODF1 locates to the manchette in elongating spermatids and to the sperm tail. Incubation of mouse testicular cells with antibodies against ODF1 (green) and acetylated tubulin (red). ODF1 decorates the manchette in elongating spermatids (A-D) and the sperm tail (E-H). Nuclear counterstain with DAPI (blue). Bars: $2 \mu \mathrm{m}$ (A-D), $5 \mu \mathrm{m}$ (E-H).

inhibitors (Halt Protease Inhibitor Cocktail 100x, Thermo Fisher Scientific, \#78438) and $0.2 \mathrm{mM}$ PMSF). For in vitro interaction, 6xHis-ODF2 proteins were added to the washed Ni-NTA agarose and incubated for $1 \mathrm{~h}$ at $4^{\circ} \mathrm{C}$ in constant agitation. NIH3T3 cell lysate containing CCDC42-Cherry proteins was split into halves and one half added to the resin followed by incubation for $2 \mathrm{~h}$ at $4^{\circ} \mathrm{C}$ and constant agitation. The second half was used for the negative control. The beads were washed four times in wash buffer, followed by a final overnight washing step. To elute bond proteins, Ni-NTA agarose was incubated for $10 \mathrm{~min}$ at $4^{\circ} \mathrm{C}$ in elution buffer $(50 \mathrm{mM} \mathrm{NaPi}, 500 \mathrm{mM} \mathrm{NaCl}, 500 \mathrm{mM}$ imidazole, $0.2 \mathrm{mM}$ PMSF). Eluates were boiled in SDS-sample buffer and analyzed by Western-blotting using rabbit anti-ODF2 antibodies (ESAP 15572, ABIN2430582, antibodies-online) and rat anti-RedFP antibodies (5F8, Chromotek). As negative control, the resin was incubated with one half of the NIH3T3 cell lysate containing CCDC42-Cherry proteins but without $6 \mathrm{xHis-ODF} 2$ proteins and processed as described.

\section{RESULTS}

\section{CCDC42 Localizes to Manchette,} Perinuclear Ring, Connecting Piece and Sperm Tail

The coiled-coil domain containing protein of $42 \mathrm{kDa}$ (CCDC42) belongs to the DUF4200 family of proteins containing the domain of unknown function 4200. Important paralogs of CCDC42 are CFAP73, CCDC38, and CFAP100, also known as CCDC37/MIA1. CCDC42 orthologs are present in most organisms from vertebrates to choanoflagellates
(Ruan et al., 2008; Guindon et al., 2010). Despite their widespread occurrence, information is currently scarce. $C c d c 42$ is specifically expressed in testis and brain but deletion of $C c d c 42$ seems to affect exclusively male germ cells resulting in infertility (Pasek et al., 2016). Ccdc42-deficient spermatids developed malformed HTCA and sperm tail that are functionally insufficient. However, beyond that no further information about CCDC42 is available. We first studied the subcellular localization of CCDC42 during spermatogenesis in the mouse using a commercially available antibody. The antibody was first validated by immunocytology and Western blotting using the $C c d c 42$-expression construct (validation results are online at antibodies-online and are shown in Figure $9^{1}$ ).

Weak expression of CCDC42 was first observed in the cytoplasm of round spermatids (Figures 1A-D). In elongating spermatids CCDC42 co-localized with the manchette microtubules decorated by acetylated tubulin and more strongly with the perinuclear ring which marks the anterior border of the manchette (Figures 1E-L) Additionally, CCDC42 localized to the connecting piece detectable as two adjacent spots at the posterior end of the nucleus (Figures 1I-L, arrow in J). In sperm, CCDC42, again, was found at the connecting piece region and located to the sperm tail. CCDC42 strongly decorated the principal piece but only weakly the middle piece whereas acetylated tubulin marked the whole tail (Figures 1M-P). Labeling of detached sperm tails confirmed prevalent localization of CCDC42 in the principal piece whereas acetylated tubulin marked the whole tail. However, the anterior region of detached sperm tails, which corresponds to the former attachment site of the tail to the head, showed presence of $\mathrm{CCDC} 42$ visible by

\footnotetext{
${ }^{1}$ https://www.antibodies-online.com
} 


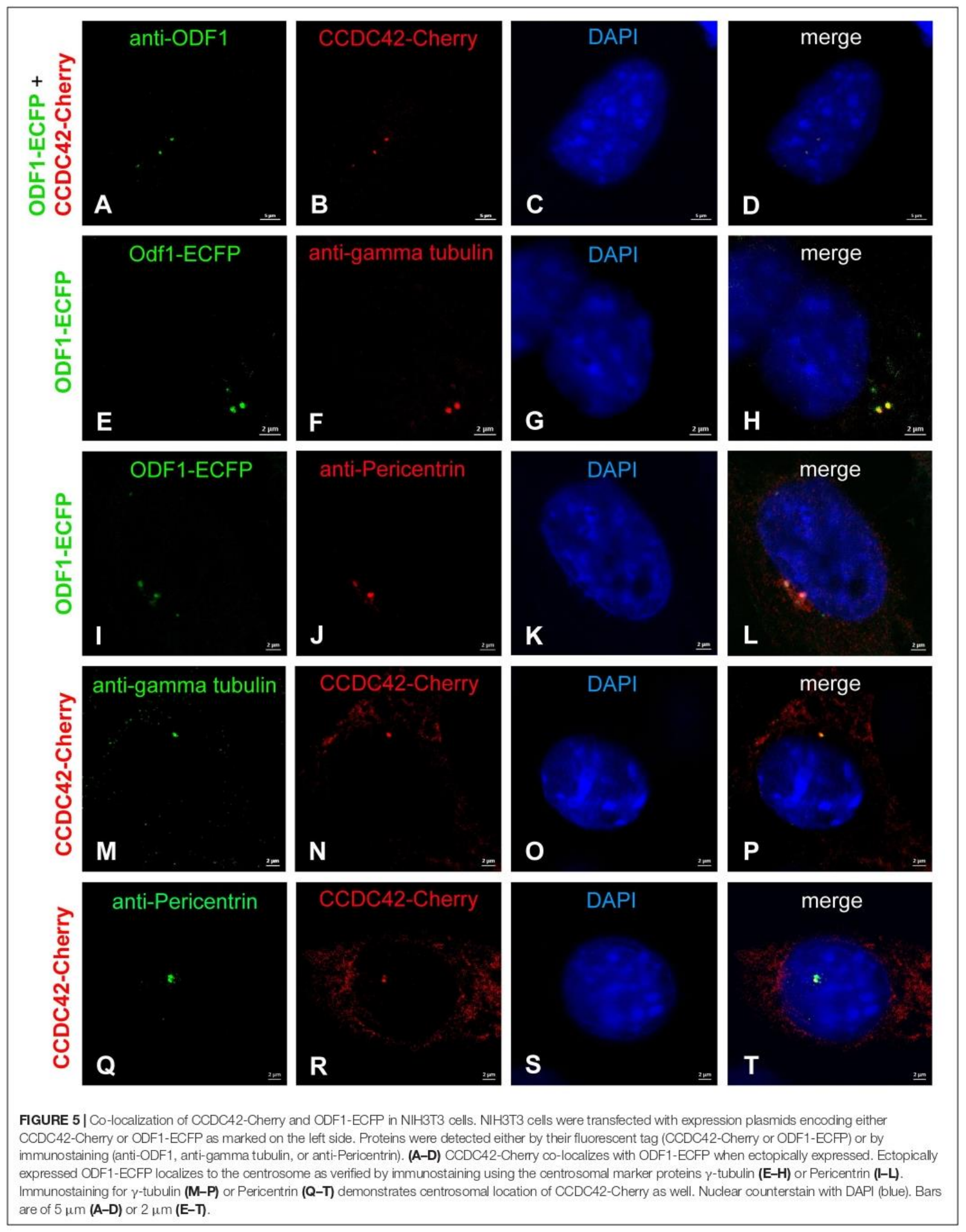



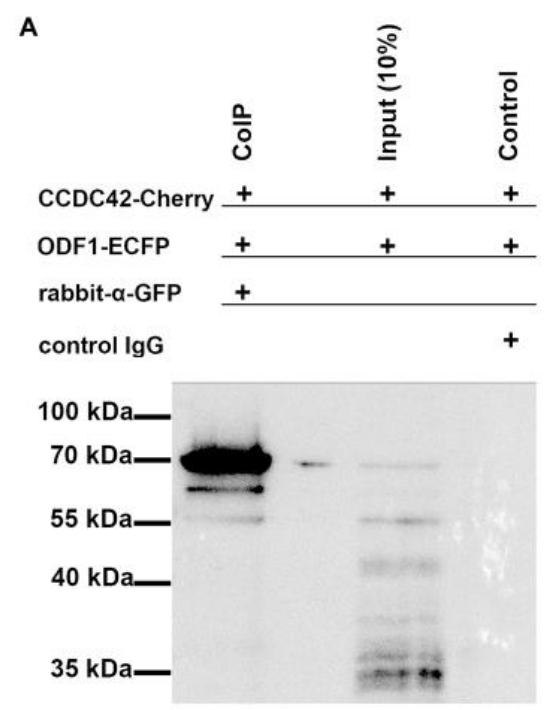

rat anti-RedFP
B
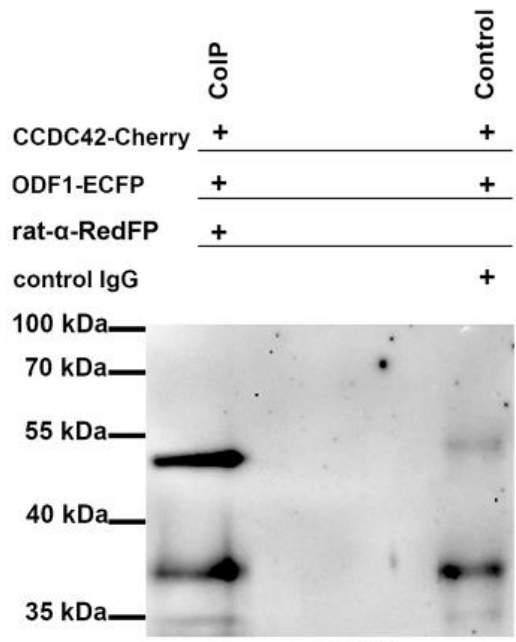

mouse anti-GFP

FIGURE 6 | Co-precipitation of CCDC42 and ODF1. Cells were transfected with the expression plasmids encoding CCDC42-Cherry or ODF1-ECFP. Proteins were immunoprecipitated from cell lysates using either anti-GFP (raised in rabbit, rabbit anti-GFP; $\mathbf{A}$ ) to capture ODF1-ECFP or anti-RedFP (raised in rat, rat anti-RedFP: B) to capture CCDC42-Cherry. The co-purified protein interacting with the captured protein was detected by immunoblotting using either anti-RedFP (A) or anti-GFP (B) as first antibodies followed by detection of first antibodies using the secondary antibodies anti-rat IgG (for detection of CCDC42-Cherry) or anti-mouse IgGs (for detection of ODF1-ECFP). ODF1-ECFP, captured by rabbit anti-GFP antibody, co-precipitated CCDC42-Chemy with an expected molecular mass of $\sim 70 \mathrm{kDa}$.

(A) When CCDC42-Cherry was captured using the rat anti-RedFP-antibody (B), ODF1-ECFP was co-purified as well (B). Immunoblotting using the first antibody against GFP raised in mouse (B), followed by the secondary anti-mouse lgG antibody, detected the expected ODF1-ECFP protein of $\sim 54 \mathrm{kDa}$ but only weakly reacted with the control lgGs, which were raised in rabbit (control) and which show a slightly higher molecular mass.

two adjacent spots (Figures 1Q-T; framed in R, S and enlarged inset in R). A stronger staining of the principal piece of the sperm tail than of the mid-piece might either reflect an unequal distribution of CCDC42 along the sperm tail or is caused by different accessibilities of the antibodies due to the presence of the mitochondrial sheath in the mid-piece.

Decoration of the manchette, the perinuclear ring, and the connecting piece was also demonstrated by using anti$\alpha$-tubulin antibody staining in conjunction with anti-CCDC42, both antibodies subsequently detected by varying secondary antibodies (Figure 2). The control, with anti- $\alpha$-tubulin antibody incubation but omitting anti-CCDC42 antibody, followed by both secondary antibodies subsequently, revealed only $\alpha$-tubulin staining thus supporting specificity of anti-CCDC42 staining (Figures 2I-L).

When concurrently stained for the nuclear envelope protein SUN4, the weak CCDC42 staining of the cytoplasm in round and early elongating spermatids was confirmed (Figures 3AH). The SUN4 positive domain partially overlapped with the CCDC42 positive region as this is the region where the manchette develops. In early elongating spermatids, the SUN4 and CCDC42 localization domains seemingly overlapped corresponding most likely to the region where the manchette has formed and to which SUN4 locates (I-L) (Yang et al., 2018a). Again, CCDC42 more strongly decorated the perinuclear ring (I-L). The weak cytoplasmic staining for CCDC42 in the round spermatid seemed to be beyond background staining as demonstrated by the control experiment (Figures 3M-O).

\section{CCDC42 Interacts With ODF1 and ODF2}

The small heat shock protein ODF1/HSPB10 is a main protein component of the sperm tail ODFs. Beyond that, it locates to the connecting piece and is essential for the tight connection between head and tail (Schalles et al., 1998; Yang et al., 2012, 2014). Immunocytological inspection confirmed sperm tail location of ODF1 and, additionally, showed expression in the manchette of elongating spermatids (Figure 4). Location of ODF1 thus resembled that of CCDC42 raising the question whether both proteins also physically interact.

We first investigated whether the location of ODF1 and CCDC42 is interdependent when ectopically expressed in NIH3T3 mouse fibroblasts. Cells were transfected with expression plasmids encoding either CCDC42 fused to Cherry (CCDC42-Cherry) or ODF1 fused to ECFP (ODF1-ECFP) and the proteins detected either by their fluorescent tags or by immunostaining (Figure 5). Ectopic expression of CCDC42-Cherry revealed bright staining of one or two dots close to the nucleus that overlap with ODF1-ECFP expression (Figures 5A-D). Since twin-dots are a typical signature of the centrosome, we verified centrosomal location of ODF1-ECFP (Figures 5E-L) as well as of CCDC42Cherry (Figures $\mathbf{5 M}-\mathbf{T}$ ) using immunostaining for the 


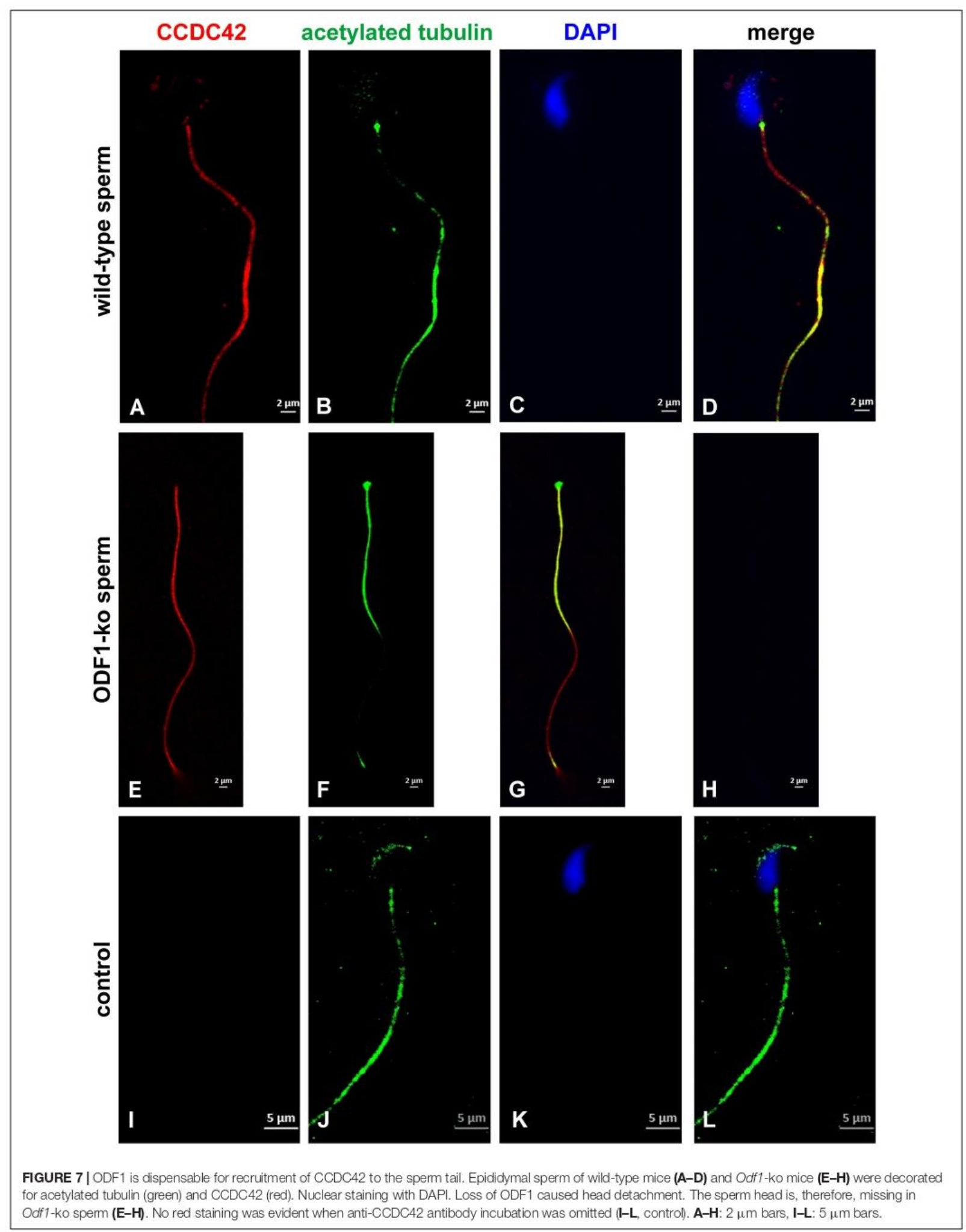




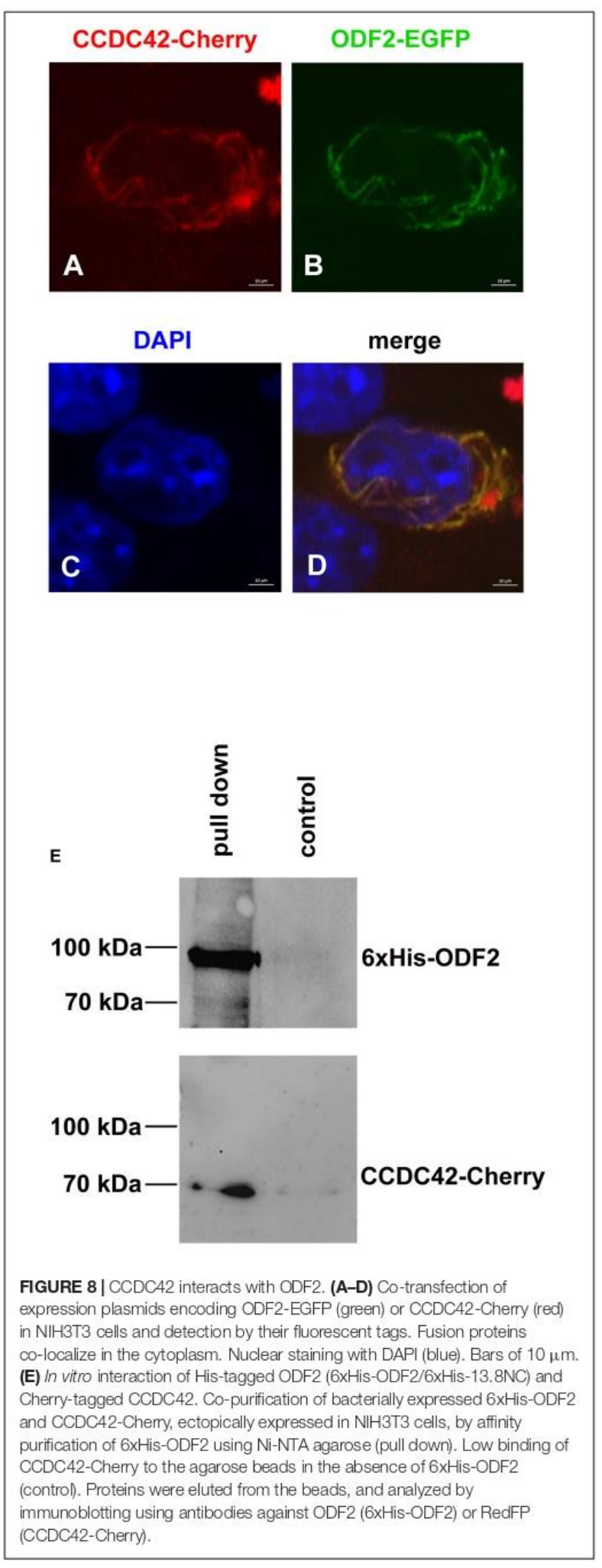

centrosomal marker proteins $\gamma$-tubulin (Figures 5E-H,MP) or Pericentrin (Figures 5I-L,Q-T) (Doxsey et al., 1994). Our results show that CCDC42-Cherry co-localizes with ODF1-ECFP, when both proteins are ectopically expressed in NIH3T3 cells, notably at the centrosome. However, recruitment of CCDC42-Cherry to the centrosome is independent of ODF1 due to the fact that ODF1 is not at all expressed in somatic cells and CCDC42 locates to the centrosome despite absence of ODF1-ECFP (Figures 5M-T) (Yang et al., 2012).

Nevertheless, the physical interaction between CCDC42-Cherry and ODF1-ECFP was proven by coimmunoprecipitation. Both proteins were ectopically expressed in cultured cells by transient transfection of expression plasmids, and one of either protein immunoprecipitated out of the cell lysate. The immunoprecipitate was analyzed by immunoblotting detecting the protein that co-precipitated with the fished protein (Figure 6). Co-immunoprecipitation of CCDC42-Cherry and ODF1-ECFP was verified in either direction, capturing either CCDC42-Cherry or ODF1-ECFP. Our data thus indicate physical interaction between CCDC42 and ODF1. However, ODF1 is not only dispensable for recruitment of $\mathrm{CCDC} 42$ to the centrosome but also to the sperm tail. Immunocytology on epididymal sperm of Odf1-ko mice showed decoration for CCDC42 similar as in wild-type sperm (Figure 7, wild-type sperm in A-D, Odf1ko sperm in E-H). Acetylated tubulin staining identified the sperm tail. As ODF1 is essential for the anchorage of the sperm head to the tail causing head detachment when missing, the sperm head is absent in ODF1-ko sperm (Figures 7E-H) (Yang et al., 2012). In the control staining, when omitting anti-CCDC42 incubation but subsequent incubation with both secondary antibodies, the sperm tail is clearly visible by acetylated tubulin decoration but missed any red staining (Figures 7I-L).

Another important protein of the sperm tail and the connecting piece is ODF2. ODF2, furthermore, is an essential component of the centrosome and the basal body in somatic cells (Brohmann et al., 1997; Schalles et al., 1998; Nakagawa et al., 2001; Hüber et al., 2008). We, therefore, asked whether ODF2 is another binding partner of CCDC42. Co-transfection assays of expression plasmids in NIH3T3 cells revealed similar location of ODF2-EGFP and CCDC42-Cherry. ODF2 fused to EGFP (13.8NC-EGFP) often generated fibrous structures to which CCDC42-Cherry proteins localize (Figures 8A-D). Additionally, a physical interaction between CCDC42-Cherry and ODF2 was proven by pull-down assays (Figure 8E). Bacterially expressed and refolded 6xHis-tagged ODF2 was affinity purified using Ni-NTA agarose in the presence of CCDC42-Cherry, ectopically expressed in cell culture. Thereafter, eluates were immunoblotted for detection of the target protein $6 \mathrm{xHis}-\mathrm{ODF} 2$ and its putative binding partner CCDC42-Cherry. CCDC42-Cherry co-purified with 6xHis-ODF2 (Figure 8E, pull down), whereas almost no binding of CCDC42-Cherry to the beads was observed in the absence of 6xHis-ODF2 (Figure 8E control). Our data, therefore, indicate ODF2 as another binding partner of CCDC42. 

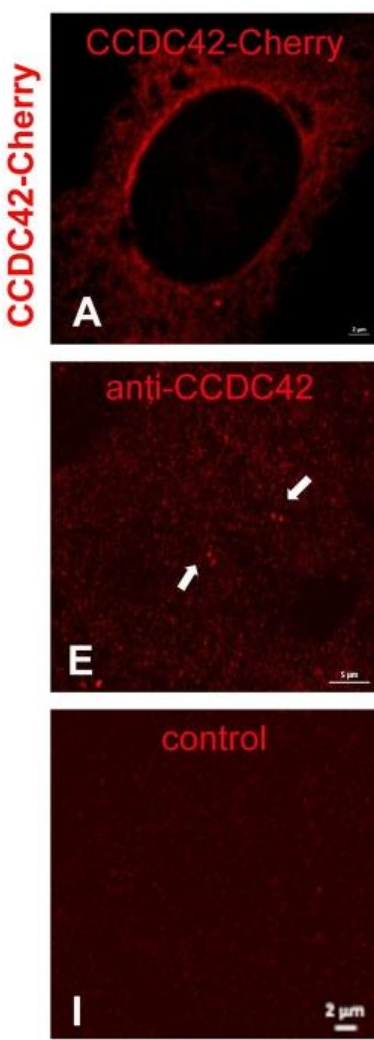
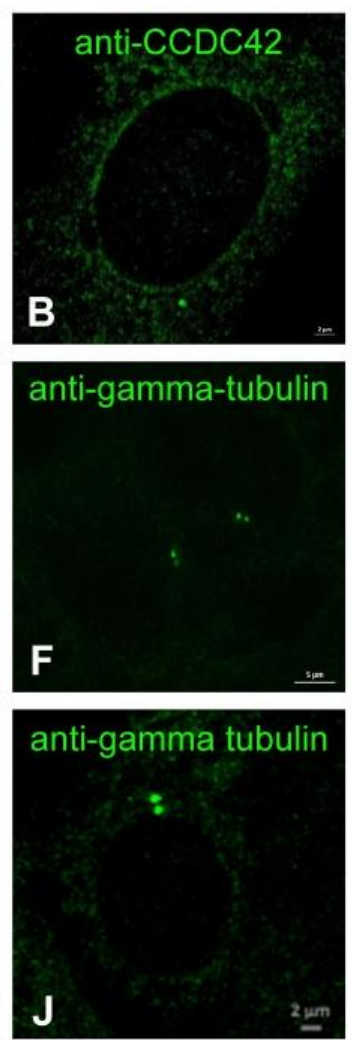
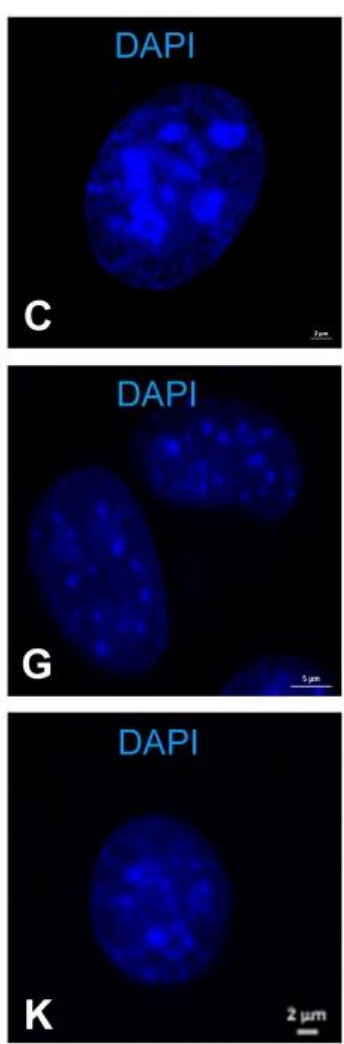
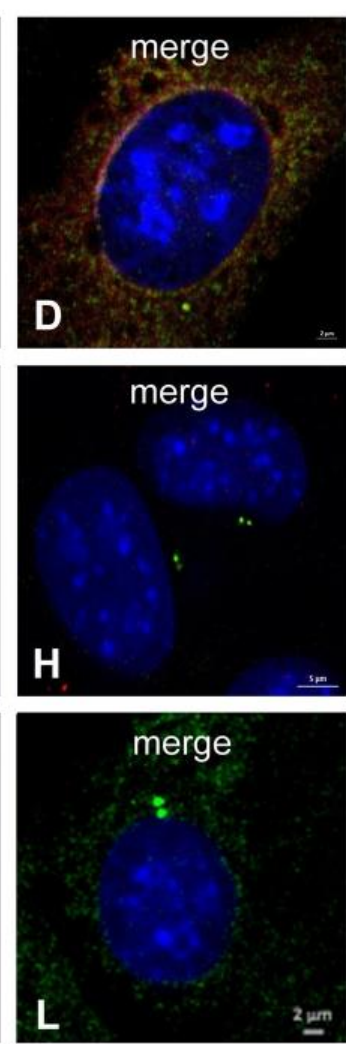

FIGURE 9 | CCDC42 is a centrosomal component in NIH3T3 cells. (A-D) NIH3T3 cells were transfected with Ccdc42-Cherry expression plasmid (as indicated on the left side by $C C D C 42-C h e r r y)$ and $C C D C 42$ detected by its fluorescent $\operatorname{tag}$ (CCDC42-Chern, red) and immuno-decoration (anti-CCDC42, green) to validate the antibody. (E-H) Endogenous expression of CCDC42 in NIH3T3 cells was detected immunocytologically (anti-CCDC42, red) and co-located with the centrosomal marker protein $\gamma$-tubulin (anti-gamma-tubulin, green). (I-L) Omitting anti-CCDC42 antibody incubation showed no red decoration of the centrosome, which was otherwise detected by anti- $\gamma$-tubulin staining (anti-gamma-tubulin, green), demonstrating anti-CCDC42 antibody specificity. Nuclear counterstain with DAPI (blue). Bars are of $2 \mu \mathrm{m}(\mathbf{A}-\mathbf{D}, \mathbf{I}-\mathbf{L})$ or of $5 \mu \mathrm{m}(\mathbf{E}-\mathbf{H})$

\section{CCDC42 Is a Centrosomal Protein in Somatic Cells}

By ectopic expression of CCDC42-Cherry in NIH3T3 cells we have observed a predominant centrosomal location (Figure 5). This prompted us to investigate whether CCDC42 is endogenously expressed in somatic cells being a novel component of the centrosome. We first transfected cells with the CCDC42-Cherry expression plasmid for validation of the antiCCDC42 antibody (Figures 9A-D). The antibody specifically decorated only transfected cells and co-localized with the fusion protein CCDC42-Cherry thus demonstrating its validity (Figures 9A-D). We next incubated untransfected NIH3T3 cells with the anti-CCDC42 antibody. Immunostaining of the endogenous CCDC42 decorated a twin-spot near the nucleus that additionally stained for the centrosomal marker $\gamma$-tubulin (Figures 9E-H). The centrosome was exclusively decorated by $\gamma$-tubulin staining but did not show a red fluorescence when omitting anti-CCDC42 antibody incubation (Figures 9I-L, control). Immunocytological data thus indicate expression of
CCDC42 in somatic cells, which is contradictory to its reported restricted expression pattern.

\section{Expression of Ccdc42 Isoforms}

According to Pasek et al. (2016), Ccdc42 is expressed in testis and brain. In mouse testes, weak expression was first observed at 10-days of age that raised in 15-days old testis and maintained into adulthood. Testicular expression thus corresponds with the onset of meiosis around day 10 and its increase roughly coincides with the progression of spermatid differentiation during spermiogenesis (Nebel et al., 1961). However, three putative CCDC42 isoforms have been reported in mice (UniProtKB - Q5SV66) produced by alternative splicing (Figure 10). The longest isoform 203 (Q5SV66) consists of 316 amino acids (aa). In isoform 201 (Q5SV65) the sequence encoded by exon 5 is missing resulting in a putative protein of 238 aa. Isoform 202 (Q5SV66-2) has a postulated length of 169 aa since the $\mathrm{N}$-terminal end encoded by exons 1-4 is completely missing. Instead, translation of the protein starts at the $3^{\prime}$ end of 


\section{>203_Q5SV66 MSLGMMEEED LAEYFRLQYG ERLLQLLQKF PNVEEQSDSP SIQLLEKKKE AKIMQEAMEH \\ >201_Q5SV65 MMEEED LAEYFRLQYG ERLLQLLQKF PNVEEQSDSP SIQLLEKKKE AKIMQEAMEH}

>203_Q5SV66 KKEAFQRRME TLNLRWEELG IKEEQLKAHI QKFEQFIQEN DQKRIRALKK ANKERELKRL
>201_Q5SV65 KKEAFQRRME TLNLRWEELG IKEEQLKAHI QKFEQFIQEN DQKRIRALKK ANKERELKRL

>203_Q5SV66 RLRELAKAKQ EMMALRLEHQ KLSVKLQDYA IFNKYLEKVV ENSE--:-0.--

>201_Q5SV65 RLRELAKAKQ EMMALRLEHQ KLSVKLQDYA IFNKYLEKVVENSE-_-

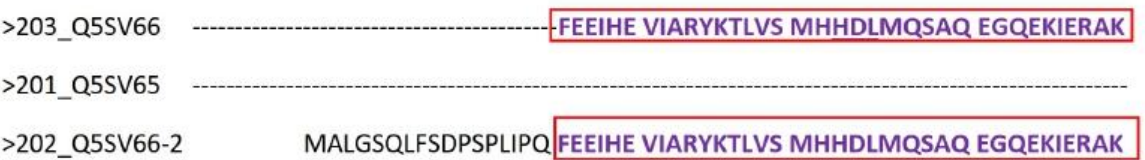

>203_Q5SV66 ARLARYMEEK DDEILQHNNE LARLQMRFDR ARSDVIFWES RWAHIQNTAA KKTLLLGTIK

>201_Q5SV65 ES RWAHIQNTAA KKTLLLGTIK

>202_Q5SV66-2 ARLARYMEEKDDEILQHNNE LARLQMRFDR ARSDVIFWES RWAHIQNTAA KKTLLLGTIK

$\begin{array}{lll}\text { >203_Q5SV66 } & \text { MATLNLFQIV SKQLKESTQV SLEDTHKQLD MIQQFIQDLS DIWTEVKKKE QQQVRM } & 316 \text { aa } \\ \text { >201_Q5SV65 } & \text { MATLNLFQIV SKQLKESTQV SLEDTHKQLD MIQQFIQDLS DIWTEVKK KEQQQVRM } & 238 \text { aa } \\ \text { >202_Q5SV66-2 } & \text { MATLNLFQIV SKQLKESTQV SLEDTHKQLD MIQQFIQDLS DIWTEVKK KEQQQVRM } & 169 \text { aa }\end{array}$

FIGURE 10|Amino acid alignment of reported CCDC42 isoforms. The longest isoform 203 (Q5SV66) consists of 316 aa. In isoform 201 (Q5SV65) the sequence encoded by exon 5 is missing resulting in a putative protein of 238 aa. Isoform 202 (Q5SV66-2) has a postulated length of 169 aa since the N-terminal end encoded by exons 1-4 is completely missing. Instead, translation of the protein starts at the $3^{\prime}$ end of intron 4 encoding sequence MALGSQLFSDPSPLIPQ. The domain of unknown function 4200 (DUF4200), comprising aa 44-161 at the N-terminal half of isoform 203, is depicted in violet. The coiled-coil region is highlighted in yellow. The epitope, to which antibody ABIN2785068 is directed, is framed in red.

intron 4 encoding sequence MALGSQLFSDPSPLIPQ upstream of exon 5 encoded sequences. According to Interpro, the domain of unknown function 4200 (DUF4200) comprises aa 44-161 at the N-terminal half of isoform 203 and is therefore present in both, isoform 203 as well as in isoform 201, albeit shortened in the latter, but is largely missing in isoform 202 (Figure 10, depicted in violet). The coiled-coil region at the C-terminal end is present in all three isoforms (according to SMART; in Figure 10 highlighted in yellow). The antibody ABIN2785068, used for immunocytology, detects the epitope of 50 aa in the DUF4200, which is present in isoforms 203 and 202 but not in 201 (Figure 10, enframed in red).

Expression of $\mathrm{Ccdc} 42$ was reported to be restricted to testis. In somatic tissues, with the only exception being the brain, $C c d c 42$ seems to be not expressed. We confirmed testicular expression by RT-PCR using different primer combinations (Figure 11). The full-length product of isoform Ccdc42-203 was expected to be of
1093 bp, which was confirmed by RT-PCR (Figure 11A, exons 1-7). However, for the putative isoform Ccdc42-201, a length of 729 bp was expected since exon 5 was skipped but we could not amplify the expected fragment (Figure 11A). Amplification of $305 \mathrm{bp}$ of the $5^{\prime}$ region (exons $1+2$ ) again confirmed testicular expression of $\mathrm{Ccdc} 42$ (Figure 11A, exons $1+2$ ). When amplifying 203 bp of the conserved $3^{\prime}$ region, Ccdc42 expression was also demonstrated in NIH3T3 mouse fibroblasts (Figure 11A, exons 6+7).

Our results thus indicate that expression of $\mathrm{Ccdc42}$ isoforms is not restricted to testis. This prompted us to revise $C c d c 42$ expression in other tissues (Figure 11B). We performed a nested PCR in order to detect even low expression levels. The first RTPCR was performed to amplify exons 5-7 encoding part of the DUF domain with the epitope detected by the antibody, and the conserved coiled-coil region. We found strong expression in testis and epididymides and weak expression in the brain. 


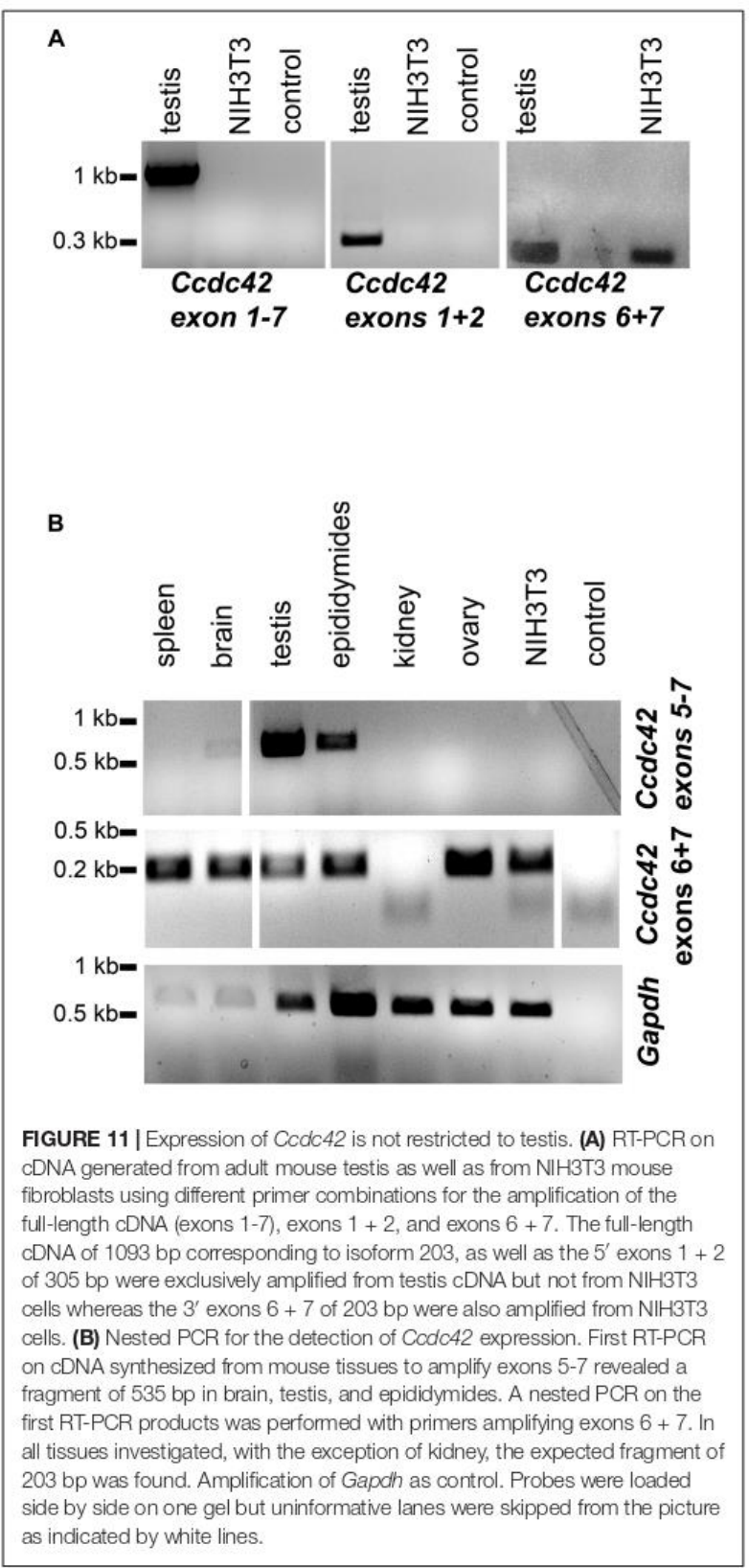

Sequencing of the RT-PCR products generated form testis and epididymides confirmed Ccdc42. Gapdh amplification, albeit demonstrating successful cDNA synthesis in all tissues, also indicated low amounts of cDNA in spleen and brain, which most likely accounts for the weak RT-PCR band found in brain CDNA. When performing a nested PCR on the first PCR products by amplifying exons $6+7$ a fragment of the expected size was found in all tissues, with the exception of kidney. The RT-PCR fragments generated in ovary and NIH3T3 cDNA were sequenced confirming $C c d c 42$ amplification. Our results, therefore, indicate that $C c d c 42$ expression is not restricted to testis. However, since the full-length product and the $5^{\prime}$ region (exons $1+2$ ) were found only in testis but not in NIH3T3 cells it is most likely that in somatic cells another isoform than the full-length CCDC42 isoform 203 is predominantly expressed, and that this isoform was detected by immunocytology in the centrosome.

\section{DISCUSSION}

The HTCA is a complex structure present in the neck region of the sperm interconnecting the head and the tail. It develops from the centrosome that itself is composed of a pair of centrioles and associated components. During spermiogenesis, the proximal centriole inserts into a nuclear indentation, known as the implantation fossa, opposed to the acrosomal cap, and the linkage complex and the longitudinal columns of the connecting piece are formed (Fawcett and Phillips, 1969). The sperm tail starts outgrowing from the distal centriole of the former centriole that is now the basal body. In order to transmit only one centriole during fertilization, sperm of most vertebrates have disintegrated the distal centriole leaving only the proximal centriole. In contrast to centriole reduction in most vertebrates, in mice and other rodents, both distal and proximal centrioles degenerate during spermiogenesis leaving the centriolar vaults (Schatten, 1994; Hoyer-Fender, 2011). The current dogma of centrosome reduction in sperm was recently revisited by investigating the centrosomal protein inventory in human sperm. These data showed that the distal centriole is remodeled into an atypical centriole surrounded by a pericentriolar matrix instead of being completely vanished (Fishman et al., 2018). It is, therefore, feasible to view the neck structure as a specialized form of the pericentriolar matrix, and the centrioles as nucleation site for both the sperm tail and the manchette MTs. Few proteins are currently known that affect the head to tail linkage when missing, in between ODF1 and CCDC42 (Yang et al., 2012; Pasek et al., 2016). ODF1 is located in the sperm tail ODFs and in the connecting piece (Schalles et al., 1998). It interacts with ODF2, the major outer dense fiber protein, but no further interacting proteins have been confirmed (Shao et al., 1997). To figure out the interrelationship of HTCA proteins, and how they function in the formation of the HTCA, ODF1 interacting proteins are of utmost importance.

The coiled-coil domain containing 42 protein CCDC42 is highly conserved in evolution with orthologs existing in most organisms from vertebrates to choanoflagellates (Ruan et al., 2008; Guindon et al., 2010). Additionally, important paralogs of CCDC42 (also named CCDC42A) exist as CFAP73 (also named CCDC42B and MIA2), CCDC38 and CFAP100 (also named CCDC37 and MIA1). They altogether constitute the CFAP73 protein family. These proteins are in essential coiled-coil domain proteins and share the domain of unknown function DUF4200. Coiled-coil domain containing proteins are often involved in ciliary motility (Inaba and Mizuno, 2016; Zur Lage et al., 2019). In $C$. reinhardtii the gene product of the CCDC42 homolog MIA2 is a dynein regulator and necessary for ciliary motility 
(Yamamoto et al., 2013). Further coiled-coil domain proteins as CCDC39 and CCDC40 are essential for ciliary motility by assembly of the dynein regulatory complex (Becker-Heck et al., 2011; Merveille et al., 2011; Blanchon et al., 2012). Ccdc42-ko mice are phenotypically normal but males are sterile. Sterility of $C c d c 42$-deficient male mice is most likely caused by the malformation of the HTCA and the sperm tail resulting in functional insufficiency (Pasek et al., 2016). These data suggest that CCDC42 has an important function in male germ cells but is otherwise dispensable (Pasek et al. 2016). Since CCDC42-deficiency affected exclusively the male germ cell, CCDC42 seems not to be involved in ciliary motility because otherwise, a more generalized phenotype has to expected. CCDC42-deficient spermatids are characterized by a multiplicity of the HTCA, defective nuclear shaping despite presence of a manchette, dislocation of the HTCA from its implantation site and a loss of flagellar outgrowth from the HTCA (Pasek et al., 2016).

We have demonstrated by immunocytology that CCDC42 $\mathrm{Co}-$ localizes with ODF1- and ODF2-fusion proteins when ectopically expressed, and with structures comprising these proteins endogenously. Furthermore, co-precipitation by pull-down and co-immunoprecipitation experiments indicated binding of CCDC42 to ODF1 as well as to ODF2. However, recruitment of CCDC42 to the sperm tail and the centrosome in somatic cells does not require ODF1. CCDC42 consists in essential of coiled-coil domains, which is also the main feature of ODF2. Coiled-coil domains are important oligomerization domains mediating homodimerization as well as heterodimerization (Mason and Arndt, 2004). It is, therefore, most likely that ODF2 and CCDC42 interact by means of their coiled-coil domains. This mutual interaction might contribute to the stabilization of important cytoskeletal structures potentially mediated by ODF1. Although the true molecular function of ODF1 is not known, it belongs to the small heat shock protein family and might, therefore, act as a chaperone in protein folding (Fontaine et al., 2003). The protein complex consisting of the core proteins ODF2/ODF1/CCDC42 may thus build the rigid scaffold essential for the formation of the connecting piece and the sperm tail. When missing any one of these proteins the rigid scaffold is damaged causing failure of the linkage complex and the sperm tail. Whether CCDC42 interacts with any of those proteins that have a reported function in HTCA formation, as Centrin 1, Centrobin, Spata6, or Azil/Cep131 awaits further investigation (Liska et al., 2009; Avashti et al., 2013; Hall et al., 2013; Yuan et al., 2015). However, since CCDC42-deficient sperm often show two instead of one basal body inserted into the nuclear membrane, centriole duplication, as well as correct attachment of the centrioles to the implantation fossa seem to be affected (Pasek et al., 2016). As similar phenotypes have been observed concerning mutations in Centrobin, Centrin 1, and Azi/Cep131 a functional interaction with CCDC42 is likely (Liska et al. 2009; Avashti et al., 2013; Hall et al., 2013). Our observation that CCDC42 is expressed in the manchette and in particular in the perinuclear ring illuminates its involvement in the acrosome-acroplaxome complex formation and nuclear shaping that are both affected when CCDC42 is missing. Since loss of CCDC42 did not prevent manchette formation, it is most likely involved in stabilizing the manchette or is a passenger protein transported via the manchette. Its accumulation in the perinuclear ring, however, points toward a stabilizing function and its involvement in the attachment of the manchette to the nuclear membrane.

Our data show that CCDC42 expression is not restricted to testis and brain but instead is found also in somatic tissues. We detected the endogenous protein in the centrosome of somatic NIH3T3 cells and hence identified CCDC42 as a novel component of the centrosome and the sperm tail not found before by large scale proteomics screens (Amaral et al., 2013; centrosome database Centrosome:DB). However, albeit RT-PCR experiments confirmed expression of $\mathrm{Ccdc} 42$ in somatic tissues, the full-length sequence could only by amplified from testis cDNA. It is therefore probable that the full-length isoform 203 is restricted to testis or more specifically to male germ cells whereas another isoform is expressed in somatic tissues. We could not verify expression of isoform 202, which starts with translated sequences encoded by intron 4 since a primer that binds to these $5^{\prime}$ sequences has amplified an unrelated almost unknown sequence (C6H1orf158). Furthermore, we got no indications by RT-PCR of isoform 201, which was expected to be encoded by a smaller cDNA due to skipping of exon 5 . Our data additionally show that CCDC42 is a component of the centrosome in somatic cells and most likely functions in scaffolding the centrosome via interaction with ODF2/Cenexin. However, since the only obvious phenotype of CCDC42-deficient mice is male infertility, CCDC42 is either dispensable for the somatic centrosome or its function has been taken over by other members of the CFAP73 family.

\section{DATA AVAILABILITY}

The datasets generated for this study are available on request to the corresponding author.

\section{AUTHOR CONTRIBUTIONS}

CTC did the experiments and prepared the figures. SH-F was the project leader and wrote the manuscript. Both authors read and approved the final manuscript.

\section{FUNDING}

CTC got a Ph.D. grant of the DAAD/CONICYT BECAS Chile program.

\section{ACKNOWLEDGMENTS}

We thank Manfred Alsheimer (Würzburg) for the kind gift of $\alpha$-SUN4 antibody. 


\section{REFERENCES}

Akhmanova, A., Mausset-Bonnefont, A. L., van Cappellen, W., Keijzer, N., Hoogenraad, C. C., Stepanova, T., et al. (2005). The microtubule plus-endtracking protein CLIP-170 associates with the spermatid manchette amd is essential for spermatogenesis. Genes Dev. 19, 2501-2515. doi: 10.1101/gad. 344505

Amaral, A., Castillo, J., Estanyol, J. M., Ballesca, J. L., Ramalho-Santos, J., and Oliva, R. (2013). Human sperm proteome suggests new endogenous metabolic pathways. Mol. Cell. Proteomics 12, 330-342. doi: 10.1074/mcp.M1 12.020552

Avashti, P., Scheel, J. F., Ying, G., Frederick, J. M., Baehr, W., and Wolfrum, U. (2013). Germline deletion of Cetnl causes infertility in male mice. J. Cell Sci. 126, 3204-3213. doi: $10.1242 /$ jcs. 128587

Baltz, J. M., Williams, P. O., and Cone, R. A. (1990). Dense fibers protect mammalian sperm against damage. Biol. Reprod. 43, 485-491. doi: 10.1095/ biolreprod43.3.485

Becker-Heck, A., Zohn, I. E., Okabe, N., Pollock, A., Lenhart, K. B., Sullivan-Brown, J., et al. (2011). The coiled-coil domain containing protein CCDC40 is essential for motile cilia function and left-right axis formation. Nat. Genet. 43, 79-84 doi: $10.1038 / \mathrm{ng} .727$

Blanchon, S., Legendre, M., Copin, B., Duquesnoy, P., Montantin, G., Kott, E., et al. (2012). Delineation of CCDC39/CCDC40 mutation spectrum and associated phenotypes in primary ciliary dyskinesia. J. Med. Genet. 49, 410-416. doi: 10. 1136/jmedgenet-2012-100867

Brohmann, H., Pinnecke, S., and Hoyer-Fender, S. (1997). Identification and characterization of new cDNAs encoding outer dense fiber proteins of rat sperm. J. Biol. Chem. 272, 10327-10332. doi: 10.1074/jbc.272.15.10327

Burfeind, P., and Hoyer-Fender, S. (1991). Sequence and developmental expression of a mRNA encoding a putative protein of rat sperm outer dense fibers. Dev. Biol. 148, 195-204. doi: 10.1016/0012-1606(91)90329-2

Calvi, A., Wong, A. S., Wright, G., Wong, E. S., Loo, T. H., Stewart, C. L., et al. (2015). SUN4 is essential for nuclear remodelling during mammalian spermiogenesis. Dev. Biol. 407, 321-330. doi: 10.1016/j.ydbio.2015. 09.010

Chen, S. R., Batool, A., Wang, Y. Q., Hao, X. X., Chang, C. S., Cheng, C. Y., et al. (2016). The control of male fertility by spermatid-specific factors: searching for contraceptive targets from spermatozoon's head to tail. Cell Death Dis. 7:e2472. doi: $10.1038 /$ cddis.2016.344

Clermont, Y., Oko, R., and Hermo, L. (1993). "Cell biology of mammalian spermiogenesis," in Cell and Molecular Biology of the Testis, eds C. Desjardins and L. Ewing (New York, NY: Oxford University Press), 332-376.

Donkor, F. F., Mönnich, M., Czirr, E., Hollemann, T., and Hoyer-Fender, S. (2004). Outer dense fibre protein 2 (ODF2) is a self-interacting centrosomal protein with affinity for microtubules. J. Cell. Sci. 117, 4643-4651. doi: 10.1242/jcs. 01303

Dooher, G. B., and Bennett, D. (1973). Fine structural observations on the development of the sperm head in the mouse. Am. J. Anat. 136, 339-362.

Doxsey, S. J., Stein, P., Evans, L., Calarco, P. D., and Kirschner, M. (1994). Pericentrin, a highly conserved centrosome protein involved in microtubule organization. Cell 76, 639-650. doi: 10.1016/0092-8674(94)90504-5

Fawcett, D. W. (1975). The mammalian spermatozoon. Dev. Biol. 44, 394-436. doi: 10.1016/0012-1606(75)90411-x

Fawcett, D. W., Anderson, W. A., and Phillips, D. M. (1971). Morphogenetic factors influencing the shape of the sperm head. Dev. Biol. 26, 220-251. doi: 10.1016/0012-1606(71)90124-2

Fawcett, D. W., and Phillips, D. M. (1969). The fine structure and development of the neck region of the mammalian spermatozoon. Anat. Rec. 165, 153-164

Fishman, E. L., Jo, K., Nguyen, Q. P. H., Kong, D., Royfma, N. R., Cekic, A. R., et al. (2018). A novel atypical sperm centriole is functional during human fertilization. Nat. Commun. 9:2210.

Fontaine, J.-M., Rest, J. S., Welsh, M. J., and Benndorf, R. (2003). The sperm outer dense fiber protein is the 10 th member of the superfamily of mammalian small stress proteins. Cell Stress Chaperones 8, 62-69.

Guindon, S., Dufayard, J.-F., Lefort, V., Anisimova, M., Hordijk, W., and Gascuel, O. (2010). New algorithms and methods to estimate maximum-likelihood phylogenies: assessing the performance of PhyML 3.0. Syst. Biol. 59, 307-321. doi: $10.1093 /$ sysbio/syq010
Hall, E. A., Keighren, M., Ford, M. J., Davey, T., Jarman, A. P., Smith, L. B., et al (2013). Acute versus chronic loss of mammalian Azil/Cep131 results in distinct ciliary phenotypes. PLoS Genet. 9:e 1003928. doi: 10.1371/journal.pgen. 1003928

Hoyer-Fender, S. (2011). "Centrosomes in fertilization, early embryonic development, stem cell division, and cancer," in Deep Insight for The Atlas of Genetics \& Cytogenetics in Oncology and Haematology, ed. J. L. Huret (Vandæuvre-lès-Nancy: Institute of Scientific and Technical).

Hüber, D., Geisler, S., Monecke, S., and Hoyer-Fender, S. (2008). Molecular dissection of ODF $2 /$ Cenexin revealed a short stretch of amino acids necessary for targeting to the centrosome and the primary cilium. Eur. J. Cell Biol. 87 , 137-146. doi: 10.1016/j.ejcb.2007.10.004

Inaba, K., and Mizuno, K. (2016). Sperm dysfunction and ciliopathy. Reprod. Med. Biol. 15, 77-94. doi: 10.1007/s12522-015-0225-5

Jan, S. Z., Hamer, G., Repping, S., de Rooij, D. G., van Pelt, A. M. M., and Vormer T. L. (2012). Molecular control of rodent spermatogenesis. Biochim. Biophys. Acta 1822, 1838-1850. doi: 10.1016/j.bbadis.2012.02.008

Kierszenbaum, A. L. (2001). Spermatid manchette: plugging proteins to zero into the sperm tail. Mol. Reprod. Dev. 59, 347-349. doi: $10.1002 / \mathrm{mrd} .1040$

Kierszenbaum, A. L. (2002). Intramanchette transport (IMT): managing the making of the spermatid head, centrosome, and tail. Mol. Reprod. Dev. 63, 1-4. doi: $10.1002 / \mathrm{mrd} .10179$

Kierszenbaum, A. L., Rivkin, E., and Tres, L. L. (2011). Cytoskeletal track selection during cargo transport in spermatids is relevant to male fertility. Spermatogenesis 1, 221-230. doi: 10.4161/spmg.1.3.18018

Laemmli, U. K. (1970). Cleavage of structural proteins during assembly of the head of the bacteriophage T4. Nature 227, 680-685. doi: 10.1038/227680a0

Lehti, M. S., and Sironen, A. (2016). Formation and function of the manchette and flagellum during spermatogenesis. Reproduction 151, R43-R54. doi: 10.1530/ REP-15-0310

Lehti, M. S., and Sironen, A. (2017). Formation and function of sperm tail structures in association with sperm motility defects. Biol. Reprod. 97, 522-536. doi: 10.1093/biolre/iox096

Lindemann, C. B. (1996). Functional significance of the outer dense fibers of mammalian sperm examined by computer simulation with the geometric clutch model. Cell. Motil. Cytoskelet. 34, 258-270. doi: 10.1002/(sici)1097-0169(1996) $34: 4<258$ ::aid- $\mathrm{cm} 1>3.0 . \mathrm{co} ; 2-4$

Liska, F., Gosele, C., Rivkin, E., Tres, L. L., Cardoso, M. C., Domaing, P., et al (2009). Rat hd mutation reveals an essential role of centrobin in spermatid head shaping and assembly of the head-tail coupling apparatus. Biol. Reprod. 81,1196-1205. doi: 10.1095/biolreprod.109.078980

Mason, J. M., and Arndt, K. M. (2004). Coiled coil domains: stability, specificity, and biological implications. Chembiochem 5, 170-176. doi: 10.1002/cbic. 200300781

Mendoza-Lujambio, I., Burfeind, P., Dixkens, C., Meinhardt, A., Hoyer-Fender S., and Neesen, J. (2002). The Hookl gene is non-functional in the abnormal spermatozoon head shape (azh) mutant mouse. Hum. Mol. Genet. 11, 16471658. doi: $10.1093 / \mathrm{hmg} / 11.14 .1647$

Merveille, A. C., Davis, E. E., Becker-Heck, A., Legendre, M., Amirav, I., Bataille, G., et al. (2011). CCDC39 is required for assembly of inner dynein arms and the dynein regulatory complex and for normal ciliary motility in humans and dogs. Nat. Genet. 43, 72-78. doi: 10.1038/ng.726

Nakagawa, Y., Yamane, Y., Okanoue, T., Tsukita, S., and Tsukita, S. (2001). Outer dense fiber 2 is a widespread centrosome scaffold component preferentially associated with mother centrioles: its identification from isolated centrosomes. Mol. Biol. Cell 12, 1687-1697. doi: 10.1091/mbc.12.6.1687

Nebel, B. R., Amarose, A. P., and Hackett, E. M. (1961). Calendar of gametogenic development in the prepuberal male mouse. Science 134, 832-833. doi: 10.1126/ science.134.3482.832

O'Donnell, L., and O'Bryan, M. K. (2014). Microtubules and spermatogenesis Semin. Cell. Dev. Biol. 30, 45-54. doi: 10.1016/j.semcdb.2014.01.003

Pasch, E., Link, J., Beck, C., Scheuerle, S., and Alsheimer, M. (2015). The LINC complex component Sun4 plays a crucial role in sperm head formation and fertility. Biol. Open 4, 1792-1802. doi: 10.1242/bio.015768

Pasek, R. C., Malarkey, E., Berbari, N. F., Sharma, N., Kesterson, R. A., Tres, L. L. et al. (2016). Coiled-coil domain containing 42 (Ccdc42) is necessary for proper sperm development and male fertility in the mouse. Dev. Biol. 412, 208-218. doi: 10.1016/j.ydbio.2016.01.042 
Rattner, J. B., and Brinkley, B. R. (1972). Ultrastructure of mammalian spermiogenesis. III. The organization and morphogenesis of the manchette during rodent spermatogenesis. J. Ultrastruc. Res. 41, 209-218. doi: 10.1016/ s0022-5320(72) 90065-2

Rattner, J. B., and Olson, G. (1973). Observations on the fine structure of the nuclear ring of the mammalian spermatid. J. Ultrastruc. Res. 43, 438-444. doi: $10.1016 / \mathrm{s} 0022-5320(73) 90020-8$

Ruan, J., Li, H., Chen, Z., Coghlan, A., Coin, L. J. M., Guo, Y., et al. (2008). TreeFam: 2008 Update. Nucleic Acids Res. 36, D735-D740.

Russell, L. D., Ettlin, R. A., Sinha Hikim, A. P., and Clegg, E. D. (1990). Histological and Histopathological Evaluation of the Testis. Clearwater, FL: Cache River Press, 120. doi: $10.1111 /$ j.1365-2605.1993.tb01156.x

Russell, L. D., Russell, J. A., MacGregor, G. R., and Meistrich, M. L. (1991). Linkage of manchette microtubules to the nuclear envelope and observations of the role of the manchette in nuclear shaping during spermiogenesis in rodents. Am. J. Anat. 192, 97-120. doi: 10.1002/aja.1001920202

Schalles, U., Shao, X., van der Hoom, F., and Oko, R. (1998). Developmental expression of the $84-\mathrm{kDa}$ ODF sperm protein: localization to both the cortex and medulla of outer dense fibers and to the connecting piece. Dev. Biol. 199, 250-260. doi: 10.1006/dbio. 1998.8931

Schatten, G. (1994). The centrosome and its mode of inheritance: the reduction of the centrosome during gametogenesis and its restoration during fertilization. Dev. Biol. 165, 299-335. doi: 10.1006/dbio.1994.1256

Shao, X., Tarnasky, H. A., Schalles, U., Oko, R., and van der Hoorn, F. (1997). Interactional cloning of th $84-\mathrm{kDa}$ major outer dense fiber protein Odf84. Leucine zippers mediate associations of Odf84 and Odf27. J. Biol. Chem. 272, 6105-6113. doi: $10.1074 / j b c .272 .10 .6105$

Towbin, H., Staehelin, T., and Gordon, J. (1979). Electrophoretic transfer of proteins from polyacrylamide gels to nitrocellulose sheets: procedure and some applications. Proc. Natl. Acad. Sci. U.S.A. 76, 4350-4354. doi: 10.1073/pnas.76. 9.4350

Wolosewick, J. J., and Bryan, J. H. D. (1977). Ultrastructural characterization of the manchette microtubule in the seminiferous epithelium of the mouse. Am. J. Anat. 150, 301-332.

Yamamoto, R., Song, K., Yanagisawa, H. A., Fox, L., Yagi, T., Wirschell, M., et al. (2013). The MIA complex is a conserved and novel dynein regulator essential for normal ciliary motility. J. Cell Biol. 201, 263-278. doi: 10.1083/jcb. 201211048

Yang, K., Adham, I. A., Meinhardt, A., and Hoyer-Fender, S. (2018a). Ultrastructure of the sperm head-to-tail linkage complex in the absence of the spermatid-specific LINC component SPAG4. Histochem. Cell Biol. 150, 49-59. doi: 10.1007/s00418-018-1669-7

Yang, K., Tylkowski, M. A., Hüber, D., Tapia Contreras, C., and HoyerFender, S. (2018b). ODF2/Cenexin maintains centrosome cohesion by restricting ß-catenin accumulation. J. Cell Sci. 131:jcs220954. doi: 10.1242/jcs.22 0954

Yang, K., Grzmil, P., Meinhardt, A., and Hoyer-Fender, S. (2014). Haplo-deficiency of ODF1/HSPB10 in mouse sperm causes relaxation of head-to-tail linkage. Reproduction 148, 499-506. doi: 10.1530/REP-14-0370

Yang, K., Meinhardt, A., Zhang, B., Grzmil, P., Adham, I. M., and Hoyer-Fender, S. (2012). The small heat shock protein ODF 1/HSPB 10 is essential for tight linkage of sperm head to tail and male fertility in mice. Mol. Cell. Biol. 32, 216-225. doi: 10.1128/MCB.06158-11

Yuan, S., Stratton, C. J., Bao, J., Zheng, H., Bhetwal, B. P., Yanagimachi, R., et al. (2015). Spata6 is required for normal assembly of the sperm connecting piece and tight head-tail junction. Proc. Natl. Acad. Sci. U.S.A. 112, E430-E439. doi: $10.1073 /$ pnas. 1424648112

Zur Lage, P., Newton, F. G., and Jarman, A. P. (2019). Survey of the ciliary motility machinery of Drosophila sperm and ciliated mechanosensory neurons reveals unexpected cell-type specific variations: a model for motile ciliopathies. Front. Genet. 10:24. doi: 10.3389/fgene.2019.00024

Conflict of Interest Statement: The authors declare that the research was conducted in the absence of any commercial or financial relationships that could be construed as a potential conflict of interest.

Copyright $\odot 2019$ Tapia Contreras and Hoyer-Fender. This is an open-access article distributed under the terms of the Creative Commons Attribution License (CC BY). The use, distribution or reproduction in other forums is permitted, provided the original author(s) and the copyright owner(s) are credited and that the original publication in this journal is cited, in accordance with accepted academic practice. No use, distribution or reproduction is permitted which does not comply with these terms. 


\subsubsection{The WD40-protein CFAP52/WDR16 is a centrosome/basal body protein and localizes to the manchette and the flagellum in male germ cells}

This chapter reports the cilia and flagella-associated protein 52, CFAP52, as a new structural component of male germ cells located at the manchette and sperm tail. Initially, CFAP52 was related to the presence of motile cilia, while in Chlamydomonas, it contributes to the stabilization of the axoneme. Cfap52 transcription has been detected in testes and the ependymal layer of the brain. However, the subcellular localization of CFAP52 had not been described. Thus, we have investigated CFAP52 localization in somatic and male germ cells. Here, we show that CFAP52 localizes to the transient manchette in round and elongating spermatids and the sperm tail. Further, our results reveal that CFAP52 expression is not restricted to male germ cells but instead that CFAP52 is a novel centrosome/basal body protein that also resides in the mitotic spindle poles and the mid-body. Thus, these data indicate that CFAP52 is associated with a subset of microtubular structures, especially centrosome/centriole derived structures.

Beyond that, CFAP52 is a member of the WD-repeat proteins, which mediate protein interactions. Therefore, CFAP52 might likewise act in the assembly or stabilization of protein complexes constituting the centrosomal/basal body matrix, the manchette, and the sperm tail. Furthermore, the location of CFAP52 to the sperm tail but not to the axoneme of primary cilia implies that CFAP52 fulfills specific functions in the stability or maintenance of the sperm tail.

Constanza Tapia Contreras and Sigrid Hoyer-Fender

Author Contributions: Conceived and designed experiments: SH-F. Performed experiments: CTC. Prepared figures and wrote the manuscript: SH-F and CTC. Both authors read and approved the final manuscript.

Status of manuscript: Published in Scientific Reports in 2020. 

is a centrosome/basal body protein and localizes to the manchette and the flagellum in male germ cells

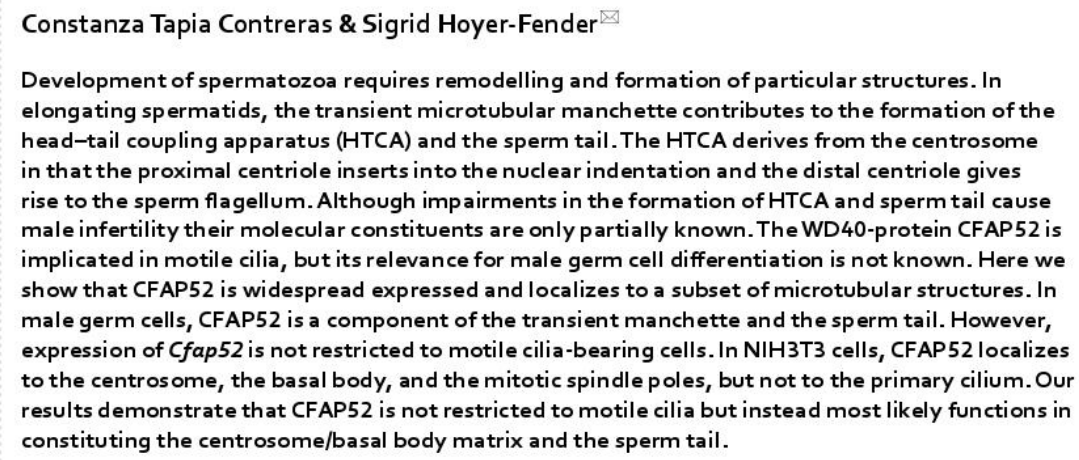

Development of spermatozoa requires remodelling and formation of particular structures. In elongating spermatids, the transient microtubular manchette contributes to the formation of the head-tail coupling apparatus (HTCA) and the sperm tail. The HTCA derives from the centrosome in that the proximal centriole inserts into the nuclear indentation and the distal centriole gives rise to the sperm flagellum. Although impairments in the formation of $\mathrm{HTCA}$ and sperm tail cause male infertility their molecular constituents are only partially known. The WD40-protein CFAP52 is implicated in motile cilia, but its relevance for male germ cell differentiation is not known. Here we show that CFAP52 is widespread expressed and localizes to a subset of microtubular structures. In male germ cells, CFAP52 is a component of the transient manchette and the sperm tail. However, expression of $C$ fap 52 is not restricted to motile cilia-bearing cells. In NIH3T3 cells, CFAP 52 localizes to the centrosome, the basal body, and the mitotic spindle poles, but not to the primary cilium. Our results demonstrate that CFAP52 is not restricted to motile cilia but instead most likely functions in constituting the centrosome/basal body matrix and the sperm tail.

The cilia and flagella associated protein 52, CFAP52 (also named WDR16, WD repeat domain 16, WDRPUH, FLJ37528) is a member of the large WD40-repeat protein family and is widely expressed in eukaryotes. WD40repeats, which are also known as WD or beta-transducin repeats, are short motifs of 40-60 amino acids often terminating in the dipeptide WD. The WD40-domain often comprises several of the WD40-repeats each forming a beta-pleated sheet in a propeller blade $e^{1,2}$. WD 40 proteins are crucially involved in several physiological functions as diverse as signal transduction, RNA processing, remodeling the cytoskeleton, regulation of vesicular traffic, and cell cycle control ${ }^{3-5}$. Their immediate purpose most likely is to serve as platforms for the assembly of protein complexes and to mediate protein-protein interactions ${ }^{6}$.

The WD40 protein family member WDR16 is highly conserved having homologs in invertebrates and vertebrates including flies and humans 7 . In rat, expression of WDR16 was mainly observed in testis and in ependymal cells of the brain, and with reduced expression in lungs. Its expression profile, especially during ependymal development, indicated a correlation to the presence of motile cilia whereas in cells harboring primary cilia WDR16 could not be detected? ${ }^{7}$. By immunofluorescence, WDR16 was found in the cytosol of rat testicular cells, especially in early spermatocytes and pachytene spermatocytes showing a uniform and unspecific localization. Sperm flagella stained negative for WDR16 but in Western blots the tail fraction of bull sperm revealed WDR16. Overall, these results indicated that WDR16 is a marker for motile cilia-bearing cells. WDR16 depletion in zebrafish caused hydrocephalus, however, ciliary movement remained intact. Similarly, the ependymal layer did not display visible alterations ${ }^{7}$. WDR16, furthermore, seems to be involved in the establishment of the left-right symmetry in humans as the homozygous deletion caused laterality disorders ${ }^{8}$. Taken together, these data indicated, that WDR16 is functionally associated with motile cilia, either in the ependym or in the embryonic node albeit cilia

Johann-Friedrich-Blumenbach-Institute of Zoology and Anthropology - Developmental Biology, GZMB, Ernst-Caspari-Haus, Justus-Von-Liebig-Weg11, Georg-August-Universität Göttingen, 37077 Göttingen, Germany. email: shoyer@gwdg.de 
seem not to be directly affected. Nevertheless, WDR16 might be involved in the stabilization of microtubular structures, as observed for the Chlamydomonas WDR16 ortholog FAP52 that plays an important role in axoneme stability ${ }^{9}$. Our intention was therefore, to elucidate the association of WDR16 with cilia in more detail by investigating its expression and localization at the subcellular level. We were interested in both motile and immotile cilia/flagella firstly taking advantage of flagella formation in mammalian spermatogenesis.

Haploid spermatids derived from the second meiotic division differentiated into functional spermatozoa in spermiogenesis. This process is characterized by reshaping of spermatids including elongation and condensation of the nucleus, and the development of specific structures as the acrosome, the transient manchette and the flagellum, and eventually the shedding of the cytoplasm ${ }^{10,11}$. These morphological changes produce highly polarized cells determined by the apical acrosome and the flagellum at the opposite pole. The functionality of the spermatozoon, that is propelling the sperm nucleus towards the oocyte, depends on the tight linkage between sperm tail and nucleus mediated by the head-to-tail coupling apparatus (HTCA) ${ }^{10}$. The HTCA and its morphological equivalent the connecting piece develops from the centrioles. During spermiogenesis, the acrosome and the paired centrioles migrate to opposite poles of the nucleus ${ }^{12}$. The proximal centriole inserts into the caudal area of the nucleus forming the implantation fossa whereas the distal centriole constitutes the basal body that gives rise to the axoneme $e^{10,13,14}$. The sperm flagellum, furthermore, contains additional so-called accessory structures as the outer dense fibres (ODFs) that went laterally to the microtubule (MT) doublets of the axoneme and provide elasticity and stiffness to the flagellum ${ }^{15,16}$

Spermatid elongation is characterized by the formation of a transient microtubular structure, the manchette. The manchette MTs are connected to the perinuclear ring, which is closely associated to the acrosomal border, and extend progressively towards the caudal region thus forming a skirt-like structure surrounding the nucleus ${ }^{17,18}$. The manchette is essential for nuclear reshaping and considered as a track for the delivery of cargos to assemble HTCA and sperm tail ${ }^{19,20}$

Besides being far from figured out in detail up to now, knock out mouse models were useful in shedding light on the underlying genetic causes responsible for impaired spermiogenesis and male infertility ${ }^{21-23}$. In this regard, several genes were identified affecting manchette formation and reshaping of the spermatid when mutated, e.g. the abnormal spermatozoon head shape $(a z h)$ phenotype is caused by a mutation in the Hook1 gene $\mathrm{e}^{24-27}$ Hook1-depletion, furthermore, disrupted the tight connection between flagellum and nucleus causing sperm decapitation ${ }^{26}$. Sperm decapitation and male infertility were also observed when the outer dense fibre protein ODF1 or one of its interacting proteins centrosomal protein coiled-coil domain containing 42 (CCDC42) or the testis-specific SUN-domain protein SPAG4L/SUN5 were mutated ${ }^{28-31}$. SUN-domain proteins are integral nuclear membrane proteins and are part of the linker of nucleoskeleton and cytoskeleton (LINC) complex responsible for bridging the nuclear envelope and the cytoskeleton ${ }^{32}$. In contrast, deficiency of SPAG4/SUN4, the second testis-specific SUN-domain protein and reported ODF1-binding protein caused disjunction of the manchette and male infertility but not decapitation ${ }^{33-37}$.

In order to figure out the protein interaction network responsible for HTCA and sperm tail formation we focused here on the cilia and flagella associated protein 52 (CFAP52). We used RT-PCR for expression analyses, and investigated its sub-cellular localization by immunocytology in transfected cells and in male germ cells. We found that CFAP52 is not restricted to motile cilia bearing cells but instead most likely is ubiquitously expressed. Our data indicate that CFAP52 is associated with a subset of MT structures especially centriole/centrosome derived structures, and the manchette and the axoneme in the sperm flagellum.

\section{Results}

Widespread expression of Cfap52. In order to verify the reported expression of Cfap52, we performed RT-PCR using total RNA isolated from mouse tissues, and in addition from the mouse fibroblast cell lin NIH3T3. Amplification of exons 1-14 of Cfap52 revealed the expected fragment of 1871 bp in testis and ovary but not in any of the other probes (Fig. 1a), whereas amplification of Gapdh in all probes verified the quality of cDNAs (Fig. 1c). These results confirmed the previous observation of a testicular expression of Cfap52 but additionally demonstrated that Cfap52 is also highly expressed in ovary, which to the best of our knowledge do not harbor cells with motile cilia. However, to detect even low expression levels we performed a nested PCR on the first PCR product amplifying a region comprising parts of exons 4 to 6 . A DNA fragment of the expected size of $255 \mathrm{bp}$ was found in all samples (Fig. 1b). Sequencing of the PCR products obtained from brain, ovary and epididymis confirmed amplification of Cfap52. The different intensities obtained for the secondary PCR product are caused by differing amounts of first PCR used as template. These results demonstrate that Cfap 52 is expressed in all tissues investigated including the fibroblast cell line. Cfap52, therefore, seems to be ubiquitously expressed, including somatic tissues and cells that to not generate motile cilia, although at a low level. Expression of Cfap52 is therefore not restricted to motile cilia bearing cells.

CFPA52 localizes to the spermatid manchette and to the sperm tail. Testicular expression of Cfap52, previously reported in the rat, was confirmed by our investigation in the mouse. However, besides an overall and unspecific cytoplasmic distribution of CFAP52 in spermatocytes no further specification is currently available ${ }^{7}$. We therefore intended to investigate the subcellular localization of CFAP52 during mouse spermatogenesis using mouse testicular cell suspensions decorated by a commercially available antibody. We first validated the anti-CFAP52 antibody for immunocytology by generating a CFAP52::EGFP fusion construct, transfected the plasmid into NIH3T3 cells, and decorated the expressed fusion protein with the anti-CFAP52 antibody (Fig. 2a-d). Antibody staining was exclusively found in transfected cells, identified by their green autofluorescence of the CFAP52::EGFP fusion. Furthermore, the antibody co-localized with the green fluorescent CFAP52::EGFP fusion protein. 


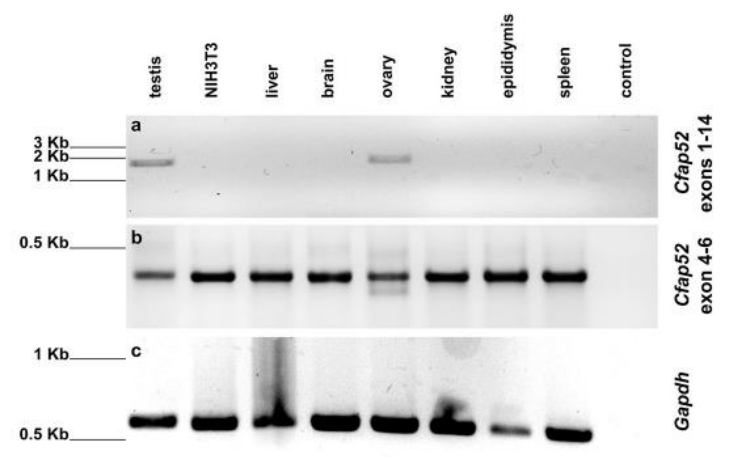

Figure 1. Cfap52 expression is not correlated with the presence of motile cilia. RT-PCR on cDNA synthesized from different mouse tissues and from the fibroblast cell line NIH3T3. (a) Amplification of exon 1 to exon 14 of Cfap52. The expected fragment size of 1871 bp is found in testis and ovary. (b) Nested PCR on the first RT-PCR products was performed to amplify parts of exon 4 to exon 6 of Cfap52. The expected fragment size of $255 \mathrm{bp}$ is present in all probes. (c) Gapdh amplification as quality check. Control is the PCR reaction without template.
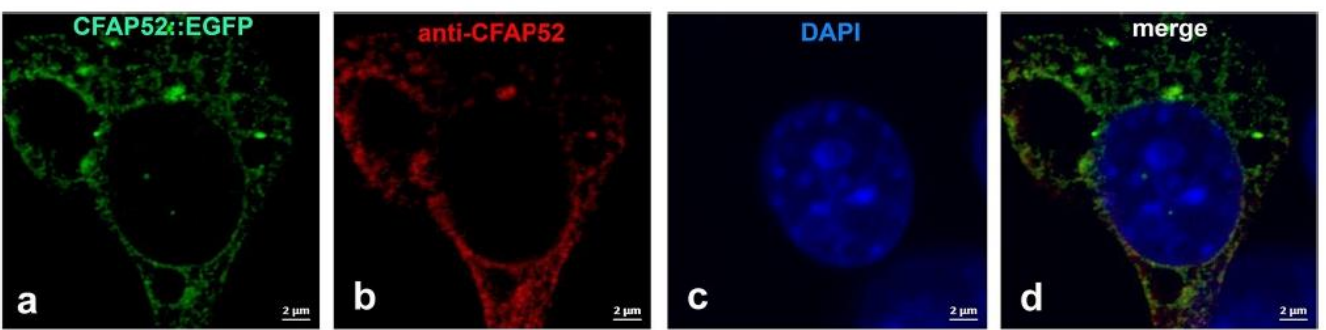

Figure 2. Validation of anti-CFAP52 antibody. NIH3T3 cells were transfected with the $p C f a p 52:: E g f p$ expression plasmid encoding the fusion protein CFAP52::EGFP. Proteins were detected either by their autofluorescence (green) or by the commercial anti-CFAP52 antibody (red). Colocalization of the inherent green fluorescence and the antibody decoration demonstrated antibody specificity (a-d). Nuclear counterstain with DAPI (blue). Bars: $2 \mu \mathrm{m}$

Having the anti-CFAP52 antibody validated, we performed immunocytology on mouse testicular cell suspensions (Fig. 3). The acrosome was decorated by peanut lectin-FITC staining to enable identification of spermatid differentiation steps (Fig. 3). A weak expression of CFAP52 with a few strong dots and a shallow indication of fibrous structures at the nuclear surroundings was first found in early round spermatids (Fig. 3a-d). These weak structures are most likely the first indications of the forming manchette, as fibre like structures with a strong CFAP52 reactivity are present in round spermatids of a more advanced step (Fig. 3e-h). Thereafter, a clear manchette with its characteristic parallel bundles was visible in round spermatids (Fig. 3i-l) and elongating spermatids (Fig. $3 \mathrm{~m}-\mathrm{x}$ ) that is strongly stained for CFAP52 indicating an association of CFAP52 with microtubules. In mature spermatozoa, CFAP52 located to the sperm tail with a somehow stronger intensity in the principal piece than in the mid-piece (Fig. $\left.3 y-y^{\prime \prime \prime}\right)$. No antibody staining was detected in elongating spermatids when anti-CFAP52 antibody was omitted (Fig. 3z-z"').

Colocalization of CFAP52 with the manchette microtubules was demonstrated by alpha-tubulin decoration in round and elongating spermatids (Fig. 4a-h). Furthermore, CFAP52 showed a similar distribution as the LINC components SUN3 and SUN4 (Fig. 4i-p). Enrichment of CFAP52 at the head-to-tail coupling apparatus (HTCA), which was decorated by $\gamma$-tubulin staining, was not found (Fig. 4q-t). Additionally, CFAP52 is not enriched at the perinuclear ring, which was decorated for the microtubule plus-end binding protein EB3 (Fig. 4u-x), but showed a faint ring-like concentration anteriorly to the perinuclear ring (Fig. $4 \mathrm{u}, \mathrm{x}$, arrows) ${ }^{23}$

A similar distribution in the manchette and the sperm tail was observed for the outer dense fibre protein 1 , $O D F 1^{31}$, posing the question whether the observed colocalization indicates interaction or interdependency. We, therefore, investigated CFAP52 localization in testicular sections using both wild-type as well as Odf1-deficient mice $^{28}$. In testis-sections of Odf1 $1^{+/+}$-mice we observed CFAP52-decoration in the manchette (Fig. 5a-d, i-l, arrows). Odf1-deficient testis-sections exhibited the same strong CFAP52-staining in the manchette (Fig. 5e-h, 


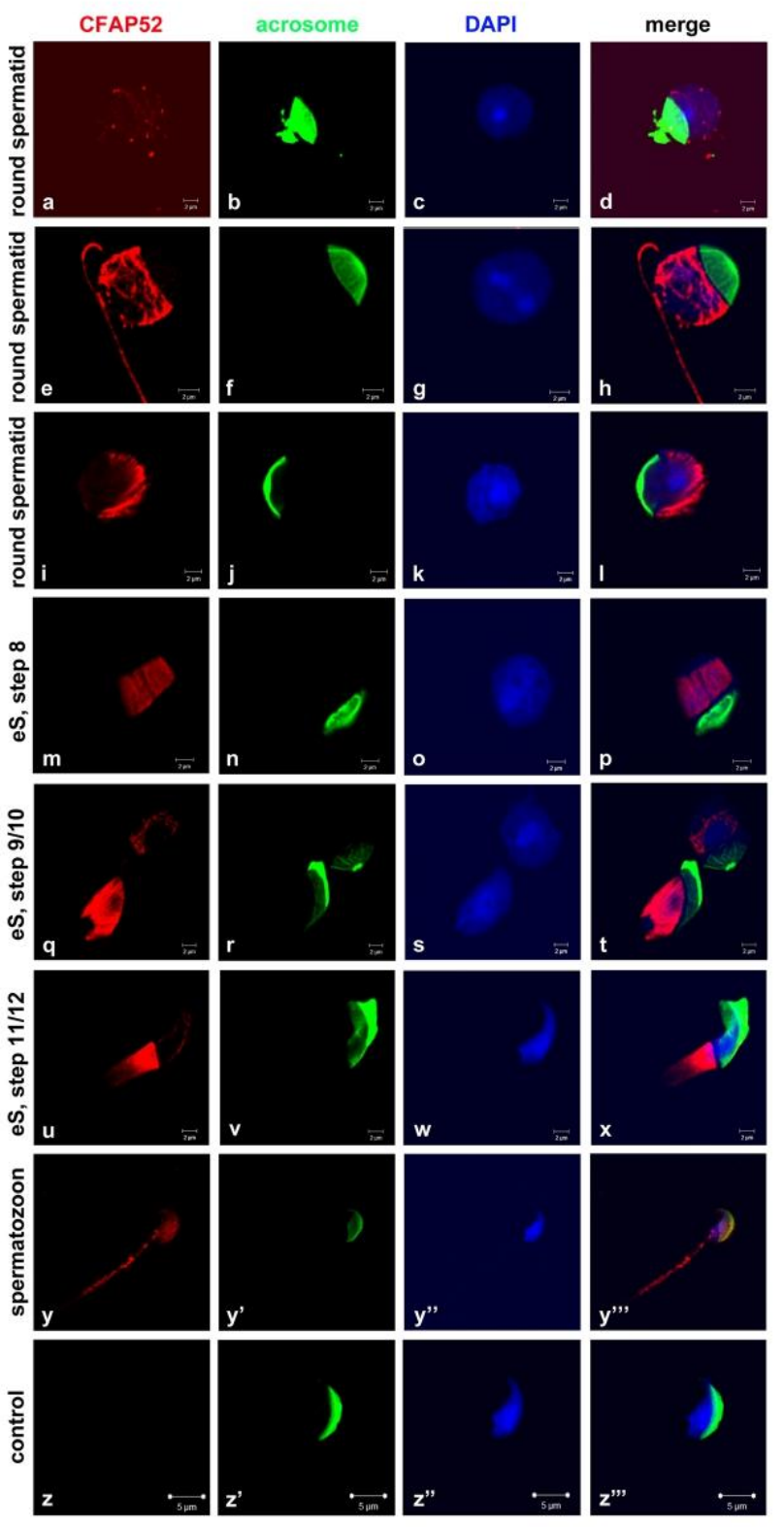

Figure 3. Subcellular localization of CFAP52 in male germ cells. CFAP52 is located to manchette and sperm tail in mouse male germ cells. Suspension preparations of adult mouse testis were incubated with a commercial antibody against CFAP52 (red) and the acrosome decorated with PL-FITC. The developing manchette is decorated with CFAP52 in round and elongating spermatids (a-x). In spermatozoa, CFAP52 locates to the tail with a more intense staining of the principal piece than the mid-piece $\left(\mathbf{z}-\mathbf{z}^{\prime \prime \prime}\right)$. No staining of elongating spermatozoa was evident when the anti-CFAP52 antibody was omitted (control, $\left.\mathbf{y}-\mathbf{y}^{\prime \prime \prime}\right)$. 

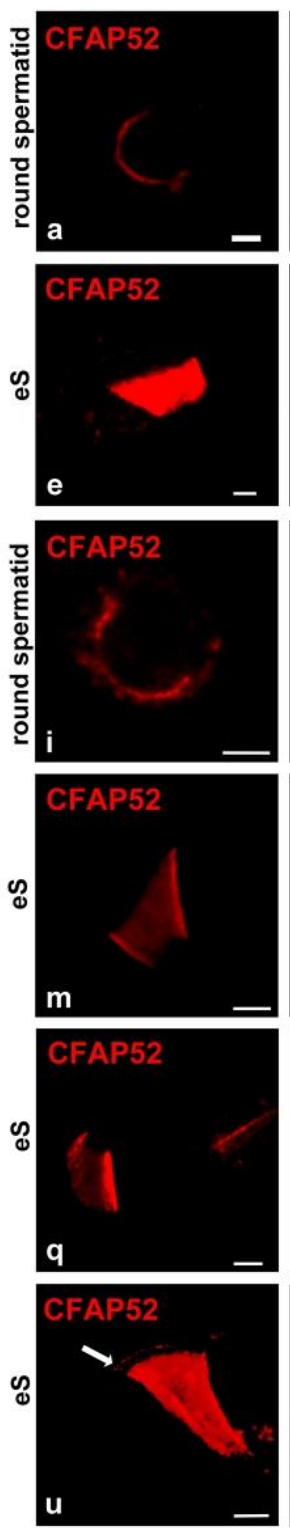

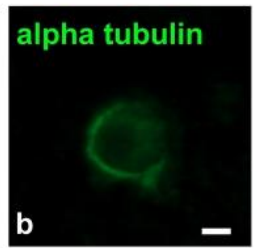

alpha tubulin
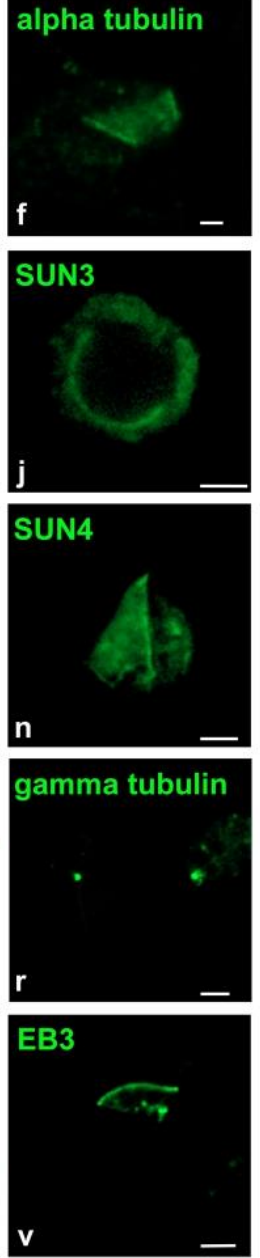

DAPI
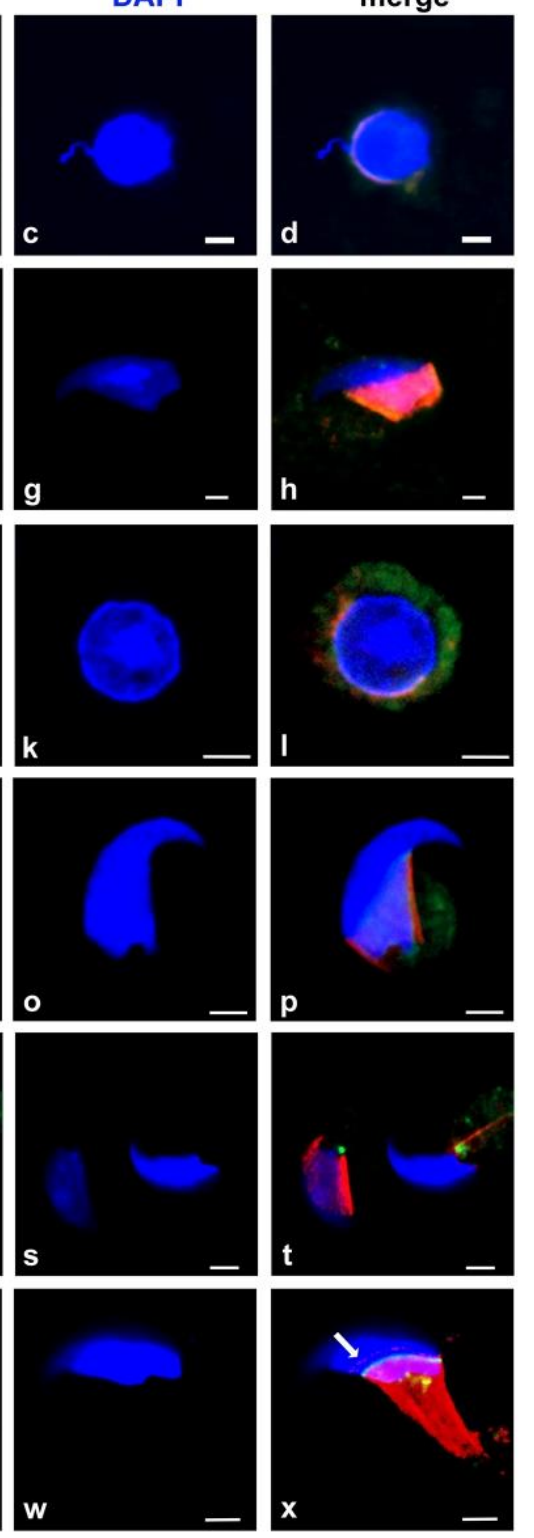

Figure 4. CFAP52 localizes to the manchette but not the perinuclear ring in spermatids. CFAP52 colocalizes with alpha-tubulin in the manchette in round (a-d) and elongating spermatids (e-h), and shows a similar distribution as the LINC components SUN3 (i-l) and SUN4 (m-p). Highlighting the basal body of the sperm tail by staining for gamma-tubulin could not demonstrate enrichment of CFAP52 (q-t). The microtubule plusend tracking protein EB3 decorates the perinuclear ring that is not enriched for CFAP52 (u-x). The arrow points to the faint ring-like concentration of CFAP52 anteriorly to the perinuclear ring. CFAP52 in $\operatorname{red}(\mathbf{a}, \mathbf{e}, \mathbf{i}, \mathbf{m}, \mathbf{q}, \mathbf{u})$, all other antibodies decorated in green $(\mathbf{b}, \mathbf{f}, \mathbf{j}, \mathbf{n}, \mathbf{r}, \mathbf{v})$. Nuclear counterstain with DAPI in blue $(\mathbf{c}, \mathbf{g}, \mathbf{k}, \mathbf{o}, \mathbf{s}, \mathbf{w})$ and merged images $(\mathbf{d}, \mathbf{h}, \mathbf{l}, \mathbf{p}, \mathbf{t}, \mathbf{x})$. eS: elongating spermatids. Bars: $2 \mu \mathrm{m}(\mathbf{a}-\mathbf{h})$ or $2.5 \mu \mathrm{m}(\mathbf{i}-\mathbf{x})$. 

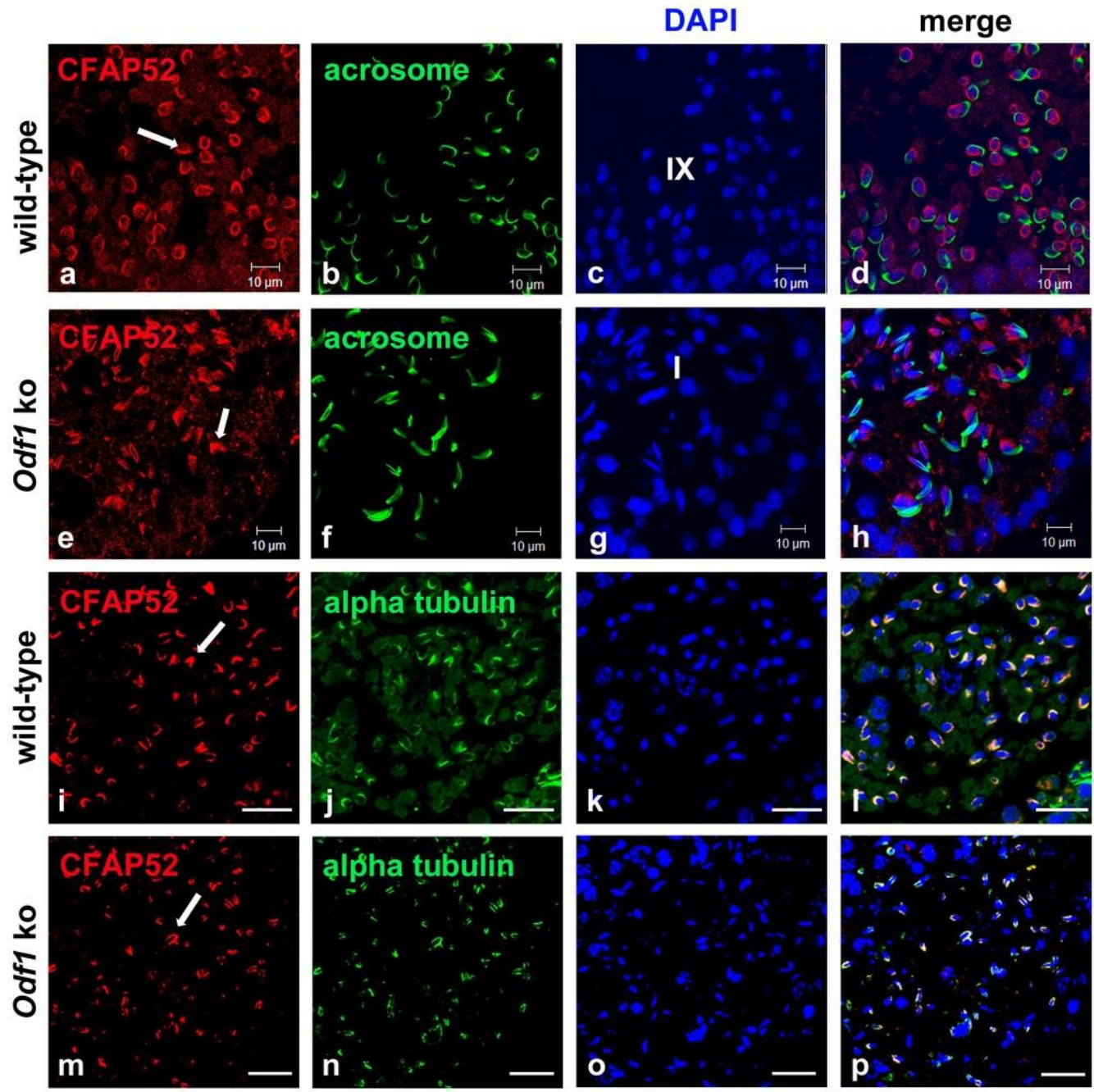

Figure 5. Depletion of ODF1 does not affect manchette location of CFAP52. CFAP52 decorates the manchette (arrows) in wild-type (a-d, $\mathbf{i}-\mathbf{l})$ as well as in Odf1 ko testis sections (e-h, $\mathbf{m}-\mathbf{p}$ ) (in red). (a-h) Decoration of the acrosome by PL-FITC (green). Spermatogenic stages according to the acrosome formation are indicated (stage IX for the wild-type and stage I for the $\operatorname{Odf} 1 \mathrm{ko}$ ). (i-p) Double immunofluorescence with both, antiCFAP52 (red) and anti-alpha-tubulin (green). Nuclear stain with DAPI (blue). (a, e, i, m) anti-CFAP52 antibody decoration (red), (b, f) PL-FITC (green), (j, n) detection of alpha-tubulin (green), (c, $, \mathbf{g}, \mathbf{k}, \mathbf{o})$ DAPI (blue), (d, h l, p) merge. Bars: $10 \mu \mathrm{m}(\mathbf{a}-\mathbf{h}), 20 \mu \mathrm{m}(\mathbf{i}-\mathbf{p})$.

m-p, arrows) demonstrated by alpha-tubulin decoration. Thus, depletion of $O d f 1$ neither affected the formation of the manchette nor the recruitment of CFAP52. Localization of CFAP52 to the manchette microtubules is therefore not mediated by ODF1.

Endogenous CFAP52 localizes to centrosome/basal body, intercellular bridge and spindle poles in somatic cells. We have proven prevalent low-level expression of Cfap52 by nested RT-PCR. In order to substantiate these findings, we performed immunoblotting using total proteins obtained from mouse tissues as well as from NIH3T3 cells. CFAP52, in the expected molecular mass of $\sim 68 \mathrm{kDa}$, was clearly detectable in all probes demonstrating once more that CFAP52 is not restricted to specific tissues (Fig 6). Our results are corroborated by Western blot validation of anti-CFAP52 (www.cusabio.com). Next, we used NIH3T3 cells 


\section{NIH3T3}

Testes

Brain

Liver

Kidney

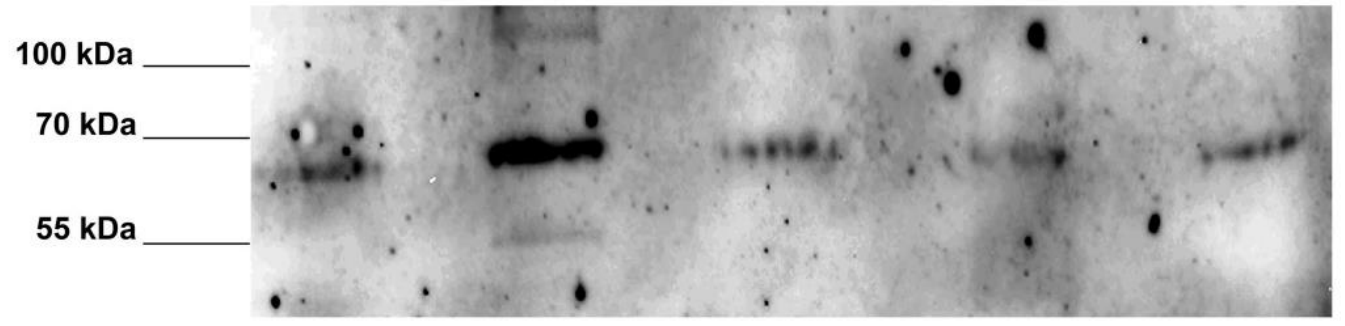

Figure 6. Detection of CFAP52 in mouse tissues. Total proteins of mouse tissues and of NIH3T3 cells were separated on a denaturing SDS-gel, transferred to Hybond ECL, and incubated with the anti-CFAP52 antibody. Chemiluminescence detection of the antibody. In all probes, CFAP52 with the predicted molecular mass of $68 \mathrm{kDa}$ was detected.

to isolate the coding sequence of Cfap52 and generated a fusion protein with EGFP by cloning Cfap52 in frame to the N-terminal end of Egfp in plasmid pEgfp-N1. The plasmid $p C f a p 52:: e g f p$ was transfected into NIH3T3 cells, and its distribution analyzed. Besides a more or less uniform background fluorescence we found a specific concentration of the CFAP52::EGFP fusion protein in two closely associated spots in the vicinity of the nucleus. Immuno-decoration with anti- $\gamma$-tubulin antibodies as a centrosomal marker revealed that CFAP52::EGFP colocalized with the centrosome in NIH3T3 cells (Fig. 7A a-d). We then asked whether the centrosomal location is caused by overexpression of the fusion protein, or otherwise is a feature of the endogenous protein characterizing CFAP52 as a novel centrosomal protein. To this end, NIH3T3 cells were treated for immuno-decoration and double stained with anti-CFAP52, and either anti- $\gamma$-tubulin or anti-acetylated tubulin antibodies (Fig. 7B). We detected the endogenous CFAP52 protein in the centrosome by colocalization with the centrosomal marker protein $\gamma$-tubulin (Fig. 7B a-d). Furthermore, CFAP52 is found in the cytoplasmic bridge linking the two daughter cells (Fig. 7B e-h). In this case, the cytoplasmic bridge is highlighted by acetylated tubulin. CFAP52 did not colocalize with acetylated tubulin but instead seemed to be concentrated at the tip of the cytoplasmic bridge near the plasma membrane. NIH3T3 cells generate non-motile, solitary cilia known as primary cilia that are near the plasma membrane. NIH3T3 cells generate non-motile, solitary cilia known as primary cilia that are
also easily identifiable by immuno-decoration for acetylated tubulin. The primary cilium as well as its associated daughter centriole at its base were identified by acetylated tubulin (Fig. 7B i-l). CFAP52 was not found in the ciliary axoneme but is highly concentrated at the basal body and at the daughter centriole. Additionally, CFAP52 is concentrated at the spindle poles that are highlighted by acetylated tubulin staining when captured at low intensity (Fig. 7B m-p). Omitting the anti-CFAP52 antibody and using both secondary antibodies for incubation in the controls demonstrated decoration of the centrosome with anti-acetylated tubulin antibodies (in red) but did not show any green fluorescence (Fig. $7 \mathrm{~B} \mathrm{q}-\mathrm{t}$ ).

Centrosomal recruitment of CFAP52 isoforms. We have identified CFAP52 as a novel centrosomal protein in NIH3T3 cells. CFAP52 is encoded by the Wdr16/Cfap52 gene on chromosome 11 (11B3) in mice. The gene consists of 14 exons, and encodes three isoforms by alternative splicing. The longest isoform (NP_082239.2) consists of 620 amino acids (aa) encoded by exons 1-14. The isoform denominated X1 (XP 006534328.1) consists of 577 aa by skipping of exon 4. Isoform 203 (Q5F201) has a postulated length of 342 aa and is encoded by exons $1-8$. We cloned the isoforms from NIH3T3 cells and generated in fusion plasmids to Egfp. Additionally, we generated another fusion protein named isoform X1-Nterm that consists of 309 aa of CFAP52 by skipping exons 4 and all C-terminal exons 9-14 (Fig. 8). According to ProSite (https://prosite.expasy.org) the full-length sequences CFAP52 and CFAP52-X1 contain two WD-repeats regions comprising aa 60-195, and 328-620 (related to the full-length isoform), and five WD-repeats-2. Referring to the full-length isoform, the WD-repeats-2 sequences comprise aa 107-142, 413-446, 457-490, 541-582, and 583-620. The isoform 203 contains one WD-repeat region and one WD-repeat-2, comprising aa 107-142. The WD-region of the isoform X1-Nterm differs from that of isoform 203 by a deletion of 43 aa (Fig. 8). To investigate the impact of the WDdomains for centrosomal recruitment of CFAP52 the egfp-fusion plasmids were transfected into NIH3T3 cells and the fusion proteins detected by their green auto-fluorescence (Fig. 9). The centrosome was decorated by immunostaining for their marker proteins either $\gamma$-tubulin or pericentrin. As observed previously for the fulllength CFAP52::EGFP fusion protein (Fig. 7A) both, the EGFP-fusion proteins of isoform 203 as well as of the $\mathrm{N}$-terminal end of isoform X1 (X1-Nterm), are concentrated in the centrosome (Fig. 9).Taken together, our data indicate that (1) the C-terminal region comprising the second WD-repeats domain is not essential for centrosomal recruitment and that (2) parts of the first WD-repeats domain encoded by exon 4 are also not essential for centrosomal recruitment. Thus, a centrosomal targeting sequence, if at all present, must reside in the $\mathrm{N}$-terminal end of CFAP52, represented by isoform X1-Nterm, or most likely the WD-repeats domains taken as a whole are responsible to direct CFAP52 to the centrosome. 
A

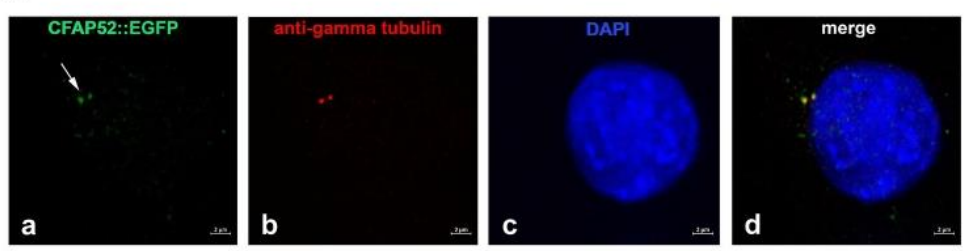

B
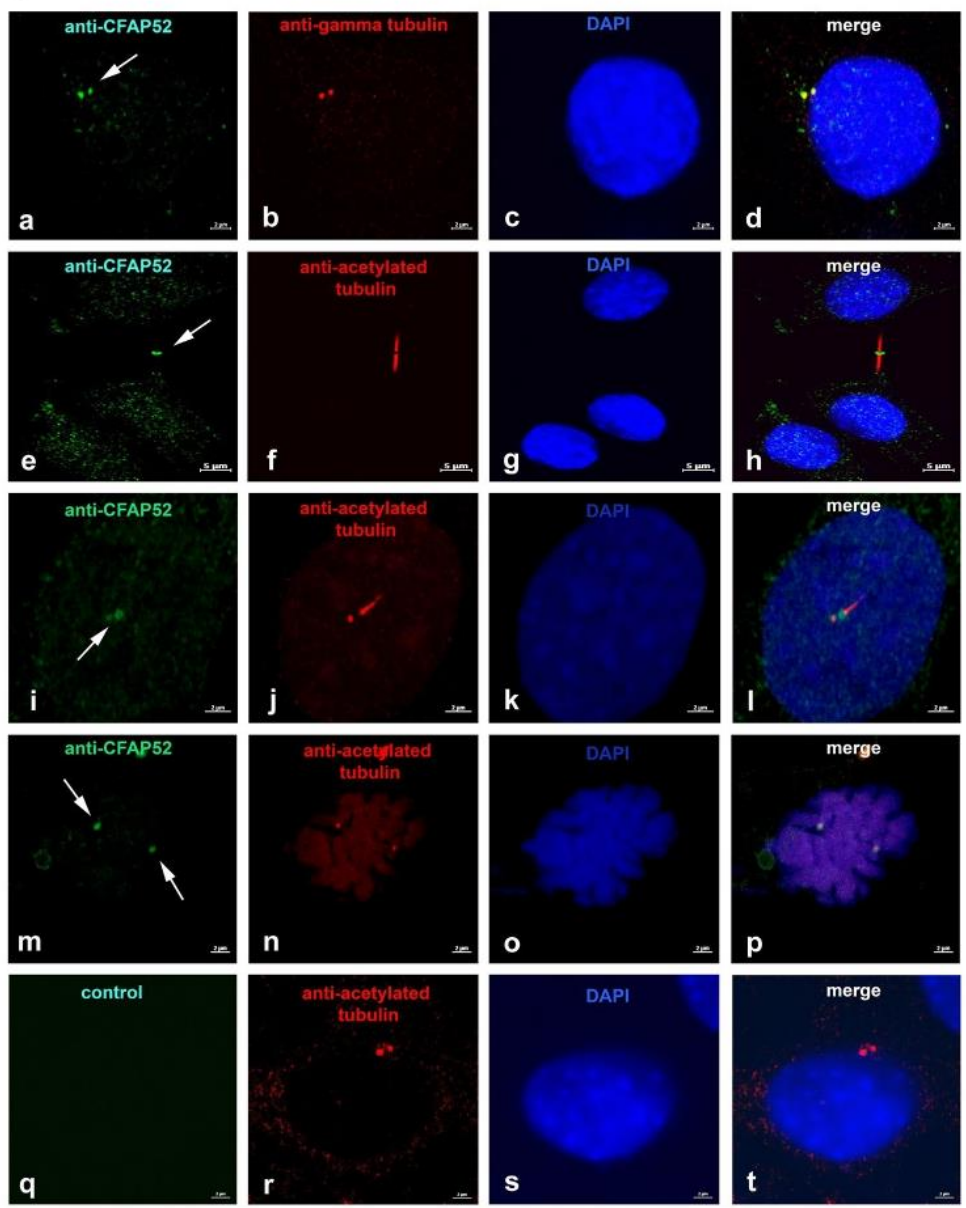

Figure 7. CFAP52 locates to the centrosome, the basal body, the intercellular bridge, and the spindle poles in NIH3T3 cells. (A) The full-length $p$ Cfap 52..Egfp expression plasmid was transfected into NIH3T3 cells and detected by auto-fluorescence (green) (a). Immuno-decoration of the centrosome with the centrosomal marker $\gamma$-tubulin (red) revealed localization of (green) (a). Immuno-decoration of the centrosome with the centrosomal marker $\gamma$-tubulin (red) revealed localization of
CFAP52 in the centrosome (b-d). Bars are of $2 \mu \mathrm{m}$. (B) Endogenous expression of CFAP52 in NIH3T3 cells was detected by double immunostaining with anti-CFAP52 (green) and either anti- $\gamma$-tubulin (red) or anti-acetylated tubulin (red). $\gamma$-tubulin and acetylated tubulin decoration for detection of the centrosome, acetylated tubulin for decoration of the basal body, the spindle poles, the intercellular bridge, and the primary cilium. The endogenous CFAP52 (arrows) locates to the centrosome (a-d), the intercellular bridge (e-h), the basal body and its associated daughter centriole (i- $\mathbf{l}$ ), and the spindle poles ( $\mathbf{m}-\mathbf{p}$ ). Omitting anti-CFAP52 showeddecration of the centrosome by anti-actylated tubulin staining (red) but no false CFAP52 staining (q-t). Nuclear counterstain with DAPI (blue). Bars are of $2 \mu \mathrm{m}$ except for $(\mathbf{e}-\mathbf{h})$, which are of $5 \mu \mathrm{m}$. 
CFAP52 (NP_082239.2) CFAP52-X1 (XP_006534328.1) CFAP 52-203 (Q5F201) CFAP 52-X1-Nterm

CFAP52 (NP_082239.2) CFAP 52-X1 (XP_006534328.1) CFAP 52-203 (Q5F201) CFAP 52-X-Nterm

CFAP52 (NP_082239.2) CFAP52-X1 (XP 006534328.1) CFAP $52-X 11 \times 12$ CFAP52-203 (Q5F201)

CFAP52 (NP_082239.2) CFAP 52-X1 XP_006534328.1 CFAP52-203 (Q5 $\bar{F} 201$ CFAP52-X-Nterm

CFAP52 (NP_082239.2 CEAP52-X1 (XP_006534328.1) CFAP 52-203 (Q5F201) CFAP52-X1-Nterm

CFAP52 (NP_082239.2) CEAP 52-X1 (XP_006534328.1) CFAP 52-203 (05F201) CFAP52-X1-Nterm

CFAP52 (NP_082239.2) CFAP52-X1 (XP_006534328.1) CFAP52-203 (Q5F201) CFAP52-X1-Nterm

CFAP52 (NP_082239.2) CFAP 52-X1)(XP_006534328.1 CFAP 52-203 ( $0 \overline{5}$ F201 CFAP52-X1-Nterm

CFAP52 (NP_082239.2 CFAP 52-X1 (XP_006534328.1) CFAP 52-203 (Q5F201) CFAP 52-X1-Nterm

CFAP52 (NP_082239.2) IsoformX1 (XP_006534328.1) CFAP 52-203 $(05 \overline{5} 201)$ CFAP52-X1-Nterm

CFAP52 (NP_082239.2) CFAP 52-X1 (XP 006534328.1) CFAP52-X1 (XP 00653 CFAP52-X1-Nterm
MEEQVLPELDVAELELQAVIGFNGHVPNGLRCHPDQEHLIYPLGCTVLIQAINTNEQNF| 60 MEEQVLPELDVAELELQAVIGFNGHVPNGLRCHPDOEHLIYPLGCTVLIOAINTNEQNAL 60 MEEQVLPELDVAELELOAVIGENGHVPNGLRCHPDOEHLIYPLGCTVLIOAINTNEONHI 60 MEEQVLPELDVAELELOAVI GFNGHVPNGLRCHPDOEHLIYPLGCTVLIOAINTNEONA I 60 年

HGHGNNVSCVTISREGDYIASGOVTFMGFRADI ILWDFRRREL IARLSLHKGKIEALAFS 120 HGHGNVVSCVTISREGDYTASGOVTEMGERADIILODERZRELIARISLHKGKIFALAFS 20 H HGHGNNVSCVTISREGDYIASGQVTFMGFRADIILWDFRKRELIARLSLLKKGKIRALAFS 12

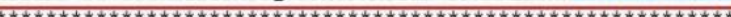

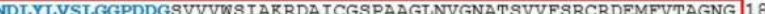

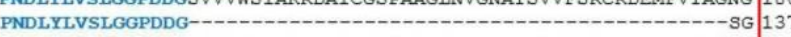
PNDLYLVSLGGPDDGSVVVWSIARRDAICGSPAAGINVGNATSVVFSRCRDEMFVTAGNG 180 $\left.\begin{array}{l}\text { PNDLYLVSLGGPDDGSVVVWSIARRDAICGSPAAGLNVGNATSVVFSRCRDEMFVTAGNG } \\ \text { PNDLYLVSLGGPDG }\end{array}\right]$

TIRVWELDLPNRKIWPTECOTGQMRRIVLSTGMADDDSFFYLGTTTGDILRMNPRTRLLA 240 TIRVWELDLPNRRIW TECOTGOMRRIVLSTGMADDDSFFYIGTMTGDILRMNPRTRLLA 197 TIRVWELDLPNRRIWTECOTGQMRRIVLSTGMADDDSFFYLGTTTGDILRMNPRTRLLA 240 TIRVWELDLPNRR IWTECQTGQMRRIVLSTGMADDDSFFYLGTTTGDILRMNPRTRLIA 19

DTGPVRDRFSLGVSALRCLRMGGLIVGSGAGLLIFCRSPSYRPIRRVQIQGGITSITLRG 300 DTGPVRDRFSLGVSALRCLRMGGLLVGSGAGLIIFCRSPSYKPIRRVQLQGGITSITLRG 257 DTGPVRDRFSLGVSALRCLRMGGLIVGSGAGLLIFCRSPSYRPIRRVQLQGGITSITLRG 300 DTGPVKDKFSLGVSALRCLRMGGLLVGSGAGLLIFCRSPSYKPIKRVQLOGGITSITTLRG 257

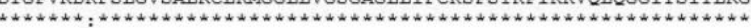

EGHQFFVGTEESHIYRVNFTDFKETLI ATCHFEAVQDIVFPFGTAELFATCARRDIRVWH 360 EGHOFFVGTEESHIYRVNETDFRETLIATCHFEAVQDIVFPFGTAELFATCARKDIRVWH 317 EGHOFFVGTEESHIYRVNFTDFRETLIATCHFEAVQDIVFPL--------------- 342

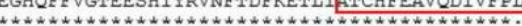

TMSRRELLRITVPNMTCHGIDFMRDGRSI I SAWDDGR IRAFAPESGRLMYTINSAHRIGV 42 TMSRRELIRITVPNMTCHGIDFMRDGRSIISAWDDGRIRAFAPESGR RMYTINSAHRIGV 37 -

TAIATTSDCKRI I SGGGEGEVRVWQVGCQTORLEEALREHKSSVSCIRVKKNNEECVTAS 48 TAIATTSDCKRI I SGGGEGEVRVWQVGCQTORLEEALKEHKSSVSCIRVKKNNEECVTAS 43

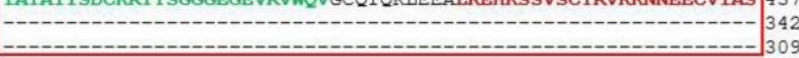

TDGTCI IWDLVRLRRNQMI LANTL FQCVCYHPEEFQI ITSGTDRRIAYWEVFDGSVIREL 540 TDGTCI IWDLVRLRRNOMILANTLFQCVCYHPEEFQIITSGTDRRIAYWEVFDGSVIREI 497

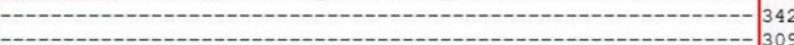

BGSLSGS INGMD I TQEGGHFVTGGHDHLVKVWD YNEGEVTHVGVGHSGN IMAMRI SPGNO 600

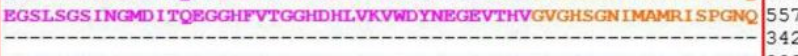

YIVSYSADGAILRWKYPFAS

YIVSVSADGAILRWKYPFAS 57

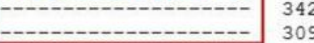

Figure 8. CFAP52 isoforms. Wdr16/Cfap52 encodes three isoforms by alternative splicing. The full-length isoform CFAP52 (NP 082239.2) consists of 620 amino acids. The isoform denominated CFAP52-X1 (XP_006534328.1) consists of 577 amino acids, and the isoform CFAP52-203 (Q5F201) has a postulated length of 342 amino acids. The sequence denominated CFAP52-X1-Nterm is the N-terminal end of isoform X1 consisting of 309 aa and was artificially cloned. The WD40 repeats regions (according to Prosite circula profile) comprise aa 60 to 195 and 328 to 620 (enframed in red). The WD40 repeats (according to Prosite WD-repeats-2) are colour-coded. 

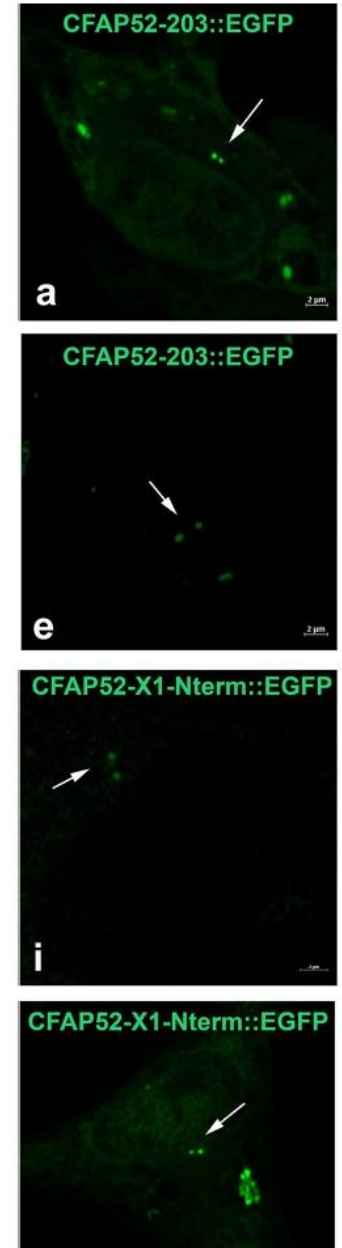

m
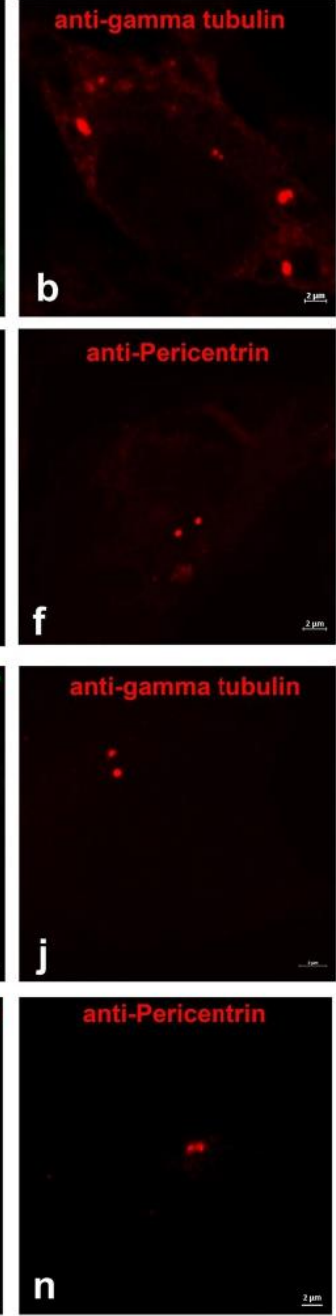
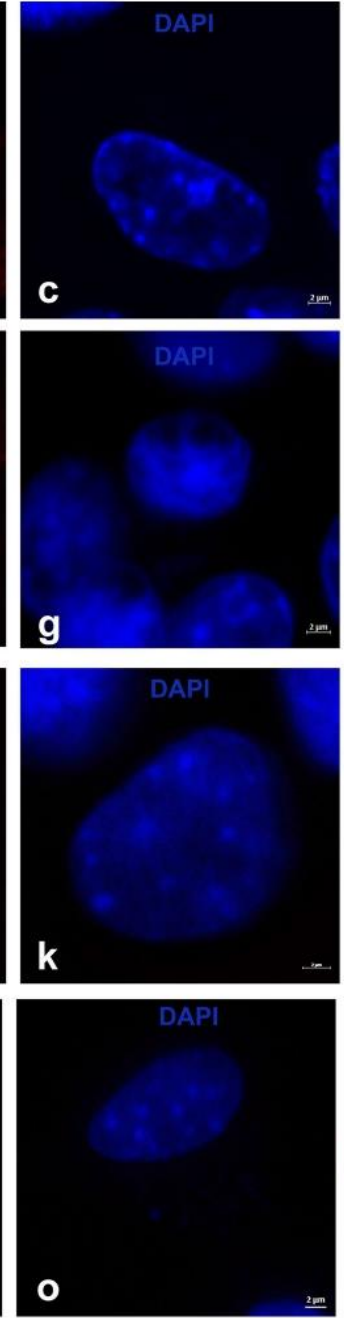

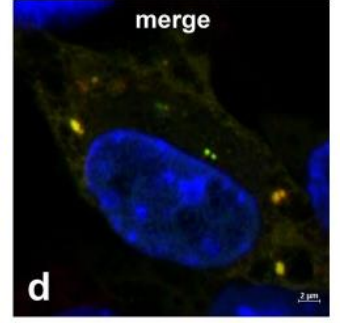

merge
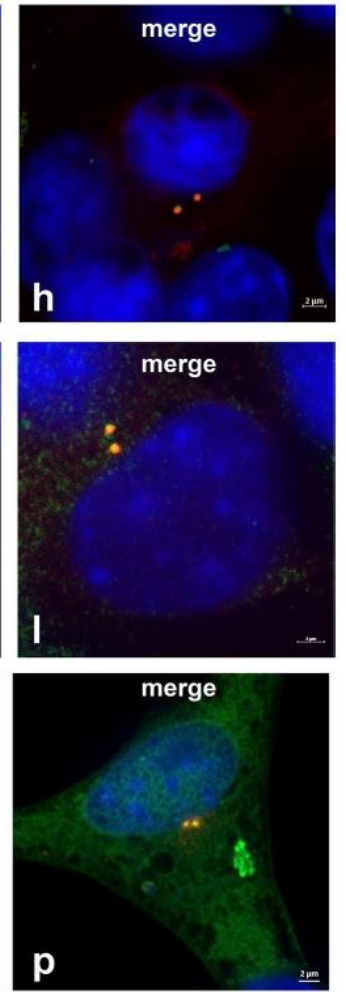

Figure 9. Centrosomal location of EGFP-fusion proteins of CFAP52 isoforms 203 (CFAP52-203::EGFP) and the N-terminal end of isoform X1 (CFAP52-X1-Nterm::EGFP). Expression plasmids for the isoform 203, $p C f a p 52-203:: E g f p$ (a-h), and the N-terminal sequence of CFAP52-isoform-X1, pCfap52-X1-Nterm::Egfp (i-p) were transfected into NIH3T3 cells and detected by the EGFP auto-fluorescence (green, a, e, i, m). Decoration of the centrosomes by immunostaining for the centrosomal marker proteins either $\gamma$-tubulin (anti-gammatubulin, $\mathbf{b}, \mathbf{j}$ ) or Pericentrin (anti-Pericentrin, $\mathbf{f}, \mathbf{n}$ ) (both in red). Nuclear counterstain with DAPI (blue). Arrows pointing to the centrosomal location of the fusion proteins. Bars are of $2 \mu \mathrm{m}(\mathbf{a}-\mathbf{p})$

\section{Discussion}

WDR16/CFAP52 is a member of the WD40-protein family characterized by the WD-repeat domain. The WD40repeats, also known as GH-WD repeats, are repetitive motifs consisting of a conserved core bracketed by GH (Gly-His) and WD, and additional sequences of variable lengths ${ }^{1,38}$.

The WD40-domains were first described in $\beta$ subunits of transducin and other $\mathrm{G}$ proteins isolated from bovine tissues, and in the SCF (SKP1-CUL1-F-box protein) ubiquitin ligase complex component CDC4. WDrepeat proteins fulfill diverse biological functions including RNA synthesis/processing, signal transduction, cytoskeleton assembly, mitotic spindle formation, vesicular trafficking and cell growth ${ }^{6,39}$. WD-repeat domains are hence widespread and highly conserved in eukaryotes. Particularly, the WD40-repeat protein CFAP52/ WDR16/WDRPUH was initially identified and found to be upregulated in human hepatocellular carcinomas. 
Its overexpression in NIH3T3 cells accelerated cell growth, whereas inhibition of WDRPUH/CFAP52 reduced the growth of human liver carcinoma cells and induced apoptosis ${ }^{3,7}$

Previous investigation of Cfap52 indicated that its expression correlated with the presence of motile cilia ${ }^{7}$. Furthermore, WDR16/CFAP52 was annotated in the Ciliome Database and was identified in mouse ciliated tissues $^{40}$. Moreover, the Wdr16 gene was identified as FOXJ effector gene altogether indicating a correlation of WDR16/CFAP52 with ciliation especially with the generation of motile cilia ${ }^{41}$. Proteins containing specific domains, especially coiled-coil domains, WD40-repeat domains, or tetratricopeptide repeat domains have all been linked to cilia $^{40,42,43}$. In this regard, the intraflagellar transport component IFTA-1 is a WD repeat-containing protein $^{44}$. However, WDR16/CFAP52 is not annotated in Syscilia Gold Standard (https://www.syscilia.org/golds tandard.shtml) and therefore seems not to be an essential ciliary component.

The reported association of the cilia and flagella associated protein 52 (CFAP52/WDR16) with ciliation and its high expression in testis offered the unique opportunity to investigate its sub-cellular distribution in more detail. The formation of the flagellum starts in haploid spermatids and is well known step-by-step from the migration of the paired centrioles towards the caudal end of the nucleus, their insertion into the implantation fossa, and their progressive transformation into the basal body, the connecting piece, and eventually the outgrowth of the flagellum. The proximal centriole inserts into the implantation fossa and mediates the proper linkage between head and tail by the head-to-tail coupling apparatus (HTCA). The distal centriole, on the other hand, gives rise to the basal body that in turn will seed flagellum formation ${ }^{10,45}$. Furthermore, flagellum and connecting piece are both discernable at light-microscopic resolution and are easily identifiable using immuno-decoration.

Formation of HTCA and sperm tail requires many proteins that are presumably transported by a transient microtubular structure, the manchette $e^{24,27}$. The HTCA is essential for functional sperm, and its malformation causes acephalic spermatozoa and male infertility due to fragile attachment of sperm head and tail or even detachment ${ }^{12,46}$. Albeit essential for fertilisation, the knowledge of the molecular composition of the HTCA is just in the beginning. We therefore asked, whether CFAP52 as an annotated ciliary component is associated with the flagellum or its development. We found that, opposed to previous reports, CFAP52 locates to the transient manchette in round and elongating spermatids. CFAP52 might thus be involved in the stabilization of the structure or in supporting delivery of cargos, or is a cargo protein itself. Later on, CFAP52 was found in the sperm tail with the strongest staining in the principal piece. A weaker decoration was observed in the middle piece that might be explained by a reduced antibody accessibility due to the fact that mitochondria and outer dense fibres encapsulate the central region of the axoneme. Although, location of CFAP52 to manchette microtubules and sperm tail resembles that of the major outer dense fibre protein ODF1, recruitment of CFAP52 to these structures is independent of ODF1.

Expression analyses of Cfap52 by RT-PCR, notably nested RT-PCR, revealed transcription in all tissues investigated including those that do not harbour motile cilia, e.g. ovary. Additionally, Cfap52 was also expressed in the fibroblast cell line NIH3T3. Cfap52 cDNAs were isolated from NIH3T3 cells and cloned in frame to egfp to generate EGFP-fusion proteins when ectopically expressed. Investigations of the subcellular distributions of CFAP52::EGFP-fusion proteins as well as of the endogenous CFAP52 protein demonstrated location of CFAP52 in the centrosome, the mitotic spindle poles, the tip of the mid-bodies, and at the base of primary cilia. However, the primary cilium axoneme was not decorated by CFAP52. We observed centrosomal location of full-length CFAP52 and its isoforms including its N-terminal part comprising a single truncated WD40-domain. We propose that centrosomal recruitment most likely is mediated by the WD40-domain that generally functions in mediating protein-protein interactions ${ }^{6}$

Our data indicate, that CFAP52 associates with a subset of microtubular structures, especially centrosome/ centriole derived structures. As WD-repeat proteins mediate protein interactions, CFAP52 might likewise act in assembly or stabilization of protein complexes constituting the centrosomal/basal body matrix and the sperm tail $^{6}$. Furthermore, the location of CFAP52 to the sperm tail but not to the axoneme of primary cilia suggests that CFAP52 fulfils specific functions related to the stability, maintenance, or motility of the sperm tail. A loss-offunction of CFAP52 might therefore primarily affect the sperm tail and in turn male fertility. It is currently not known whether mutations in CFAP52 affect male fertility although mutations in WD-repeat proteins, as e.g. in WDR66/CFAP251, WDR96/CFAP43, and WDR52/CFAP54, causing human male infertility due to the sperm flagella defects have been reported ${ }^{47-49}$. Knock down or mutation studies of CFAP52 in zebrafish and humans, respectively, indicated impaired motile cilia in the ependym causing hydrocephalus, and impaired motile, solitary cilia in the node causing left-right symmetry disorders ${ }^{7,8}$. However, it has not been investigated whether primary cilia and the sperm tail are also affected, causing ciliopathies and male infertility due to sperm motility disorders, respectively. We anticipate our results to be a starting point for a more sophisticated analysis of the role CFAP52 plays in the formation of centrosome-derived structures and in male germ cells using knock out approaches.

Materials and methods

cDNA synthesis and RT-PCR. Total RNA from adult mouse tissues (testis, liver, brain, ovary, kidney, spleen, epididymides) as well as from NIH3T3 mouse fibroblasts was prepared using peqGOLD RNApure ${ }^{\mathrm{m}}$ (PeqLab, Erlangen, Germany) following the recommendations of the manufacturer. Total RNA was digested with Ambion ${ }^{*}$ TURBO DNA-free ${ }^{\mathrm{m}}$ DNase (Life Technologies). cDNA was synthesized using Maxima First Strand cDNA Synthesis (ThermoFisher Scientific) ${ }^{31}$. Detection of transcribed sequences was performed by RTPCR using the following primer pairs: CFAP52-NheI-X1-For (GCTAGCATGGAAGAACAAGTTTTACC) and CFAP52-X1-BamHI-R (GGATCCGAAGCAAATGGGTATTTC) generating a product of $1871 \mathrm{bp}$. After first PCR, the following primers were used to perform a nested PCR: CFAP52-For (CCAGCGTGGTCTTCTCTA GG) and CFAP52-Rev (CCTTCACAGGCCCAGTATC) generating a fragment of $255 \mathrm{bp}$. Gapdh was amplified 
using Gapdh-For (GTATGACTCCACTCACGGCA) and Gapdh-Rev (GTCAGATCCACGACGGACAC) generating a fragment of $594 \mathrm{bp}$. PCR products were sequenced.

Plasmid constructs. Four different $C f a p 52$ coding sequences were amplified by PCR. Cfap52 full-length (NP_082239.2) and Cfap52 is oform X1 (XP_006534328.1) were amplified using the primer pair CFAP52-NheIX1-For (5'-GCTAGCATGGAAGAACAAGTTTTACC-3') and CT-CFAP52-X1-BamHI-R (5'-GGATCCGAA GCAAATGGGTATTTC-3'). The isoform Cfap52-203 (Q5F201) and the N-terminal region of the isoform X1 were amplified using the following primer pair: CFAP52-NheI-For (5'-GCTAGCCATGGAAGAACAAGTTTT AC-3') and CFAP52-KpnI-203-Rev (5'-GGTACCAATGGAAAGACAATGTCCTGG -3'). PCR products were cloned into pJET1.2/blunt (ThermoFisher Scientific) followed by NheI/BamHI or NheI/KpnI digestion and subcloning into $p E G F P-N 1$ (Clontech Lab., \#U55762). Correct reading frames were confirmed by sequencing.

Cell culture and immunocytochemistry. NIH3T3 cells (ATCC CRL-1658) were maintained in Dulbecco's Modified Eagle's Medium (DMEM), 10\% (v/v) fetal bovine serum (FBS), $1000 \mathrm{U} / \mathrm{ml}$ penicillin, $1000 \mu \mathrm{g} /$ $\mathrm{ml}$ streptomycin, and $20 \mathrm{mM}$ L-Glutamine (all Gibco) at $37^{\circ} \mathrm{C}$ and $5 \% \mathrm{CO}_{2}$ NIH3 3 cells were grown on glass coverslips in 6-well plates and the plasmid DNA was transfected using EndoFectin ${ }^{m t}$ Max Transfection Reagent, using the manufacturer's instructions (GeneCopoeia). Twenty-four hours post transfection, cells were rinsed with phosphate-buffered saline (PBS) and fixed either in methanol for $10 \mathrm{~min}$ at $-20^{\circ} \mathrm{C}$ or in $3.7 \%$ paraformaldehyde (PFA) for $20 \mathrm{~min}$ at $4{ }^{\circ} \mathrm{C}$. Samples were then permeabilized with $0.3 \%$ Triton X-100 in PBS for $10 \mathrm{~min}$ at room temperature. Cells were rinsed in PBS, and non-specific binding sites were blocked by incubation in PBS containing $1 \%$ BSA and $0.3 \%$ Triton X-100 for $1 \mathrm{~h}^{31}$. Samples were incubated with primary antibodies: anti-CFAP52 (CSB-PA839781LA01HU; Wuhan Huamei Biotech Co., Ltd., Wuhan, China, Cusabio), antiPericentrin (PRB432C, Covance), anti-acetylated tubulin (6-11B-1; Sigma-Aldrich), anti- $\gamma$ tubulin (GTU-88, Sigma-Aldrich) at $37^{\circ} \mathrm{C}$ for $1 \mathrm{~h}$. Secondary antibodies used are goat anti-mouse-IgG DyLight 488 (\#35503, ThermoScientific), and goat anti-rabbit-MFP590 (\#MFP-A1037, Mobitec). DNA was counterstained with DAPI. Images were taken by confocal microscopy (LSM 780, Zeiss) and processed using Adobe Photoshop 7.0.

Immunocytology on testicular cell suspensions. Fresh testes from laboratory mice of strain C57/Bl6 were minced in PBS containing $2 \%$ paraformaldehyde, $0.02 \%$ SDS, and $0.15 \%$ Triton X-100, and transferred onto superfrost slides. Cells were blocked for $1 \mathrm{~h}$ in PBS containing $1 \%$ BSA and $0.3 \%$ Triton X-100. Anti-CFAP52 (CSB-PA839781LA01HU; Wuhan Huamei Biotech Co., Ltd., Wuhan, China, Cusabio) was used as 1:100 dilution and incubated at $4{ }^{\circ} \mathrm{C}$ overnight. CFAP52 was detected using the secondary antibody goat anti-rabbit-IgG $(\mathrm{H}+\mathrm{L})$ Alexa Fluor R 555 (F[ab]2 fragment; \#A21430, Life Technologies) used as 1:1,000 dilution. Additionally, the following first antibodies were used: anti- $\alpha$-tubulin (DM1A, Calbiochem, \#CP06), anti- $\gamma$-tubulin (GTU-88, Sigma-Aldrich, \#T6557), anti-EB3 (EB3(7), Santa Cruz, sc-136405), anti-SUN3 and anti-SUN4 ${ }^{36,50}$, and detected using goat anti-mouse-IgG DyLight488 (Thermo Scientific, \#35503) or goat anti-guinea pig-IgG Alexa Fluo 488 (Life Technologies, \#A11073). DNA was counterstained with DAPI (4',6-Diamidino-2-phenylindole; Sigma D-9542), and the acrosome was decorated with FITC-labelled peanut lectin (PL-FITC). Images were taken by confocal microscopy (LSM 510, Zeiss) and processed using Adobe Photoshop 7.0.

Histology. Testes were fixed in $4 \%$ paraformaldehyde in phosphate-buffered saline (145 mM NaCl, $7 \mathrm{mM}$ $\mathrm{Na}_{2} \mathrm{HPO}_{4}$, and $3 \mathrm{mM} \mathrm{NaH}_{2} \mathrm{PO}_{4}$; $\mathrm{PBS}$ ) and embedded in paraffin. $4 \mu \mathrm{m}$ sections were cut and placed onto Superfrost slides. After deparaffinization and rehydration probes were treated for enzymatic epitope retrieval by incubation with $0.5 \%$ Trypsin for $10-20 \mathrm{~min}$ at $37^{\circ} \mathrm{C}$. Specimen were blocked in PBS containing $1 \%$ BSA and $0.3 \%$ Triton X-100 followed by antibody incubation with rabbit anti-CFAP52 (CSB-PA839781LA01HU) at $4{ }^{\circ} \mathrm{C}$ overnight. Anti-CFAP52 was detected with goat anti-rabbit-MFP590 (\#MFP-A1037, Mobitec) (diluted 1:100). The acrosome was stained with FITC-labelled peanut-lectin (PL-FITC) and the DNA by DAPI (4', 6-Diamidino2-phenylindole; Sigma D-9542).

Western blotting. Proteins were prepared from mice tissues (testes, brain, liver, kidney) and NIH3T3 cells. Tissues were homogenized with a Dounce homogenizer in lysis buffer [ $150 \mathrm{mM} \mathrm{NaCl}, 1 \%$ Nonidet P40,0.5\% sodium deoxycholate, $0.1 \%$ SDS, $50 \mathrm{mM}$ Tris- $\mathrm{HCl}, \mathrm{pH}$ 7.6, protease inhibitor cocktail (Halt Protease Inhibitor Cocktail 100X, Thermo Fisher Scientific, \#78438)]. NIH3T3 cells were harvested by trypsinization followed by rinsing twice in phosphate-buffered saline (PBS). NIH3T3 cell pellet was resuspended in lysis buffer. Protein lysates were sonicated 3 times for $45 \mathrm{~s}$ each. Total protein lysates were resuspended in $2 \times \mathrm{SDS}$-sample buffer and heated to $95^{\circ} \mathrm{C}$ for $10 \mathrm{~min}$. Proteins were separated on SDS-PAGE and transferred onto nitrocellulose membrane (Amersham Hybond-ECL, GE Healthcare) ${ }^{51,52}$. The membrane was blocked in $5 \%$ dry milk in TBST (10 mM Tris- $\mathrm{HCl} \mathrm{pH} \mathrm{7.6,150} \mathrm{mM} \mathrm{NaCl,} \mathrm{0.05 \%} \mathrm{Tween} \mathrm{20)} \mathrm{for} \mathrm{one} \mathrm{hour.} \mathrm{Afterwards,} \mathrm{the} \mathrm{membrane} \mathrm{was} \mathrm{incubated} \mathrm{with}$ the primary antibody rabbit anti-CFAP52 (CSB-PA839781LA01HU) in blocking solution at $4{ }^{\circ} \mathrm{C}$ overnight. First antibodies were detected by incubation with HRP-conjugated secondary antibody goat anti-rabbit IgG (Jackson ImmunoResearch, WestGrove, PA, USA). Signals were detected using ClarityMax Western ECL Substrate (BioRad, \#1705062) and images were captured with Chemdoc (Bio-Rad).

Ethics statement. All mouse experiments were reviewed and approved by the animal welfare commission of the University Medical Faculty and Niedersächsisches Landesamt für Verbraucherschutz und Lebensmittelsicherheit. Licence for animal experiments has been obtained by the Institute of Human Genetics. The guidelines 
of the German Animal Welfare Act (German Ministry of Agriculture, Health and Economic Cooperation) were strictly followed in all aspects of mouse work. Odf1-ko mice has been previously described ${ }^{28}$.

Data availability

All data generated or analysed during this study are included in this published article.

Received: 6 May 2020; Accepted: 7 August 2020

Published online: 28 August 2020

References

1. Neer, E. J., Schmidt, C. J., Nambudripad, R. \& Smith, T. F. The ancient regulatory-protein family of WD-repeat proteins. Nature 371, 297-300. https://doi.org/ 10.1038/371297a0 (1994).

2. Smith, T. F., Gaitatzes, C., Saxena, K. \& Neer, E. J. The WD repeat: a common architecture for diverse functions. Trends Biochem. Sci. 24, 181-185. https://doi.org/10.1016/s0968-0004(99)01384-5 (1999).

3. Silva, F. P., Hamamoto, R., Nakamura, Y. \& Furukawa, Y. WDRPUH, A Novel WD-repeat-containing protein, is highly expressed in human hepatocellular carcinoma and involved in cell proliferation. Neoplasia $7,348-355$. https: //doi.org/10.1593/neo.04544 (2005)

4. Smith, T. F. Diversity of WD-repeat proteins. Subcell. Biochem. 48, 20-30 (2008).

5. Atlas of Genetics and Cytogenetics in Oncology and Haematology https://atlasgeneticsoncology.org/Genes/GC_CFAP52.html

6. Li, D. \& Roberts, R. Human genome and diseases: review WD-repeat proteins: structure characteristics, biological function, and . Li, D. \& Roberts, R. Human genome and diseases: review WD-repeat proteins: structure characteristics, biological function,
their involvement in human diseases. CMLS Cell. Mol. Life Sci. 58, 2085-2097. https://doi.org/10.1007/PL 00000838 (2001).

Hirschner, W. et al. Biosynthesis of Wdr16, a marker protein for kinocilia-bearing cells, starts at the time of kinocilia formaHirschner, W. et al. Biosynt in rat, and wdr16 gene knockdown causes hydrocephalus in zebrafish. J. Neurochem. 101, 274-288. https://doi.org/10.111
tion tion in rat, and wdr16 gene knock $1 / \mathrm{j} .1471-4159.2007 .04500 . \times$ (2007)

8. Ta-Shma, A. et al. A human laterality disorder associated with a homozygous WDR16 deletion. Eur. J. Hum. Genet. 23, 1262-1265 (2015)

9. Owa, M. et al. Inner lumen proteins stabilize doublet microtubules in cilia and flagella. Nat. Commun. 10, 1143. https://doi. org/10.1038/ejhg.2014.265(2019).

10. Fawcett, D. W. The mammalian spermatozoon. Dev. Biol. 44, 394-436. https://doi.org/10.1016/0012-1606(75)90411-x (1975).

1. Russell, L. D., Russell, J. A., MacGregor, G. R. \& Meistrich, M. L. Linkage of manchette microtubules to the nuclear envelope and observations of the role of the manchette in nuclear shaping during spermiogenesis in rodents. Am. J. Anat. 192, 97-120. https:// doi.org/10.1002/aja.1001920202(1991)

12. Chemes, H. E. Sperm centrioles and their dual role in flagellogenesis and cell cycle of the zygote. Centrosome https://doi.

org/10.1007/978-1-62703-035-9_2 (2012).
3. Chemes, H. E. \& Rawe, V. Y. The making of abnormal spermatozoa: cellular and molecular mechanisms underlying pathological spermiogenesis. Cell Tissue Res. 341, 349-357. https://doi.org/10.1007/s00441-010-1007-3 (2010).

4. Hoyer-Fender, S. Centrosomes in fertilization, early embryonic development, stem cell division, and cancer. Atlas Genet. Cytogen. Oncol. Haematol. 16, 306-319. https://doi.org/10.4267/2042/47311 (2012)

15. Baltz, J. M., Oneeka Williams, P. \& Cone, R. A. Dense fibers protect mammalian sperm against damage. Biol. Reprod. 43, 485-491. https://doi.org/10.1095/biolreprod43.3.485 (1990).

6. Lindemann, C. B. Functional significance of the outer dense fibers of mammalian sperm examined by computer simulations with the geometric clutch model. Cell Motil. Cytoskelet. 34, 258-270. https://doi.org/10.1002/(SICI)1097-0169(1996)34:4\%3c258::AIDCM1\%3e3.0.CO;2-4 (1996)

17. Rattner, J. B. \& Olson, G. Observations on the fine structure of the nuclear ring of the mammalian spermatid. J. Ultrastruct. Res. 43, 438-444. https://doi.org/10.1016/S0022-5320(73)90020-8 (1973).

18. Kierszenbaum, A. L., Rivkin, E. \& Tres, L. L. Acroplaxome, an F-actin-keratin-containing plate, anchors the acrosome to the nucleus during shaping of the spermatid head. Mol. Biol. Cell 14, 4628-4640. https://doi.org/10.1091/mbc.e03-04-0226 (2003). 19. Rattner, J. B. \& Brinkley, B. R. Ultrastructure of mammalian spermiogenesis. J. Ultrastruct. Res. 41, 209-218. https://do $\mathrm{org} / 10.1016 / \mathrm{s} 0022-5320(72) 90065-2(1972)$

0. Kierszenbaum, A. L., Rivkin, E. \& Tres, L. L. Cytoskeletal track selection during cargo transport in spermatids is relevant to male fertility. Spermatogenesis 1, 221-230. https://doi.org/10.4161/spmg.1.3.18018 (2011).

21. Yan, W. Male infertility caused by spermiogenic defects: lessons from gene knockouts. Mol. Cell. Endocrinol. 306, 24-32. https:// doi.org/10.1016/j.mce.2009.03.003 (2009).

22. Chen, S. R. et al. The control of male fertility by spermatid-specific factors: Searching for contraceptive targets from spermatozoon's head to tail. Cell Death Dis. 7, e2472-e2472. https://doi.org/10.1038/cddis.2016.344 (2016)

23. Lehti, M. S. \& Sironen, A. Formation and function of the manchette and flagellum during spermatogenesis. Reproduction 151, R43-R54. https://doi.org/10.1530/REP-15-0310 (2016).

24. Cole, A., Meistrich, M. L., Cherry, L. M. \& Trostle-Weige, P. K. Nuclear and manchette development in spermatids of normal and azh/azh mutant mice. Biol. Reprod. 38, 385-401. https://doi.org/10.1095/biolreprod38.2.385 (1988).

5. Meistrich, M. L., Trostle-Weige, P. K. \& Russell, L. D. Abnormal manchette development in spermatids ofazh/azh mutant mice. Am. J. Anat. 188, 74-86. https://doi.org/10.1002/aja.1001880109 (1990)

26. Mendoza-Lujambio, I. et al. The Hook1 gene is non-functional in the abnormal spermatozoon head shape (azh) mutant mouse. Hum. Mol. 11, 1647-1658. https://doi.org/10.1093/hmg/11.14.1647 (2002).

27. Kierszenbaum, A. L. \& Tres, L. L. The acrosome-acroplaxome-manchette complex and the shaping of the spermatid head. Arch. Histol. Cytol. 67, 271-284. https://doi.org/10.1679/aohc.67.271 (2004).

28. Yang, K. et al. The small heat shock protein ODF1/HSPB10 is essential for tight linkage of sperm head to tail and male fertility in mice. Mol. Cell. Biol. 32, 216-225. https://doi.org/10.1128/MCB.06158-11 (2012).

29. Yang, K., Grzmil, P., Meinhardt, A. \& Hoyer-Fender, S. Haplo-deficiency of ODF 1/HSPB10 in mouse sperm causes relaxation of head-to-tail linkage. Reproduction 148, 499-506. https://doi.org/10.1530/REP-14-0370 (2014).

30. Shang, Y. et al. Essential role for SUN5 in anchoring sperm head to the tail. Elife 6, e28199. https://doi.org/10.7554/eLife.28199 (2017)

31. Tapia Contreras, C. \& Hoyer-Fender, S. CCDC42 localizes to manchette, HTCA and tail and interacts with ODF1 and ODF2 in the formation of the male germ cell cytoskeleton. Front. Cell Dev. Biol. 7, 151. https://doi.org/10.3389/fcell.2019.00151 (2019).

32. Starr, D. A. KASH and SUN proteins. Curr. Biol. 21, R414-R415. https://doi.org/10.1016/j.cub.2011.04.022 (2011).

33. Shao, X., Tarnasky, H. A., Lee, J. P., Oko, R. \& van der Hoorn, F. A. Spag4, a novel sperm protein, binds outer dense-fiber protein Odf1 and localizes to microtubules of manchette and axoneme. Dev. Biol. 211, 109-123. https://doi.org/10.1006/dbio. 1999.9297 (1999).

34. Shao, X., Xue, J. \& van der Hoorn, F. A. Testicular protein Spag5 has similarity to mitotic spindle protein deepest and binds outer dense fiber protein Odf1. Mol. Reprod. Dev. 59, 410-416. https://doi.org/10.1002/mrd.1047 (2001). 
35. Calvi, A. et al. SUN4 is essential for nuclear remodeling during mammalian spermiogenesis. Dev. Biol. 407, 321-330. https://doi. org/10.1016/j.ydbio.2015.09.010 (2015)

36. Pasch, E., Link, J., Beck, C., Scheuerle, S. \& Alsheimer, M. The LINC complex component Sun4 plays a crucial role in sperm head formation and fertility. Biol. Open 4, 1792-1802. https://doi.org/10.1242/bio.015768 (2015).

37. Yang, K., Adham, I. M., Meinhardt, A. \& Hoyer-Fender, S. Ultra-structure of the sperm head-to-tail linkage complex in the absence of the spermatid-specific LINC component SPAG4. Histochem. Cell Biol. 150, 49-59. https://doi.org/10.1007/s00418-018-1668-7 (2018)

38. Garcia-Higuera, I. et al. Folding of proteins with WD-repeats: comparison of six members of the WD-repeat superfamily to the G protein $\beta$ subunit. Biochemistry 35, 13985-13994. https://doi.org/10.1021/bi9612879 (1996).

39. Fong, H. K. W., Hurley, I. B., Doolittle, R. F. \& Simon, M. I. Repetitive segmental structure of the transducin Beta subunit homology with the CDC4 gene and identification of related mRNAs. Proc Natl. Acad. Sci USA 83, 2162-2166. https://doi.org/10.1073/ pnas.83.7.2162 (1986).

40. McClintock, T. S., Glasser, C. E., Bose, S. C. \& Bergman, D. A. Tissue expression patterns identify mouse cilia genes. Physiol. Genom 32, 198-206. https://doi.org/10.1152/physiolgenomics.00128.2007 (2008).

41. Stauber, M. et al. Identification of FOXJ1 effectors during ciliogenesis in the foetal respiratory epithelium and embryonic left-right organiser of the mouse. Dev. Biol. 423, 170-188. https:// doi.org/10.1016/j.ydbio.2016.11.019 (2017)

42. Burkhard, P., Stetefeld, J. \& Strelkov, S. V. Coiled coils: a highly versatile protein folding motif. Trends Cell Biol. 11, 82-88. https:// doi.org/10.1016/s0962-8924(00)01898-5 (2001).

43. Pazour, G. J., Agrin, N., Leszyk, J. \& Witman, G. B. Proteomic analysis of a eukaryotic cilium. J. Cell Biol. 170, 103-113. https:// doi.org/ $10.1083 /$ jcb. 200504008 (2005).

44. Blacque, O. E. et al. The WD repeat-containing protein IFTA-1 is required for retrograde intraflagellar transport. Mol. Biol. Cell 17, 5053-5062. https://doi.org/10.1091/mbc.F06-06-0571 (2006)

45. Fawcett, D. W. \& Phillips, D. M. The fine structure and development of the neck region of the mammalian spermatozoon. Anat. Rec. 165, 153-183. https://doi.org/10.1002/ar.1091650204 (1969).

46. Chemes, H., Carizza, C. \& Scarinci, F. Lack of a head in human spermatozoa from sterile patients: a syndrome associated with impaired fertilization. Int. J. Gynecol. Obstet. 26, 165-165. https://doi.org/10.1016/s0015-0282(16)50011-9 (1988).

47. Tang, S. et al. Biallelic mutations in CFAP43 and CFAP44 cause male infertility with multiple morphological abnormalities of the Tang, S. et al. Biallelic mutations in CFAP43 and CFAP44 cause male infertility with multiple morph
sperm flagella. Am. J. Hum. Genet. 100, 854-864. https://doi.org/10.1016/j.ajhg.2017.04.012 (2017).

48. Coutton, C. et al. Mutations in CFAP43 and CFAP44 cause male infertility and flagellum defects in trypanosoma and human. Nat. Commun. 9, 686. https://doi.org/10.1038/s41467-017-02792-7 (2018).

49. Kherraf, Z. E. et al. Homozygous ancestral SVA-insertion-mediated Deletion in WDR66 induces multiple morphological abnormalities of the sperm flagellum and male infertility. Am. J. Hum. Genet. 103, 400-412. https://doi.org/10.1016/j.ajhg.2018.07.014 (2018)

50. Göb, E., Schmitt, J., Benavente, R. \& Alsheimer, M. Mammalian sperm head formation involves different polarization of two novel

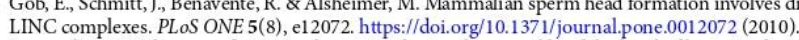
51. Laemmli, U. K. Cleavage of structural proteins during the assembly of the head of bacteriophage T4. Nature 227, 680-685. https
.//doiorg $/ 10.1038 / 22768020$ (1970).

52. Towbin, H., Staehelin, T. \& Gordon, J. Electrophoretic transfer of proteins from polyacrylamide gels to nitrocellulose sheets: Towbin, H., Staehelin, T. \& Gordon, J. Electrophoretic transfer of proteins from polyacrylamide gels to nitrocellulose sheets:
procedure and some applications. Proc. Natl. Acad. Sci. U. S. A. 76, 4350-4354. https://doi.org/10.1073/pnas.76.9.4350 (1979).

\section{Acknowledgments}

We thank Manfred Alsheimer, Würzburg, for the kind gift of antibodies. CTC is supported by grants from DAAD/CONICYT BECAS Chile program and Department of Developmental Biology, GZMB, Georg-AugustUniversität Göttingen, Germany.

\section{Author contributions}

Conceived and designed experiments: S.H.-F. Performed experiments: C.T.C. Prepared figures and wrote the manuscript: S.H.-F. and C.T.C. Both authors read and approved the final manuscript.

\section{Funding}

Open Access funding provided by Projekt DEAL.

\section{Competing interests}

The authors declare no competing interests.

\section{Additional information}

Supplementary information is available for this paper at https://doi.org/10.1038/s41598-020-71120-9.

Correspondence and requests for materials should be addressed to S.H.-F.

Reprints and permissions information is available at www.nature.com/reprints.

Publisher's note Springer Nature remains neutral with regard to jurisdictional claims in published maps and institutional affiliations.

(c) (i) Open Access This article is licensed under a Creative Commons Attribution 4.0 International format, as long as you give appropriate credit to the original author(s) and the source, provide a link to the Creative Commons license, and indicate if changes were made. The images or other third party material in this article are included in the article's Creative Commons license, unless indicated otherwise in a credit line to the material. If material is not included in the article's Creative Commons license and your intended use is not permitted by statutory regulation or exceeds the permitted use, you will need to obtain permission directly from the copyright holder. To view a copy of this license, visit http://creativecommons.org/licenses/by/4.0/

(c) The Author(s) 2020 


\section{Discussion}

\subsection{Relevance and impact of mutations of candidate genes involved in the linkage of sperm tail to its head}

Infertility has been defined by the International Committee for Monitoring Assisted Reproductive Technology, World Health Organization (WHO), as a reproductive system disease defined by the failure to achieve a clinical pregnancy after 12 months or more of regular unprotected sexual intercourse (Zegers-Hochschild et al., 2009). This condition is a global issue and it affects approximately $15 \%$ of couples due to primary or secondary infertility. Thus, around 48.5 million couples worldwide suffer from infertility. At least $20 \%-30 \%$ and up to $40 \%$ of reproduction failure is based on male infertility (WHO, 2010; Sharlip et al., 2002; Sharma, 2017).

Male infertility has attracted more and more attention, along with the development of assisted reproductive technology (ART). Hence, identifying the causative factors of sperm pathologies, especially when they originate genetically, is mandatory for successful ART. Therefore, understanding the genetic causes of male infertility contribute to the efficacy, safety, and health of the progeny conceived by ART (Sharlip et al., 2002; Sharma, 2017).

Teratozoospermia is defined as a low percentage of spermatozoa with normal morphology (WHO, 2010). The acephalic spermatozoa syndrome is a type of teratozoospermia caused by an abnormal head-tail connection that arises during spermiogenesis (Chemes \& Alvarez Sedo, 2012; Miyamoto et al., 2012). Moreover, the decapitated sperm syndrome has been reported in familial cases suggesting a genetic origin (Baccetti et al., 1989; Chemes et al., 1999). However, which genes are affected is still unclear.

Alterations of the head-neck attachment have been described in mice provoked by the experimental depletion of specific genes. Among these genes, Odf1 and Spag4l are interesting candidates to analyze in humans because their disruption in mice produces relaxation of the head-tail junction and sperm decapitation (Yang et al., 2014; Yassine et al., 2015). Furthermore, in infertile men some Spag4l mutations have already been identified in infertile men with decapitated spermatozoa (Elkhatib et al., 2017; Zhu et al., 2016).

ODF1 is encoded by two slightly different alleles giving rise to two proteins that differ in the number of the C-terminal tripeptide motifs, CSP or CNP (Hofferbert et al., 1993). Both alleles 
were also found in the two patients analyzed here. However, the variation of the expression of these alleles is not causative for spermatozoa decapitation. Beyond that, no additional variations were found in the coding region of the Odfl-gene in the two patients studied here.

The two exons that encode ODF1 are separated by a $7 \mathrm{~kb}$ intron (Hofferbert et al., 1993). Even though intronic sequences do not encode a protein, they contain conserved regulatory elements involved in pre-mRNA splicing. Thus, mutations in intronic regions might also alter gene expression (Gilbert 1978; Kelly et al., 2015). It is the case of the intronic mutation c.425 $+1 \mathrm{G} \rightarrow \mathrm{A}$ that affects the Sun5-gene leading to a premature stop codon (Zhu et al., 2016). So far, no mutations have been reported to affect either Odfl exons or intron. Nonetheless, exploring intronic variants that potentially affect $O d f 1$-gene expression might be highly informative.

Since a mutation in $O d f 1$ was excluded, I turned to $S p a g 4 l$ as the next likely candidate. Based on the study conducted by Zhu et al. (2016), specific mutations in 8 of 17 infertile male patients were identified. The mutations described in that study affected the exons 4, 5, 6, 7, 8, 11 and 13. Therefore, I attempted to investigate these variants in two unrelated patients with the acephalic spermatozoa syndrome. The exons 6,8 were successfully amplified, though their sequences displayed no differences compared to the wild-type sequence. In contrast, the amplification of exons 5, 11, and 13 failed due to unknown reasons, albeit the same primers were used as indicated by Zhu et al. (2016).

The unsuccessful identification of mutations in the gene Sun5 in the patients studied here might be related to the inappropriate selection of exons. I centered the attention on the exons 6-8 and 11-13 because they encode either the coiled-coil or SUN domain of SUN5, respectively (Zhu et al., 2016). Although mutations in exons 6 and 8 have been reported in patients with decapitated spermatozoa (Zhu et al., 2016), they are not causative of the syndrome in the patients studied here.

Besides that, Hook1 attracted my attention because a truncating mutation caused abnormal head shape and sperm decapitation in mice (Mendoza-Lujambio et al., 2002). Further, the missense mutation p.Q286R in exon 10 has been reported in infertile men that suffer from headless sperm (Chen et al., 2018). Moreover, HOOK1 localizes in the manchette, which is essential for the transport of molecules required for tail formation (Mendoza-Lujambio, 2002). Therefore, I investigated the Hookl gene in two unrelated patients with acephalic spermatozoa syndrome. Since deletion of exons 10 and 11 in mice caused $a z h$ (abnormal spermatozoon head shape) phenotype, and mouse exon 10 corresponds to the human exon 10, I concentrated on these 
regions. Further, exon 10 encodes the VWFC domain that seems to be involved in the formation of protein complexes (Bork 1993). However, I found no evidence for mutation by sequencing the clones of the PCR products.

Our mutation screening using the genomic DNA of two unrelated infertile men that suffer from sperm decapitation excluded mutations in the coding region of $O d f 1$ as causative. Furthermore, I found no indications for mutation in exon 6 of Spag4l or in exons 10 and 11 of Hookl. The acephalic spermatozoa syndrome is a rare condition. Therefore, the failure in the identification of a mutation in the two patients investigated is not surprising. To demonstrate a causative mutation requires careful inspection of a large number of patients and genes. Besides that, Zhu et al. (2016) has described an intronic mutation that affects the coiled-coil structure of the SUN5. Therefore, it remains to explore the intronic sequences of the genes Odfl and Hookl that eventually affect the protein conformation. In mice, some genes have been identified as causative of sperm decapitation phenotype, but mutations affecting these genes in humans have rarely been demonstrated. The loss of function of $C c d c 42$ in mice severely affects the formation of the HTCA. However, no mutations in $C c d c 42$ were found in these two human patients (Hector Chemes, personal information).

A high number of genes are involved in male infertility, which not only disrupts male germ cells but also may cause genetic impairments in the offspring. These gene mutations may be autosomal recessive or dominant and highly variable between individuals (Hackstein et al., 2000; Matzuk et al., 2002). Even though many genes are involved in male infertility, few affect spermiogenesis (Vogt, 2004). Therefore, the individual's genetic constitution and the few known genes that affect the head-tail junction hamper the detection of mutations in patients. Here, I investigated specific regions of three promising candidate genes. However, identifying the cause of the sperm decapitation in the two patients analyzed requires an intensive screening of mutations in still unexplored genes. Further, DNA sequencing from infertile and fertile male patients that share a specific genetic background may enable identifying new genes involved in the acephalic spermatozoa syndrome.

Considering the complexity of the factors associated with male infertility and the limitation of performing studies in many patients, mouse models are particularly valuable. Therefore, to evaluate the impact of SUN5 on the spermatozoa ultrastructure, Spag4l-deficient mice were developed. Two female founders were mated with wild-type males. After genotyping of the offspring by PCR, $~ 38 \%$ of the F1 animals were heterozygous that is below the expected Mendelian ratio of 50\%. Heterozygous F1 mice were mated with wild-type mice. Intriguingly, we obtained only a $\sim 9 \%$ of heterozygous mice in F2 generation, whereas the expected number 
of heterozygous mice was 50\%. Spag $4 l^{+/}$mice obtained from two independent founder animals were mated to obtain the F3 and F4 generations. Around 30\% of heterozygous mice were detected by genotyping, albeit they carried only a small fragment of the integration cassette, but no homozygous Spag4l-deficient mice.

The gradual reduction of the cassette size over the generations was intriguing. In ES cells, the up and downstream regions of the recombinant allele were detected by genotyping, demonstrating the correct integration of the replacement cassette into the Spag4l gene. Afterward, only the resistant gene neomycin and the flanking region at the 3 -terminal were amplified. However, in further generations, only a small fragment of the neomycin insertion was amplified, whereas the amplification of the flanking regions and the reporter gene LacZ failed.

Multiple biological mechanisms may cause an unusual transmission ratio, including a faulty integration of the targeting allele into the genome, genomic instability of the ES cell, and late injection of ES cells into blastomeres (Martin, 1981; Palmiter et al., 1982). First, correct incorporation of the insertion is fundamental to ensure the stable transmission of the modified allele to the offspring (Palmiter et al., 1982). Further, if transgenic cells' contribution to the germline is low, the foreign DNA transmission frequency will be greatly reduced (Wilkie et al., 1986). Therefore, ES cell clones were carefully analyzed, and the sequencing of the PCR product confirmed the correct position of the cassette. The integration of the cassette replaced nearly the complete Sun5-gene, leaving only $89 \mathrm{bp}$ of the N-terminal region. However, the founders were not able to fully transmit the transgene.

Moreover, females are mostly undesirable for a breeding scheme, only two female chimeras tested positive for the transgene and were selected as founders. Among the disadvantages of female founders are the duration of the breeding process as well as the uncertainty of a successful germline transmission (Longenecker et al., 2009). Even though female founders produced offspring, they did not efficiently transmit the cassette. Besides that, the first two generations were tested by nested PCR, given that the amplification of the 5 - region of the insertion cassette could not be amplified. Thus, it is possible that our genotyping results of F1 and further generations were mostly false positive. 


\subsection{Topology and localization of SPAG4 and SPAG4L2 at the nuclear membrane might mediate the connection of the sperm nucleus to its tail}

SPAG4 and SPAG4L/SPAG4L2 are members of the mammalian SUN domain protein family and reside in the nuclear membrane. The mammalian SUN domain protein family comprises at least five conserved SUN-domain proteins. SUN1 and SUN2 are widely expressed in somatic and germinal cells, while SUN3, SUN4/SPAG4, and SUN5/SPAG4L are restricted to male germ cells (Malone et al., 1999; Göb et al., 2010; Padmakumar, 2005; Frohnert et al., 2011). The SUN domain protein family shares a similar structure, consisting of an $\mathrm{N}$-terminal region containing up to three transmembrane domains (TMs), followed by one or two coiled-coil regions and a conserved SUN domain at the C-terminal region. The $\mathrm{N}$-terminus containing the transmembrane domains spans the inner nuclear membrane and interacts with the nuclear lamina and chromatin. Meanwhile, the C-terminus containing the SUN-domain resides in the perinuclear space. Here, the SUN-domain interacts with the KASH domain proteins located in the ONM, thus linking the cytoskeleton to the nucleoskeleton (Hodzic et al., 2004; Crisp et al., 2006; Haque et al., 2006; Razafsky \& Hodzic, 2009)

In round spermatids, SPAG4L is recruited from the cytoplasm to the nuclear envelope except for the NE overlaying the acrosome. Meanwhile, in elongating spermatids, SPAG4L as well as SPAG4 locate to the posterior pole of the NE, and more specifically at the implantation fossa (Yassine et al., 2015; Yang et al., 2018). Previous analyses have confirmed that SPAG4 and SPAG4L interact with ODF1 in vitro (Shao et al., 1999; 2001). Further, the homozygous mutation c.381delA that affects the TM of Sun5 in humans seems to change the expression and distribution of ODF1 (Sha et al., 2018). This data indicates that SPAG4 and SPAG4L are involved in the recruitment of ODF1 to the nuclear membrane. However, the localization of SPAG4 and SPAG4L at the NE does not explain their ability to interact with ODF1 that locates in the basal plate and capitulum. Therefore, this raises the question of whether the N-terminal region of SPAG4 might also localize at the outer nuclear membrane to enable the interaction with cytoskeletal proteins, as e.g. ODF1.

To obtain a clearer view of the orientation of SPAG4 and SPAG4L within the NE, their transmembrane segments and domains were analyzed. The localization pattern of different regions and domains of SPAG4L revealed a clear localization of TM1 and TM2 in the NE. TM1 also locates in the cytoplasm, and TM2 strongly concentrates in the area that seems to be the ER. In contrast to TM2, TM1 is widely distributed to the nuclear envelope and cytoplasm, whereas the combination of both TMs recruits the protein to the nuclear envelope. These results indicate that the TM1 is responsible for the recruitment of the protein to the nuclear membrane. 
In contrast, the presence of the TM2 in the Golgi-apparatus might be associated with protein trafficking through membrane compartments. Analyses of the dynamics of SUN5 during spermiogenesis have indicated that SUN5 transits through the Golgi apparatus to experience post-translational modifications before reaching its final localization at the NE (Yassine et al., 2015). Additional data also indicate that TM2 does not recruit SPAG4L to the nuclear membrane. The mutation c.340G $\rightarrow$ A (p.Gly114Arg) affects the TM-domain of Spag4l in humans, whereas in mice the mutation localizes in the position c.334G>A (p.Gly114Arg) and affects the TM2 of Spag4l2. The overexpression of SUN5 carrying the p.Gly114Arg mutation in HeLa cells induces protein aggregation near the NE but does not prevent its localization at the nuclear membrane (Elkhatib et al., 2017; Shang et al., 2018).

The results also indicate that the coiled-coil and SUN domains are not involved in retaining SPAG4L at the NE. According to a previous study, the deletion of the SUN-domain coding region of Spag4l (exons 10, 11, and 12) leads to male infertility in mice (Shang et al., 2017). Therefore, I investigated whether the C-terminal truncation of SPAG4L2 affects its nuclear membrane localization. My results show that the truncated SPAG4L2 is localized in the nuclear membrane with a similar distribution as the full-length protein. These results coincide with Frohnert et al. (2011), who described the two TMs (TM1 and TM2) as mediators of SPAG4L2 recruitment to the NE. Besides, Hasan et al. (2006) and Hodzic et al. (2004) have also reported that the SUN-domain is not responsible for maintaining SUN1 and SUN2 at the NE, confirming the data obtained here.

These data suggest that the TM1 plays a major role in recruiting SPAG4L and SPAG4L2 at the NE. Thus, mutations affecting the coding sequence of TM1 and TM2 might impair nuclear membrane localization. On the other hand, mutations affecting the SUN-domain might disrupt the ability of SPAG4L2 to establish protein interactions, thus affecting the linkage to the cytoskeleton and perhaps the linkage of the sperm head to the tail. However, it remains the question how SPAG4 and SPAG4L interact with cytoplasmatic proteins.

Since SPAG4 and SPAG4L are nuclear membrane proteins in somatic cells and SUN proteins form the LINC complex, it is suggested that SPAG4 or SPAG4L might connect the NE to the capitulum via ODF1. For this reason, the orientation of the N-terminal region of SPAG4 and SPAG4L acquire great relevance and might explain the mechanism of these proteins to interact with ODF1. Thus, I used the rapamycin-based FKBP-FRB dimerization to analyze the orientation of SPAG4 and SPAG4L. 
Rapamycin induces the formation of a ternary complex between FKBP and FRB (Banaszynski et al., 2005). Therefore, the binding domain FRB was coupled to the N-terminus of SUN4 (or SUN5)-ECFP, whereas Luciferase-mRFP was coupled to FKBP to its C-terminus. LuciferasemRFP-FKBP was mostly distributed at the cytoplasm, whereas FRB-ECFP-SUN4 (SUN5) localized at the nuclear membrane. The addition of rapamycin induced the recruitment of Luciferase-mRFP-FKBP from the cytoplasm to the nuclear envelope.

Thus, the $\mathrm{NH}_{2}$-termini of SPAG4 and SPAG4L containing FRB were able to interact with their partner FKBP in the presence of rapamycin. Without rapamycin induction, Luciferase-mRFPFKBP was exclusively cytoplasmatic. These results indicate that the N-terminal region of SPAG4 and SPAG4L was oriented to the outer nuclear membrane (ONM). On the contrary, if the $\mathrm{NH}_{2}$-termini of SPAG4 and SPAG4L were localized at inner nuclear membrane INM, FRB would not be able to recruit the cytoplasmic Luciferase to the nuclear envelope.

To sum up, these results support the view that the $\mathrm{NH}_{2}$-termini of the $\mathrm{SUN}$-domain proteins SPAG4 and SPAG4L localize at the ONM as well as the INM. Thus, this model could explain the ability of SPAG4 and SPAG4L to interact with ODF1 and still unknown cytoplasmatic proteins to generate the coupling apparatus in sperm (Fig. 4.2, B).

So far, the only known interaction partner of SPAG4 is the HTCA protein ODF1, indicating its function in the establishment of a novel sperm-specific LINC complex. Additional SPAG4 interacting partners that collaborate in the formation of the connecting piece still await to be identified. Therefore, I have explored the application of the amber suppression strategy to identify in vitro new SPAG4 interacting partners that might collaborate in the formation of the HTCA. To identify potential protein interactions, it is necessary to overexpress candidate testisspecific proteins in somatic cells in combination with an engineered sequence able to encode a photoreactive amino acid.

The amber suppression method works by the reassignment of the stop-codon function that enables the expansion of the genetic code. Stop codons can encode unnatural amino acids (UAAs) and be incorporated into proteins. Further, the UAAs serve to label proteins to investigate their functionality in a native context (Xie \& Schultz, 2005). The translation of UAAs can incorporate photo-crosslinking amino acids, e.g., p-benzoyl-phenylalanine ( $p$ Bpa). Once the amino acid $p \mathrm{Bpa}$ has been integrated into the protein chain, cross-linking to close $\mathrm{C}$ $\mathrm{H}$ bonds of nearby proteins can be induced by illumination with UV-light of 350-360 nm (Kauer et al., 1986). Thus, the cross-linking of close-by proteins or peptides enables the identification of potential interacting proteins (Chin et al., 2002, 2003; Hino et al., 2005; Xie \& Schultz, 2005). 
In this study, I evaluated the feasibility of this method to identify LMNC interacting partners. First, the amber stop codon was incorporated into three different regions of LMNC, giving rise to three independent LMNC expression plasmids. Amber nonsense mutations were inserted into the C-terminal region of LMNC because this region contains immunoglobulin domains, which are supposed to be involved in protein-protein interactions (Dechat et al., 2000). Once the amber codon replaced the amino acid Arg-470, Thr-488, and Thr-534 in the LMNC sequence respectively, the amber codon was translated into the photo-activatable amino acid ( $p \mathrm{Bpa}$ ) in the presence of the orthogonal tRNA-aaRS (p4XtRNA $p$ Bpa) and $p$ Bpa.

The correct incorporation of the unnatural amino acid in LMNC was confirmed immunocytologically. Cells that were exclusively transfected with Lmnc containing the nonsense sequence produced a truncated protein. Thus, LMNC was detected in the nucleoplasm forming aggregates. The incorporation of $p \mathrm{Bpa}$ restored LMNC localization at the nuclear membrane. Considering that the use of the inducible crosslinking of LMNC containing a UAA can react with native proteins, it is mandatory to validate the system and avoid false positives. Lmnc was co-transfected with Spag4, confirming that their respective proteins do not interact. However, unspecific bands were detected by Western blotting. These protein bands could be explained by the unspecificity of the antibodies and the presence of endogenous LMNC expressed in NIH3T3 cells detected by the anti-laminA/C antibody.

To further evaluate the ability of the modified LMNC to interact with exogenous proteins, Lmnc was transfected with its known binding partners Lmnb3 and Lmnc2, respectively (Ye \& Worman, 1995). However, no cross-linking between these proteins was found, whereas LMNB3 and LMNC2 could be detected (data are not shown).

These results show that the amber suppression method is sensitive and susceptible to several experimental conditions, including the amount of RS/tRNA transfected and the appropriate protein translation. Furthermore, the modified LMNC is highly reactive with endogenous proteins complicating the detection of interacting proteins.

Even though this system is appropriate to detect weak and transient interactions with high specificity in mammalian cells (Hino et al., 2005), the identification of testicular protein interactions will only be possible by their ectopic expression. However, the protein that will be engineered to induce the photo-crosslinking should be carefully chosen to fit the specific purpose of the experiment. Considering this aspect, the amber suppression method should first be evaluated using the known interacting partners LMNA and SUN1 and SUN2 (Haque et al., 2006; Crisp et al., 2006). Furthermore, LMNA is a suitable candidate to evaluate its interaction 
with the testes specific SUN-domain proteins SUN3, SUN4 and SUN5. For this goal, a sitespecific UAA incorporation into Lmna and the addition of a tag for its posterior detection by Western-blotting is mandatory. LMNA contains the immunoglobulin Ig-fold in its C-terminal region, which may mediate specific intermolecular protein-protein, protein-DNA, and proteinphospholipid interactions (Dhe-Paganon et al., 2002). It is known that the region of LMNA that encompasses residues 430-545 adopts an Ig-like fold (Krimm et al., 2002). Therefore, this area is an interesting "hot-spot" to incorporate $p$ BPA. Moreover, the modification of the amino acid R482 (R482W) alters the interaction of the LMNA with LAP2 $\alpha$ and emerin, among others (Gilchrist et al., 2004). Hence, it will be recommended to consider replacing the amino acid R482 with the amber stop codon.

\subsection{Identification of proteins that collaborate in the formation of the HTCA}

The HTCA formation and its structure have acquired great relevance because its proper development ensures oocyte fertilization. The ultrastructure of the HTCA is formed by specific components such as the basal plate, capitulum, centrioles, and segmented columns. However, its molecular components are barely known.

ODF1 is a pivotal component of the HTCA, and its disruption in mice affects the thigh junction of the sperm head-to-tail, causing sperm decapitation. It localizes in the cytoskeletal portion of the HTCA (Yang et al., 2012; 2014), whereas its interacting partner SPAG4, localizes at the nuclear membrane of the sperm (Shao and van der Hoorn, 1996; Shao et al., 1997; 1999). Considering that the linkage between the nucleoskeleton and the cytoskeleton in somatic cells is mediated via interaction between SUN domain and KASH domain proteins, the HTCA might be established via interaction between male germ cell-specific SUN domain proteins and still to be identified KASH domain proteins or directly to cytoskeletal proteins, as, e.g., ODF1 (Starr, 2002; Starr \& Fridolfsson, 2010). The SUN-domain proteins reside at the nuclear membrane. Therefore, the ability of SPAG4 and SPAG4L to interact with cytoskeletal proteins in sperm is far from being comprehended. SPAG4 and SPAG4L contain at least one transmembrane domain responsible for recruiting the full-length protein to the nuclear envelope. Further, from the five SUN-domain proteins encoded in mammals, SUN1 and SUN2 reside at the INM (Hodzic et al., 2004; Haque et al., 2006; Hasan et al., 2006). However, the data obtained here indicated that SPAG4 and SPAG4L also localize at the ONM. This suggests that the N-terminal region of SPAG4 and SPAG4L might recruit ODF1 to the nuclear membrane and thus link the sperm head to its tail (Fig. 4.2, B). Furthermore, the Sun5 mutation c.381delA (p.Val128Serfs*7) has 
been described in $33.33 \%-47.06 \%$ of Chinese patients affected by the acephalic spermatozoa syndrome. Particularly, this mutation localizes at the coding region of the transmembrane domain and reduces the expression of ODF1 in sperm from an infertile patient compared to the sperm control (Sha et al., 2018). In Drosophila, SPAG4 is involved in the linkage of the sperm head to the flagellum through the coiled-coil protein Yuri Gagarin. Yuri is a component of the basal body in elongated spermatids and its localization is altered by the absence of SPAG4 (Kracklauer et al., 2010). These data support the ability of SPAG4 and SPAG4L, to recruit cytoskeletal proteins such as ODF1 to the nuclear envelope.

ODFs are prominent components of the cytoskeletal structure of the sperm tail, and mostly located in the mid-piece of human spermatozoa (Oko, 1988; Petersen et al., 1999). From these proteins, the major components in human spermatozoa are ODF1, ODF2, and ODF3 (Petersen et al., 2002). ODF1 and ODF2 are interacting partners via their leucine zipper motif (Shao et al., 1997), whereas ODF3 is a cytoskeletal coiled-coil protein (Petersen et al., 2002). ODF1 (unpublished observation) and ODF2 are centrosomal components in somatic cells (Schweizer et al., 2009), and both are required to maintain a tight connection between the sperm head and its tail (Yang et al., 2012, 2014; Ito et al., 2019). These proteins are components of the cellular cytoskeleton and their structure may contribute to forming a scaffold of proteins involved in the formation of the HTCA. ODF3, also known as PMFBP1 or STAP, has a reported effect in the detachment of the sperm nuclear envelope to the connecting piece when it is faulty (Sha et al., 2019). In humans, six mutations that affect Odf3/Pmfbpl have been identified to be causative of the acephalic spermatozoa syndrome (Zhu et al., 2018; Sha et al., 2019). In humans and mice spermatozoa, polyamine-modulated factor 1-binding protein 1 "PMFBP1" localizes at the HTCA, specifically between SUN5 and SPATA6 (Zhu et al., 2018). SUN5 locates at the NE, whereas SPATA6 in the segmented columns and capitulum (Frohnert et al., 2010; Yuan et al., 2015). Thus, the complex formed by SUN5/PMFBP1/SPATA6 contributes to the junction of the sperm head to the tail. Moreover, the mutations c.425+1G $>$ A and c.1043A $>$ T in Sun5 affect the localization of PMFBP1 in the implantation fossa, indicating their cooperative role in the HTCA formation (Zhu et al., 2018). The coiled-coil protein ODF3/PMFBP1/STAP may serve as platform of still unidentified protein that collaborate in the in the articulation of the sperm head to the tail.

Another protein also implicated in the formation of the HTCA is the coiled-coil domaincontaining protein 42 (CCDC42). Genetic depletion of CCDC42 in mice affects sperm nuclear shaping and the implantation site leading to male infertility (Pasek et al., 2016). Our subcellular analyses have detected CCDC42 in the manchette and perinuclear ring in elongating spermatids, 
whereas, in sperm, it localizes to the connecting piece and the sperm tail. The presence of CCDC42 in the manchette and sperm tail coincides with the localization of ODF1. Moreover, in somatic cells, ODF1 and CCDC42 colocalized in the centrosome, indicating an interaction between both proteins. The interaction between CCDC42 and ODF1 as well as ODF2 was probed by pull-down and Co-IP assays. These data indicate that the interaction between CCDC42 and ODF1 and ODF2 might serve as a scaffold in forming the HTCA and the sperm tail.

The current research has shown that CCDC42 and ODF1 as well as the coiled-coil protein HOOK1 localize at the microtubular manchette. Depletion of Hookl disrupts the formation of the HTCA leading to sperm decapitation (Mendoza-Lujambio, 2002). HOOK1 interacts with the manchette-associated protein RIMBP3. Disruption of Rimbp3 results in abnormal sperm head shape, detached acrosome, abnormal manchette positioning, and disarranges the sperm head-to-tail linkage (Zhou et al., 2009). Furthermore, RIM-BP3 interacts with the microtubuledependent motor protein KIF3B which is involved in the manchette function and sperm tail formation. KIF3B seems to be involved most likely in the intra-manchette transport of proteins towards the axoneme (Zhou et al., 2009). KIF3B forms a complex with KIF3A, another microtubule-based motor member of the kinesin family (Yamazaki et al., 1995). KIF3A localizes in the basal body, axoneme and manchette of elongating spermatids, whereas in mature spermatozoa it situates in the sperm tail (Lehti et al., 2013). In mice spermatids, KIF3A disruption affects the nuclear shaping, manchette organization and alters the axoneme organization and tail accessory structures (Lehti et al., 2013). Thus, KIF3A seems to be involved in the microtubule network of the manchette and flagellar assembly (Lehti et al., 2013). During axoneme formation, Kif3a expression correlates with the expression of Odf3/Pmfbpl and Ccdc11/Cfap53 suggesting their interaction and collaboration in the formation of the flagellum (Lehti et al., 2013).

As another putative component of the HTCA, the cilia and flagella associated protein 52 (CFAP52) came into focus. CFAP52/WDR16 is a WD-repeat domain protein, which contains GH-WD repeats. These repetitive motifs have the property to support protein-protein interaction enabling CFAP52 to perform several biological functions. WD-repeat domain proteins are involved in RNA synthesis/processing, signal transduction, cytoskeleton assembly, mitotic spindle formation, vesicular trafficking, and cell growth (Neer et al., 1994; Li \& Roberts, 2001). Therefore, I was interested first in the expression and localization of CFAP52 in somatic and male germ cells. I demonstrated for the first time that CFAP52 is a centrosomal protein in somatic cells. Furthermore, it localizes to the basal body, the mitotic spindle poles, and the 
intercellular bridge. In male germ cells, CFAP52 is a component of the manchette and sperm tail as well as ODF1, although these proteins do not interact. Therefore, these results suggest that CFAP52 might be associated with microtubular structures and is involved in the traffic of proteins through the manchette. These data indicate that CFAP52 may be a suitable interacting partner of KIF3A which is also involved in the manchette and axoneme organization (Fig. 4.1).

In summary, ODF1 and CCDC42 are essential for HTCA formation since their deficiency causes sperm decapitation. Disruption of SPAG4 debilitates the tight junction between the sperm tail and head. SPAG4L is responsible for the attachment between the sperm nucleus and the flagellum, and mutations lead to the acephalic syndrome in humans. The proteins CFAP52 and CCDC42 were identified as centrosomal proteins in somatic cells and resided in specific structures of male germ cells where they colocalize with ODF1 (Fig. 4.2, A). These proteins may form a complex to stabilize the connecting piece, and their failure leads to sperm decapitation and thus human male infertility.

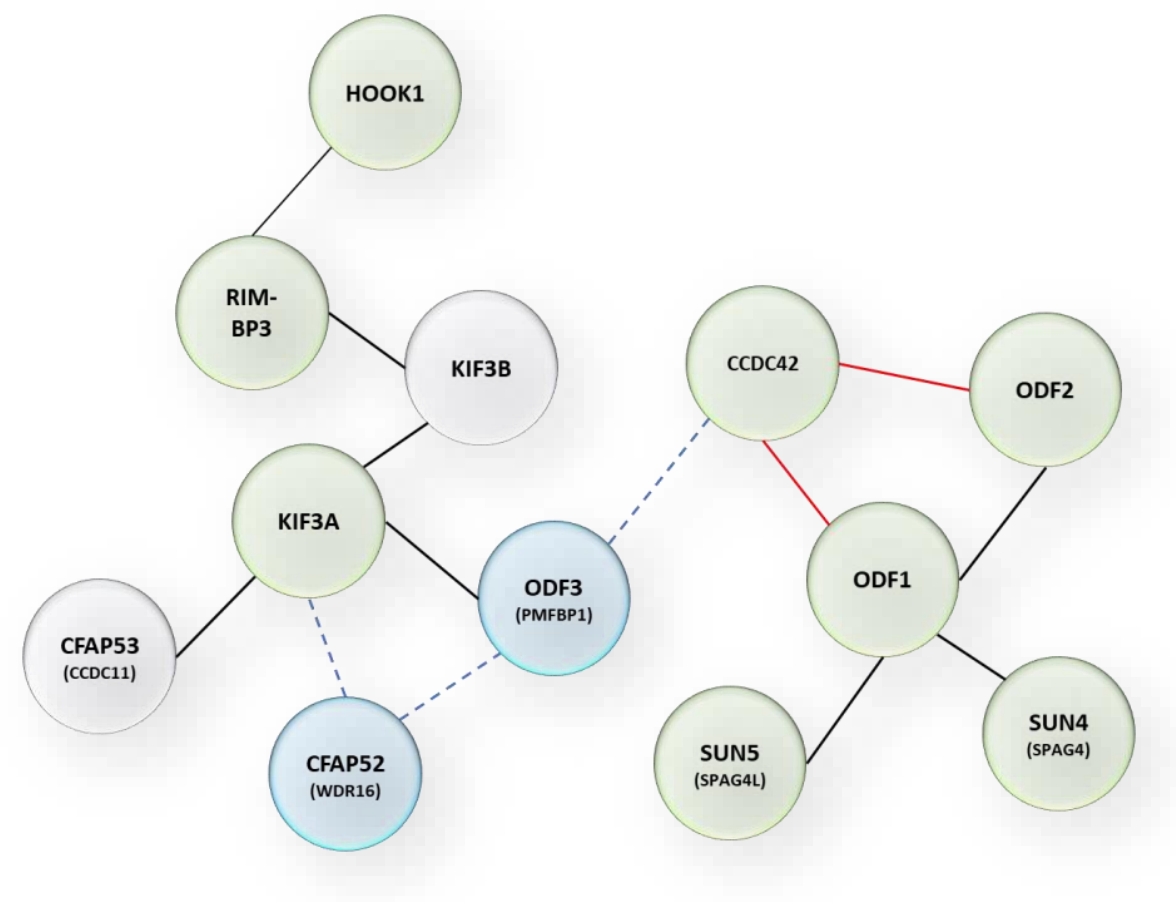

Figure 4.1. Mapping of HTCA protein interaction network. Green nodes represent proteins with a reported effect in the formation of the HTCA. Blue nodes represent structural proteins in mammalian spermatozoa. Gray nodes represent proteins associated with microtubular structures in mitotic or meiotic cells. Red lines represent the interactions identified in this study. Black lines represent proteins interactions previously published. Light blue dashed lines represent potential protein interactions. 


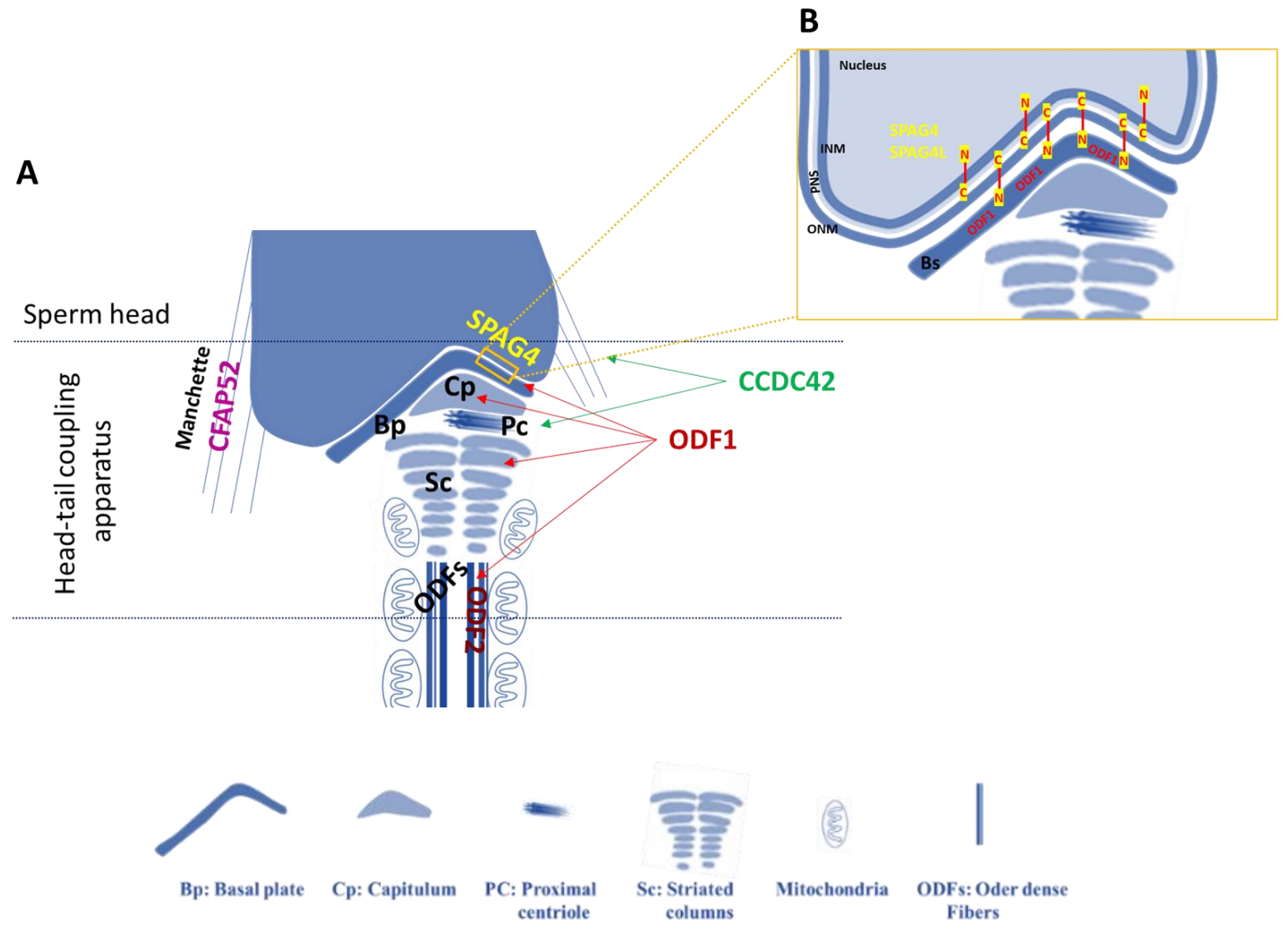

Figure 4.2. Representative scheme of proteins associated with the HTCA in elongating spermatids.

(A) The HTCA is composed of the basal plate $(\mathrm{Bp})$, capitulum $(\mathrm{Cp})$, proximal centriole (Pc) and striated columns ( $\mathrm{Sc}$ ). The sperm head is linked to the connecting piece via basal plate and capitulum. SPAG4 localizes at the nuclear envelope and interacts with the cytoskeletal protein ODF1. ODF1 localizes at the basal plate, capitulum, striated columns and ODFs. ODF1 interacts with ODF2 and CCDC42. CCDC42 localizes to the manchette and connecting piece. CFAP52 is a component of the manchette. (B) SPAG4 and SPAG4L localizes at the nuclear membrane. ODF1 localizes at the basal plate. The N-terminal region of SPAG4 and SPAG4L might be oriented towards the inner nuclear membrane (INM) or the outer nuclear membrane (ONM). In contrast, their C-terminal region localizes at the perinuclear space (PNS). The N-terminal region of SPAG4 and SPAG4L oriented to the ONM might enable the interaction with the cytoskeletal protein ODF1. 


\section{$5 \quad$ References}

Alsheimer, M., von Glasenapp, E., Schnolzer, M., Heid, H., \& Benavente, R. (2000). Meiotic lamin C2: The unique amino-terminal hexapeptide GNAEGR is essential for nuclear envelope association. Proceedings of the National Academy of Sciences, 97(24), 1312013125. https://doi.org/10.1073/pnas.240466597

Alsheimer, M. \& Benavente, R. (1996). Change of Karyoskeleton during Mammalian Spermatogenesis: Expression Pattern of Nuclear Lamin C2 and Its Regulation. Experimental Cell Research, 228(2), 181-188. https://doi.org/10.1006/excr.1996.0315

Alvey, P. L. (1985). An investigation of the centriole cycle using $3 \mathrm{~T} 3$ and CHO cells. Journal of Cell Science, 78(1), 147-162. https://jcs.biologists.org/content/78/1/147

Avidor-Reiss, T., Mazur, M., Fishman, E. L., \& Sindhwani, P. (2019). The Role of Sperm Centrioles in Human Reproduction - The Known and the Unknown. Frontiers in cell and developmental biology, 7, 188. https://doi.org/10.3389/fcell.2019.00188

Baccetti, B., Burrini, A. G., Collodel, G., Magnano, A. R., Piomboni, P., Renieri, T. \& Sensini, C. (1989). Morphogenesis of the decapitated and decaudated sperm defect in two brothers. Gamete Research, 23(2), 181-188. https://doi.org/10.1002/mrd.1120230205

Balhorn, R. (1982). A model for the structure of chromatin in mammalian sperm. The Journal of Cell Biology, 93(2), 298-305. https://doi.org/10.1083/jcb.93.2.298

Baltz, J. M., Oneeka Williams, P. \& Cone, R. A. (1990a). Dense Fibers Protect Mammalian Sperm Against Damage. Biology of Reproduction, 43(3), 485-491. https://doi.org/10.1095/biolreprod43.3.485

Baltz, J. M., Oneeka Williams, P. \& Cone, R. A. (1990b). Dense Fibers Protect Mammalian Sperm Against Damage. Biology of Reproduction, 43(3), 485-491. https://doi.org/10.1095/biolreprod43.3.485

Banaszynski, L. A., Liu, C. W., Wandless, T. J. (2005). Characterization of the FKBP.rapamycin.FRB ternary complex. Journal of the American Chemical Society, 127(13), 4715-4721. https://doi:10.1021/ja043277y

Bhullar, B., Zhang, Y., Junco, A., Oko, R. \& van der Hoorn, F. A. (2003). Association of Kinesin Light Chain with Outer Dense Fibers in a Microtubule-independent Fashion. The Journal of biological chemistry, 278(18), 16159-16168. https://doi.org/10.1074/jbc.M213126200 
Bork, P. (1993). The modular architecture of a new family of growth regulators related to connective tissue growth factor. FEBS letters, 327(2), 125-130. https://doi.org/10.1016/0014-5793(93)80155-n

Bornens, M. (2002). Centrosome composition and microtubule anchoring mechanisms. Current Opinion in Cell Biology, 14(1), 25-34. https://doi.org/10.1016/S0955-0674(01)00290-3

Braun, R. E. (1990). Temporal translational regulation of the protamine 1 gene during mouse spermatogenesis. Enzyme, 44(1-4), 120-128. https://doi.org/10.1159/000468752

Burfeind, P. \& Hoyer-Fender, S. (1991). Sequence and developmental expression of a mRNA encoding a putative protein of rat sperm outer dense fibers. Developmental Biology, 148(1), 195-204. https://doi.org/10.1016/0012-1606(91)90329-2

Burke, B. \& Stewart, C. L. (2002). Life at the edge: The nuclear envelope and human disease. Nature Reviews Molecular Cell Biology, 3(8), 575-585. https://doi.org/10.1038/nrm879

Burke, B. \& Stewart, C. L. (2013). The nuclear lamins: Flexibility in function. Nature Reviews. Molecular Cell Biology, 14(1), 13-24. https://doi.org/10.1038/nrm3488

Burmester, S. \& Hoyer-Fender, S. (1996). Transcription and translation of the outer dense fiber gene (Odf1) during spermiogenesis in the rat. A study by in situ analyses and polysome fractionation. Molecular Reproduction and Development, 45(1), 10-20. https://doi.org/10.1002/(SICI)1098-2795(199609)45:1<10::AID-MRD2>3.0.CO;2-V

Calvi, A., Wong, A. S. W., Wright, G., Wong, E. S. M., Loo, T. H., Stewart, C. L. \& Burke, B. (2015). SUN4 is essential for nuclear remodeling during mammalian spermiogenesis. Developmental Biology, 407(2), 321-330. https://doi.org/10.1016/j.ydbio.2015.09.010

Chemes, H. E., Carizza, C. \& Scarinci, F. (1987). Lack of a head in human spermatozoa from sterile patients: A syndrome associated with impaired fertilization. International Journal of Gynecology \& Obstetrics, 26(1), 165-165. https://doi.org/10.1016/00207292(88)90230-5

Chemes, H. E., Puigdomenech, E. T., Carizza, C., Olmedo, S. B., Zanchetti, F. \& Hermes, R. (1999). Acephalic spermatozoa and abnormal development of the head-neck attachment: A human syndrome of genetic origin. Human Reproduction, 14(7), 1811-1818. https://doi.org/10.1093/humrep/14.7.1811

Chemes, H. E. (2000). Phenotypes of Sperm Pathology: Genetic and Acquired Forms in Infertile Men. Journal of Andrology, 21(6), 799-808. https://doi.org/10.1002/j.19394640.2000.tb03409.x

Chemes H. E. (2012) Sperm Centrioles and Their Dual Role in Flagellogenesis and Cell Cycle of the Zygote. In: Schatten H. (eds) The Centrosome. Humana Press, Totowa, NJ. https://doi.org/10.1007/978-1-62703-035-9_2 
Chemes, H. E. \& Alvarez Sedo, C. (2012). Tales of the Tail and Sperm Head Aches: Changing concepts on the prognostic significance of sperm pathologies affecting the head, neck and tail. Asian Journal of Andrology, 14(1), 14-23. https://doi.org/10.1038/aja.2011.168

Chemes, H. E. \& Rawe, V. Y. (2003). Sperm pathology: A step beyond descriptive morphology. Origin, characterization and fertility potential of abnormal sperm phenotypes in infertile men. Human Reproduction Update, 9(5), 405-428. https://doi.org/10.1093/humupd/dmg034

Chemes, H. E. \& Rawe, V. Y. (2010). The making of abnormal spermatozoa: Cellular and molecular mechanisms underlying pathological spermiogenesis. Cell and Tissue Research, 341(3), 349-357. https://doi.org/10.1007/s00441-010-1007-3

Chen, H., Zhu, Y., Zhu, Z., Zhi, E., Lu, K., Wang, X., Liu, F., Li, Z. \& Xia, W. (2018). Detection of heterozygous mutation in hook microtubule-tethering protein 1 in three patients with decapitated and decaudated spermatozoa syndrome. Journal of medical genetics, 55(3), 150-157. https://doi.org/10.1136/jmedgenet-2016-104404

Chikashige, Y., Haraguchi, T. \& Hiraoka, Y. (2007). Another way to move chromosomes. Chromosoma, 116(6), 497-505. https://doi.org/10.1007/s00412-007-0114-8

Chin, J. W., Cropp, T. A., Anderson, J. C., Mukherji, M., Zhang, Z. \& Schultz, P. G. (2003). An expanded eukaryotic genetic code. Science (New York, N.Y.), 301(5635), 964-967. https://doi.org/10.1126/science.1084772

Chin, J. W., Santoro, S. W., Martin, A. B., King, D. S., Wang, L. \& Schultz, P. G. (2002). Addition of $p$-Azido- L -phenylalanine to the Genetic Code of Escherichia coli. Journal of the American Chemical Society, 124(31), 9026-9027. https://doi.org/10.1021/ja027007w

Choi, C. P., Moon, A. S., Back, P. S., Jami-Alahmadi, Y., Vashisht, A. A., Wohlschlegel, J. A., Bradley, P. J. (2019). A photoactivatable crosslinking system reveals protein interactions in the Toxoplasma gondii inner membrane complex. PLoS Biology, 17(10): e3000475. https://doi.org/10.1371/journal.pbio.3000475

Clermont, Y. (1972). Kinetics of spermatogenesis in mammals: Seminiferous epithelium cycle and spermatogonial renewal. Physiological Reviews, 52(1), 198-236. https://doi.org/10.1152/physrev.1972.52.1.198

Conrad, M. N., Lee, C. Y., Wilkerson, J. L. \& Dresser, M. E. (2007). MPS3 mediates meiotic bouquet formation in Saccharomyces cerevisiae. Proceedings of the National Academy of Sciences, 104(21), 8863-8868. https://doi.org/10.1073/pnas.0606165104

Costa, Y., Speed, R., Ollinger, R., Alsheimer, M., Semple, C. A., Gautier, P., Maratou, K., Novak, I., Höög, C., Benavente, R. \& Cooke, H. J. (2005). Two novel proteins recruited by synaptonemal complex protein 1 (SYCP1) are at the centre of meiosis. Journal of cell science, 118(Pt 12), 2755-2762. https://doi.org/10.1242/jcs.02402 
Crisp, M., Liu, Q., Roux, K., Rattner, J. B., Shanahan, C., Burke, B., Stahl, P. D. \& Hodzic, D. (2006). Coupling of the nucleus and cytoplasm: Role of the LINC complex. The Journal of Cell Biology, 172(1), 41-53. https://doi.org/10.1083/jcb.200509124

Das, S. \& Oliver, D. B. (2011). Mapping of the SecA·SecY and SecA·SecG interfaces by sitedirected in vivo photocross-linking. The Journal of biological chemistry, 286(14), 1237112380. https://doi.org/10.1074/jbc.M110.182931

De Rooij, D. G. \& Grootegoed, J. A. (1998). Spermatogonial stem cells. Current Opinion in Cell Biology, 10(6), 694-701. https://doi.org/10.1016/s0955-0674(98)80109-9

Dechat, T., Korbei, B., Vaughan, O. A., Vlcek, S., Hutchison, C. J. \& Foisner, R. (2000). Lamina-associated polypeptide 2alpha binds intranuclear A-type lamins. Journal of cell science, 113 Pt 19, 3473-3484.

Dhe-Paganon, S., Werner, E. D., Chi, Y., Shoelson, S. E. (2002). Structure of the Globular Tail of Nuclear Lamin. The Journal of Biological Chemistry, 277, 17381-17384. https://doi.org/ 10.1074/jbc.C200038200

Ding, X., Xu, R., Yu, J., Xu, T., Zhuang, Y. \& Han, M. (2007). SUN1 Is Required for Telomere Attachment to Nuclear Envelope and Gametogenesis in Mice. Developmental Cell, 12(6), 863-872. https://doi.org/10.1016/j.devcel.2007.03.018

Dittmer, T. A. \& Misteli, T. (2011). The lamin protein family. Genome biology, 12(5), 222. https://doi.org/10.1186/gb-2011-12-5-222

Dunleavy, J., O'Bryan, M. K., Stanton, P. G. \& O'Donnell, L. (2019). The cytoskeleton in spermatogenesis. Reproduction (Cambridge, England), 157(2), R53-R72. https://doi.org/10.1530/REP-18-0457

Eddy, E. M. (1998). Regulation of gene expression during spermatogenesis. Seminars in Cell \& Developmental Biology, 9(4), 451-457. https://doi.org/10.1006/scdb.1998.0201

Elkhatib, R. A., Paci, M., Longepied, G., Saias-Magnan, J., Courbiere, B., Guichaoua, M. R., Lévy, N., Metzler-Guillemain, C. \& Mitchell, M. (2017). Homozygous deletion of SUN5 in three men with decapitated spermatozoa. Human Molecular Genetics, 26, 3167-3171. https://doi.org/10.1093/hmg/ddx200

Fawcett, D. W. (1975). The mammalian spermatozoon. Developmental Biology, 44(2), 394436. https://doi.org/10.1016/0012-1606(75)90411-X

Fawcett, D. W. \& Phillips, D. M. (1969). The fine structure and development of the neck region of the mammalian spermatozoon. The Anatomical Record, 165(2), 153-183. https://doi.org/10.1002/ar.1091650204

Fegan, A., White, B., Carlson, J. C. T. \& Wagner, C. R. (2010). Chemically Controlled Protein Assembly: Techniques and Applications. Chemical Reviews, 110(6), 3315-3336. https://doi.org/10.1021/cr8002888 
Fitzgerald, C. J., Oko, R. J. \& van der Hoorn, F. A. (2006). Rat Spag5 associates in somatic cells with endoplasmic reticulum and microtubules but in spermatozoa with outer dense fibers. Molecular Reproduction and Development, 73(1), 92-100. https://doi.org/10.1002/mrd.20388

Fontaine, J. M., Rest, J. S., Welsh, M. J. \& Benndorf, R. (2003). The sperm outer dense fiber protein is the 10th member of the superfamily of mammalian small stress proteins. Cell stress \& chaperones, 8(1), 62-69. https://doi.org/10.1379/1466$1268(2003) 8<62:$ tsodfp $>2.0 . c 0 ; 2$

Frank, C. J., Lorna K. J., Donald P. E. (1986). Mouse Testicular and Sperm Cell Development Characterized from Birth to Adulthood by Dual Parameter Flow Cytometry, Biology of Reproduction, 4(4), 613-623. https://doi.org/10.1095/biolreprod34.4.613

Frohnert, C., Schweizer, S. \& Hoyer-Fender, S. (2011). SPAG4L/SPAG4L-2 are testis-specific SUN domain proteins restricted to the apical nuclear envelope of round spermatids facing the acrosome. Molecular Human Reproduction, 17(4), 207-218. https://doi.org/10.1093/molehr/gaq099

Furukawa, K. \& Hotta, Y. (1993). cDNA cloning of a germ cell specific lamin B3 from mouse spermatocytes and analysis of its function by ectopic expression in somatic cells. The EMBO Journal, 12(1), 97-106. https://doi.org/10.1002/j.1460-2075.1993.tb05635.x

Gerace, L. \& Burke, B. (1988). Functional organization of the nuclear envelope. Annual Review of Cell Biology, 4, 335-374. https://doi.org/10.1146/annurev.cb.04.110188.002003

Gilbert, W. (1978). Why genes in pieces? Nature 271, 501. https://doi.org/10.1038/271501a0

Gilchrist, S., Gilbert, N., Perry, P., Ostlund, C., Worman, H. J., Bickmore, W. A. (2004). Altered protein dynamics of disease-associated lamin A mutants. BMC Cell Biology, 5(1), 46. https://doi.org/10.1186/1471-2121-5-46

Goto, M., O’Brien, D. A. \& Eddy, E. M. (2010). Speriolin is a novel human and mouse sperm centrosome protein. Human Reproduction, 25(8), 1884-1894. https://doi.org/10.1093/humrep/deq138

Göb, E., Schmitt, J., Benavente, R. \& Alsheimer, M. (2010). Mammalian Sperm Head Formation Involves Different Polarization of Two Novel LINC Complexes. PLoS ONE, 5(8), e12072. https://doi.org/10.1371/journal.pone.0012072

Graham, F. L., Smiley, J., Russell, W. C. \& Nairn, R. (1977). Characteristics of a human cell line transformed by DNA from human adenovirus type 5. The Journal of General Virology, 36(1), 59-74. https://doi.org/10.1099/0022-1317-36-1-59

Hackstein, J. H., Hochstenbach, R. \& Pearson, P. L. (2000). Towards an understanding of the genetics of human male infertility: lessons from flies. Trends in genetics: TIG, 16(12), 565-572. https://doi.org/10.1016/s0168-9525(00)02140-5 
Hagan, I. \& Yanagida, M. (1995). The product of the spindle formation gene sad1+ associates with the fission yeast spindle pole body and is essential for viability. The Journal of Cell Biology, 129(4), 1033-1047. https://doi.org/10.1083/jcb.129.4.1033

Haque, F., Lloyd, D. J., Smallwood, D. T., Dent, C. L., Shanahan, C. M., Fry, A. M., Trembath, R. C., \& Shackleton, S. (2006). SUN1 Interacts with Nuclear Lamin A and Cytoplasmic Nesprins To Provide a Physical Connection between the Nuclear Lamina and the Cytoskeleton. Molecular and Cellular Biology, 26(10), 3738-3751. https://doi.org/10.1128/MCB.26.10.3738-3751.2006

Hasan, S., Güttinger, S., Mühlhäusser, P., Anderegg, F., Bürgler, S. \& Kutay, U. (2006). Nuclear envelope localization of human UNC84A does not require nuclear lamins. FEBS letters, 580(5), 1263-1268. https://doi.org/10.1016/j.febslet.2006.01.039

Hermo, L., Pelletier, R.-M., Cyr, D. G., \& Smith, C. E. (2009b). Surfing the wave, cycle, life history, and genes/proteins expressed by testicular germ cells. Part 3: Developmental changes in spermatid flagellum and cytoplasmic droplet and interaction of sperm with the zona pellucida and egg plasma membrane. Microscopy Research and Technique, 73(4), 320-363. https://doi.org/10.1002/jemt.20784

Hermo, L., Pelletier, R. M., Cyr, D. G. \& Smith, C. E. (2010). Surfing the wave, cycle, life history, and genes/proteins expressed by testicular germ cells. Part 1: Background to spermatogenesis, spermatogonia, and spermatocytes. Microscopy Research and Technique, 73(4), 241-278. https://doi.org/10.1002/jemt.20783

Hinegardner, R. T., Engelberg, J. (1963). Rationale for a universal genetic code. Science, 142(3595), 1083-1085. https://doi.org/10.1126/science.142.3595.1083

Hino, N., Okazaki, Y., Kobayashi, T., Hayashi, A., Sakamoto, K. \& Yokoyama, S. (2005). Protein photo-cross-linking in mammalian cells by site-specific incorporation of a photoreactive amino acid. Nature Methods, 2(3), 201-206. https://doi.org/10.1038/nmeth739

Hodzic, D. M., Yeater, D. B., Bengtsson, L., Otto, H. \& Stahl, P. D. (2004). Sun2 Is a Novel Mammalian Inner Nuclear Membrane Protein. Journal of Biological Chemistry, 279(24), 25805-25812. https://doi.org/10.1074/jbc.M313157200

Hofferbert, S., Burfeind, P., Hoyer-Fender, S., Lange, R., Haldl, G. \& Engel, W. (1993). A homozygous deletion of 27 base pairs in the coding region of the human outer dense fiber protein gene does not result in a pathologic phenotype. Human Molecular Genetics, 2(12), 2167-2170. https://doi.org/10.1093/hmg/2.12.2167

Horvitz, H. R. \& Sulston, J. E. (1980). Isolation and genetic characterization of cell-lineage mutants of the nematode Caenorhabditis elegans. Genetics, 96(2), 435-454.

Hoyer-Fender, S., Burfeind, P. \& Hameister, H. (1995). Sequence of mouse Odf1 cDNA and its chromosomal localization: Extension of the linkage group between human 
chromosome 8 and mouse chromosome 15. Cytogenetics and Cell Genetics, 70(3-4), 200-204. https://doi.org/10.1159/000134033

Hoyer-Fender, S. (2010). Centriole maturation and transformation to basal body. Seminars in Cell \& Developmental Biology, 21(2), 142-147. https://doi.org/10.1016/j.semcdb.2009.07.002

Hoyer-Fender, S. (2012). Centrosomes in fertilization, early embryonic development, stem cell division, and cancer. Atlas of Genetics and Cytogenetics in Oncology and Haematology, 16(4):306-319. https://doi.org/10.4267/2042/47311

Inobe, T. \& Nukina, N. (2016). Rapamycin-induced oligomer formation system of FRB-FKBP fusion proteins. Journal of Bioscience and Bioengineering, 122(1), 40-46. https://doi.org/10.1016/j.jbiosc.2015.12.004

Ito, C., Akutsu, H., Yao, R., Yoshida, K., Yamatoya, K., Mutoh, T., Makino, T., Aoyama, K., Ishikawa, H., Kunimoto, K., Tsukita, S., Noda, T., Kikkawa, M. \& Toshimori, K. (2019). Odf2 haploinsufficiency causes a new type of decapitated and decaudated spermatozoa, Odf2-DDS, in mice. Scientific Reports, 9(1), 14249. https://doi.org/10.1038/s41598-019-50516-2

Iwashita, T. \& Oura, C. (1980). A Three Dimensional Analysis of the Capitellum and Striated Columns in the Sperm Neck Region of the Mouse. Okajimas Folia Anatomica Japonica, 56(6), 361-381. https://doi.org/10.2535/ofaj1936.56.6_361

Kamal, A., Mansour, R., Fahmy, I., Serour, G., Rhodes, C. \& Aboulghar, M. (1999). Easily decapitated spermatozoa defect: a possible cause of unexplained infertility. Human $\begin{array}{llll}\text { reproduction } \quad \text { (Oxford, } & \text { England), }\end{array}$ https://doi.org/10.1093/humrep/14.11.2791

Kauer, J. C., Erickson-Viitanen, S., Wolfe, H. R. \& DeGrado, W. F. (1986). p-Benzoyl-Lphenylalanine, a new photoreactive amino acid. Photolabeling of calmodulin with a synthetic calmodulin-binding peptide. The Journal of biological chemistry, 261(23), 10695-10700.

Kellogg, D. R., Moritz, M. \& Alberts, B. M. (1994). The Centrosome and Cellular Organization. Annual Review of Biochemistry, 63(1), 639-674. https://doi.org/10.1146/annurev.bi.63.070194.003231

Kelly, S., Georgomanolis, T., Zirkel, A., Diermeier, S., O'Reilly, D., Murphy, S., Längst, G., Cook, P. R. \& Papantonis, A. (2015). Splicing of many human genes involves sites embedded within introns. Nucleic acids research, 43(9), 4721-4732. https://doi.org/10.1093/nar/gkv386

Kierszenbaum, A. L., Rivkin, E. \& Tres, L. L. (2003). Acroplaxome, an F-Actin-Keratincontaining Plate, Anchors the Acrosome to the Nucleus during Shaping of the Spermatid 
Head. Molecular Biology of the Cell, 14(11), 4628-4640. https://doi.org/10.1091/mbc.e03-04-0226

Kierszenbaum, A. L., Rivkin, E., Tres, L. L., Yoder, B. K., Haycraft, C. J., Bornens, M. \& Rios, R. M. (2011). GMAP210 and IFT88 are present in the spermatid golgi apparatus and participate in the development of the acrosome-acroplaxome complex, head-tail coupling apparatus and tail. Developmental Dynamics, 240(3), 723-736. https://doi.org/10.1002/dvdy.22563

Kierszenbaum, A. L. \& Tres, L. L. (2002). Bypassing natural sperm selection during fertilization: The azh mutant offspring experience and the alternative of spermiogenesis in vitro. Molecular and Cellular Endocrinology, 187(1-2), 133-138. https://doi.org/10.1016/S0303-7207(01)00692-X

Kierszenbaum, A. L. \& Tres, L. L. (2004). The acrosome-acroplaxome-manchette complex and the shaping of the spermatid head. Archives of Histology and Cytology, 67(4), 271-284. https://doi.org/10.1679/aohc.67.271

Kierszenbaum, A. L. \& Tres, L. L. (2016). Histology and Cell Biology: An Introduction to Pathology - 4th Edition. Philadelphia, PA: Elsevier Saunders.

Kite, G. L. (1913). The Relative Permeability of the Surface and Interior Portions of the Cytoplasm of Animal and Plant Cells. (A Preliminary Paper). Biological Bulletin, 25(1), 1. https://doi.org/10.2307/1536080

Knight, R., Freeland, S. \& Landweber, L. (2001). Rewiring the keyboard: evolvability of the genetic code. Nature Reviews. Genetics, 2(1), 49-58. https://doi.org/10.1038/35047500

Kochanski, R. S. \& Borisy, G. G. (1990). Mode of centriole duplication and distribution. The Journal of Cell Biology, 110(5), 1599-1605. https://doi.org/10.1083/jcb.110.5.1599

Kracklauer, M. P., Wiora, H. M., Deery, W. J., Chen, X., Bolival, B., Romanowicz, D., Simonette, R. A., Fuller, M. T., Fischer, J. A. \& Beckingham, K. M. (2010). The Drosophila SUN protein Spag4 cooperates with the coiled-coil protein Yuri Gagarin to maintain association of the basal body and spermatid nucleus. Journal of Cell Science, 123(Pt 16), 2763-2772. https://doi.org/10.1242/jcs.066589

Krimm, I., Ostlund, C., Gilquin, B., Couprie, J., Hossenlopp, P., Mornon, J. P., Bonne, G., Courvalin, J. C., Worman, H. J. \& Zinn-Justin, S. (2002). The Ig-like structure of the Cterminal domain of lamin $\mathrm{A} / \mathrm{C}$, mutated in muscular dystrophies, cardiomyopathy, and partial lipodystrophy. Structure (London, England: 1993), 10(6), 811-823. https://doi.org/10.1016/s0969-2126(02)00777-3

Lee, C. \& Chen, L. B. (1988). Dynamic behavior of endoplasmic reticulum in living cells. Cell, 54(1), 37-46. https://doi.org/10.1016/0092-8674(88)90177-8 
Lehti, M. S., Kotaja, N. \& Sironen, A. (2013). KIF3A is essential for sperm tail formation and manchette function. Molecular and Cellular Endocrinology, 377(1-2), 44-55. https://doi.org/10.1016/j.mce.2013.06.030

Li, D. \& Roberts, R. (2001). WD-repeat proteins: structure characteristics, biological function, and their involvement in human diseases. Cellular and molecular life sciences : CMLS, 58(14), 2085-2097. https://doi.org/10.1007/p100000838

Lindemann, C. B. (1996). Functional significance of the outer dense fibers of mammalian sperm examined by computer simulations with the geometric clutch model. Cell Motility and the Cytoskeleton, 34(4), 258-270.

https://doi.org/10.1002/(SICI)1097-0169(1996)34:4<258::AID-CM1>3.0.CO;2-4

Liška, F., Gosele, C., Popova, E., Chylíková, B., Křenová, D., Křen, V., Bader, M., Tres, L. L., Hubner, N. \& Kierszenbaum, A. L. (2013). Overexpression of Full-Length Centrobin Rescues Limb Malformation but Not Male Fertility of the Hypodactylous (hd) Rats. PLoS ONE, 8(4), e60859. https://doi.org/10.1371/journal.pone.0060859

Liška, F., Gosele, C., Rivkin, E., Tres, L., Cardoso, M. C., Domaing, P., Krejčí, E., Šnajdr, P., Lee-Kirsch, M. A., Rooij, D. G. de, Křen, V., Křenová, D., Kierszenbaum, A. L. \& Hubner, N. (2009). Rat $h d$ Mutation Reveals an Essential Role of Centrobin in Spermatid Head Shaping and Assembly of the Head-Tail Coupling Apparatus1. Biology of Reproduction, 81(6), 1196-1205. https://doi.org/10.1095/biolreprod.109.078980

Liu, W., Brock, A., Chen, S., Chen, S. \& Schultz, P. G. (2007). Genetic incorporation of unnatural amino acids into proteins in mammalian cells. Nature Methods, 4(3), 239-244. https://doi.org/10.1038/nmeth1016

Longenecker, G. \& Kulkarni, A. B. (2009). Generation of gene knockout mice by ES cell microinjection. Current protocols in cell biology, Chapter 19, Unit-19.14.36. https://doi.org/10.1002/0471143030.cb1914s44

Łuksza, M., Queguigner, I., Verlhac, M.-H. \& Brunet, S. (2013). Rebuilding MTOCs upon centriole loss during mouse oogenesis. Developmental Biology, 382(1), 48-56. https://doi.org/10.1016/j.ydbio.2013.07.029

Ma, H. T., Niu, C. M., Xia, J., Shen, X. Y., Xia, M. M., Hu, Y. Q. \& Zheng, Y. (2018). Stimulated by retinoic acid gene 8 (Stra8) plays important roles in many stages of spermatogenesis. Asian journal of andrology, 20(5), 479-487. https://doi.org/10.4103/aja.aja_26_18

MacKinnon, E. A., Abraham, P. J. \& Svatek, A. (1973). Long link induction between the microtubules of the manchette in intermediate stages of spermiogenesis. Zeitschrift Für Zellforschung Und Mikroskopische Anatomie (Vienna, Austria: 1948), 136(4), 447-460. https://doi.org/10.1007/BF00307363 
Malone, C. J., Fixsen, W. D., Horvitz, H. R. \& Han, M. (1999). UNC-84 localizes to the nuclear envelope and is required for nuclear migration and anchoring during C. elegans development. Development, 126(14), 3171-3181. https://dev.biologists.org/content/126/14/3171

Malone, C. J., Misner, L., Le Bot, N., Tsai, M.-C., Campbell, J. M., Ahringer, J. \& White, J. G. (2003). The C. elegans Hook Protein, ZYG-12, Mediates the Essential Attachment between the Centrosome and Nucleus. Cell, 115(7), 825-836. https://doi.org/10.1016/S0092-8674(03)00985-1

Manandhar, G. \& Schatten, G. (2000). Centrosome reduction during Rhesus spermiogenesis: Gamma-tubulin, centrin, and centriole degeneration. Molecular Reproduction and Development, 56(4), 502-511. https://doi.org/10.1002/1098-2795(200008)56:4<502::AID-MRD8>3.0.CO;2-Q

Manandhar, G., Sutovsky, P., Joshi, H. C., Stearns, T. \& Schatten, G. (1998). Centrosome Reduction during Mouse Spermiogenesis. Developmental Biology, 203(2), 424-434. https://doi.org/10.1006/dbio.1998.8947

Martin, G. R. (1981). Isolation of a pluripotent cell line from early mouse embryos cultured in medium conditioned by teratocarcinoma stem cells. Proceedings of the National Academy of Sciences, 78(12), 7634-7638. https://doi.org/10.1073/pnas.78.12.7634

Matzuk, M. M. \& Lamb, D. J. (2002). Genetic dissection of mammalian fertility pathways. Nature cell biology, 4 Suppl, s41-s49. https://doi.org/10.1038/ncb-nm-fertilityS41

Mendoza-Lujambio, I. (2002). The Hook1 gene is non-functional in the abnormal spermatozoon head shape (azh) mutant mouse. Human Molecular Genetics, 11(14), 1647-1658. https://doi.org/10.1093/hmg/11.14.1647

Meuwissen, R. L., Offenberg, H. H., Dietrich, A. J., Riesewijk, A., van Iersel, M. \& Heyting, C. (1992). A coiled-coil related protein specific for synapsed regions of meiotic prophase chromosomes. The EMBO journal, 11(13), 5091-5100.

Minn, I., Rolls, M. M., Hanna-Rose, W. \& Malone, C. J. (2009). SUN-1 and ZYG-12, Mediators of Centrosome-Nucleus Attachment, Are a Functional SUN/KASH Pair in Caenorhabditis elegans. Molecular Biology of the Cell, 20(21), 4586-4595. https://doi.org/10.1091/mbc.E08-10-1034

Miyamoto, T., Tsujimura, A., Miyagawa, Y., Koh, E., Namiki, M. \& Sengoku, K. (2012). Male Infertility and Its Causes in Human. Advances in Urology, 2012, 1-7. https://doi.org/10.1155/2012/384520

Morales, C. R., Oko, R. \& Clermont, Y. (1994). Molecular cloning and developmental expression of an mRNA encoding the $27 \mathrm{kDa}$ outer dense fiber protein of rat spermatozoa. Molecular Reproduction and Development, 37(2), 229-240. https://doi.org/10.1002/mrd.1080370215 
Nebel, B. R., Amarose, A. P. \& Hacket, E. M. (1961). Calendar of gametogenic development in the prepuberal male mouse. Science, 134, 832-833. https://doi.org/10.1126/science.134.3482.832

Neer, E. J., Schmidt, C. J., Nambudripad, R. \& Smith, T. F. (1994). The ancient regulatoryprotein family of WD-repeat proteins. Nature, 371(6495), 297-300. https://doi.org/10.1038/371297a0

Netzel-Arnett, S., Bugge, T. H., Hess, R. A., Carnes, K., Stringer, B. W., Scarman, A. L., Hooper, J. D., Tonks, I. D., Kay, G. F. \& Antalis, T. M. (2009). The Glycosylphosphatidylinositol-Anchored Serine Protease PRSS21 (Testisin) Imparts Murine Epididymal Sperm Cell Maturation and Fertilizing Ability1. Biology of Reproduction, 81(5), 921-932. https://doi.org/10.1095/biolreprod.109.076273

Nozawa, Y. I., Yao, E., Gacayan, R., Xu, S.-M. \& Chuang, P.-T. (2014). Mammalian Fused is essential for sperm head shaping and periaxonemal structure formation during spermatogenesis. Developmental Biology, 388(2), 170-180. https://doi.org/10.1016/j.ydbio.2014.02.002

Oakberg, E. F. (1956). A description of spermiogenesis in the mouse and its use in analysis of the cycle of the seminiferous epithelium and germ cell renewal. American Journal of Anatomy, 99(3), 391-413. https://doi.org/10.1002/aja.1000990303

O’Donnell, L., \& O'Bryan, M. K. (2014). Microtubules and spermatogenesis. Seminars in Cell \& Developmental Biology, 30, 45-54. https://doi.org/10.1016/j.semcdb.2014.01.003

Oko, R. (1988). Comparative Analysis of Proteins from the Fibrous Sheath and Outer Dense Fibers of Rat Spermatozoa1. Biology of Reproduction, 39(1), 169-182. https://doi.org/10.1095/biolreprod39.1.169

Osawa, S., Jukes, T. H., Watanabe, K. \& Muto, A. (1992). Recent evidence for evolution of the genetic code. Microbiological reviews, 56(1), 229-264. https://doi.org/10.1128/MMBR.56.1.229-264.1992

Padmakumar, V. C. (2005). The inner nuclear membrane protein Sun1 mediates the anchorage of Nesprin-2 to the nuclear envelope. Journal of Cell Science, 118(15), 3419-3430. https://doi.org/10.1242/jcs.02471

Paine, P. L. \& Feldherr, C. M. (1972). Nucleocytoplasmic exchange of macromolecules. Experimental cell research, 74(1), 81-98. https://doi.org/10.1016/0014-4827(72)90483-1

Palmiter, R. D., Chen, H. Y. \& Brinster, R. L. (1982). Differential regulation of metallothioneinthymidine kinase fusion genes in transgenic mice and their offspring. Cell, 29(2), 701710. https://doi.org/10.1016/0092-8674(82)90186-6 
Pasch, E., Link, J., Beck, C., Scheuerle, S. \& Alsheimer, M. (2015). The LINC complex component Sun4 plays a crucial role in sperm head formation and fertility. Biology Open, 4(12), 1792-1802. https://doi.org/10.1242/bio.015768

Pasek, R. C., Malarkey, E., Berbari, N. F., Sharma, N., Kesterson, R. A., Tres, L. L., Kierszenbaum, A. L. \& Yoder, B. K. (2016). Coiled-coil domain containing 42 (Ccdc42) is necessary for proper sperm development and male fertility in the mouse. Developmental Biology, 412(2), 208-218. https://doi.org/10.1016/j.ydbio.2016.01.042

Perotti, M. E., Giarola, A. \& Gioria, M. (1981). Ultrastructural study of the decapitated sperm defect in an infertile man. Journal of Reproduction and Fertility, 63(2), 543-549. https://doi.org/10.1530/jrf.0.0630543

Petersen, C. (1999). Outer dense fibre proteins from human sperm tail: Molecular cloning and expression analyses of two cDNA transcripts encoding proteins of $70 \mathrm{kDa}$. Molecular Human Reproduction, 5(7), 627-635. https://doi.org/10.1093/molehr/5.7.627

Petersen, C., Aumüller, G., Bahrami, M. \& Hoyer-Fender, S. (2002). Molecular cloning of Odf3 encoding a novel coiled-coil protein of sperm tail outer dense fibers. Molecular Reproduction and Development, 61(1), 102-112. https://doi.org/10.1002/mrd.1136

Qi, Y., Jiang, M., Yuan, Y., Bi, Y., Zheng, B., Guo, X., Huang, X., Zhou, Z. \& Sha, J. (2013). ADP-ribosylation factor-like 3, a manchette-associated protein, is essential for mouse spermiogenesis. Molecular Human Reproduction, 19(5), 327-335. https://doi.org/10.1093/molehr/gat001

Rattner, J. B. \& Olson, G. (1973). Observations on the fine structure of the nuclear ring of the mammalian spermatid. Journal of Ultrastructure Research, 43(5), 438-444. https://doi.org/10.1016/S0022-5320(73)90020-8

Razafsky, D. \& Hodzic, D. (2009). Bringing KASH under the SUN: The many faces of nucleocytoskeletal connections. The Journal of Cell Biology, 186(4), 461-472. https://doi.org/10.1083/jcb.200906068

Rober, R. A., Weber, K. \& Osborn, M. (1989). Differential timing of nuclear lamin A/C expression in the various organs of the mouse embryo and the young animal: A $\begin{array}{llll}\text { developmental } & \text { study. } & \text { Development, } & 105(2),\end{array}$ http://dev.biologists.org/content/105/2/365.abstract

Russell, L. D. (1990). Histological and histopathological evaluation of the testis. Clearwater, Fla.: Cache River Press. http://agris.fao.org/agris-search/search.do?recordID=US201300697285

Salpingidou, G., Smertenko, A., Hausmanowa-Petrucewicz, I., Hussey, P. J. \& Hutchison, C. J. (2007). A novel role for the nuclear membrane protein emerin in association of the centrosome to the outer nuclear membrane. The Journal of Cell Biology, 178(6), 897-904. https://doi.org/10.1083/jcb.200702026 
Schalles, U., Shao, X., van der Hoorn, F. A. \& Oko, R. (1998). Developmental Expression of the 84-kDa ODF Sperm Protein: Localization to both the Cortex and Medulla of Outer Dense Fibers and to the Connecting Piece. Developmental Biology, 199(2), 250-260. https://doi.org/10.1006/dbio.1998.8931

Schatten, H., Schatten, G., Mazia, D., Balczon, R. \& Simerly, C. (1986). Behavior of centrosomes during fertilization and cell division in mouse oocytes and in sea urchin eggs. Proceedings of the National Academy of Sciences, 83(1), 105-109. https://doi.org/10.1073/pnas.83.1.105

Schatten, H., \& Sun, Q. Y. (2009). The role of centrosomes in mammalian fertilization and its significance for ICSI. Molecular Human Reproduction, 15(9), 531-538. https://doi.org/10.1093/molehr/gap049

Schirmer, E. C., Florens, L., Guan, T., Yates, J. R. \& Gerace, L. (2003). Nuclear Membrane Proteins with Potential Disease Links Found by Subtractive Proteomics. Science, 301(5638), 1380-1382. https://doi.org/10.1126/science.1088176

Schmitt, J., Benavente, R., Hodzic, D., Höög, C., Stewart, C. L. \& Alsheimer, M. (2007). Transmembrane protein Sun2 is involved in tethering mammalian meiotic telomeres to the nuclear envelope. Proceedings of the National Academy of Sciences of the United States of America, 104(18), 7426-7431. https://doi.org/10.1073/pnas.0609198104

Schütz, W., Alsheimer, M., Öllinger, R. \& Benavente, R. (2005). Nuclear envelope remodeling during mouse spermiogenesis: Postmeiotic expression and redistribution of germline lamin B3. Experimental Cell Research, 307(2), 285-291. https://doi.org/10.1016/j.yexcr.2005.03.023

Schweizer, S. \& Hoyer-Fender, S. (2009). Mouse Odf2 localizes to centrosomes and basal bodies in adult tissues and to the photoreceptor primary cilium. Cell and Tissue Research 338, 295. https://doi.org/10.1007/s00441-009-0861-3

Sha, Y. W., Xu, X., Ji, Z. Y., Lin, S. B., Wang, X., Qiu, P. P., Zhou, Y., Mei, L. B., Su, Z. Y., Li, L. \& Li, P. (2018). Genetic contribution of SUN5 mutations to acephalic spermatozoa in Fujian China. Gene, 647, 221-225. https://doi.org/10.1016/j.gene.2018.01.035

Sha, Y. W., Wang, X., Xu, X., Ding, L., Liu, W. S., Li, P., Su, Z. Y., Chen, J., Mei, L. B., Zheng, L. K., Wang, H. L., Kong, S. B., You, M. \& Wu, J. F. (2019). Biallelic mutations in PMFBP1 cause acephalic spermatozoa. Clinical genetics, 95(2), 277-286. https://doi.org/10.1111/cge.13461

Shang, Y., Yan, J., Tang, W., Liu, C., Xiao, S., Guo, Y., Yuan, L., Chen, L., Jiang, H., Guo, X., Qiao, J. \& Li, W. (2018). Mechanistic insights into acephalic spermatozoa syndromeassociated mutations in the human SUN5 gene. The Journal of Biological Chemistry, 293(7), 2395-2407. https://doi.org/10.1074/jbc.RA117.000861 
Shang, Y., Zhu, F., Wang, L., Ouyang, Y.-C., Dong, M.-Z., Liu, C., Zhao, H., Cui, X., Ma, D., Zhang, Z., Yang, X., Guo, Y., Liu, F., Yuan, L., Gao, F., Guo, X., Sun, Q.-Y., Cao, Y. \& Li, W. (2017). Essential role for SUN5 in anchoring sperm head to the tail. ELife, 6, e28199. https://doi.org/10.7554/eLife.28199

Shao, X., Tarnasky, H. A., Lee, J. P., Oko, R. \& van der Hoorn, F. A. (1999). Spag4, a Novel Sperm Protein, Binds Outer Dense-Fiber Protein Odf1 and Localizes to Microtubules of Manchette and Axoneme. Developmental Biology, 211(1), 109-123. https://doi.org/10.1006/dbio.1999.9297

Shao, X., Tarnasky, H. A., Schalles, U., Oko, R. \& van der Hoorn, F. A. (1997). Interactional Cloning of the 84-kDa Major Outer Dense Fiber Protein Odf84: leucine zippers mediate associations of Odf84 and Odf27. Journal of Biological Chemistry, 272(10), 6105-6113. https://doi.org/10.1074/jbc.272.10.6105

Shao, X. \& van der Hoorn, F. A. (1996). Self-Interaction of the Major 27-Kilodalton Outer Dense Fiber Protein is in Part Mediated by a Leucine Zipper Domain in the Rat1. Biology of Reproduction, 55(6), 1343-1350. https://doi.org/10.1095/biolreprod55.6.1343

Shao, X., Xue, J. \& van der Hoorn, F. A. (2001). Testicular protein Spag5 has similarity to mitotic spindle protein Deepest and binds outer dense fiber protein Odf1. Molecular Reproduction and Development, 59(4), 410-416. https://doi.org/10.1002/mrd.1047

Sharlip, I. D., Jarow, J. P., Belker, A. M., Lipshultz, L. I., Sigman, M., Thomas, A. J., Schlegel, P. N., Howards, S. S., Nehra, A., Damewood, M. D., Overstreet, J. W. \& Sadovsky, R. (2002). Best practice policies for male infertility. Fertility and Sterility, 77(5), 873-882. https://doi.org/10.1016/s0015-0282(02)03105-9

Sharma, A. (2017). Male Infertility: Evidences, Risk Factors, Causes, Diagnosis and Management in Human. Annals of Clinical and Laboratory Research, 05(03). https://doi.org/10.21767/2386-5180.1000188

Sharp, P. M. \& Bulmer, M. (1988). Selective differences among translation termination codons. Gene, 63 (1), 141-145. https://doi.org/10.1016/0378-1119(88)90553-7

Simerly, C., Wu, G.-J., Zoran, S., Ord, T., Rawlins, R., Jones, J., Navara, C., Gerrity, M., Rinehart, J., Binor, Z., Asch, R. \& Schatten, G. (1995). The paternal inheritance of the centrosome, the cell's microtubule-organizing center, in humans, and the implications for infertility. Nature Medicine, 1(1), 47-52. https://doi.org/10.1038/nm0195-47

Starr, D. A. (2002). Role of ANC-1 in Tethering Nuclei to the Actin Cytoskeleton. Science, 298(5592), 406-409. https://doi.org/10.1126/science.1075119

Starr, D. A. (2003). ANChors away: An actin based mechanism of nuclear positioning. Journal of Cell Science, 116(2), 211-216. https://doi.org/10.1242/jcs.00248 
Starr, D. A. \& Fridolfsson, H. N. (2010). Interactions Between Nuclei and the Cytoskeleton Are Mediated by SUN-KASH Nuclear-Envelope Bridges. Annual Review of Cell and Developmental Biology, 26(1), 421-444. https://doi.org/10.1146/annurev-cellbio100109-104037

Starr, D. A. \& Han, M. (2003). ANChors away: An actin based mechanism of nuclear positioning. Journal of Cell Science, 116(2), 211. https://doi.org/10.1242/jcs.00248

Tanaka, H., Iguchi, N., Isotani, A., Kitamura, K., Toyama, Y., Matsuoka, Y., Onishi, M., Masai, K., Maekawa, M., Toshimori, K., Okabe, M. \& Nishimune, Y. (2005). HANP1/H1T2, a Novel Histone H1-Like Protein Involved in Nuclear Formation and Sperm Fertility. Molecular and Cellular Biology, 25(16), 7107-7119. https://doi.org/10.1128/MCB.25.16.7107-7119.2005

Tapia Contreras, C. \& Hoyer-Fender, S. (2019). CCDC42 Localizes to Manchette, HTCA and Tail and Interacts With ODF1 and ODF2 in the Formation of the Male Germ Cell Cytoskeleton. Frontiers in Cell and Developmental Biology, 7, 151. https://doi.org/10.3389/fcell.2019.00151

Todaro, G. J. \& Green, H. (1963). Quantitative studies of the growth of mouse embryo cells in culture and their development into established lines. The Journal of Cell Biology, 17, 299-313. https://doi.org/10.1083/jcb.17.2.299

Tokuhiro, K., Isotani, A., Yokota, S., Yano, Y., Oshio, S., Hirose, M., Wada, M., Fujita, K., Ogawa, Y., Okabe, M., Nishimune, Y. \& Tanaka, H. (2009). OAZ-t/OAZ3 Is Essential for Rigid Connection of Sperm Tails to Heads in Mouse. PLoS Genetics, 5(11), e1000712. https://doi.org/10.1371/journal.pgen.1000712

Toyama, Y., Iwamoto, T., Yajima, M., Baba, K. \& Yuasa, S. (2000). Decapitated and decaudated spermatozoa in man, and pathogenesis based on the ultrastructure. International Journal of Andrology, 23(2), 109-115. https://doi.org/10.1046/j.1365-2605.2000.t01-1-00217.x

Tzur, Y. B., Wilson, K. L. \& Gruenbaum, Y. (2006). SUN-domain proteins: "Velcro" that links the nucleoskeleton to the cytoskeleton. Nature Reviews Molecular Cell Biology, 7(10), 782-788. https://doi.org/10.1038/nrm2003

Urbani, L \& Stearns, T. (1999). The centrosome. Current Biology: CB, 9(9), R315-317. https://doi.org/10.1016/s0960-9822(99)80201-2

Van der Hoorn, F. A., Tarnasky, H. A. \& Nordeen, S. K. (1990). A new rat gene RT7 is specifically expressed during spermatogenesis. Developmental Biology, 142(1), 147-154. https://doi.org/10.1016/0012-1606(90)90158-F

Vera, J. C., Brito, M., Zuvic, T. \& Burzio, L. O. (1984). Polypeptide composition of rat sperm outer dense fibers. A simple procedure to isolate the fibrillar complex. The Journal of Biological Chemistry, 259(9), 5970-5977. 
Villasante, A., Wang, D., Dobner, P., Dolph, P., Lewis, S. A. \& Cowan, N. J. (1986). Six mouse alpha-tubulin mRNAs encode five distinct isotypes: testis-specific expression of two sister genes. Molecular and cellular biology, 6(7), 2409-2419. https://doi.org/10.1128/mcb.6.7.2409

Vogt, P. H. (2004). Molecular genetics of human male infertility: from genes to new therapeutic perspectives. Current pharmaceutical design, 10(5), 471-500. https://doi.org/10.2174/1381612043453261

Vorobjev, I. A. \& Chentsov, Y. S. (1980). The ultrastructure of centriole in mammalian tissue culture cells. Cell Biology International Reports, 4(11), 1037-1044. https://doi.org/10.1016/0309-1651(80)90177-0

Vorobjev, I. A., \& Chentsov, Y. S. (1982). Centrioles in the cell cycle. I. Epithelial cells. The Journal of Cell Biology, 93(3), 938-949. https://doi.org/10.1083/jcb.93.3.938

Walenta, J. H., Didier, A. J., Liu, X. \& Krämer, H. (2001). The Golgi-Associated Hook3 Protein Is a Member of a Novel Family of Microtubule-Binding Proteins. The Journal of Cell Biology, 152(5), 923-934. https://doi.org/10.1083/jcb.152.5.923

Wang, Q., Du, X., Cai, Z. \& Greene, M. I. (2006). Characterization of the Structures Involved in Localization of the SUN Proteins to the Nuclear Envelope and the Centrosome. DNA and Cell Biology, 25(10), 554-562. https://doi.org/10.1089/dna.2006.25.554

Watson, M. L. (1955). The nuclear envelope. The Journal of Biophysical and Biochemical Cytology, 1(3), 257-270. https://www.ncbi.nlm.nih.gov/pmc/articles/PMC2223813/

Wilhelmsen, K., Ketema, M., Truong, H. \& Sonnenberg, A. (2006). KASH-domain proteins in nuclear migration, anchorage and other processes. Journal of Cell Science, 119(24), 50215029. https://doi.org/10.1242/jcs.03295

Wilkie, T. M., Brinster, R. L. \& Palmiter, R. D. (1986). Germline and somatic mosaicism in transgenic mice. Developmental Biology, 118(1), 9-18. https://doi.org/10.1016/0012-1606(86)90068-0

Woese, C. R, Hinegardner, R. T., Engelberg, J. (1964). Universality in the Genetic Code. Science, 144(3621), 1030-1031. https://doi.org/10.1126/science.144.3621.1030

World Health Organization. (2010). WHO laboratory manual for the examination and processing of human semen. (5th ed). World Health Organization. https://apps.who.int/iris/handle/10665/44261

Xie, J. \& Schultz, P. G. (2005). An expanding genetic code. Methods, 36(3), 227-238. https://doi.org/10.1016/j.ymeth.2005.04.010 
Xing, X. W., Li, L.-Y., Liu, G., Fu, J.-J., Tan, X.-J. \& Lu, G.-X. (2004). Identification of a Novel Gene SRG4 Expressed at Specific Stages of Mouse Spermatogenesis. Acta Biochimica et Biophysica Sinica, 36(5), 351-359. https://doi.org/10.1093/abbs/36.5.351

Yang, K., Meinhardt, A., Zhang, B., Grzmil, P., Adham, I. M. \& Hoyer-Fender, S. (2012). The Small Heat Shock Protein ODF1/HSPB10 Is Essential for Tight Linkage of Sperm Head to Tail and Male Fertility in Mice. Molecular and Cellular Biology, 32(1), 216-225. https://doi.org/10.1128/MCB.06158-11

Yang, K., Adham, I. M., Meinhardt, A., \& Hoyer-Fender, S. (2018). Ultra-structure of the sperm head-to-tail linkage complex in the absence of the spermatid-specific LINC component SPAG4. Histochemistry and Cell Biology, 150(1), 49-59. https://doi.org/10.1007/s00418-018-1668-7

Yang, K., Grzmil, P., Meinhardt, A., \& Hoyer-Fender, S. (2014). Haplo-deficiency of ODF1/HSPB10 in mouse sperm causes relaxation of head-to-tail linkage. Reproduction, 148(5), 499-506. https://doi.org/10.1530/REP-14-0370

Yassine, S., Escoffier, J., Nahed, R. A., Pierre, V., Karaouzene, T., Ray, P. F. \& Arnoult, C. (2015). Dynamics of Sun5 Localization during Spermatogenesis in Wild Type and Dpy 1912 Knock-Out Mice Indicates That Sun5 Is Not Involved in Acrosome Attachment to the Nuclear Envelope. PloS one, 10(3), e0118698. https://doi.org/10.1371/journal.pone.0118698

Ye, Q. \& Worman, H. J. (1995). Protein-protein interactions between human nuclear lamins expressed in yeast. Experimental Cell Research, 219(1), 292-298. https://doi.org/10.1006/excr.1995.1230

Yuan, L., Liu, J. G., Zhao, J., Brundell, E., Daneholt, B. \& Höög, C. (2000). The murine SCP3 gene is required for synaptonemal complex assembly, chromosome synapsis, and male fertility. Molecular cell, 5(1), 73-83. https://doi.org/10.1016/s1097-2765(00)80404-9

Yuan, S., Stratton, C. J., Bao, J., Zheng, H., Bhetwal, B. P., Yanagimachi, R. \& Yan, W. (2015). Spata6 is required for normal assembly of the sperm connecting piece and tight head-tail conjunction. Proceedings of the National Academy of Sciences, 112(5), E430-E439. https://doi.org/10.1073/pnas.1424648112

Zarsky, H. A., Cheng, M. \& van der Hoorn, F. A. (2003). Novel RING Finger Protein OIP1 Binds to Conserved Amino Acid Repeats in Sperm Tail Protein ODF1. Biology of Reproduction, 68(2), 543-552. https://doi.org/10.1095/biolreprod.102.009076

Zegers-Hochschild, F., Adamson, G. D., de Mouzon, J., Ishihara, O., Mansour, R., Nygren, K., Sullivan, E. \& Vanderpoel, S. (2009). International Committee for Monitoring Assisted Reproductive Technology (ICMART) and the World Health Organization (WHO) revised glossary of ART terminology, 2009. Fertility and Sterility, 92(5), 1520-1524. https://doi.org/10.1016/j.fertnstert.2009.09.009 
Zeng, X., Li, K., Yuan, R., Gao, H., Luo, J., Liu, F., Wu, Y., Wu, G. \& Yan, X. (2018). Nuclear Envelope-Associated Chromosome Dynamics during Meiotic Prophase I. Frontiers in Cell and Developmental Biology, 5, 121. https://doi.org/10.3389/fcell.2017.00121

Zhen, Y. Y., Libotte, T., Munck, M., Noegel, A. A. \& Korenbaum, E. (2002). NUANCE, a giant protein connecting the nucleus and actin cytoskeleton. Journal of Cell Science, 115(15), 3207-3222. https://jcs.biologists.org/content/115/15/3207

Zhou, J., Du, Y. R., Qin, W. H., Hu, Y. G., Huang, Y. N., Bao, L., Han, D., Mansouri, A. \& Xu, G. L. (2009). RIM-BP3 is a manchette-associated protein essential for spermiogenesis. Development, 136(3), 373-382. https://doi.org/10.1242/dev.030858

Zhu, F., Liu, C., Wang, F., Yang, X., Zhang, J., Wu, H., Zhang, Z., He, X., Zhang, Z., Zhou, P., Wei, Z., Shang, Y., Wang, L., Zhang, R., Ouyang, Y.-C., Sun, Q.-Y., Cao, Y. \& Li, W. (2018). Mutations in PMFBP1 Cause Acephalic Spermatozoa Syndrome. The American Journal of Human Genetics, 103(2), 188-199. https://doi.org/10.1016/j.ajhg.2018.06.010

Zhu, F., Wang, F., Yang, X., Zhang, J., Wu, H., Zhang, Z., Zhang, Z., He, X., Zhou, P., Wei, Z., Gecz, J. \& Cao, Y. (2016). Biallelic SUN5 Mutations Cause Autosomal-Recessive Acephalic Spermatozoa Syndrome. The American Journal of Human Genetics, 99(4), 942-949. https://doi.org/10.1016/j.ajhg.2016.08.004

Zimmerman, W., Sparks, C. A. \& Doxsey, S. J. (1999). Amorphous no longer: The centrosome comes into focus. Current Opinion in Cell Biology, 11(1), 122-128. https://doi.org/10.1016/S0955-0674(99)80015-5 


\section{Appendix}

\subsection{List of oligonucleotides}

Table 6.1.1 Oligonucleotides for mutation screening in patients with acephalic spermatozoon phenotype

\begin{tabular}{|c|c|c|c|c|}
\hline $\begin{array}{c}\text { Target } \\
\text { gene }\end{array}$ & Primer & Sequence & $\begin{array}{c}\text { Annealing } \\
\text { temperature } \\
\left({ }^{\circ} \mathrm{C}\right)\end{array}$ & $\begin{array}{l}\text { Product } \\
\text { size } \\
\text { (bp) }\end{array}$ \\
\hline $\begin{array}{c}\text { Odf1 } \\
\text { Exon1 }\end{array}$ & $\begin{array}{l}\text { Odf1-Homo-Exon1- } \\
\text { Forward } \\
\text { Odf1-Homo-Exon1- } \\
\text { Reverse }\end{array}$ & $\begin{array}{l}\text { TGAGGTCATAGAACACAAGC } \\
\text { CAGGGTCATTATCCTGAACC }\end{array}$ & $54^{\circ} \mathrm{C}$ & 590 bp \\
\hline $\begin{array}{l}\text { Odf1 } \\
\text { Exon2 }\end{array}$ & $\begin{array}{l}\text { Odf1-Homo-Exon2- } \\
\text { Forward } \\
\text { Odf1-Homo-Exon2- } \\
\text { Reverse }\end{array}$ & $\begin{array}{l}\text { GATTCTGAGGTCTGAGCTCCC } \\
\text { GACTTTCACACAACACCAGCAG }\end{array}$ & $54^{\circ} \mathrm{C}$ & 635 bp \\
\hline $\begin{array}{l}\text { Hook1 } \\
\text { Exon } 10\end{array}$ & $\begin{array}{l}\text { Hook1-E10-N-for } \\
\text { Exon10-Rev }\end{array}$ & $\begin{array}{l}\text { CAAGGCTTGAAGCTGCAAAAG } \\
\text { СTTGACСTCССTGTACСTGTC }\end{array}$ & $54^{\circ} \mathrm{C}$ & 453 bp \\
\hline $\begin{array}{l}\text { Hook1 } \\
\text { Exon11 }\end{array}$ & $\begin{array}{l}\text { Hook1-E11-N-for } \\
\text { Hook1-E11-N-Rev }\end{array}$ & $\begin{array}{c}\text { CСТCTTCTCACTCACTCAC } \\
\text { GCTTATTACATGCAACGCTTC }\end{array}$ & $52^{\circ} \mathrm{C}$ & 448 bp \\
\hline $\begin{array}{l}\text { Sun5 } \\
\text { Exon6 }\end{array}$ & $\begin{array}{l}\text { Sun5-Ex7-For } \\
\text { Sun5-Ex7-Rev }\end{array}$ & $\begin{array}{l}\text { CAATGCTGGCAAGTGGCATAG } \\
\text { TGACTGGTTAGGGTGAGACCT }\end{array}$ & $54^{\circ} \mathrm{C}$ & $218 b p$ \\
\hline $\begin{array}{l}\text { Sun5 } \\
\text { Exon8 }\end{array}$ & $\begin{array}{l}\text { Sun5-Ex8-Zhu-For } \\
\text { Sun5-Ex8-Zhu-Rev }\end{array}$ & $\begin{array}{l}\text { ATGAATGGGTCCAGGGATGG } \\
\text { AGATGTTTGGGGCAGAATGG }\end{array}$ & $53^{\circ} \mathrm{C}$ & $284 \mathrm{pb}$ \\
\hline $\begin{array}{l}\text { Sun5 } \\
\text { Exon } 11\end{array}$ & $\begin{array}{l}\text { Sun5-E11-Zhu-For } \\
\text { Sun5-E11-Zhu-Rev }\end{array}$ & $\begin{array}{l}\text { AGGGGATCAAAGGTGGGATG } \\
\text { ACTTCCAGCCTTAAACCAAGC }\end{array}$ & $53^{\circ} \mathrm{C}$ & $376 b p$ \\
\hline $\begin{array}{l}\text { Sun5 } \\
\text { Exon13 }\end{array}$ & $\begin{array}{l}\text { Sun5-E13-Zhu-For } \\
\text { Sun5-E13-Zhu-Rev }\end{array}$ & $\begin{array}{c}\text { TGTCTGTCCTTCTGGCCTC } \\
\text { TCAGATGTGAAAGGCTAGCC }\end{array}$ & $52^{\circ} \mathrm{C}$ & 437bp \\
\hline $\begin{array}{l}\text { Arl3 } \\
\text { Exon3 }\end{array}$ & $\begin{array}{l}\text { Arl3-E3-For } \\
\text { Arl3-E3-Rev }\end{array}$ & $\begin{array}{l}\text { CCACAACCTGGATGAAACAAGCA } \\
\text { CAGTGGCATGATCTTCTGACTTCC }\end{array}$ & $55^{\circ} \mathrm{C}$ & $613 b p$ \\
\hline
\end{tabular}


Table 6.1.2 Oligonucleotides for RT-PCR in Spag4-deficient mouse

\begin{tabular}{|c|c|c|c|c|}
\hline $\begin{array}{l}\text { Target } \\
\text { gene }\end{array}$ & Primer & Sequence & $\begin{array}{c}\text { Annealing } \\
\text { temperature } \\
\left({ }^{\circ} \mathrm{C}\right)\end{array}$ & $\begin{array}{c}\text { Product } \\
\text { size } \\
\text { (bp) }\end{array}$ \\
\hline \multirow[t]{2}{*}{ Gapdh } & rGAPDH-For3 & GTCAGATCCACGACGGACAC & \multirow[t]{2}{*}{$52^{\circ} \mathrm{C}$} & \multirow[t]{2}{*}{$595 \mathrm{bp}$} \\
\hline & rGAPDH-Rev2 & GTATGACTCCACTCACGGCA & & \\
\hline \multirow[t]{2}{*}{ Spag4 } & Spag4-650for & TGCAGGGAAATCTGCTCC & \multirow[t]{2}{*}{$55^{\circ} \mathrm{C}$} & \multirow[t]{2}{*}{$546 \mathrm{bp}$} \\
\hline & Spag4C2-KpnI & GGTACCTTAGAGTCAGCATCTCCGTAGGTTC & & \\
\hline \multirow[t]{2}{*}{ Stra8 } & Stra8 For & TCACAGCCTCAAAGTGGCAGG & \multirow[t]{2}{*}{$56^{\circ} \mathrm{C}$} & \multirow[t]{2}{*}{$444 \mathrm{bp}$} \\
\hline & Stra8 Rev & GCAACAGAGTGGAGGAGGAGT & & \\
\hline \multirow[t]{2}{*}{ Sycpl } & MmSycp1f & CGCTACAACCACATGCTTCG & \multirow[t]{2}{*}{$52^{\circ} \mathrm{C}$} & \multirow[t]{2}{*}{$200 \mathrm{bp}$} \\
\hline & MmSycp1f & GGAACGCTGGTTAGATCTCCTC & & \\
\hline \multirow[t]{2}{*}{ Sycp3 } & MSycp3-5 KpnI & GACGGTACCATGCTTCGAGGGTGTGGG & \multirow[t]{2}{*}{$52^{\circ} \mathrm{C}$} & \multirow[t]{2}{*}{$900 \mathrm{bp}$} \\
\hline & MSycp3r & TTGACACAATCGTGGAGAGAA & & \\
\hline \multirow[t]{2}{*}{ Odfl } & Odf1C & CCGCGGTACCCAAGATCATCTTCCTACA & \multirow[t]{2}{*}{$54^{\circ} \mathrm{C}$} & \multirow[t]{2}{*}{$800 \mathrm{bp}$} \\
\hline & Odf1N-TomHindIII & AAGCTTATGGCCGCACTGAGTTG & & \\
\hline \multirow[t]{2}{*}{ Odf1 } & Odf1N434 & CCTCTACTACCCGTGCTG & \multirow[t]{2}{*}{$52^{\circ} \mathrm{C}$} & \multirow[t]{2}{*}{$548 \mathrm{~b}$} \\
\hline & Odf1C & CCGCGGTACCCAAGATCATCTTCCTACA & & \\
\hline \multirow[t]{2}{*}{ Prml } & mProtamin $1 \mathrm{f}$ & TCCACCTGCTCACAGGTTGGC & \multirow[t]{2}{*}{$52^{\circ} \mathrm{C}$} & \multirow[t]{2}{*}{$407 \mathrm{bp}$} \\
\hline & mProtamin $1 \mathrm{r}$ & GCAGGCTCCTGTTTTTCATCG & & \\
\hline \multirow[t]{2}{*}{ Hanpl } & Hanp1-HIT2-for & GCTGGCTACTTCAGGGTCT & \multirow[t]{2}{*}{$50^{\circ} \mathrm{C}$} & \multirow[t]{2}{*}{800 bp } \\
\hline & Hanp1-HIT2-rev & TGTATGCTGGGAGCGTTG & & \\
\hline \multirow[t]{2}{*}{$\alpha$-Tubulin } & Ma3_For & TTGAGGACCAGTGGTGAGG & \multirow[t]{2}{*}{$55^{\circ} \mathrm{C}$} & \multirow[t]{2}{*}{$1464 \mathrm{bp}$} \\
\hline & Ma3_Rev & TTTCCAATGGTGGGGAAGACAT & & \\
\hline \multirow[t]{2}{*}{$\alpha$-Tubulin } & Ma7_For & CTCGTAGGATCGGCTGAGGTA & \multirow[t]{2}{*}{$54^{\circ} \mathrm{C}$} & \multirow[t]{2}{*}{$1400 \mathrm{bp}$} \\
\hline & Ma7_Rev & CCATGCGCTCAGTACTCCT & & \\
\hline
\end{tabular}


Table 6.1.3 Oligonucleotides for qRT-PCR in Spag4-deficient mouse

\begin{tabular}{llc}
\hline \multicolumn{1}{c}{ Target gene } & \multicolumn{1}{c}{ Primer } & \multicolumn{1}{c}{ Sequence } \\
HPRT & mHPRT-For2 & GGAGTCCTGTTGATGTTGCC \\
\cline { 2 - 3 } & mHPRT-Rev2 & GGGACGCAGCAACTGACATT \\
\hline Protamin & Protamin-p1-For & CCCTCTCACCACTTTTCTTACCTT \\
\cline { 2 - 3 } & Protamin-p1-For & GGCGAGATGCTCTTGAAGTCT \\
\hline Stra8 & Stra8 For & TCACAGCCTCAAAGTGGCAGG \\
\cline { 2 - 3 } Odf1 & Stra8 Rev & GCAACAGAGTGGAGGAGGAGT \\
& ODF1-p1-Forward & CGGGGCTCTTTTAGTCTTGCTA \\
\cline { 2 - 3 } Hanp1 & ODF1-p1-Reverse & TTCAAAGCCGCACACATTCAC \\
\hline & qHANP1-For & CTGCTGGCTACTTCAGGGTCTGG \\
\hline
\end{tabular}

Table 6.1.4 Oligonucleotides for amber suppression method

\begin{tabular}{|c|c|c|c|c|}
\hline $\begin{array}{l}\text { Target } \\
\text { gene }\end{array}$ & Primer & Sequence & $\begin{array}{l}\text { Annealing } \\
\text { temperature } \\
\left({ }^{\circ} \mathbf{C}\right)\end{array}$ & $\begin{array}{l}\text { Product } \\
\text { size (bp) }\end{array}$ \\
\hline Sun1 & $\begin{array}{c}\text { Sun1-NheI-F } \\
\text { Sun1-BamHI-Rev2 }\end{array}$ & $\begin{array}{c}\text { 5'GGTCTGAGACGGTGCTAGCAACATGGACTTT } \\
\text { TCTC-3' } \\
\text { CTAGGATCCTGGATGGGCTCTCCG }\end{array}$ & $62^{\circ} \mathrm{C}$ & $2200 \mathrm{bp}$ \\
\hline Sun3 & $\begin{array}{l}\text { Sun3-NheI-For } \\
\text { Sun3-KpnI-Rev }\end{array}$ & $\begin{array}{l}\text { CGCTAGCAATGTTAACTCGATCATGGAAG } \\
\text { GGGTACCGCAGTGTAATCACTGGGGATG }\end{array}$ & $65^{\circ} \mathrm{C}$ & 975 bp \\
\hline Lamin C2 & $\begin{array}{l}\text { LmnC2-XbaI-For } \\
\text { LmnC2-KpnI-Rev }\end{array}$ & $\begin{array}{l}\text { TCTAGAGGCCCAGCCATGGGGAAC } \\
\text { CGGTACCAGCGGCGGCTGCCACTCAC }\end{array}$ & $58^{\circ} \mathrm{C}$ & $1408 \mathrm{bp}$ \\
\hline Lamin B3 & $\begin{array}{l}\text { LamnB3-XbaI-For } \\
\text { LmnB3-KpnI-Rev }\end{array}$ & $\begin{array}{c}\text { CTCTAGAGCCATGGGGGAGTCGGAATC } \\
\text { TGGTACCATCAGTCGGCAGCCCCTTG }\end{array}$ & $58^{\circ} \mathrm{C}$ & $1438 \mathrm{bp}$ \\
\hline
\end{tabular}


Table 6.1.5 Oligonucleotides for rapamycin system method

\begin{tabular}{|c|c|c|c|c|}
\hline $\begin{array}{c}\text { Target } \\
\text { gene }\end{array}$ & Primer & Sequence & $\begin{array}{c}\text { Annealing } \\
\text { temperature } \\
\left({ }^{\circ} \mathbf{C}\right)\end{array}$ & $\begin{array}{c}\text { Product } \\
\text { size (bp) }\end{array}$ \\
\hline $\begin{array}{c}m E C F P- \\
F R B\end{array}$ & $\begin{array}{c}\text { ECFP-N-NheI } \\
\text { FIASH-C-NheI }\end{array}$ & $\begin{array}{c}\text { GCTAGCATGGGCTGCGTGTGCAGCAG } \\
\text { GCTAGCGGGCTCCATGCAGCAGCCG }\end{array}$ & $58^{\circ} \mathrm{C}$ & $1100 \mathrm{bp}$ \\
\hline $\begin{array}{c}\text { Luciferase } \\
(p G L 3)\end{array}$ & $\begin{array}{c}\text { Luc-NheI-For2 } \\
\text { Luc-AgeI-Rev2 }\end{array}$ & $\begin{array}{c}\text { GAGCTAGCATGGAAGACGCCAAAAACAT } \\
\text { TAACCGGTGCCACGGCGATCTTTCCGCC }\end{array}$ & $58^{\circ} \mathrm{C}$ & $1668 \mathrm{bp}$ \\
\hline Sun5 & $\begin{array}{c}\text { Sun-SacI-N-Rap } \\
\text { Sun5-KpnI-C-Rap }\end{array}$ & GAGCTCATGCCCCGGACGAGGAAC & $60^{\circ} \mathrm{C}$ & $1130 \mathrm{bp}$ \\
\hline
\end{tabular}

Table 6.1.6 Oligonucleotides for Spag4l2 investigation

\begin{tabular}{|c|c|}
\hline Oligonucleotide name & Oligonucleotides sequence \\
\hline Primer1 & CGGCTAGCGCCACCATGGCCTACCCCTACGACGTG \\
\hline Primer2 & GCCTACCCCTACGACGTGCCCGACTACGCCTCTAGA \\
\hline primer 3 TM2 & CCCGACTACGCCTCTAGAATGAAGATGGGCCTTCTGGTCCTC \\
\hline TM38-58r & GGAATTCGCTGGGTCTGAGTCCTCAGGAA \\
\hline Spag77-95r EcoRI & GGAATTCGTAAGTGCATGGAAAACATCCA \\
\hline Spag4XbaI for & GCTCTAGAGCAATGCCCCGGACGAGGAACATC \\
\hline Spag4KpnI-frameshift & AGCTTGGTACCGACTGGCAAAGGCCCAGCAGTTC \\
\hline Spag4lC-term-F-HindIII & CCAAGCTTATGCCATCAAAAGTGGAAGTCTGG \\
\hline Spag4l C-term-rev-EcoRI & GGAATTCGGGATAGGGGCTCTAGGTGTGA \\
\hline
\end{tabular}


Table 6.1.7 Oligonucleotides for Spag4L2-deficient mice genotyping.

\begin{tabular}{|c|c|c|c|c|}
\hline Target gene & Primer & Sequence & $\begin{array}{c}\text { Annealing } \\
\text { temperature } \\
\left({ }^{\circ} \mathrm{C}\right)\end{array}$ & $\begin{array}{l}\text { Product } \\
\text { size (bp) }\end{array}$ \\
\hline \multirow[t]{2}{*}{ LacZ } & LacInZRev & GTCTGTCCTAGCTTCCTCACTG & $54^{\circ} \mathrm{C}$ & $694 \mathrm{bp}$ \\
\hline & SunUp & TGGCATCCTCTGAGGGAG & & \\
\hline \multirow[t]{2}{*}{ LacZ } & LacInFor & GGTAAACTGGCTCGGATTAGGG & $55^{\circ} \mathrm{C}$ & $211 \mathrm{bp}$ \\
\hline & LacInRev & TTGACTGTAGCGGCTGATGTTG & & \\
\hline \multirow[t]{2}{*}{ Neomycin } & NeoInFor & TTCGGCTATGACTGGGCACAACAG & $55^{\circ} \mathrm{C}$ & $282 \mathrm{bp}$ \\
\hline & NeoInRev & TACTTTCTCGGCAGGAGCAAGGT & & \\
\hline \multirow[t]{2}{*}{ Neomycin } & NeoFwd & TCATTCTCAGTATTGTTTTGCC & $52^{\circ} \mathrm{C}$ & $507 \mathrm{bp}$ \\
\hline & SunDown & GAGACTGAGGCCCTAGGAGG & & \\
\hline \multirow[t]{2}{*}{ Neomycin } & NeomycinFor & GAACAAGATGGATTGCACG & $50^{\circ} \mathrm{C}$ & $1239 \mathrm{bp}$ \\
\hline & NeoSplitRev & GCAAAACAATACTGAGAATG & & \\
\hline \multirow[t]{2}{*}{ Neomycin } & NeomycinFor & GAACAAGATGGATTGCACG & $48^{\circ} \mathrm{C}$ & $333 \mathrm{bp}$ \\
\hline & NeoInRev & TACTTTCTCGGCAGGAGCAAGGT & & \\
\hline \multirow[t]{2}{*}{ Neomycin/Sun5 3'end } & NeomycinFor3 & GTCAAGACCGACCTGTCCGG & $56^{\circ} \mathrm{C}$ & $1441 \mathrm{bp}$ \\
\hline & Genotype2Rev & GAGCGGTCACTGTCCTCCTATG & & \\
\hline \multirow{2}{*}{$\begin{array}{l}\text { Neomycin and Sun5 } \\
\text { 3'end }\end{array}$} & Geno2For & AGGACATAGCGTTGGCTACC & $52^{\circ} \mathrm{C}$ & $1109 \mathrm{bp}$ \\
\hline & Geno2Rev & TAGCTGTCATCCTGCCCCTA & & \\
\hline \multirow{2}{*}{$\begin{array}{l}\text { Sun5 } \\
\text { wild type allele }\end{array}$} & GenotypeFor2 & GGAGCTGCTACACTCTGGCTC & $57^{\circ} \mathrm{C}$ & $447 \mathrm{bp}$ \\
\hline & GenotypeRev2 & GAGCGGTCACTGTCCTCCTATG & & \\
\hline
\end{tabular}

Table 6.1.8 Oligonucleotides for qPCR for Spag4l2-deficient mice and ES cells.

\begin{tabular}{llc}
\multicolumn{1}{c}{ Target } & \multicolumn{1}{c}{ Primer } & \multicolumn{1}{c}{ Sequence } \\
\hline Protamin & Protamin-p1-For & CCCTCTCCACCACTTTTCTTACCTT \\
\cline { 2 - 3 } Odf1 & Protamin-p1-Rev & GGCGAGATGCTCTTGAAGTCT \\
\cline { 2 - 3 } Exon7 & ODF1-p1-Forward & CGGGGCTCTTTTAGTCTTGCTA \\
\cline { 2 - 3 } & ODF1-p1-Reverse & TTCAAAGCCGCACACATTCAC \\
& Sun5-mus-E5-For & GGTAGACCCAGGAGAGGGAA \\
\cline { 2 - 3 } Exon 9 & Sun5-mus-E5-Rev & TGGTAAAGGGTCTAACGGCA \\
& Sun5-mus-E8-For & GGGTGCCCTCAGAACTTGTC \\
\cline { 2 - 3 } & Sun5-mus-E8-Rev & CCTCTTCTGGCCACCTCCTA \\
\hline
\end{tabular}




\section{2 List of abbreviations}

${ }^{\circ} \mathrm{C}$

ASS

AnP

APS

Arl3

ART

$\mathrm{Bm}$

bp

BSA

CCDC42

cDNA

CFAP52/WDRPUH/WDR16

Cntrob

$\mathrm{CO}_{2}$

Co-IP

$\mathrm{COOH}$-terminus/C-terminus

$\mathrm{Ct}$

C-X-P

DAPI

$\mathrm{ddH}_{2} \mathrm{O}$

DEPC

DH10b

DH5 $\alpha$

DMEM

DMSO

DNA

Dnase

dsDNA

DTT

ECFP

ECL

EDTA

ER

ES

et al.

FBS

Fig

FKBP

FRB

$\mathrm{Fu}$

G1-S
Degree centigrade

Acephalic spermatozoa syndrome

Antarctic Phosphatase

Ammonium persulfate

ADP-ribosylation factor-like protein 3

Assisted reproductive technology

Mitosis of spermatogonia

Base pair(s)

Bovine serum albumin

Coiled-coil domain containing 42

complementary DNA

Cilia and flagella associated protein 52

Centrobin

Carbon dioxide

Co-immunoprecipitation

Carboxyl-terminal

Cycle threshold

Cysteine-X-proline

4,6-diamidino-2-phenylindole

ultra-pure and sterile water

Diethyl pyrocarbonate

10-beta competent E.coli

5-alpha Competent E.coli

Dulbecco's Modified Eagle's Medium

Dimethyl sulfoxide

Deoxyribonucleic acid

Deoxyribonuclease

double-stranded desoxyribonucleic acid

Dithiothreitol

enhanced cyan fluorescent protein

enhanced chemiluminescence

Ethylenediaminetetraacetic acid

Endoplasmic reticulum

Embryonic stem

et alii (and others)

Fetal bovine serum

Figure

FK506 binding protein

FKBP rapamycin binding

Protein serine-threonine kinase Fused

Transition phase 
GAPDH

gDNA

HA

HANP1/ HIT2

HEK293

HEPES

HOOK1

HPRT

HPRT

HRP

HSPB 10

HTCA

ICSI

Ift 88

$\mathrm{IgG}$

${ }^{\text {In }} \mathrm{m}$

INM

IPTG

$\mathrm{KASH}$

$\mathrm{kb}$

$\mathrm{kDa}$

KIF27

KLC3

Ko

KOAc

LB

LINC

LMNA

LMNB 1

LMNB2

LMNC

LoxP

LSM

$\mathrm{M}$

$\mathrm{m} 2^{\circ} \mathrm{m}$

MBP

$\mathrm{mg}$

$\mathrm{m}^{\mathrm{In}}$

$\mathrm{mL}$

MTOC

MYL6

$\mathrm{M} \alpha 3$

$\mathrm{M} \alpha 7$

NH2-terminal/N-terminal
Glyceraldehyde-3-Phosphate Dehydrogenase Genomic deoxyribonucleic acid Human influenza hemagglutinin Human testis-specific $\mathrm{H} 1$ histone Human embryonic kidney cell line Hydroxyethyl piperazineethanesulfonic acid Protein Hook homolog 1

Hypoxanthine phosphoribosyltransferase Hypoxanthine phosphoribosyltransferase Horseradish peroxidase Heat shock protein B10 Head-to-tail coupling apparatus Intracytoplasmic sperm injection Intraflagellar Transport 88

Immunoglobulin $\mathrm{G}$

Intermediate spermatogonia

Inner nuclear membrane

isopropil- $\beta$-D-1-tiogalactopiranósido

Klarsicht/ANC-1/Syne-1 homology

kilobase

kilodalton

Kinesin protein

Kinesin light chain 3

Knock out

Potassium acetate

Lysogeny broth

Linker of nucleoskeleton and cytoskeleton lamin A

lamin B1

lamin B2

lamin $\mathrm{C}$

locus of X-over P1

Confocal laser scanning microscopy

molar

Second meiotic division

maltose-binding protein

Milligram(s)

Mitosis of intermediate spermatogonia

Millimeter(s)

Microtubule-organizing center

Myosin

mouse $\alpha 3$-tubulin

mouse $\alpha 7$-tubulin

Amino-terminal 
NIH3T3

Oaz3

OD

ODF1

ODF2

ODFs

ONM

PAGE

$p$ Bpa

PBS

PCM

PCR

PFA

PK

$\mathrm{Pl}$

PL-FITC

PMC

PMFBP1

PMSF

Prm1

Prss21

PS

qPCR

qRT-PCR

RIM-BP3

RNA

rRNA

RS

RT-PCR

S.O.C

SDS-PAGE

sHSPs

SPAG4/SUN4

SPAG4L/SUN5

SPAG4L2

SPATA6

Stk36

Stra8

SUN

SYCP1

SYCP3

SYNE-1

TAE

TBST

TEMED
Mouse embryonic fibroblast

Ornithine Decarboxylase Antizyme 3

optical density

Outer Dense Fiber of Sperm Tails 1

Outer Dense Fiber of Sperm Tails 2

Outer dense fibers

Outer nuclear membrane

Polyacrylamide gel electrophoresis

Unnatural amino acid p-benzoyl-L-phenylalanine

Phosphate-buffered saline

Pericentriolar material

Polymerase chain reaction

Paraformaldehyde

Protein kinase

Preleptotene

Fluorescein isothiocyanate-labeled peanut lectin

Dense pericentriolar matrix

Polyamine modulated factor 1 binding protein 1

Phenylmethanesulfonyl fluoride

Protamine 1

Glycosylphosphatidylinositol-Anchored Serine Protease

Perinuclear space

Real-time PCR

Real-Time Quantitative Reverse Transcription PCR

RIMS Binding Protein 3

Ribonucleic acid

Ribosomal ribonucleic acid

Round spermatids

reverse transcription-PCR

Super Optimal broth with Catabolite repression

Sodium dodecyl sulfate-polyacrylamide gel electrophoresis

Small heat shock proteins

Sperm associated antigen 4

Sad1 and UNC84 domain containing 5-like

Sad1 and UNC84 domain containing 5

Spermatogenesis Associated 6

Serine/Threonine Kinase 36

Stimulated by retinoic acid gene 8

Sad1p, UNC-84 domains

Synaptonemal complex protein 1

Synaptonemal complex protein 3

Synaptic nuclear envelope protein 1

Tris-acetate-EDTA

Tris-buffered saline with Tween 20

Tetramethylethylenediamine 
TM1

TM2

TMs

tRNA

tRNA-aaRS

TZI

$\mathrm{U}$

UAAs

UV

$\mathrm{V}$

$\mathrm{v} / \mathrm{v}$

$\mathrm{w} / \mathrm{v}$

WD40

WHO

WT

$\mathrm{x} g$ (or RFC)

$\mu \mathrm{g}$

$\mu 1$

$\mu \mathrm{M}$

$\mu \mathrm{m}$
Transmembrane domain I

Transmembrane domain II

Transmembrane domains

Transfer ribonucleic acid

Orthogonal aminoacyl tRNA synthetase-tRNA

Human teratozoospermia

Units

Unnatural amino acids

Ultraviolet

Volt

volume/volume

weight/volume

WD40 domain-containing proteins

World Health Organization

Wild type

relative centrifugal force

microgram(s)

microliter(s)

micromolar

micrometer(s) 